\title{
Interaction of antimicrobial compounds with lipid bilayers in established and novel membrane model systems
}

\author{
Dissertation \\ for the award of the degree \\ Doctor rerum naturalium \\ of the Georg-August University Göttingen \\ within the doctoral program \\ Chemistry of the Georg-August University School of Science
} (GAUSS)

submitted by

\section{Johannes Schumacher \\ from Nürnberg}

Göttingen 2020 
Members of the Thesis Committee:

Prof. Dr. Claudia Steinem

Institute of Organic and Biomolecular Chemistry

Georg-August University Göttingen

Prof. Dr. Michael Meinecke

Dept. of Cellular Biochemistry

University Medical Center Göttingen

Members of the Examination Board:

Prof. Dr. Claudia Steinem (Reviewer)

Institute of Organic and Biomolecular Chemistry

Georg-August University Göttingen

Prof. Dr. Michael Meinecke (Second Reviewer)

Dept. of Cellular Biochemistry

University Medical Center Göttingen

Further members of the Examination Board:

Prof. Dr. Helmut Grubmüller

Dept. Theoretical and Computational Biophysics

Max Planck Institute for Biophysical Chemistry

Dr. Sebastian Kruss

Institute for Physical Chemistry

Georg August University Göttingen

Prof. Dr. Marcus Müller

Institute for Theoretical Physics

Georg-August University Göttingen

Prof. Dr. Silvio O. Rizzoli

Dept. of Neuro- and Sensory Physiology

University Medical Center Göttingen

Date of oral examination:

06.05.2020 
"I may not have gone where I intended to go, but I think I have ended up where I needed to be."

- Douglas Adams, The Long Dark Tea-Time of the Soul 


\section{Declaration}

I, Johannes Schumacher, hereby certify, that this doctoral thesis entitled "Interaction of antimicrobial compounds with lipid bilayers in established and novel membrane model systems" is my own work. It was written independently and with no other sources and aids than those specified in the text.

Göttingen, 2020

Johannes Schumacher 


\section{Abstract}

Investigating the interaction between antimicrobial compounds and membrane model systems in the form of artificial lipid bilayers provides important insights into their mode of action.

The interaction of lipid side chains (LSCs) of the nucleoside antibiotic muraymycin with membranes was characterized by measuring the influx of fluorescently labeled LSCs into adhered giant unilamellar vesicles (GUVs) via confocal laser scanning microscopy (CLSM). No bilayer destabilization was detected for POPC and POPC/POPE 7:3 for any LSC at 0.8 $\mu \mathrm{M}$. Furthermore, the partitioning of the LSCs into the vesicle bilayer was quantified. The partitioning of $\omega$-functionalized tridecanoic acid derivates was found to correlate with the antibacterial potency of the corresponding muraymycins. Increasing PE content in the bilayer led to a decreased accumulation of LSCs. Unfunctionalized tridecanoic acid showed increased partitioning, and no negative influence of PE. This suggests that the antibacterial potency of muraymycin could be improved by exchanging the natural LSCs for long acyl chains.

The mode of action of the antimicrobial cyclic peptide lugdunin was investigated with several bulk vesicle assays via fluorescence spectroscopy. The unspecific permeabilization of lipid bilayers composed of POPC and POPC/POPG 1:1 by lugdunin was shown to be negligible in a carboxyfluorescein dequenching assay. An indication for lugdunin-mediated $\mathrm{Na}^{+}$and $\mathrm{K}^{+}$ transport was found in vesicles acidified via a potassium gradient. Follow-up experiments were conducted in vesicles containing the $\mathrm{pH}$-sensitive fluorescent dye pyranine and a direct proton gradient was established via $\mathrm{pH}$-change around the vesicles. Lugdunin was shown to transport protons, $\mathrm{Na}^{+}, \mathrm{K}^{+}$, and to a lesser degree $\mathrm{Cs}^{+}$. Variations of the assay confirmed that the rate limiting process was peptide insertion rather than ion transport. Negative lipid headgroups had no significant effect on the insertion rate, while an influence of the peptide stock-solution solvent was observed, suggesting that a conformational change in the peptide could limit insertion. The reaction order in reference to lugdunin was found to be higher than one, indicating an oligomerization of the peptide, e.g. by stacking in the bilayer to form pores.

An established membrane permeabilization assay for the characterization of antimicrobial peptides, based on pore-spanning lipid bilayers spread on optically transparent porous alumina (AAO), was further developed. The spreading of phase-separated GUVs on the porous substrate revealed lipid reorganization between solid-supported and freestanding membranes. The assay was modified to allow the application of a transmembrane potential. To this end, an electrode array in the form of silver nanowires was deposited into the porous substrate. The application of a potential to the electrode array caused a transmembrane potential across pore-spanning membranes, which was verified with the voltage-sensitive fluorescence probe $\operatorname{DiOC}_{2}(3)$ via CLSM. Proton transport along the applied potential was shown by ratiometric CLSM of pyranine enclosed in the substrate pores. The interaction of pyranine with $\mathrm{AgCl}$ formed during the chloridation of the electrode array was found to interfere with the quantitative readout of the permeabilization rate. The orthogonal functionalization of the AAO substrate by thermal evaporation of $\mathrm{SiO}$ was demonstrated successfully, which reduced complexity and improved substrate stability. 
Zusammenfassung

Die Untersuchung der Wechselwirkung zwischen antimikrobiellen Verbindungen und MembranModellsystemen in Form von künstlichen Lipid-Doppelschichten liefert wichtige Erkenntnisse über deren Wirkungsweise. Die Wechselwirkung der Lipidseitenketten (LSCs) des NukleosidAntibiotikums Muraymycin mit Membranen wurde durch die Bestimmung des Einstroms fluoreszenzmarkierter LSCs in adhärierte riesige unilamellare Vesikel (GUVs) mittels konfokaler Laser-Scanning-Mikroskopie (CLSM) charakterisiert. Für POPC und POPC/POPE 7:3 wurde bei 0,8 $\mu \mathrm{M}$ für keine der LSCs eine Destabilisierung der Lipiddoppelschicht festgestellt. Darüber hinaus wurde die Partitionierung der LSCs in die Lipiddoppelschicht der Vesikel-quantifiziert. Die Partitionierung von $\omega$-funktionalisierten Tridecansäure-Derivaten korrelierte mit der antibakteriellen Potenz der entsprechenden Muraymycine. Ein zunehmender PE-Gehalt in der Doppelschicht führte $\mathrm{zu}$ einer verringerten Anreicherung von LSCs. Unfunktionalisierte Tridecansäure zeigte eine erhöhte Partitionierung und keinen negativen Einfluss von PE. Dies deutet darauf hin, dass die antibakterielle Wirksamkeit von Muraymycin durch den Austausch der natürlichen Seitenketten gegen lange Acyl-Ketten verbessert werden könnte.

Die Wirkungsweise des antimikrobiellen zyklischen Peptids Lugdunin wurde mit mehreren BulkVesikel-Assays mittels Fluoreszenzspektroskopie untersucht. Die unspezifische Permeabilisierung von Lipiddoppelschichten aus POPC und POPC/POPG 1:1 durch Lugdunin erwies sich in einem Carboxyfluorescein dequenching-Assay als vernachlässigbar. In Vesikeln, die über einen Kaliumgradienten angesäuert wurden, wurden Hinweise auf einen Lugdunin-vermittelten $\mathrm{Na}^{+}-$ und $\mathrm{K}^{+}$-Transport gefunden. Folgeexperimente wurden in Vesikeln durchgeführt, die den $\mathrm{pH}$ empfindlichen Fluoreszenzfarbstoff Pyranin enthielten, und ein direkter Protonengradient wurde über eine pH-Wert-Änderung um die Vesikel herum hergestellt. Es wurde gezeigt, dass Lugdunin Protonen, $\mathrm{Na}^{+}, \mathrm{K}^{+}$und in geringerem Maße auch $\mathrm{Cs}^{+}$transportiert. Variationen des Assays bestätigten, dass der geschwindigkeitsbestimmende Prozess die Peptidinsertion und nicht der Ionentransport war. Negative Lipidkopfgruppen hatten keinen signifikanten Einfluss auf die Insertionsrate, während ein Einfluss des Peptid-Lösungsmittels beobachtet wurde, was darauf hindeutet, dass eine Konformationsänderung des Peptids die Insertion bestimmen könnte. Die Reaktionsordnung in Bezug auf Lugdunin war höher als eins, was auf eine Oligomerisierung des Peptids hinweist, z.B. durch gestapelte Aggregation zu Poren in der Membran.

Ein etablierter Membran-Permeabilisierungsassay zur Charakterisierung antimikrobieller Peptide, basierend auf porenüberspannenden Lipid-Doppelschichten auf optisch transparentem porösen Aluminiumoxid, wurde weiterentwickelt. Die Spreiten von phasenseparierten GUVs auf dem porösen Substrat resultierte in einer Lipidreorganisation zwischen feststoffgestützten und freistehenden Membranen. Der Assay wurde modifiziert, um die Anwendung eines Transmembranpotentials zu ermöglichen. Zu diesem Zweck wurde eine Elektrodenanordnung in Form von Silbernanodrähten in das poröse Substrat eingebracht. Das Anlegen eines Potentials an das Elektrodenarray bewirkte ein Transmembranpotential an porenüberspannende Membranen, das mit der spannungsempfindlichen Fluoreszenzsonde $\operatorname{DiOC}_{2}(3)$ über CLSM nachgewiesen wurde. Der Protonentransport entlang des angelegten Potentials wurde durch ratiometrisches CLSM von in den Substratporen eingeschlossenem Pyranin gezeigt. Die Wechselwirkung von Pyranin mit $\mathrm{AgCl}$, das während der Chloridierung des Elektrodenarrays gebildet wurde, störte die quantitative Bestimmung der Permeabilisierungsrate. Die orthogonale Funktionalisierung des AAO-Substrats durch thermisches Aufdampfen von $\mathrm{SiO}$ wurde erfolgreich demonstriert, wodurch die Komplexität reduziert und die Substratstabilität verbessert wurde. 




\section{Table of Contents}

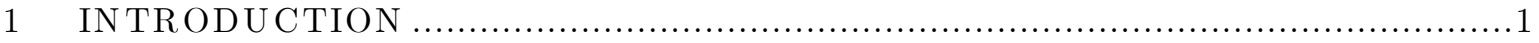

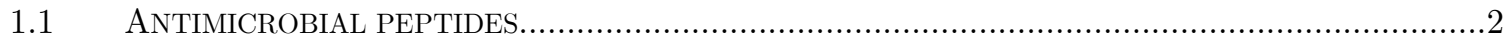

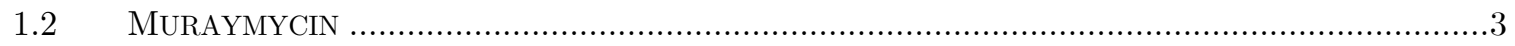

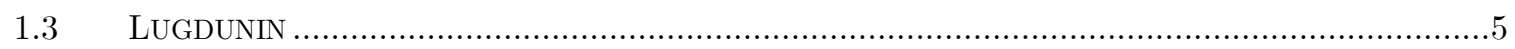

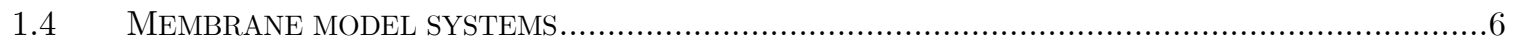

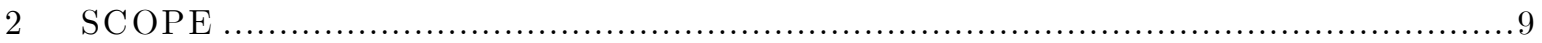

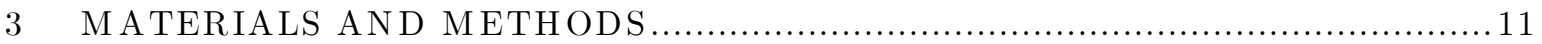

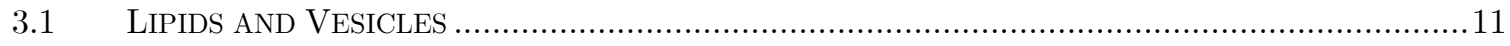

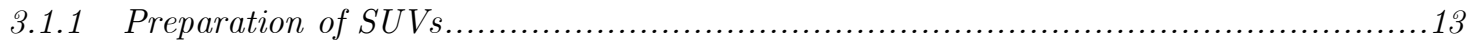

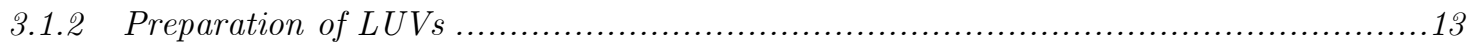

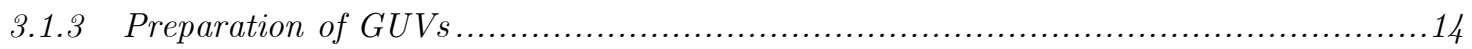

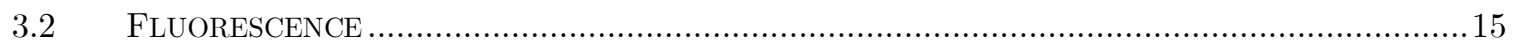

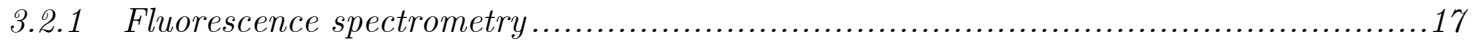

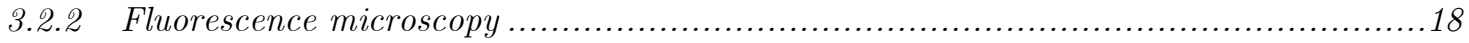

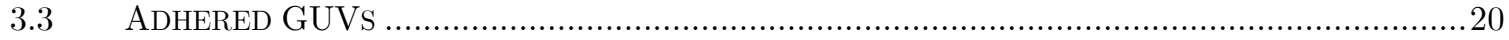

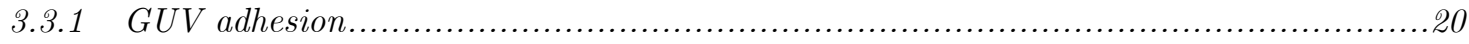

3.3.2 Permeabilization and Partitioning assay ................................................2

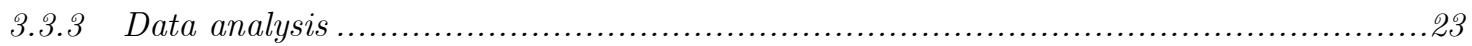

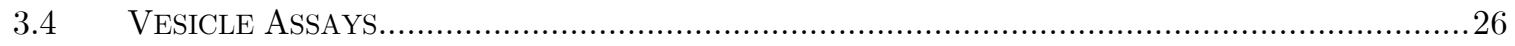

3.4.1 Permeabilization assay with carboxyfluorescein dequenching .............................26

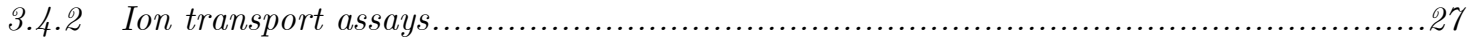

3.5 PORE-SPANNING MEMBRANES ON POROUS ALUMINIUM OXIDE ……...................................31

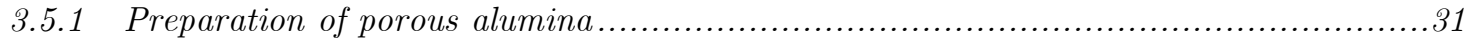

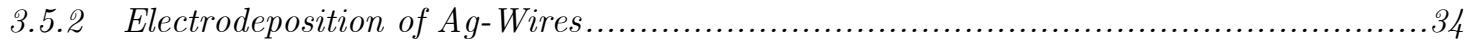

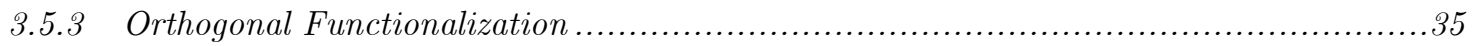

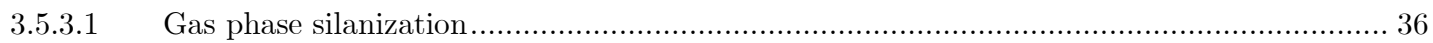

3.5.3.2 Fluid phase silanization and PEGylation ……............................................................... 37

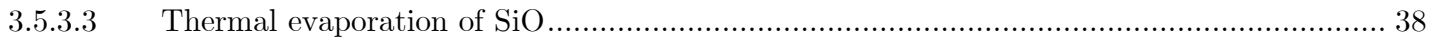

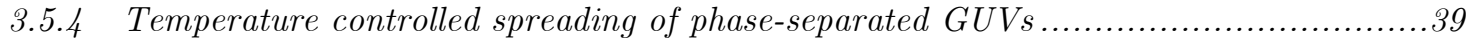

3.5.5 Combination of microscopy and electrochemistry ......................................40 
RESULTS AND DISCUSSION .......................................................... 43

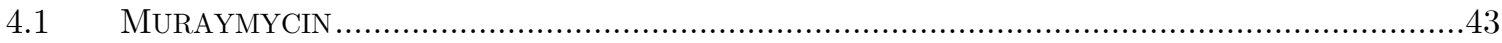

4.1.1 Influx of muraymycin lipid side chains into adhered GUVs ........................... 44

4.1.2 Partitioning of labeled muraymycin lipid side chains into lipid bilayers .................. 51

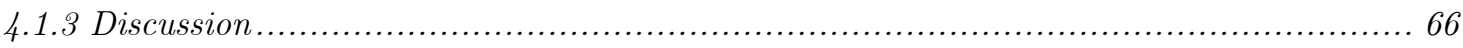

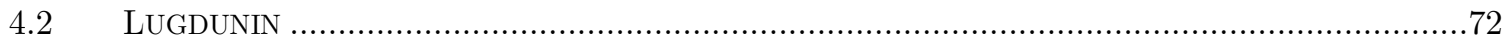

4.2.1 Permeabilization of lipid bilayers by lugdunin .......................................... 72

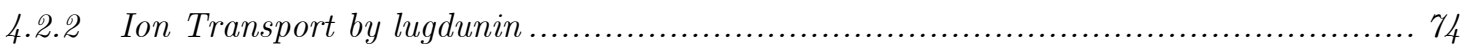

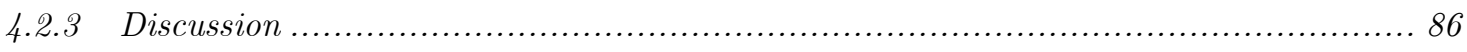

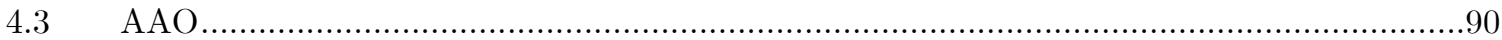

4.3.1 Permeabilization assay on phase-separated lipid bilayers ............................... 91

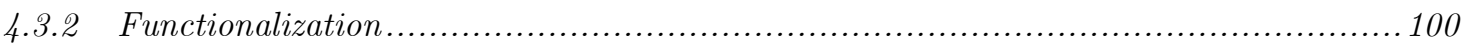

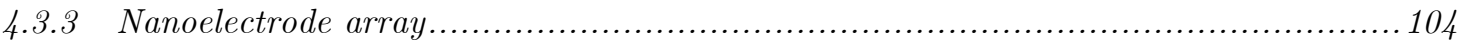

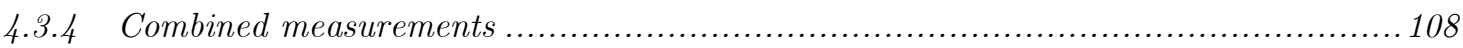

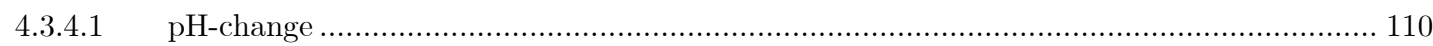

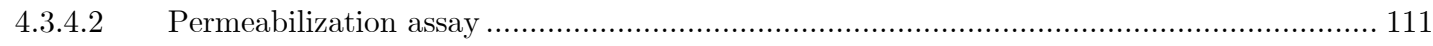

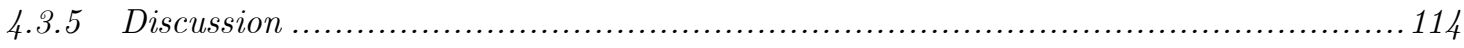

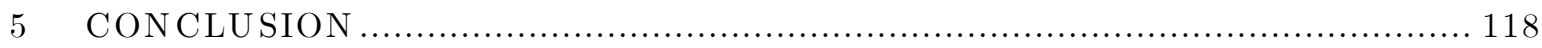

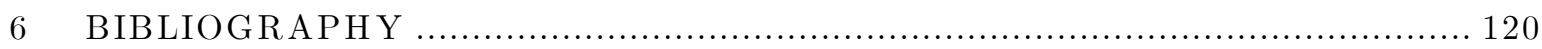

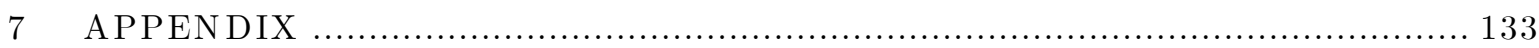




\section{Introduction}

The development of antibiotics in the last century has arguably had the biggest impact on medicine in recent history and has been credited to be the main reason for the steep increase in life expectancy during the $20^{\text {th }}$ century. ${ }^{[1]}$ This remarkable success was set into motion by the serendipitous discovery of penicillin in 1928 by Sir Alexander Fleming. ${ }^{[2]}$ After the successful purification of penicillin G in 1942, the so called "golden era" of antibiotics began, marked by the discovery of many novel antibiotics between the 1950s and 1970s. ${ }^{[3]}$ Since then, the approach has shifted to the screening of derivates and the modification of existing antimicrobial agents, and, while new drugs are brought to market frequently ${ }^{[4]}$, overall approval of new antibiotics has significantly decreased.[5] New classes of antibiotics for widespread use have not been developed in the last decades. ${ }^{[6]}$

Due to their irreplaceable role in the treatment of infections, antibiotics are ubiquitously used to treat humans and livestock. However, this prevalence in combination with frequent misuse has led to the emergence of a wide variety of drug-resistant bacteria strains in recent decades and resistances have been reported for nearly all antibiotics in use today. ${ }^{[7][8]}$ The WHO estimates that infections with drug-resistant pathogens cause at least 700,000 deaths per year worldwide. ${ }^{[9]}$ Especially multidrug-resistant strains are of major concern, with methicillin-resistant S.aureus (MRSA) being the most prominent, but by far not the only challenge to effective infection control with antibiotics. ${ }^{[10]}$

The quest for novel antimicrobial compounds is thus a vital area of research. A wide variety of approaches are currently under investigation, including the use of bacteriophages, ${ }^{[1]}$ phage endolysins, ${ }^{[12]}$ antibodies, ${ }^{[13]}$ and macrocyclic peptidomimetics. ${ }^{[14]}$ A further promising approach is to investigate naturally occurring antibiotics found in animals, plants and microorganisms, such as antimicrobial peptides ${ }^{[15]}$, nucleoside antibiotics ${ }^{[16]}$ or peptidoglycan recognition proteins. ${ }^{[17]}$ 


\subsection{Antimicrobial peptides}

Antimicrobial peptides (AMPs) usually directly target the bacterial cell membrane. This modus operandi distinguishes them from classical antibiotics which target the cell wall synthesis or the cells' metabolic processes. ${ }^{[18]}$ The potency of the AMPs' mechanism of attack can be seen in daptomycin, a cyclic lipopeptide that depolarizes Gram-positive bacteria, which is used to great effect in the treatment of infections with multidrug-resistant bacteria. ${ }^{[19]}$ Most AMPs are small linear amphipathic peptides with a length of typically less than 40 amino acids, structured as $\alpha$-helix, $\beta$-strands or a mix thereof. Non-helical and cyclic peptides are also found. ${ }^{[20]}$

The AMPs' mode of action can include intracellular effects, but usually is characterized by the permeabilization of the plasma membrane through binding to it, aggregating, and inducing membrane defects. ${ }^{[21]}$ The first interaction with the negatively charged bacterial membrane is usually mediated by electrostatics, as many AMPs carry multiple positive charges. Nevertheless, anionic peptides can also interact with bacterial membranes, as seen with dermcidin (DCD) for example, which forms oligomeric pores in the presence of $\mathrm{Zn}^{2+}$ ions. ${ }^{[22]}$

Several mechanisms for the membrane permeabilization by AMPs have been proposed and found. ${ }^{[23]}$ By partial insertion of amphiphilic helixes into the bilayer, toroidal pores can be introduced into lipid bilayers. The insides of these pores are lined by the hydrophilic headgroups of the lipids itself, stabilized by the peptides. Another possible way of pore formation is the vertical insertion of peptides to form a pore completely lined by peptides, the so called barrel stave model. ${ }^{[24]}$ Less structured permeabilization is characterized by an accumulation of peptide on the membrane surface, followed by a detergent-like lysis of the bilayer after a threshold concentration is reached (carpet model).[25] This process can be assisted by intrinsic negative curvature in bilayers, which is compensated by peptides and leads to increased accumulation. ${ }^{[26]}$ 


\section{Introduction}

The AMPs' mode of action can be studied in a variety of lipid model systems, ranging from micelles and unilamellar vesicles to planar systems like Langmuir monolayers, solid supported membranes and pore-spanning membranes ${ }^{[27,28]}$

\subsection{Muraymycin}

Nucleosides are ubiquitous in all forms of life, not only as the coding subunits of DNA and RNA, but also in virtually all areas of the living cell. They play a crucial role in the cell metabolism as part of energy carriers or coenzymes (e.g. ATP, NAD, FAD, UDP) as well as in cell signaling (e.g. cAMP, cGMP). It is, therefore, not surprising that nucleoside structures are being investigated as pharmacological drugs and have been found to be a useful tool in medicine. Cytotoxic nucleoside analogues, for example, are used in the treatment of cancer ${ }^{[29]}$ and nucleoside reverse-transcriptase inhibitors are an essential part of antiretroviral therapy. ${ }^{[30]}$

Naturally occurring nucleoside antibiotics were first described in the $1960 \mathrm{~s}^{[31]}$, and a large catalogue of classes has been compiled since then. ${ }^{[32]}$ Their modes of action include the inhibition of nucleic acid synthesis and bacterial enzymes, both in protein and cell wall synthesis. ${ }^{[16]}$ The issue of antibiotic resistance has led to an increased interest in nucleoside antibiotics and their potential use in treating infections. ${ }^{[33,34]}$

Peptidoglycan is the structural element of bacterial cell walls, protecting bacteria against environmental and osmotic stress. It is comprised of a polysaccharide of alternating Nacetylglucosamine (GlcNAc) and N-acetylmuramic acid (MurNAc) units, which is crosslinked via pentapeptide sidechains of MurNAc. Its synthesis is the target of many drugs, including the classic penicillins, which inhibit the enzymes responsible for the transpeptidation involved in cross-linking. ${ }^{[35,36]}$ Many nucleoside antibiotics, e.g. tunicamycin, mureidomycin, and pacidamycin, inhibit the enzyme MraY, a translocase playing a crucial role in the lipid-linked coupling of GlcNAc and MurNAc-pentapeptide. ${ }^{[33]}$ Specifically, MraY is responsible for the linking of UDP-MurNAc-pentapeptide to a 
polyprenyl phosphate carrier-lipid and thereby fixating it at the membrane for the coupling reaction catalyzed by the glycosyltransferase MurG. ${ }^{[37]}$

One promising new class of nucleoside antibiotics that target MraY are the muraymycins that have been isolated from Streptomyces sp. LL-AA896. ${ }^{[38]}$ Muraymycin consists of a uronic acid core derived from uridine, which is 5'-O-aminoribosylated in most muraymycins. The uridine moiety is attached via an alkyl linker to a peptide with distinct features: The peptide is terminated by a urea-valine motif and contains an unusual amino acid, Lepicapreomycidine. ${ }^{[39]}$ These features, an effective reversal of peptide directionality by urea next to a cyclic arginine analogue, have also been found in proteases inhibitors like chymostatin. ${ }^{[40]}$

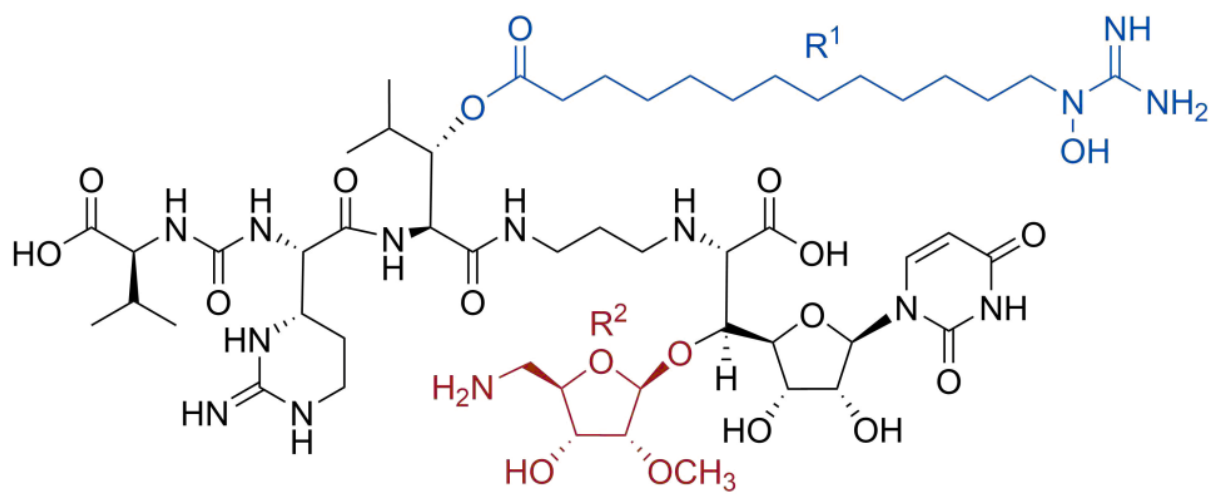

Figure 1.1 Structure of Muraymycin A1. The lipid side chain is depicted in blue $\left(\mathrm{R}^{1}\right)$, the aminoribose moiety in red $\left(\mathrm{R}^{2}\right)$.

The muraymycins are differentiated into four series, which are defined by the fatty acid moiety attached to the peptide at the central leucine. The sidechain decreases in length from A- to B-series, and is completely absent in the $\mathrm{C}$ - and $\mathrm{D}$-series $(\mathrm{OH}$ - and $\mathrm{H}$ - terminated, respectively). The series are further differentiated by the group terminating the lipid sidechain and the amino sugar attached to the uronic acid. [38]

Both the aminoribose moiety and the length and functionalization of the lipid sidechain have been found to significantly influence antibacterial activity of the muraymycins in structureactivity relationship (SAR) studies. ${ }^{[38,41,42]}$ The aminoribose has been found to be essential for MraY inhibition, but it has been suggested that cellular uptake and possibly efflux phenomena influence the magnitude of antibacterial activity of muraymicins. ${ }^{[43]}$ The function 


\section{Introduction}

of the lipid sidechain is believed to be mainly related to the uptake, as sidechain composition influences the antibacterial activity in S.aureus ${ }^{[38,44]}$ but not the MraY inhibition. ${ }^{[43]}$

Understanding the workings of nucleoside antibiotics in general and muraymycin in particular is an important step in advancing the research into their use as pharmaceutical drugs. Thus, this thesis aimed to provide insight into the role of the fatty acid sidechains in the activity of muraymycin, particularly its cellular uptake.

\subsection{Lugdunin}

The human body is colonized by a large number of bacteria, archae, fungi and viruses, forming a rich microbiome of mutualistic, pathogenic, and commensal microorganisms. Commensal bacteria offer no direct benefit or harm to the host. However, they can support the host's defense against pathogens by competing with them for nutrients and by inducing immune reactions. ${ }^{[4]}$ Furthermore, many commensal organisms produce bacteriocins, that is antimicrobial substances, that are not harmful to the commensals themselves. ${ }^{[46-48]}$ It has therefore been proposed to combat infections of drug-resistant bacteria by utilizing probiotics derived from naturally occurring commensal microbiota. ${ }^{[49]}$ The bacteriocins produced by commensal bacteria in the human body also provide a valuable range of promising antimicrobial substances that could be used to develop new antibiotics. ${ }^{[50]}$

Recently, one such novel antibacterial peptide was identified in nasal Staphylococcus lugdunensis. ${ }^{[51]}$ While $S$. lugdunensis can cause mild to severe infections similar to $S$. aureus infections, ${ }^{[52]}$ it usually is considered as commensal, occupying niches of the normal skin flora. ${ }^{[53]}$ It was found to compete successfully with $S$. aureus in the human nose. ${ }^{[51,54]}$ Screening nasal Staphylococcus isolates for antimicrobial activity has shown the strain $S$. lugdunensis IVK28 to strongly inhibit growth of S. aureus. Mutagenesis experiments traced the activity to a non-ribosomally synthesized peptide, which was named lugdunin. ${ }^{[51]}$

Lugdunin is a cyclic pentapeptide containing hydrophobic amino acids in alternating D- and L- configuration. The ring is closed by a thiazolidine moiety, which is believed to be formed 


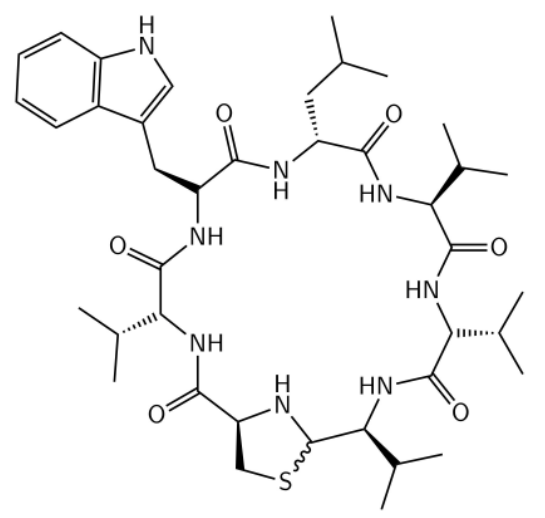

Figure 1.2: Structure of lugdunin

by the condensation of an N-terminal cysteine with a Cterminal valine from a linear heptapeptide precursor. ${ }^{[51]}$

The mode of action of the peptide is not yet fully understood. Besides directly Scheme 1.1: Lugdunin inhibiting $S$. aureus, lugdunin has been shown to induce an innate immune response against $S$. aureus in the host, amplified by factors from other commensals. ${ }^{[55]}$ Furthermore, a synergistic effect with host-derived antimicrobial peptides was found. Simultaneous incubation with dermcidin, an AMP secreted on the human skin, leads to increased activity against $S$. aureus in comparison to the sum of both separate peptide activities or even sequential treatment. ${ }^{[55]}$

A critical step towards understanding these processes is the investigation of the peptidemembrane interaction.

\subsection{Membrane model systems}

Biological cell membranes can be modeled with natural lipid extracts or artificial lipids, greatly simplifying the characterization of membrane-specific processes. Many amphipathic lipid molecules spontaneously aggregate in aqueous environments to form lipid bilayers due to the entropy-driven hydrophobic effect. Depending on the specific lipids, these artificial bilayers can show similar features to their natural counterpart, e.g. fluidity, charge, or mechanical properties, while constituting a "clean slate" for observing membrane bound molecules and isolated interactions between a lipid membrane and a substance of interest. This approach is widely used to investigate active and passive ion channels, protein-induced membrane fusion, components of the cytoskeleton, and membrane interacting proteins in general. In the context of antimicrobial peptides, model membranes are a valuable tool for characterizing the mode of action of a peptide, which usually involves the permeabilization of the lipid bilayer in one form or another. 


\section{Introduction}

The lipid bilayers can be readily obtained in the form of spherical vesicles (liposomes) by various techniques. These constitute small, closed aqueous compartments and allow the investigation of membrane fusion, transport processes, and binding of molecules in bulk assays. When vesicles are immobilized via spreading onto a solid surface, the lipid bilayers can be used in conjunction with surface sensitive measurement techniques like surface plasmon resonance (SPR) or reflectometric interference spectroscopy (RIfS) to observe the binding of proteins or small molecules. Morphology and mechanical properties of the membrane and bound proteins can be probed via atomic force microscopy (AFM) in these 2D-systems. Transmembrane processes, however, can only be accessed to a limited extent in solid-supported lipid bilayers, e.g. by electrochemical impedance spectroscopy, as only a very thin water layer separates the bilayer from the substrate.

To combine the advantages of a fixed 2D-system with the requirement of aqueous compartments on both sides of the bilayer, pore-spanning membranes (PSMs) can be employed. By spreading a bilayer on a porous substrate, freestanding membranes (f-PSMs) are held in a defined geometry by the solid-supported parts of the membrane (s-PSM), allowing the use of techniques like AFM or confocal microscopy to investigate membrane properties and interactions. ${ }^{[56-58]}$

For the investigation of membrane permeabilization, such a PSM model system was developed on the basis of a porous alumina substrate. ${ }^{[28]}$ The use of nano-porous anodic aluminium oxide (AAO) has the distinct advantage that it is optically transparent, which allows access to the aqueous compartment beneath the bilayer not only by fluorescence microscopy, but also optical surface sensitive techniques like RIfS[59] or optical waveguide spectroscopy Figure 1.3: Illustration (OWS).[60][61]

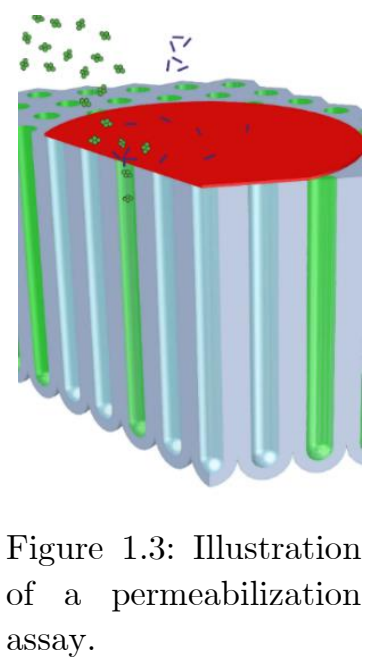

The permeabilization of lipid bilayers by AMPs is directly observable by confocal fluorescence microscopy as the influx of fluorescent dyes into the closed pores beneath the f-PSMs. In contrast to bulk vesicle assays, where permeabilization is usually measured as the release of specific dyes in high, self-quenching concentrations, a wide variety of fluorescent molecules can be employed. This permits, for example, the use of large dextran- 
bound fluorophores to probe the diameter of membrane defects. Moreover, the peptide induced removal of lipids from the substrate, following the carpet model, can be directly observed via fluorescently labeled lipids. ${ }^{[62]}$

The 2D approach of PSMs on a fixed substrate has great potential for the development of highly integrated "lab-on-a-chip" assays. ${ }^{[63]}$ The permeabilization assay with PSMs on a AAO substrate constitutes a promising tool for the screening of antimicrobial substances for membrane permeabilization and for the characterization of their interaction. Moreover, the substrate can be readily modified to include novel features. The introduction of metal electrodes, for example, would allow the application of a transmembrane potential across the pore-spanning membranes and thus further refine the model to more closely mimic fundamental properties of a bacterial plasma membrane. 


\section{Scope}

Membrane model systems are a valuable tool for the investigation of specific interactions of lipid bilayers with peptides or proteins, isolated from unwanted influences. In this thesis, the interaction of two antimicrobial compounds with bacterial membranes is modeled in different systems.

The nucleoside antibiotic muraymycin shows variations in antibacterial activity depending on the lipid sidechains attached to the peptide. Fluorescence labeled derivates of these sidechains are investigated by incubating adhered giant unilamellar vesicles (GUVs) with them. By monitoring the dye translocation into the membrane and the GUV interior via confocal fluorescence microscopy, conclusions about the role of these sidechains in the activity of muraymycin are drawn.

Furthermore, the mode of action of the cyclic peptide lugdunin is elucidated in assays utilizing unilamellar vesicles in bulk. To gain insight into the mechanisms governing lugdunin's depolarization of bacterial cells, separate fluorescence-based experiments are conducted, testing the propensity of lugdunin to cause unspecific permeabilization of lipid bilayers or ion transport across them.

In addition to measurements with established membrane model systems, a permeabilization assay based on pore-spanning membranes is further developed by embedding silver electrodes

into the porous substrate. The aim of this modification is the ability to directly apply a transmembrane potential during the membrane's permeabilization by antimicrobial compounds. Fabrication procedures for this assay are developed and the application of a transmembrane potential is explored in proof-of-principle experiments. 


\section{Materials and Methods}

A biological membrane is a complicated system. Thus, membrane processes can often be studied more conveniently in artificial lipid bilayers which act as a simplified model for the membrane, removing all unwanted interactions with membrane bound proteins and scaffolds. Such model systems for the bacterial cell membrane form the basis of all experiments in this thesis ${ }^{[64,65]}$.

\subsection{Lipids and Vesicles}

The lipid bilayers employed in this thesis varied in composition and morphology. Most proofof-principle measurements were conducted with bilayers composed entirely of 1-Palmitoyl-2oleoyl-sn-glycero-3-phosphocholine (POPC). Bilayers prepared from this zwitterionic, partially unsaturated lipid showed overall good stability in vesicles and pore-spanning membranes. The use of a generic single lipid component has the advantages of decreasing the variability between preparations and reducing the model system complexity to a bare minimum. To mimic the membrane composition of bacterial cells more closely, the lipids 1Palmitoyl-2-oleoyl-sn-glycero-3-phosphoethanolamine (POPE) or 1-palmitoyl-2-oleoyl-snglycero-3-phosphoglycerol (POPG) were added in varying amounts to POPC in some experiments, as specified below. ${ }^{[66]}$ POPE has a smaller headgroup region than POPC, which influences packing and hydration of the bilayer. ${ }^{[67]}$ In POPG the negative charge of the phosphate-group is not compensated by an amino-group, leading to an overall negatively charged lipid. All lipids were purchased from Avanti Polar Lipids (Alabaster, AL, USA). 
POPC

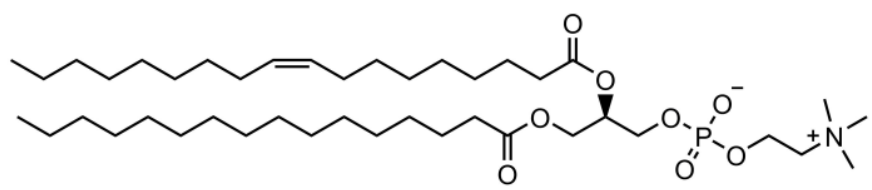

POPE

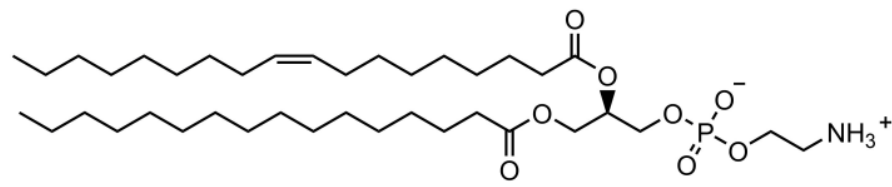

POPG

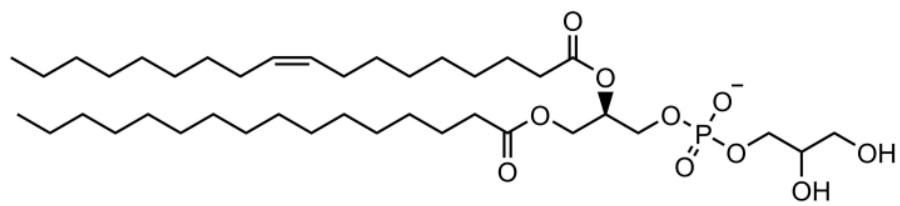

Figure 3.1: Structures of the phospholipids POPC, POPE and POPG.

For the experiments with phase-separated membranes, a ternary mixture of 1,2-Dioleoyl-snglycero-3-phosphocholine (DOPC), Sphingomyelin (SM, Brain, porcine) and cholesterol was used.

DOPC
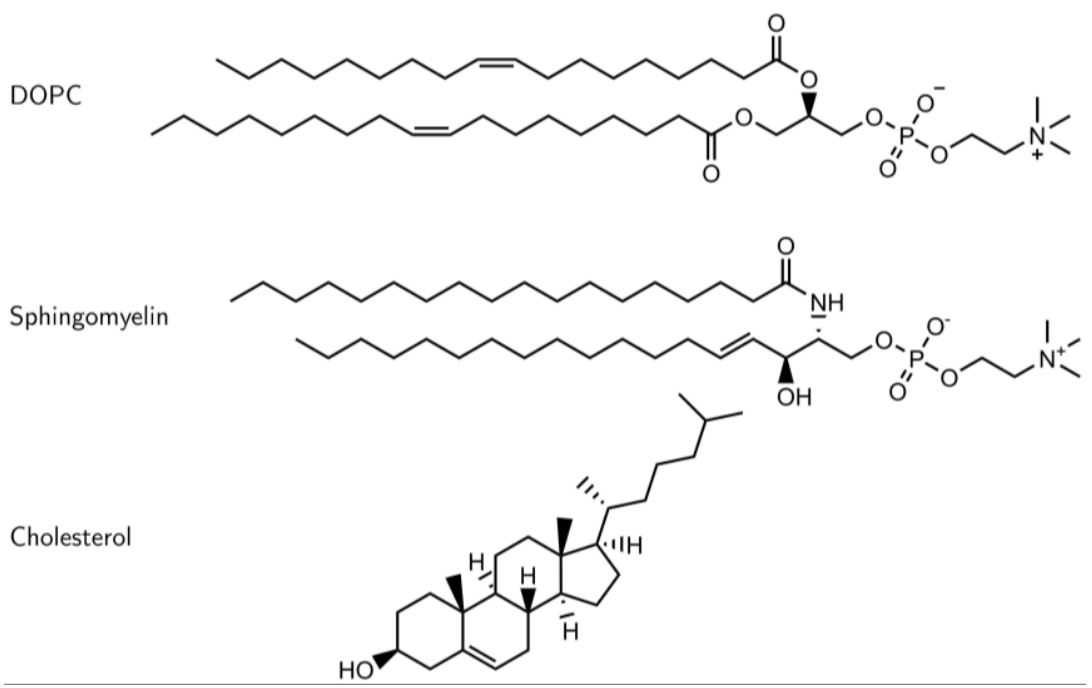

Figure 3.2: Structures of DOPC, 18:0 SM, the main component in the SM used, and cholesterol

For bulk measurements, bilayers were prepared in the form of small unilamellar vesicles (SUVs, mean diameter $<0.1 \mu \mathrm{m}$ ) or large unilamellar vesicles (LUVs, mean diameter 0.1$1 \mu \mathrm{m})$. For fluorescence microscopy-based experiments, giant unilamellar vesicles (GUVs, diameter 5 to $>100 \mu \mathrm{m})$ were prepared. All preparations started from lipid solutions of known 


\section{Materials and Methods}

concentration in chloroform, stored at $-20{ }^{\circ} \mathrm{C}$. Lipid films obtained from these solutions were always thoroughly dried before rehydration, ensuring solvent-free bilayers.

\subsubsection{Preparation of SUVs}

Small unilamellar vesicles were freshly prepared via ultrasonic homogenization of lipid material suspended in buffer. To this end, lipids dissolved in chloroform were dispensed into glass vials and dried in a focused nitrogen stream to form a lipid film adhering to the glass walls. After further drying in a vacuum for at least $1 \mathrm{~h}$, buffer was added to the vial. After a 30 min swelling period, the lipid material was resuspended into the solution by thorough agitation on a vortex mixer for $3 \mathrm{x} 30$ s. The suspension was transferred into an Eppendorf cup and homogenized in a high-powered ultrasonic bath for $20 \mathrm{~min}$. (amplitude 50 \%, duty cycle $40 \%$, Sonopuls HD2070, Bandelin, Germany).

\subsubsection{Preparation of LUVs}

Large unilamellar vesicles were prepared via extrusion. First, as described for the SUV preparation, lipid films were obtained by dispersing and drying a lipid solution in a nitrogen stream. The vacuum dried films were swelled in buffer for $30 \mathrm{~min}$ and resuspended by thorough agitation on a vortex mixer for 3x 30 s. Subsequently, the lipid suspension was extruded 31 times through a polycarbonate membrane with a nominal pore diameter of $200 \mathrm{~nm}$ in a small, syringe driven extruder (LiposoFast, Avestin, Canada). If required, the buffer surrounding the vesicles was exchanged via size exclusion chromatography with prepacked Sephadex G-25 columns (illustra NAP-25, GE Healthcare Life Science). As approximately $50 \%$ of lipid material is lost in the process of extrusion and gel filtration, the actual lipid concentration of the LUVs suspension was subsequently determined with a phosphate test.

For this test, $30 \mu \mathrm{L}$ samples of the LUVs suspension as well as calibration samples of known $\mathrm{NaH}_{2} \mathrm{PO}_{4}$ concentration were heated with $200 \mu \mathrm{L}$ perchloric acid $(70 \%)$ to $220{ }^{\circ} \mathrm{C}$ for $1 \mathrm{~h}$. The inorganic residues were dissolved in $700 \mu \mathrm{L} 12.6 \%(\mathrm{w} / \mathrm{v}) \mathrm{HClO}_{4}$ containing $0.45 \%$ (w/v) 
$\mathrm{NH}_{4} \mathrm{MoO}_{4}$, then $700 \mu \mathrm{L}$ of a $1.7 \%(\mathrm{w} / \mathrm{v})$ ascorbic acid solution were added. Subsequently, the solution was incubated for $5 \mathrm{~min}$ at $80{ }^{\circ} \mathrm{C}$ and the absorption of the samples at $820 \mathrm{~nm}$ was measured via spectrophotometry. Phosphor concentration was then calculated from the calibration curve.

\subsubsection{Preparation of GUVs}

Giant unilamellar vesicles were prepared via electroformation. ${ }^{[68,69]}$ Lipid films were prepared directly on ITO-coated glass slides by pipetting $15 \mu \mathrm{l}$ of lipids dissolved in chloroform on each slide and dispersing the drops manually with the pipette tip. After drying in a vacuum for at least $1 \mathrm{~h}$, two slides each were assembled with a silicone spacer to form a small chamber approx. $1.7 \mathrm{ml}$ in volume. The chamber was then filled with sucrose solution equal-osmolar to the buffer used in subsequent experiments, while the slides were immediately connected to a signal generator providing an alternating voltage with a frequency of $12 \mathrm{~Hz}$ and an amplitude of $1.6 \mathrm{~V}_{\mathrm{pp}}$. After $3 \mathrm{~h}$ the signal generator was disconnected and the vesicle suspension transferred to a light-proof reaction cup via pipette. The vesicle suspension was stored at room temperature.

Phase-separated GUVs were prepared similarly to normal GUVs, but drying of the lipid films and electroformation were conducted above the transition temperature of around $40{ }^{\circ} \mathrm{C}$, above which the lipids mix into one homogeneous phase. ${ }^{[70]}$ To achieve this result, the ITO slides were dried in a heated vacuum chamber and the sucrose solution was preheated to $55{ }^{\circ} \mathrm{C}$. After drying, the warm slides were swiftly assembled, filled with preheated buffer and placed in an oven for electroformation at $55{ }^{\circ} \mathrm{C}$. To reduce evaporation through the small gap at the top of the silicone spacer, a small open beaker filled with water was placed into the oven. After $3 \mathrm{~h}$, the vesicle suspension was transferred from the ITO chamber to a reaction cup while still heated, but subsequently stored at room temperature. 


\section{Materials and Methods}

\subsection{Fluorescence}

The methods used in this thesis are based on the principle of fluorescence, either as a tool for localizing structures in microscopy, as a quantitative probe for transport or chemical processes, or a combination thereof. Fluorescence is the spontaneous emission of light by molecules after excitation by photons. The emitted light is red-shifted (Stoke-shift) and can thus be distinguished from excitation light and scattered light through optical means like diffraction gratings or dichroic mirrors. Fluorescing molecules, called fluorophores, usually exhibit extended $\pi$-electron systems with excitation wavelengths from the near UV to visible light. After excitation, they lose some of the energy via vibrational relaxation, both in the fluorophore itself and in the surrounding solvent, as the excited state usually shifts the dipole moment of the molecule. The excitation energy can also be directly transferred to a second fluorophore in close proximity via non-radiative dipole coupling. This Förster resonance energy transfer (FRET) from a donor to an acceptor dye with overlapping emission and excitation spectra, respectively, is highly dependent on the distance between the fluorophores and can be used to measure inter- and intramolecular distances. ${ }^{[71]}$

Fluorophores can be chemically coupled to proteins or lipids to make them localizable and quantifiable. To observe lipid bilayers via microscopy, lipid-coupled fluorophores are used. The membrane probes used in this thesis were the headgroup-labeled phospholipid TexasRed-DHPE and the cholesterol derivate BODIPY-chol.

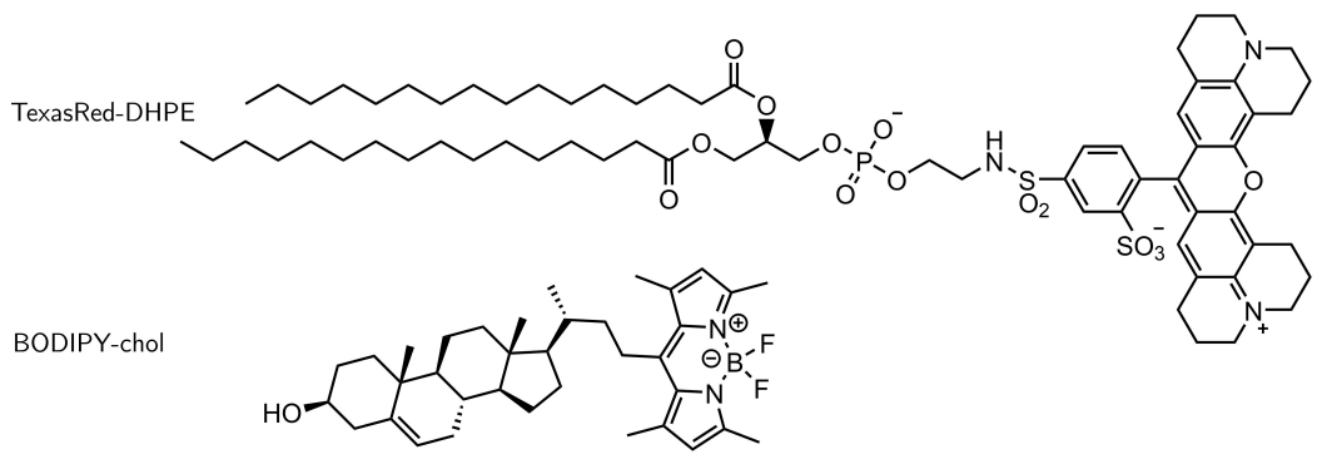

Figure 3.3: Structures of membrane marker dyes used in this thesis. 
The labeled lipid sidechain derivates used in chapter 4.1 were kindly provided by the Ducho group of the Saarland University. They were obtained from the propargyl esters of the lipids via click-reaction with AlexaFluor488-azide (AF488).[72]

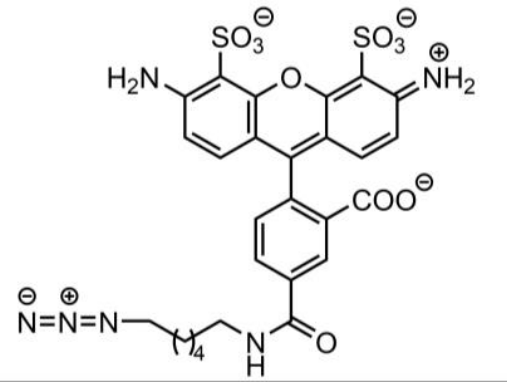

Figure 3.4: Structure of the AF488 derivate used to label lipid sidechains

As water soluble fluorescent dyes, 8-hydroxypyrene-1,3,6-trisulfonic acid (HPTS, pyranine), 5(6)-carboxyfluorescein (CF), and 9-amino-6-chloro-2-methoxyacridine (ACMA) were used.
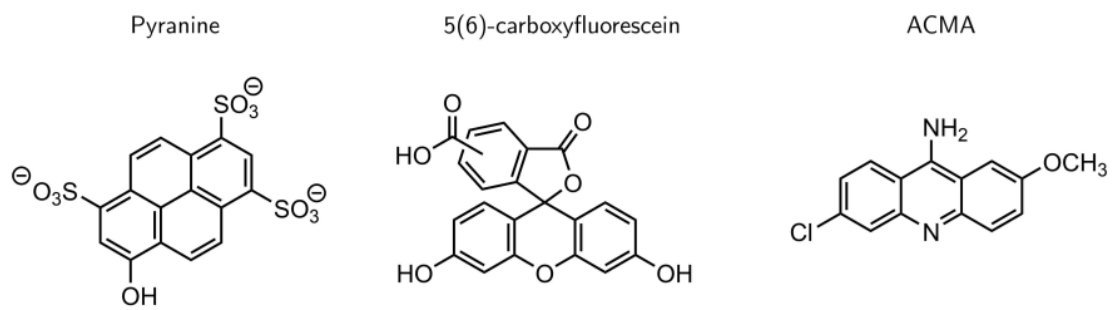

Figure 3.5: Structures of fluorescent dyes used in aqueous solution.

To visualize transmembrane potentials, lipid bilayers were incubated with $3,3^{\prime}$ diethyloxacarbocyanine iodide (DiOC (3)).

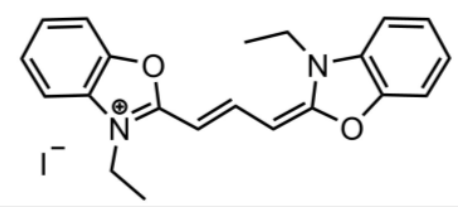

Figure 3.6: Structure of $\mathrm{DiOC}_{2}(3)$. 


\subsubsection{Fluorescence spectrometry}

The fluorescence spectra and intensities of solutions and liposome suspensions were measured with a standard fluorescence spectrophotometer. Here, a beam of monochromatic light is focused on the sample and the emitted light is collected perpendicular to the excitation light and detected by a photomultiplier tube (see Figure 3.7). This setup allows the characterization of very dilute samples, as it directly measures the integrated signal of a volume of micro- to milliliters. All samples were measured in a fused quartz cuvette with a solution cross-section of $10 \times 4 \mathrm{~mm}^{2}$ and were stirred by a small magnetic stir bar. Time course measurements were low pass filtered with a time constant of $0.1 \mathrm{~s}$ and recorded with a resolution of $1 \mathrm{~s}$. The excitation and emission bandwidths were set to $5 \mathrm{~nm}$. Spectra were measured with $3 \mathrm{~nm}$ bandwidth for the scanned parameter and $3 \mathrm{~nm}$ to $20 \mathrm{~nm}$ for the fixed.

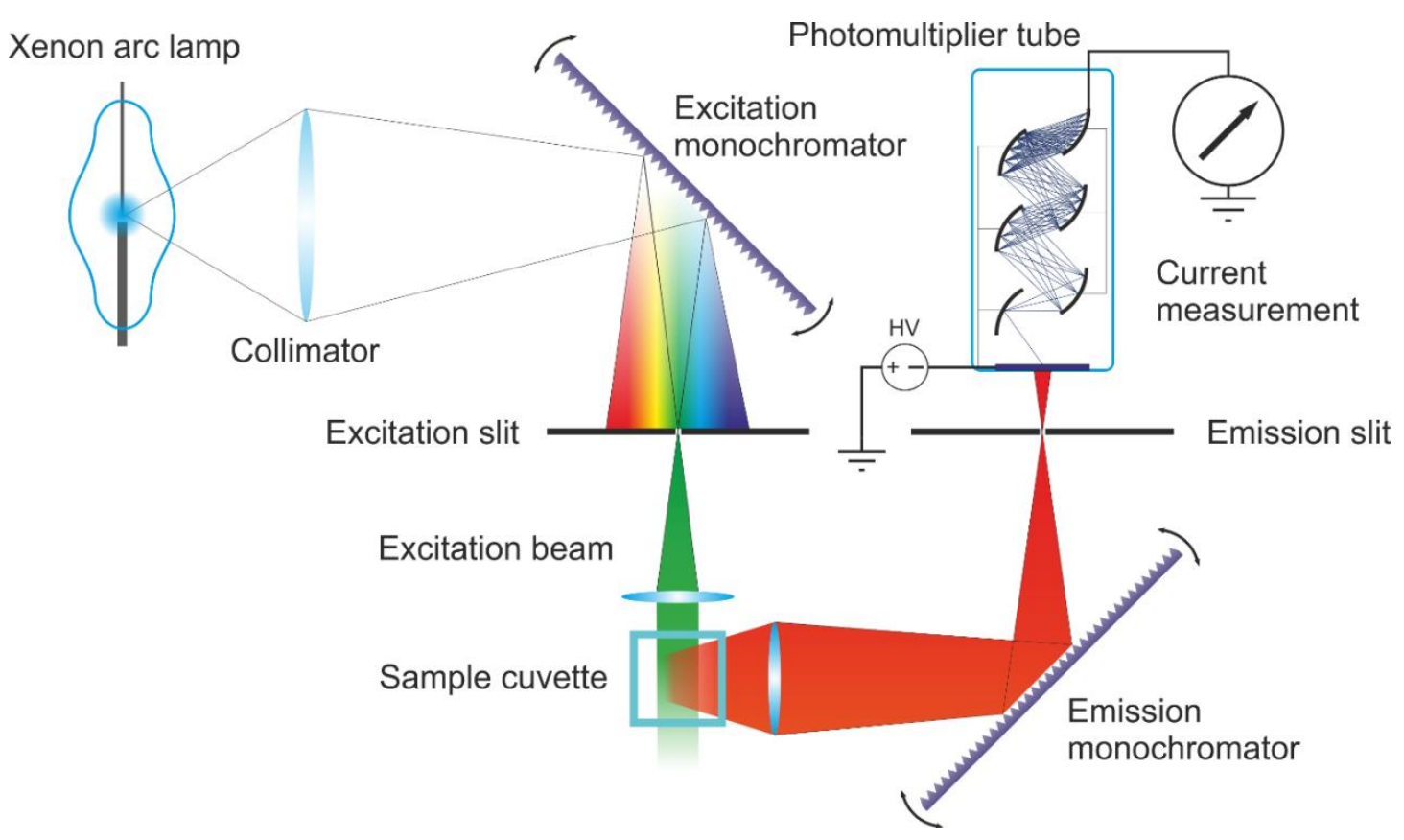

Figure 3.7: Schematic diagram of a fluorescence spectrophotometer. Light from a high intensity light source, e.g. a xenon gas discharge lamp, is focused onto the adjustable excitation monochromator, consisting of a rotatable diffraction grating and a slit. The slit width is adjustable and defines the bandwidth of the excitation light. The monochromatic beam is collimated and enters the sample compartment. Emitted light is collected at a right angle, filtered by the emission monochromator, and converted to a current by a photomultiplier tube. The current is amplified and recorded as the fluorescence signal. The sensitivity is adjusted via the high voltage across the tube. 


\subsubsection{Fluorescence microscopy}

The labeling of small structures with fluorescent molecules or nanoparticles permits their observation with a multitude of different microscopy techniques. The simplest fluorescence microscopes focus light onto the sample through a dichroic filter, which passes the red-shifted emission light that is collected by the objective to the ocular or a camera. This widefield "epifluorescence" microscope setup can image 2D samples with good resolution. However, fluorescence is also excited above and below the focal plane, resulting in a diffuse glare when observing 3D structures. For single, well defined structures such as free GUVs, the off-plane signal is also well defined and can be utilized to quantify absolute fluorescence. In more complicated structures and when no knowledge about the properties in the z-direction is available, the usable resolution decreases significantly. The observation of structures in a fluorescent solution is not possible, as the stray light from the whole illuminated volume is collected and completely drowns out any other signal.

There are two approaches permitting the observation of three-dimensional samples. The first is to selectively excite fluorescence in the focal plane only. One example for this approach is light sheet microscopy, where the sample is illuminated only by a thin slice of excitation light perpendicular to the z-axis. This technique suppresses unwanted fluorescence from outside the focal plane but is limited by the geometry of the setup and the restrictive requirements it places on the samples. More versatile are techniques where illumination and detection are both performed along the z-axis via the microscope objective. The second approach, therefore, is to excite fluorescence parallel to the observation axis but to block any emitted light originating from outside the focal plane. This can be achieved by refocusing the emitted light and directing it through a small aperture that is "confocal" to the sample. Light from other planes is mostly blocked by the pinhole plate, as its focus point lies in front or behind the confocal plane. The downside of confocal microscopes is that no $2 \mathrm{D}$ information can directly pass the pinhole and the image must be acquired by sequentially scanning the sample point by point. The sequential image acquisition can be implemented either by moving one or several pinholes through the light path, as is done with spinning disc microscopy, or by scanning the light path across the sample with mirrors and collecting the signal through a fixed pinhole, as is done with confocal laser scanning microscopy (CLSM, 
see Figure 3.8). The resolution of a CLSM in the xy-direction is governed by the numerical aperture of the objective and the excitation and emission wavelengths. The z-resolution is generally lower and is also dependent on the pinhole diameter. The 3D-resolution of a CLSM is described by its point-spread function (PSF), the image obtained from an ideal point source.

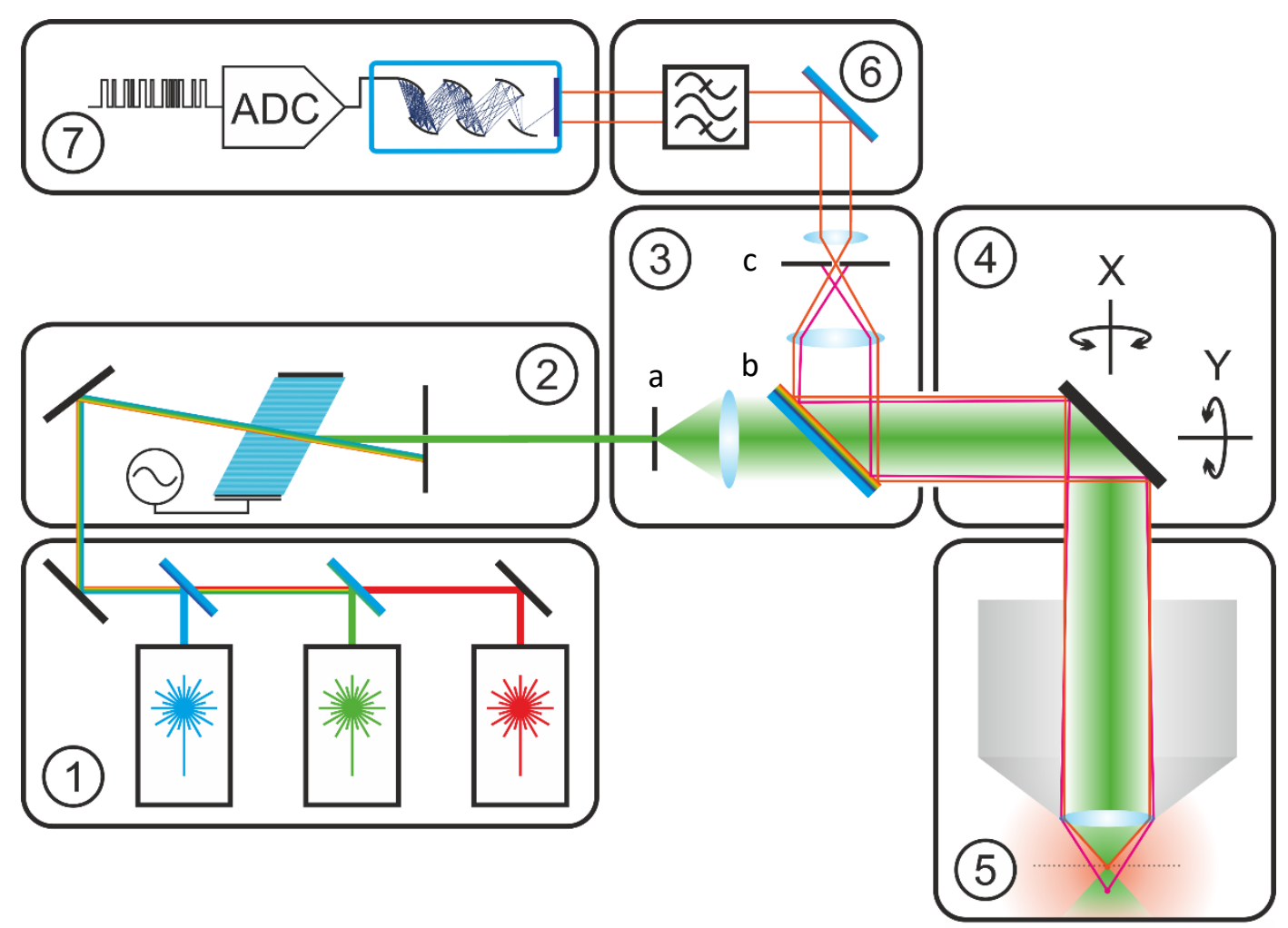

Figure 3.8 Simplified schematic of a confocal laser scanning microscope. Monochromatic light is generated by lasers and combined into a single beam via dichroic mirrors (1). The beam is passed through an acousto-optic tunable filter (2), where the intensity of each excitation wavelength is modulated individually. Soundwaves generated by a piezo transducer travel through a crystal, inducing variations in refractive index which deflect selected wavelengths by diffraction. The excitation beam enters the microscope light-path through a confocal aperture (3a), passes the beamsplitter (3b) and is guided by the scanning mirror (4) to the objective (5). The light is focused to a point in the focal plane and scanned across the sample. Emitted light of longer wavelength is collected by the objective and reflected onto the confocal pinhole (3c) by the beam-splitter. The pinhole plate blocks light from outside the focal plane. Light passing the pinhole is guided through a spectral bandpass (6) to the detector, usually a photomultiplier tube (7). The time-dependent electrical signal generated by the detector is then used to reconstruct the image. 


\subsection{Adhered GUVs}

Adhered GUVs provide an excellent model system for characterizing the interaction between fluorescently labeled molecules in aqueous solution and lipid membranes. The size of GUVs ranging from tens to hundreds of micrometers is sufficiently large to permit the direct measurement of fluorescence in both the lipid bilayer and the aqueous interior of the vesicles via confocal microscopy. Adhering the vesicles to a solid substrate via functionalized lipids allows the observation of individual vesicles over long periods of time as well as the exchange of the surrounding solution.

Fluorescence measurements were performed with a water immersion objective in an upright microscopy setup. A conical measurement chamber was designed to minimize the volume of aqueous solution required (see Figure 3.9). The substrate is clamped between the upper conical chamber and a base plate. A thin silicone ring, cast from PDMS into an aluminium mold, sits in a recess of the upper chamber and seals the interface to the substrate.

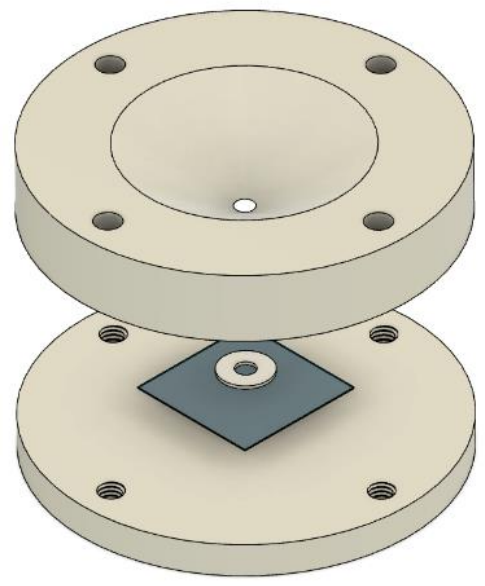

Figure 3.9: Schematic view of the conical chamber used for low-volume CLSM measurements.

\subsubsection{GUV adhesion}

The controlled adhesion of vesicles was realized by functionalizing a small fraction of the lipids with biotin and the substrate surface with the protein avidin. The specific interaction between biotin and avidin is non-covalent, but nearly as strong. ${ }^{[73]}$ Two different methods were used to obtain avidin functionalized substrates. At first, hydrophilized silicon wafers were directly incubated with avidin, whereas later a more sophisticated method using biotinfunctionalized glass cover slips was employed. Due to the four binding sites for biotin on avidin, a biotin covered surface can be saturated with avidin which then still presents sites for further binding of biotinylated lipid bilayers. In most experiments, instead of the 


\section{Materials and Methods}

naturally occurring glycoprotein avidin the deglycosylated modification neutravidin was used, which has an isoelectric point near neutral and shows less unspecific binding.

For the direct avidin adsorption, silicon wafers were wiped with iso-propanol, cleaned in a bath containing $\mathrm{H}_{2} \mathrm{O}: \mathrm{NH}_{3}(29 \%): \mathrm{H}_{2} \mathrm{O}_{2}(30 \%)(5: 1: 1)$ at $70{ }^{\circ} \mathrm{C}$ for 20 min and rinsed with water several times. After mounting in the measuring chamber, the wafer was incubated with $50 \mu \mathrm{M}$ avidin in PBS buffer $\left(137 \mathrm{mM} \mathrm{NaCl}, 2.7 \mathrm{mM} \mathrm{KCl}, 1.5 \mathrm{mM} \mathrm{KH} 2 \mathrm{PO}_{4}, 8.1 \mathrm{mM}\right.$ $\mathrm{Na}_{2} \mathrm{HPO}_{4}$, pH 7.4) for $1 \mathrm{~h}$. The chamber was then rinsed with buffer several times.

For the binding of avidin on biotin-coated surfaces, class cover slips were covalently functionalized with polyethylene glycol (PEG), containing $10 \mathrm{~mol} \%$ biotin-terminated polymer chains. ${ }^{[74]}$ First, the glass slides were thoroughly cleaned as described above, dried and cleaned with oxygen plasma (1 min at $60 \%$ power, Zepto LF PC, Diener electronic, Ebhausen, Germany). Immediately after, the slides were coated with a thin film of 3glycidyloxypropyltrimethoxysilane (GOPTS). Several slides were stacked to reduce the influence of atmospheric water vapor and heated for $1 \mathrm{~h}$ to $80{ }^{\circ} \mathrm{C}$. After incubation, the slides were separated, rinsed with acetone and dried in a nitrogen stream. A mixture of $90 \mathrm{~mol} \%$ $\alpha$-methoxy- $\omega$-amino-PEG (2,000 Da) and 10 mol\% $\alpha$-amino- $\omega$-biotin-PEG (3,000 Da) was melted in a thermomixer at $85{ }^{\circ} \mathrm{C}$ at $1400 \mathrm{rpm}$ and spread on the preheated glass slides. Again, the slides were stacked, which allowed for an even spreading of the polymer between the slides, and incubated at $80{ }^{\circ} \mathrm{C}$ for at least $4 \mathrm{~h}$. The slides were separated while still hot and thoroughly rinsed with water. After drying in a nitrogen stream, the slides were stored at room temperature and used for up to four weeks. Before experiments, small pieces of biontinylated cover glass were incubated with neutravidin $(0.1 \mathrm{mg} / \mathrm{ml}, 10 \mathrm{~min})$ in a small petri dish, rinsed with buffer and subsequently mounted in the conical measurement chamber.

Before adhesion, the GUV suspension was cleared from small lipid debris by utilizing the density gradient between sucrose solution and PBS buffer. $100 \mu \mathrm{l}$ of GUVs containing $0.1 \mathrm{~mol} \%$ DSPE-PEG(2000)-Biotin were suspended in $1 \mathrm{ml}$ PBS in a $1.5 \mathrm{ml}$ microcentrifuge tube and allowed to sediment for $15 \mathrm{~min}$. From the bottom of the sedimentation tube $50 \mu \mathrm{l}$ of GUV suspension was pipetted into the measurement chamber containing $1 \mathrm{ml}$ of PBS. After 10 min of incubation, the buffer was gently exchanged several times with a pipette. 


\subsubsection{Permeabilization and Partitioning assay}

The adhered GUVs were used to measure the influx of fluorescently labeled lipid sidechains (LSCs) into GUVs over time and the partitioning of the LSCs into the bilayer. Control experiments were conducted with pyranine and AF488 linked to acetic acid. Before the addition of fluorescent dye, nearly all buffer was slowly removed until only the small cavity formed by the silicone ring remained covered (approx. $10 \mu \mathrm{l}$ ). Then a specified amount of PBS buffer was added (100-200 $\mathrm{ll})$. Directly underneath the microscope, an equal amount of fluorescent dye in PBS was added and gently mixed in with a pipette. The concentration of the dye solution was usually $1.6 \mu \mathrm{M}$, resulting in an end concentration of $0.8 \mu \mathrm{M}$. After dye addition, the adhered GUVs were imaged via CLSM over time with a 20x water immersion objective (W Plan Apochromat, NA 1.0, Zeiss). The fluorescent marker was excited at $\lambda_{\mathrm{ex}}=488 \mathrm{~nm}$ and emission was detected at $\lambda_{\mathrm{em}}=505-700 \mathrm{~nm}$. The focal plane was placed 5 to $10 \mu \mathrm{m}$ above the substrate, allowing the observation of adhered vesicles in a wide range of sizes.

For the detailed imaging of individual GUVs, an objective with a higher magnification was used (W Plan Apochromat 63x, NA 1.0, Zeiss). The focal plane was adjusted for each micrograph to the equatorial plane of the targeted vesicle in order to consistently measure the membrane fluorescence. The curvature of GUVs is low enough that the membrane can be treated here as a vertical plane perpendicular to the image plane. The imaging of a section through a vertical membrane rather than through the horizontal cap of the vesicle, results in a higher contrast between membrane and background due to the low resolution in the zdirection (see Figure 3.10A). Additionally, small deviations from the ideal observation plane in z-direction have a much bigger effect on the signal when imaging horizontal membranes. On the other hand, the finite curvature of the vesicles introduces an asymmetric signal, as light from planes above and below the equatorial plane is scattered into the image, which affects the signal only on the inside of the vesicle. For the observation of weakly accumulating substances, however, the advantage of a high contrast signal directly at the membrane outweighs the disadvantage of more scattered light further inside the vesicle. 

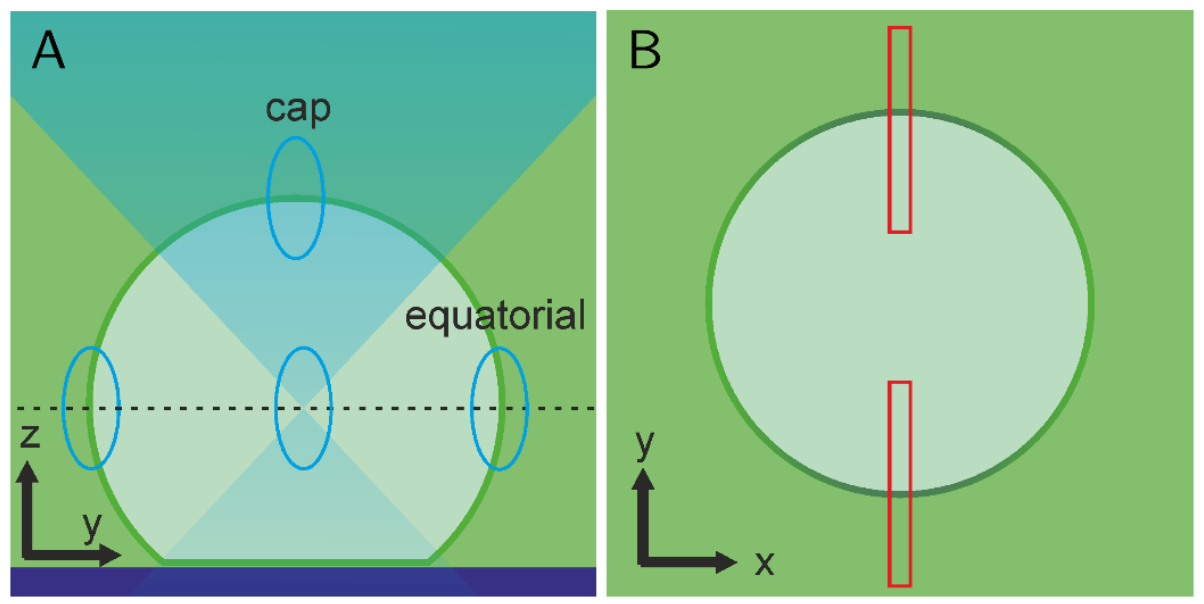

Figure 3.10 Schematic view of an adhered GUV. A: side view, showing the z-position of the equatorial focal plane (dashed line) and the asymmetry of the PSF (blue ellipses, not to scale). B: Cross section of the GUV at the focal plane. The membrane and background fluorescence are read out from vertical

regions of interest at the locations of brightest membrane fluorescence.

The membrane's fluorescence intensity was observed to be dependent on its orientation relative to the polarization plane of the laser illumination. This anisotropy is a well-known phenomenon for membrane bound fluorescent dyes ${ }^{[75]}$ and was observed for all lipid side chain (LSC) conjugates. As the weaker signal of the membrane segments parallel to the yaxis (left and right side of the GUV) was below the detection limit in some cases, only the stronger membrane signal parallel to the x-axis (top and bottom) was evaluated to quantify the membrane intensity. (see Figure 3.10B)

\subsubsection{Data analysis}

The influx of dye into adhered GUVs over time was analyzed with a custom script in MATLAB (version R2017b). A time series of micrographs was loaded, illumination-corrected with a bright image, and displayed. A suitable frame of the series was selected and used to automatically place regions of interest (ROIs) inside each vesicle via the inbuilt functions "imbinarize", "watershed", and "regionprops". The ROIs were visually inspected and corrected where necessary; mobile vesicles were excluded from the evaluation. Several reference ROIs of the free solution were manually selected. For each frame of the time series, the mean fluorescence intensity of each ROI was divided by the mean intensity of the respective reference ROIs, giving the fill ratio for each vesicle over time. 
The dye partitioning between aqueous solution and lipid bilayer was evaluated from detailed micrographs of the equatorial plane of individual adhered GUVs. The ratio of membrane and solution intensity was analyzed with a custom MATLAB script. In brief, the rough position of the vesicle was manually selected and subsequently refined by fitting the top and bottom portions of the vesicle membrane to a quadratic function. Then thin vertical ROIs were placed on the maxima of these fits (see Figure 3.10B). This method allows the repeatable selection of the GUV portions parallel to the x-axis, even for distorted shapes.

An intensity curve from the outside to the inside of the vesicle was obtained from the horizontal mean of the thin ROI along the y-axis (Figure 3.11). This averaging introduces a small distortion of the membrane peak due to the vesicle curvature. Still, it proved to be necessary to evaluate low membrane intensities, as the signal to noise ratio of individual linescans was too low to allow any analysis.
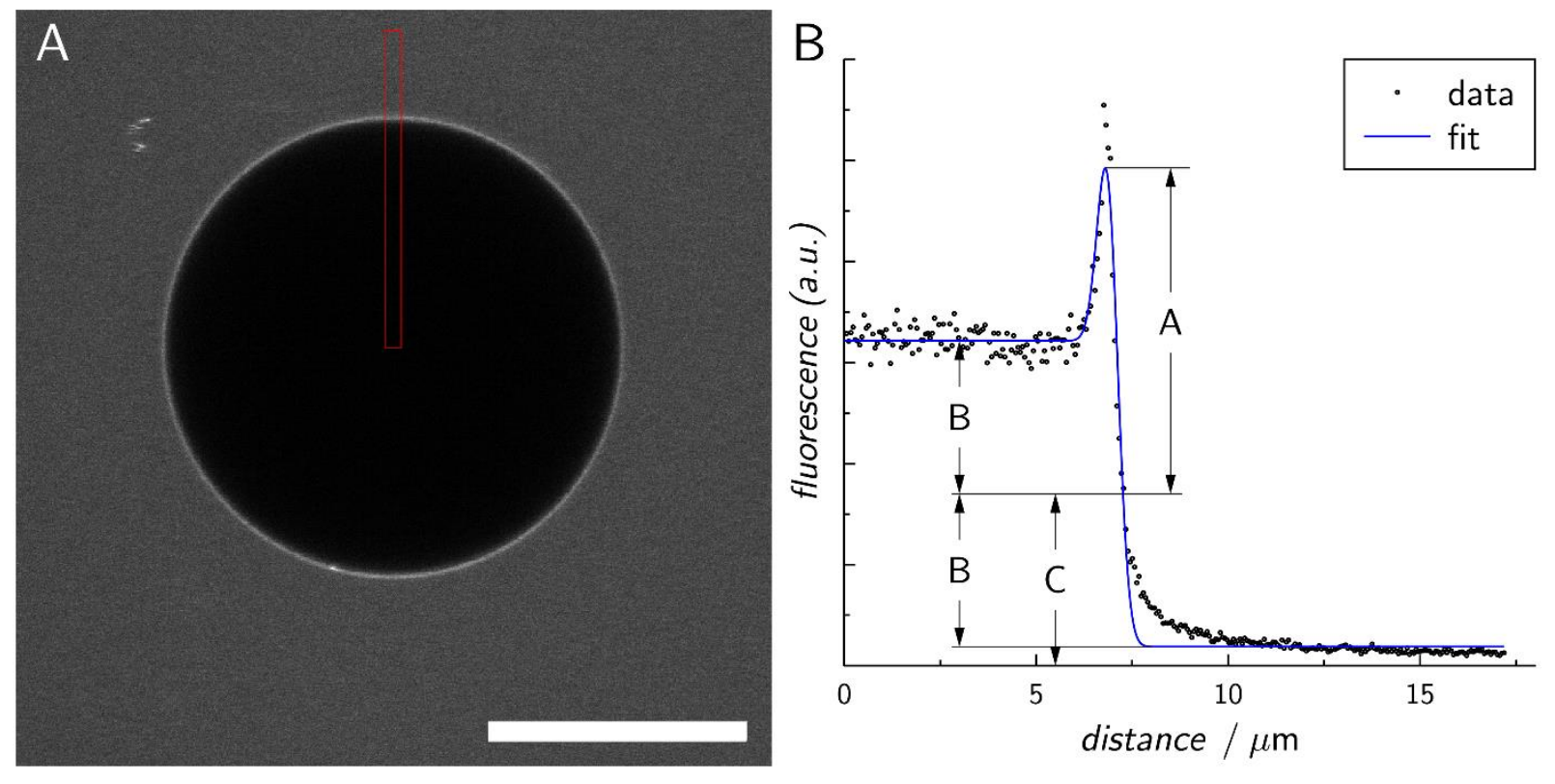

Figure 3.11: Example of a typical measurement and evaluation. A: Fluorescence micrograph of a POPC GUV in buffer containing $0.8 \mu \mathrm{M}$ w-hydroxyguanidino-LSC. The red rectangle marks the region of interest (ROI) for the data analysis. Scale bar $20 \mu \mathrm{m}$. B: Horizontal mean intensity over vertical distance for the ROI shown in A. The fitted line is calculated from equation ( 1 ) with the fitting parameters indicated by $\mathrm{A}, \mathrm{B}$, and $\mathrm{C}$. 


\section{Materials and Methods}

The averaged intensity curve was fitted with the sum of a Gaussian distribution and an error function describing the membrane intensity and the change in background intensity, respectively.

$$
f i t(x)=A * e^{-\left(\frac{x-x_{0}}{\sigma}\right)^{2}}+B * \operatorname{erf}\left(\frac{x-x_{0}}{\sigma}\right)+C
$$

This fit converged reliably under the assumptions of equal position of Gaussian peak and inflection point of the error function, as well as an equal x-scaling factor $\sigma$ for both functions. (see Figure $3.11 \mathrm{~B})$. At the position of the membrane $\left(\mathrm{x}-\mathrm{x}_{0}\right)$, the sum of the Gaussian function and the function describing the background intensity directly corresponds to $I_{\text {measured }}$ in the definition of the partition coefficient $P_{a p p}$ in equation ( 8 ) (see chapter 4.1.2). The local background intensity $I_{\text {background }}$ is given by the offset factor $\mathrm{C}$ and the membrane intensity $I_{\text {membrane }}$ is expressed in the amplitude A of the Gaussian term. The partition coefficient $P_{a p p}$ is calculated from the offset factor and Gaussian amplitude as the quotient A/C. The outer and inner solution intensities are given by $(\mathrm{C}+\mathrm{B})$ and $(\mathrm{C}-\mathrm{B})$, respectively.

The asymmetry in the raw data in the form of stray light on the inside of the vesicle was not modeled correctly by the error function, however. This led to a slight overestimation of the inner solution intensity in small vesicles and consequentially an unwarranted influence of GUV size on the measured partitioning. The addition of a third term, replacing the right part of the error function by an exponential decrease, solved this issue (see Figure 3.12). The correction term is multiplied by the Heaviside step function $H(\mathrm{x})(0$ for $\mathrm{x}<0,1$ for $\mathrm{x} \geq 0)$, thus only acts on the right side of the peak (inside the GUV). There, the error function is removed from the fit and the decrease in signal is modeled by an exponential instead (equation ( 2 )). The method of first fitting the data with equation ( 1 ) and subsequently refining the fit parameters by modeling the exponential decrease of stray light with equation ( 2 ) proofed most successful for the evaluation of noisy data.

$$
f i t_{c o r r}(x)=f i t(x)+H\left(x-x_{0}\right) *\left(B *\left(1-\operatorname{erf}\left(\frac{x-x_{0}}{\sigma}\right)\right)-B * e^{-\frac{x-x_{0}}{\tau}}\right)
$$



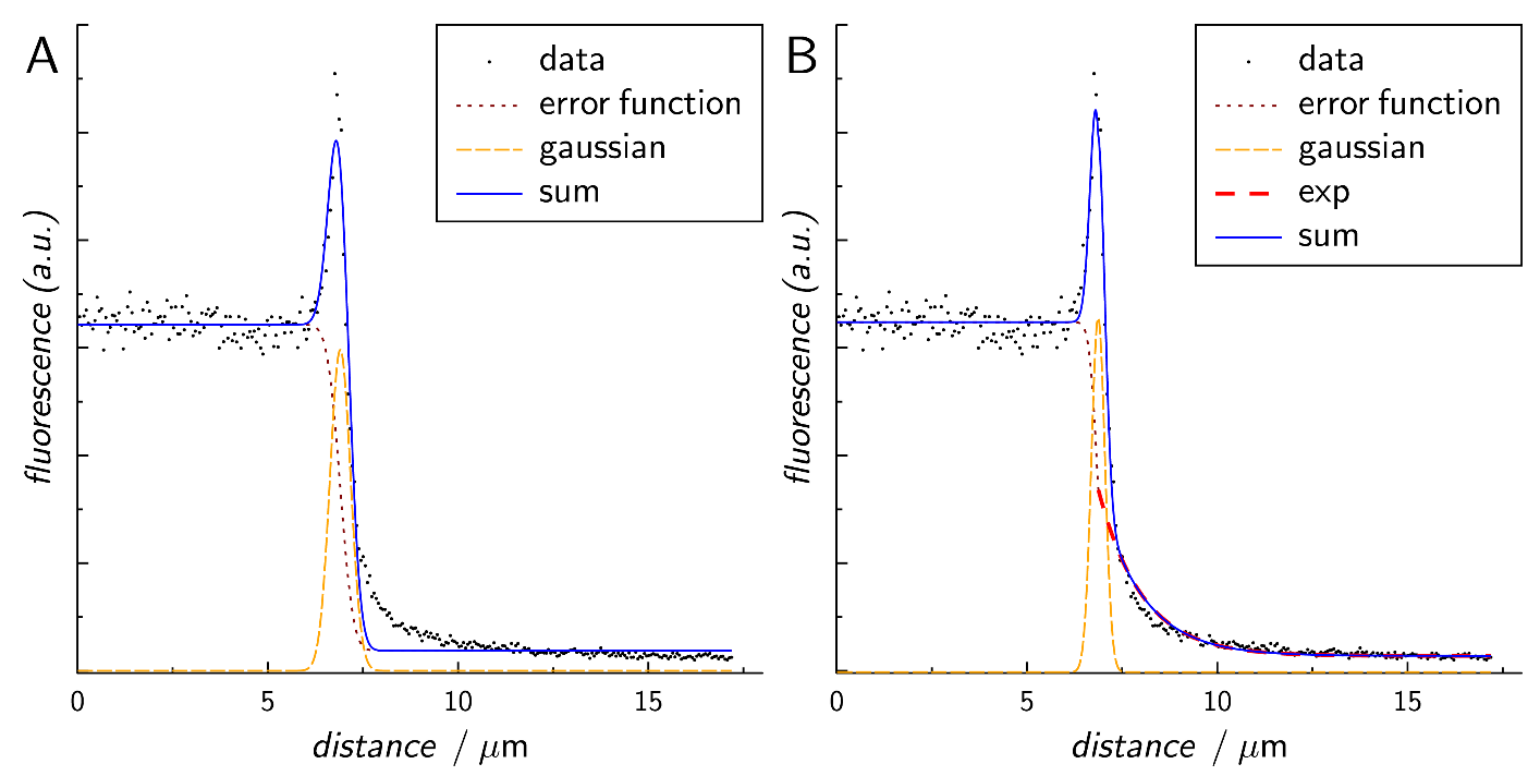

Figure 3.12: Comparison of the data fits after A: equation ( 1 ) and B: equation ( 2 ). Horizontal mean intensity over vertical distance for the data shown in Figure 3.11.

\subsection{Vesicle Assays}

All vesicle assays were performed with a Jasco FP-6500 spectrofluorometer (JASCO Germany GmbH, Gross-Umstadt, Germany) at $20{ }^{\circ} \mathrm{C}$. If not stated otherwise, excitation and emission bandwidth was set to $5 \mathrm{~nm}$. Samples were measured in a $1.5 \mathrm{ml}$ quartz cuvette and stirred with a magnetic stir-bar. Peptide was added with a $10 \mu l$ glass syringe through a septum.

Lugdunin, methyl-lugdunin, and gramicidin S were synthesized and kindly provided by the Grond group from the University of Tübingen.

\subsubsection{Permeabilization assay with carboxyfluorescein dequenching}

The membrane integrity of vesicles can be monitored by the efflux of membrane impermeable dyes when they are employed at self-quenching concentrations. Upon leaving vesicles due to lysis or pore formation, the dye is diluted and the fluorescence intensity of the sample increases. Widely used dyes for this method are calcein and carboxyfluorescein $(\mathrm{CF}) .{ }^{[76,77]}$ 


\section{Materials and Methods}

LUVs composed of POPC and filled with $100 \mu \mathrm{M}$ CF (in $100 \mathrm{mM} \mathrm{KCl,} 20 \mathrm{mM}$ HEPES, pH 7.4) were prepared via extrusion. The external buffer was exchanged with equal-osmolar dyefree buffer (275 mM KCl, $20 \mathrm{mM} \mathrm{HEPES,} \mathrm{pH} \mathrm{7.4)} \mathrm{by} \mathrm{gel} \mathrm{filtration.} \mathrm{Depending} \mathrm{on} \mathrm{the}$ determined phosphate content, 30-50 $\mu$ of vesicle suspension was diluted to $800 \mu \mathrm{L}$ to give a final lipid concentration of $50 \mu \mathrm{M}$. Dye leakage was monitored as an increase in fluorescence at $\lambda_{\text {ex }}=480 \mathrm{~nm}$ and $\lambda_{\mathrm{em}}=520 \mathrm{~nm}$. Peptide was added in a volume of $8 \mu \mathrm{L}$ as a solution in DMSO (final concentration 1 or $5 \mu \mathrm{M}$ ). After $15 \mathrm{~min}$ the vesicles were lysed by addition of $10 \mu \mathrm{L} \mathrm{10 \%} \mathrm{(w/v)} \mathrm{Triton} \mathrm{X-100} \mathrm{(Octoxinol} \mathrm{9).} \mathrm{The} \mathrm{fluorescence} \mathrm{signal} \mathrm{was} \mathrm{normalized} \mathrm{to} \mathrm{the}$ intensities directly before addition of peptide and after lysis.

\subsubsection{Ion transport assays}

The measurement of ion transport across lipid membranes is a key step in the characterization of many different classes of substances, from small proton carriers to large assemblies of protein channels. ${ }^{[78]}$ The range of different measurement techniques is equally diverse and has been steadily advanced during the last decades. The prerequisite for passive transport of any ion across a barrier is a driving force, either in the form of a concentration gradient, an electrical potential, or any combination thereof. The direct application of an electrical potential across a well-defined lipid bilayer that separates two accessible aqueous compartments has unique advantages and is essential for many applications, e.g., single channel conductance measurements via the patch-clamp method. ${ }^{[79,80]}$ In many cases, though, bulk measurements with liposomes offer a versatile and safe route for investigating the basic properties of membrane interacting substances. When using liposomes, the electrochemical gradient needed for transport is given by an imbalance between the interior and exterior concentration of either the ion of interest or a secondary ion, which can convert a concentration gradient into an electrical potential by traversing the lipid bilayer. ${ }^{[81]}$ Under certain conditions the transport can be directly detected via ion-selective electrodes, ${ }^{[82]}$ but usually optical techniques are preferred. 


\section{ACMA acidification Assay}

The lipophilic fluorophore 9-Amino-6-chloro-2-methoxyacridin (ACMA) is a widely used probe to measure proton flux. ${ }^{[78,83,84]}$ The neutral molecule is membrane permeable and is in equilibrium with the membrane impermeable protonated form. When vesicles are acidified, unprotonated ACMA is removed from the equilibrium, leading to an accumulation of dye inside the vesicles. As the fluorescence of ACMA is quenched upon protonation, dye

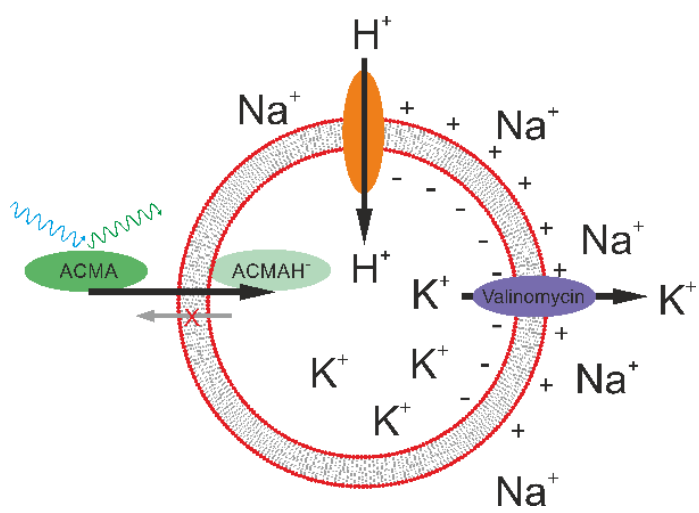

Figure 3.13: Schematic of the ACMA assay. accumulation in acidified vesicles leads to an overall decrease of fluorescence intensity. When combined with a negative electrochemical gradient across the bilayer, this enables the investigation of the permeability for protons. The gradient can be generated with the efflux of $\mathrm{K}^{+-}$ ions, mediated by the potassium ionophore valinomycin.

Small unilamellar vesicles were prepared via sonication in potassium rich buffer $(150 \mathrm{mM}$ $\mathrm{KCl}, 7.5 \mathrm{mM} \mathrm{NaCl}, 20 \mathrm{mM}$ HEPES, $\mathrm{pH} 7$ ) at a concentration of $2 \mathrm{mg} / \mathrm{ml}$. For the measurements, $30 \mu \mathrm{l}$ of vesicle suspension was mixed with $744 \mu \mathrm{l}$ sodium-rich buffer $(150 \mathrm{mM}$ $\mathrm{NaCl}, 7.5 \mathrm{mM} \mathrm{KCl}, 20 \mathrm{mM}$ HEPES, pH7) to give a final lipid concentration of $100 \mu \mathrm{M}$. Fluorescence was monitored at $\lambda_{e x}=410 \mathrm{~nm}, \lambda_{\mathrm{em}}=490 \mathrm{~nm}$ and $8 \mu \mathrm{l}$ of $0.2 \mathrm{mM}$ ACMA in EtOH was added. After a stable baseline was observed, $10 \mu \mathrm{l}$ valinomycin (0.9 $\mu \mathrm{M}$ in DMSO) was added to generate the electrochemical gradient. When the signal stabilized again, peptide was added in a volume of $8 \mu \mathrm{L}$ as a solution in DMSO (final concentration 1 or $5 \mu \mathrm{M}$ ). After the response had reached a minimum, the protonophore CCCP was added to reach complete equilibrium. The fluorescence signal was normalized to the intensity directly before the addition of valinomycin.

Pyranine $\mathrm{pH}$ Assay

As the in-situ generation of an electrochemical gradient via potassium flux is only suitable for the investigation of exclusive proton transport, an alternative assay was employed for 


\section{Materials and Methods}

the investigation of lugdunin. When using a direct proton gradient, both proton transport and cation counter-transport can be measured with a fluorescence pH-probe. ${ }^{[85]}$ To generate a proton gradient, vesicles filled with the $\mathrm{pH}$-sensitive fluorescent dye pyranine at pH 7.4 were diluted in buffer with $\mathrm{pH} 6.4$ or 8.4 to a final lipid concentration of $50 \mu \mathrm{M}$ and a volume of 800 pl. All measurements were performed in buffer containing $100 \mathrm{mM} \mathrm{NaCl}, \mathrm{KCl}$, or $\mathrm{CsCl}$, and $5 \mathrm{mM}$

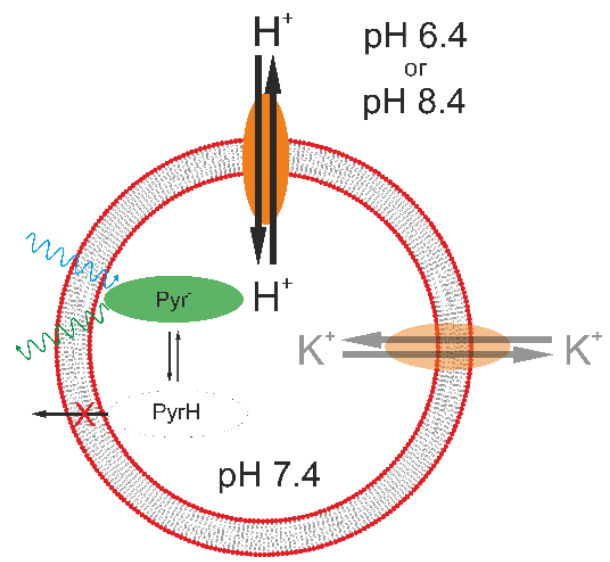

Figure 3.14: Schematic of the $\mathrm{pH}$ assay.

HEPES. The $\mathrm{pH}$ was adjusted with $\mathrm{NaOH}, \mathrm{KOH}$, or $\mathrm{Cs}_{2} \mathrm{CO}_{3}$, respectively. Pyranine fluorescence was monitored at $\lambda_{\mathrm{ex}}=458 \mathrm{~nm}$ and $\lambda_{\mathrm{em}}=512 \mathrm{~nm}$. After acquisition of a baseline for $250 \mathrm{~s}, 8 \mu \mathrm{l}$ of a 2.5 to $500 \mu \mathrm{M}$ solution of peptide in DMSO was added. At the end of each measurement, the vesicles were lysed by addition of TX-100. Artifacts due to incomplete lysis were found when adding only $10 \mu \mathrm{l}$ TX-100 (1.9 mM), although this concentration lies well above the critical micelle concentration of $0.22 \mathrm{mM} .^{[86]}$ These artifacts were not observed with $16 \mu \mathrm{l} 10 \%$ (w/v) TX-100, which was thus adopted as the standard concentration for vesicle lysis. The fluorescence intensity was normalized to range from 0 to 1 , corresponding to the values directly before peptide addition and after lysis.

To determine the amount of pyranine leakage, vesicles were suspended in buffer at $\mathrm{pH} 8.4$ and instead of TX-100 the membrane impermeable quencher $p$-xylene-bis-pyridinium bromide (DPX) (16 ul, $0.2 \mathrm{M}$ in DMSO) was added $300 \mathrm{~s}$ after peptide addition. The fluorescence intensity was normalized to the maximum reached after addition of lugdunin at a P/L ratio of 1:10. A titration of free pyranine with DPX showed a quenching efficiency of 0.94 for a concentration of $4 \mathrm{mM} \mathrm{DPX}$.

To measure proton flux independently from peptide insertion, pyranine filled vesicles were diluted in buffer of equal $\mathrm{pH}(7.4)$ to a final lipid concentration of $50 \mu \mathrm{M}$ and a volume of $800 \mu \mathrm{l}$. Then $8 \mu \mathrm{l} 5 \mu \mathrm{M}$ peptide was added, either as solution in DMSO or isopropanol. In control experiments, $8 \mu \mathrm{l}$ solvent were added. Fluorescence intensity was recorded at $\lambda_{\mathrm{ex}}=458 \mathrm{~nm}$ and $\lambda_{\mathrm{em}}=512 \mathrm{~nm}$. After $300 \mathrm{~s}, 10 \mathrm{ul} 0.125 \mathrm{M} \mathrm{HCl}$ was added to the cuvette, followed by $20 \mu \mathrm{l}$ TX-100. As a positive control, vesicles containing lugdunin in the bilayer 
were prepared. To attain theses vesicles, lugdunin in isopropanol was added to the lipid solution in chloroform before the lipid film preparation (see chapter 3.1.2). The vesicles were suspended in buffer as before and acidified without further incubation with peptide. 


\section{Materials and Methods}

\subsection{Pore-spanning membranes on porous aluminium oxide}

The goal of the lab-on-a-chip approach pursued in this thesis is the transfer of membrane model systems using vesicles in solution to a 2D model system: an adhered lipid bilayer on a porous substrate, forming pore spanning membranes. These have the distinct advantage over completely supported bilayers, that both sides of the membrane are adjacent to the aqueous phase.

The AAO layer obtained from anodization of aluminium is comprised of an oxide matrix containing cylindrical pores in a hexagonal pattern. At the interface to the metal, these pores are closed by a dense oxide layer which remains intact even after removing the metal substrate by etching. When covered by pore-spanning membranes, attoliter-sized compartments are formed, allowing the precise monitoring of influx or efflux of fluorescent dyes through the lipid membrane. ${ }^{[28]}$

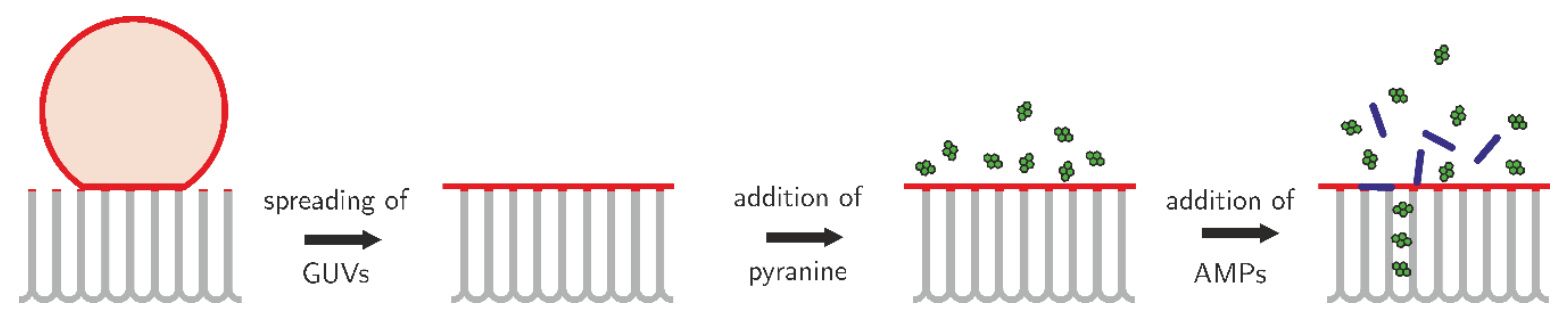

Figure 3.15: Schematic illustration of the permeabilization assay with PSMs on AAO. Not to scale.

The advantage of using self-organized anodic oxide films is the ease with which the basic geometric features, pore diameter and depth, can be tuned directly in the lab. A wide range of pore diameters is available for anodic aluminium oxide, from less than $25 \mathrm{~nm}$ up to several hundred nanometers. ${ }^{[87][88]}$ The thickness of the porous substrate can be controlled by anodization time from a few $\mu \mathrm{m}$ to over 100.[89]

\subsubsection{Preparation of porous alumina}

Porous alumina was prepared in a two-step anodization process (see Figure 3.16) from aluminium sheets $0.5 \times 20 \times 20 \mathrm{~mm}^{3}$ in size $(99.999 \%$, Goodfellow Cambridge Ltd., United Kingdom). These chips were annealed in nitrogen atmosphere $\left(500{ }^{\circ} \mathrm{C}, 12 \mathrm{~h}\right)$ and 
electrochemically polished. For this, sets of four chips were clamped between a copper baseplate and a custom-built Teflon beaker with four openings in the bottom, sealed by $16 \mathrm{~mm}$ O-rings. The beaker was filled with a mixture of $\mathrm{H}_{2} \mathrm{SO}_{4}$ (conc.) $/ \mathrm{H}_{3} \mathrm{PO}_{4}(85$ wt $\%) / \mathrm{H}_{2} \mathrm{O}(1: 1: 1$ $\mathrm{v} / \mathrm{v} / \mathrm{v})$. It was heated to $65{ }^{\circ} \mathrm{C}$ on a hot-plate and a voltage of $25 \mathrm{~V}$ was applied between the base plate (positive) and a platinum wire grid in the polishing solution. After 15 min, the solution was replaced and polishing was continued for further $15 \mathrm{~min}$. The polished chips were removed from the beaker, rinsed, and etched in 5 wt $\% \mathrm{H}_{3} \mathrm{PO}_{4}$ for approx. $2 \mathrm{~h}$ until no residual oxide was observed during drying in a nitrogen stream.

For the anodization, the thoroughly rinsed chips were again clamped between a copper baseplate and a beaker, with the polished areas carefully aligned to the openings. To avoid excessive gas bubble adhesion in the vicinity of the chip, beakers made from PVC instead of Teflon were used in this step. The beaker was placed on a water-cooled Peltier element set to $2.5^{\circ} \mathrm{C}$ and filled with precooled $0.3 \mathrm{M}$ oxalic acid. The solution was stirred by a motorized glass stir bar mounted in a thermally isolated sleeve that was placed around the beaker. After $30 \mathrm{~min}$, a voltage of $40 \mathrm{~V}$ was applied between the base plate and a platinum wire grid inside the solution. After $3 \mathrm{~h}$, the anodization was stopped, and the oxide layer was removed by etching in $5 \mathrm{wt} \% \mathrm{H}_{3} \mathrm{PO}_{4}$ for approx. $3 \mathrm{~h}$. Again, complete oxide removal was judged by eye, as the etched AAO appears as a milky white residue during drying. Subsequently, the pre-structured aluminium chips were anodized again under identical conditions for 1.5-6 h, depending on the required AAO thickness.

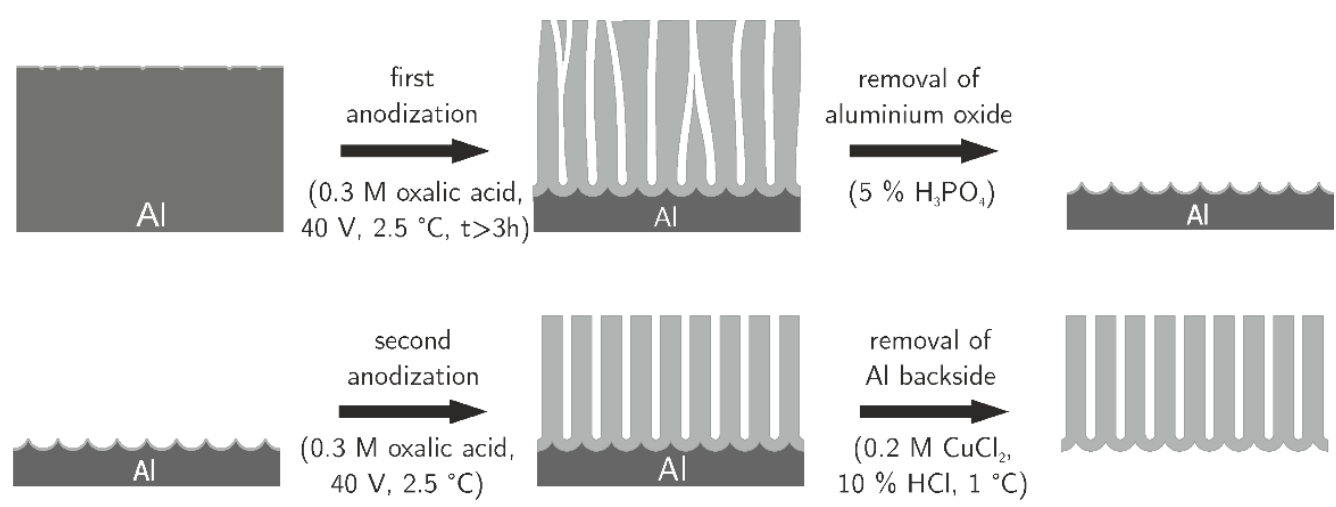

Figure 3.16: Schematic depiction of the 2-step anodization process and metal substrate removal. 


\section{Materials and Methods}

To obtain a freestanding AAO substrate, the metal beneath the oxide layer was removed from the backside by etching. Before etching, both sides of a chip were coated with a thin layer of photoresist (AZ4562, Microchemicals GmbH, Germany, 3:1 in acetone). This measure protects the oxide layer and surrounding metal from unwanted etching in case of small leaks. After drying for at least 12 h, individual chips were clamped oxide-side down between a copper baseplate and a beaker with a single opening at the bottom. To keep the chip from sticking to the baseplate and to provide a bright background for the observation of the etching progress, a baseplate covered with a Teflon coated adhesive foil was used. Additionally, a few drops of water were placed in the gap between baseplate and AAO chip, which provides mechanical stability to the fragile AAO layer at the end of the etching process. The photoresist was exposed through the hole in the beaker with a collimated UVLED (365 nm, $1 \mathrm{~W}, 60 \mathrm{~s}$ ) and was removed with $0.2 \mathrm{M} \mathrm{NaOH}$. Subsequently, $50 \mathrm{ml}$ of etching solution $\left(0.2 \mathrm{M} \mathrm{CuCl}_{2}\right.$ in $\left.10 \% \mathrm{HCl}\right)$ was added to the beaker, followed by $3 \mathrm{ml}$ of $30 \% \mathrm{H}_{2} \mathrm{O}_{2}$. The solution was agitated by pipetting until the reaction accelerated, signified by the onset of strong hydrogen evolution. The reaction was continued for $3 \mathrm{~min}$ at this high rate, before placing the beaker onto a cooling plate held at $5^{\circ} \mathrm{C}$. The etching was stopped when all metal was removed from the central area, leaving a ring of approx. $1 \mathrm{~mm}$ at the edges to avoid a breakthrough.

The chip was removed from the beaker, rinsed on the etched backside with concentrated $\mathrm{HNO}_{3}$ to remove copper residues, and rinsed thoroughly with water. The photoresist was removed with acetone and the chip was glued to a plastic ring using nail polish, thus forming a small chamber on the etched backside (see Figure 3.17). All aluminium exposed in the chamber was carefully covered with nail polish, ensuring electrical isolation. The closed pores were then opened by etching with $5 \% \mathrm{H}_{3} \mathrm{PO}_{4}$ for $1-3 \mathrm{~h}$. The process was monitored by performing electrochemical

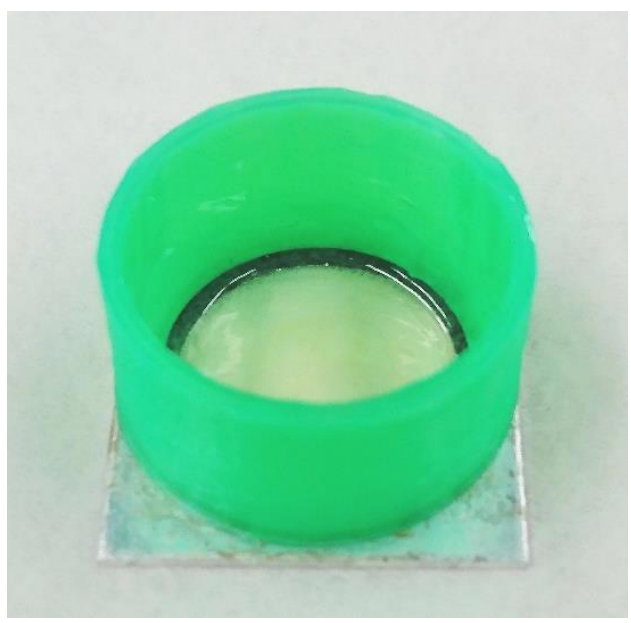

Figure 3.17: AAO chip with attached chamber for etching and electrodeposition. impedance spectroscopy in regular intervals. The 
AAO was contacted with a copper plate and a few drops of saturated HEPES solution ( $\mathrm{pH} 7$ ) on the underside and a platinum electrode in the acid filled chamber (2-electrode setup). Pore opening was observed as a gradual decrease in the absolute value of impedance at $1000 \mathrm{~Hz}$ over the course of approx. 5 min. After pore opening, the sample was submersed in $5 \% \mathrm{H}_{3} \mathrm{PO}_{4}$ for $20-50$ min to widen the pores to the required diameter. The time needed to obtain standard $60 \mathrm{~nm}$ pores was $40 \mathrm{~min}$. The AAO was rinsed with water and dried for at least $12 \mathrm{~h}$ in ambient air before further processing.

\subsubsection{Electrodeposition of Ag-Wires}

The incorporation of silver electrodes into the porous substrate was performed via electrodeposition. The Ag-wires were introduced into the top side of the AAO substrate, leaving the opened pore bottoms as the surface for later functionalization and bilayer spreading.

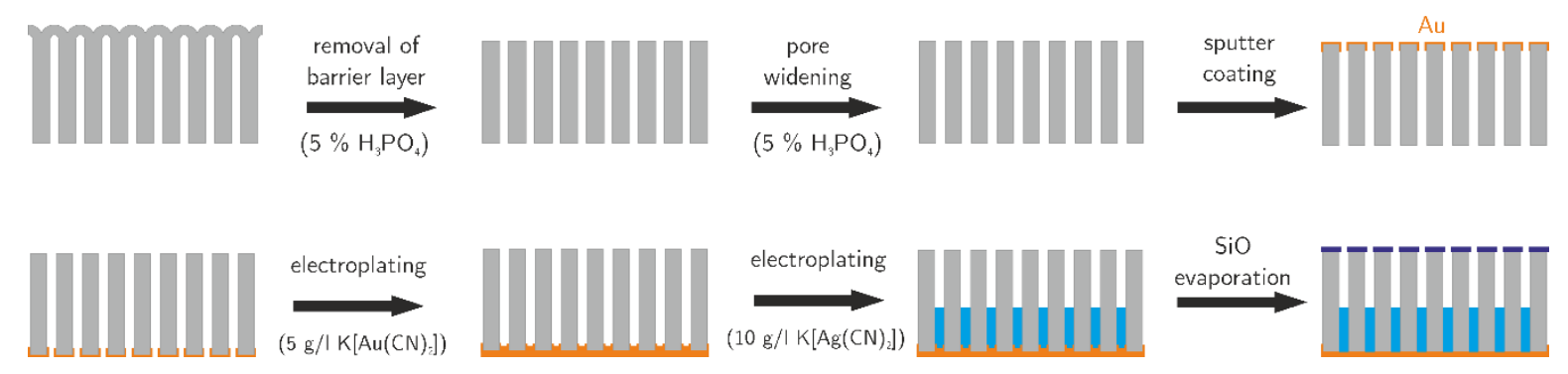

Figure 3.18: Schematic overview of the processing steps performed to obtain an AAO substrate with embedded silver electrodes.

In a first step, the thoroughly dried AAO chips were electrically contacted by sputtering $50 \mathrm{~nm}$ gold onto the top surface (40 mA, $0.1 \mathrm{mbar}$, Sputter Coater 108auto, Cressington Scientific Instruments, United Kingdom). The sputtered gold contact was thickened to the point of complete sealing of the pores by electrodeposition of gold. For this step, the chip was flipped and the small chamber formed by the attached ring was filled with gold electrolyte $(5 \mathrm{~g} / 1$

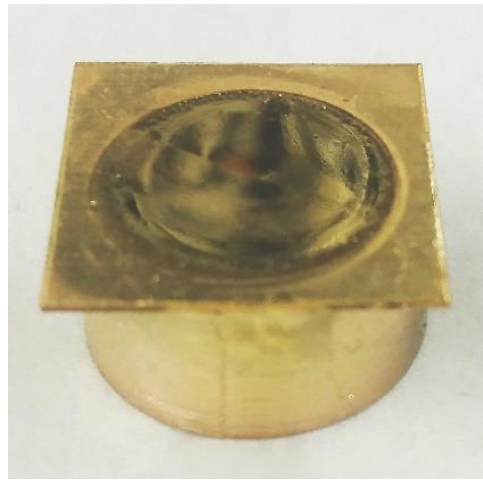

Figure 3.19 AAO chip after gold sputtering $\left.\mathrm{K}\left[\mathrm{Au}(\mathrm{CN})_{2}\right], \quad 5 \mathrm{~g} / 1 \quad \mathrm{NH}_{4} \mathrm{Cl}\right)$. The aluminium plate was 


\section{Materials and Methods}

electrically connected via an alligator clip and a small loop of platinum wire was placed into the electrolyte as counter electrode. Both electrodes were connected to a potentiostat (CompactStat, Ivium Technologies B.V., The Netherlands) in a 2-electrode setup. Gold deposition was performed galvanostatically at $-1 \mathrm{~mA} / \mathrm{cm}^{2}$ until the color of the layer changed from black to gold when seen through the AAO.

For the subsequent silver deposition, the chamber was rinsed and filled with a silver electrolyte. Both cyanide-free $\left(50 \mathrm{mM} \mathrm{AgNO}_{3}, 0.5 \mathrm{M} \mathrm{Na}_{2} \mathrm{~S}_{2} \mathrm{O}_{3}\right)$ and classic $\left(10 \mathrm{~g} / \mathrm{K}\left[\mathrm{AgCN}_{2}\right]\right.$ $5 \mathrm{~g} / \mathrm{l} \mathrm{KCN}$ ) silver electrolytes were used with no discernable differences except for the shelflife of the electrolyte. The electrolyte was contacted by two silver wires as counter- and reference-electrode, and $\mathrm{Ag}$ was deposited at $-1 \mathrm{~mA} / \mathrm{cm}^{3}$ in a 3-electrode setup. Continuous current and pulsed (10\% duty cycle) deposition produced comparable results. The height of the resulting wire array was controlled by monitoring the amount of charge deposited, multiplied by the coulomb efficiency of the process (approx. 0.5). The chip was removed from the plastic ring by soaking in acetone/isopropanol (1:1) for several hours, followed by subsequent rinsing in acetone, ethanol, $5 \% \mathrm{H}_{3} \mathrm{PO}_{4}$, and water.

\subsubsection{Orthogonal Functionalization}

A prerequisite for the preparation of a stable 2D-System composed of pore-spanning lipid bilayers is the appropriate functionalization of the substrate. Although the spreading of vesicles on porous structures with isotropic surface properties can lead to spanned pores, ${ }^{[90]}$ an orthogonal functionalization that differentiates pore rims and the pore interior simplifies the spreading and leads to well defined bilayer geometries.

On native aluminium oxide, be it from anodic oxidation or other sources, no bilayer adhesion is possible at neutral $\mathrm{pH}$ values. This can be attributed to the layer of interfacial water at the oxide surface, which is more tightly bound than on $\mathrm{SiO}_{2}$ surfaces. Without additional forces, e.g. electrostatic attraction mediated by bivalent cations, the lipid bilayer cannot displace enough of this layer to develop sufficient adhesion. ${ }^{[1,92]}$ In the case of porous aluminium oxide this property is highly advantageous for the preparation of an orthogonal functionalization. No passivation of the pore interior is necessary, even after surface 
treatments which usually promote bilayer adhesion on oxide surfaces, such as plasma cleaning. At the same time, aluminium oxide surfaces are highly hydrophilic, which minimizes the likelihood of inadvertent trapping of air in the porous substrate. The addition of a functionalization which facilitates bilayer adhesion on the top surface of porous AAO substrates is therefore sufficient to obtain an orthogonally functionalized system that enables the spreading of pore-spanning membranes. With $\mathrm{SiO}_{2}$ and $\mathrm{Si}_{3} \mathrm{~N}_{4}$ substrates, on the other hand, special care must be taken with bare pore interiors if bilayer intrusions are to be avoided. Hydrophilization of these substrates by cleaning with oxidizing solutions or $\mathrm{O}_{2-}$ plasma can lead to lining of the pores with lipid material, irrespective of the rim functionalization.

In this thesis, three different strategies were employed to obtain orthogonally functionalized substrates. The first two strategies are based on an isotropic silanization, followed by the thermal evaporation of a gold layer on the pore rims. This gold layer serves as an orthogonal mask that permits the removal or passivation of the silane layer inside the pores and is removed prior to bilayer spreading to expose a silanol functionalized rim. The third strategy is the direct functionalization of the pore rims by thermal evaporation of $\mathrm{SiO}$, with no modifications to the pore interior.

\subsubsection{Gas phase silanization}

A well-established method for producing AAO substrates with a silanol surface functionalization on the pore rims is based on gas phase silanization with (3mercaptopropyl)triethoxysilane (MPTES) and the selective removal of the silane layer inside the pores with Ar-plasma. ${ }^{[3]}$ 

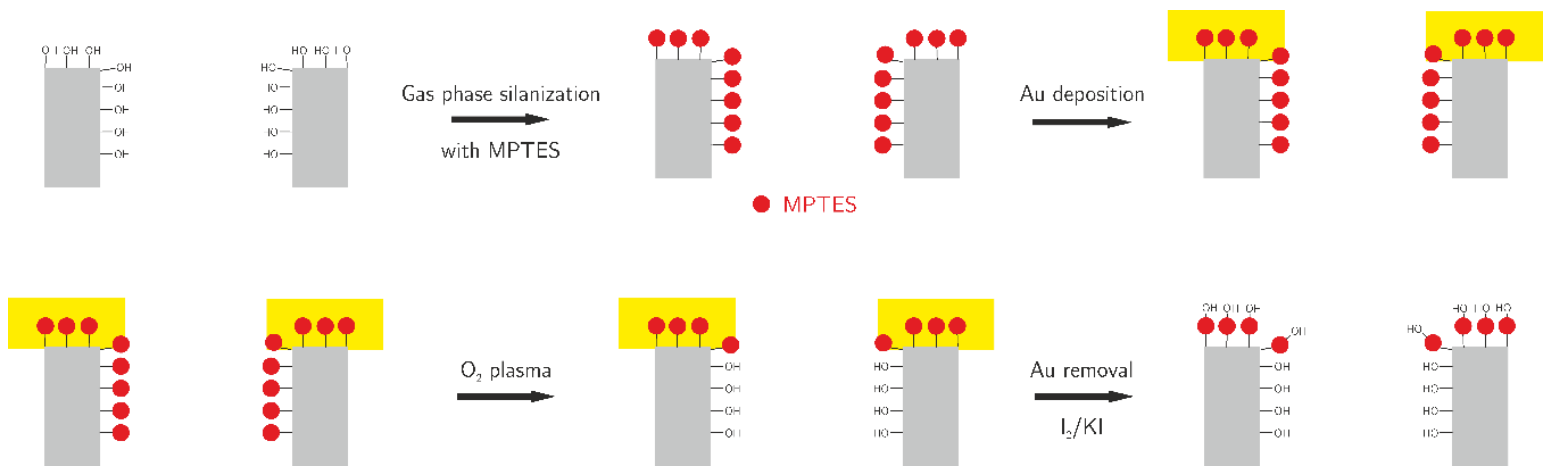

Figure 3.20: Schematic illustration of the orthogonal functionalization of AAO via gas-phase silanization with MPTES

The reasoning behind using the mercaptopropyl-silane instead of the more commonly used aminopropyl derivate APTES is the strong interaction of SH-groups with gold surfaces, improving the adhesion of the evaporated gold mask on the pore rims.

The silanization was performed by placing the substrates and a small open glass tube containing $50 \mathrm{pl}$ silane in a glass TLC chamber. The chamber then was sealed nearly gastight with polyimide adhesive tape and placed into a vacuum oven preheated to $130{ }^{\circ} \mathrm{C}$. After 10 min the oven chamber was evacuated and kept at $130{ }^{\circ} \mathrm{C}$ for $3 \mathrm{~h}$. After slowly cooling to room temperature under vacuum, the silanized AAO samples were directly transferred into the recipient of the evaporation setup (Coating System MED020, Bal-Tec, Leica, Germany). The recipient was evacuated to a residual pressure of $5^{*} 10^{-6}$ mbar and gold was evaporated from a molybdenum boat at a rate of $0.2 \mathrm{~nm} / \mathrm{s}$ to a thickness of $30 \mathrm{~nm}$ as indicated by the QCM. The gold coated substrates were stored in air at room temperature. Before use, the silane layer inside the pores was removed by treatment with Ar-plasma at 0.25 mbar, $60 \%$ power for 2 min (Zepto LF PC, Diener Electronic, Germany). The gold layer was removed with a small drop of $\mathrm{I}_{2} / \mathrm{KI}$ solution and the substrate was rinsed with water and dried in a nitrogen stream. Subsequently the freshly exposed silane layer on the pore rims was oxidized to silanol groups by treatment with $\mathrm{O}_{2}$-plasma at 0.25 mbar, $60 \%$ power for $30 \mathrm{~s}$.

\subsubsection{Fluid phase silanization and PEGylation}

The second strategy leads to a similar silanol coated rim surface as the method described above, but can also be applied on $\mathrm{Si}_{3} \mathrm{~N}_{4}$ and $\mathrm{SiO}_{2}$ substrates, as the silane layer on the pore 
interior is passivated instead of removed. The passivated silane layer must feature both hydrophilic properties, to ensure proper wetting of the pores, as well as no adhesive interaction with lipid bilayers. Polyethylenglycol (PEG) satisfies these requirements and can be easily attached to surfaces. The silane used in the first strategy was switched to APTES, which allows the coupling of its amino group with a $N$-Hydroxysuccinimide ester functionalized PEG. To speed up the preparation and also allow multiple subsequent silane layers to be deposited, silanization was performed in methanol solution instead of the gas phase. The substrates were activated with $\mathrm{O}_{2}$-plasma (60 s, $0.2 \mathrm{mbar}, 60 \%$ power), incubated in $0.1 \%$ APTES in methanol for $10 \mathrm{~min}$, rinsed with ethanol and dried in a nitrogen stream. The thickness of the silane layer is not a critical factor for orthogonality with this method, as no complete silane removal is required. AAO was silanized 2 times and oxidized in $\mathrm{O}_{2}$ plasma before evaporating a $30 \mathrm{~nm}$ gold mask onto the rims. $\mathrm{Si}_{3} \mathrm{~N}_{4}$ substrates were activated in plasma but not silanized before masking. Both types were silanized with APTES once after Au evaporation, followed by PEGylation with methoxy-PEG(1000)-NHS $(10 \mathrm{mg} / \mathrm{ml}$ in ethanol/PBS 9:1, $1 \mathrm{~h}$ at RT).

On porous but flat $\mathrm{Si}_{3} \mathrm{~N}_{4}$ substrates, the gold mask can be removed mechanically by applying an adhesive tape on the surface and slowly removing it again at a high angle. Due to the moderate adhesion of gold on oxidized $\mathrm{Si}_{3} \mathrm{~N}_{4}$, most of the gold mask is transferred to the tape and the unpassivated substrate on the rims is exposed. This method was not successful on porous AAO, where gold was removed with $\mathrm{I}_{2} / \mathrm{KI}$ solution instead.

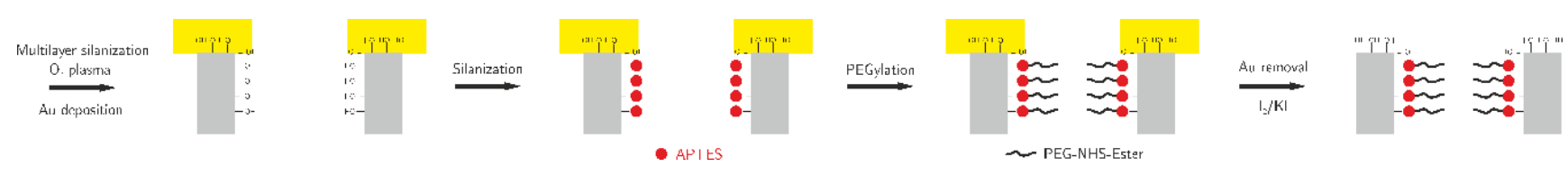

Figure 3.21: Schematic illustration of the orthogonal functionalization of AAO with APTES and PEG.

\subsubsection{Thermal evaporation of $\mathrm{SiO}$}

The final strategy to orthogonally functionalize porous substrates is the direct evaporation of silicon monoxide onto the pore rims. AAO substrates were dried for at least $12 \mathrm{~h}$, cleaned in $\mathrm{O}_{2}$-plasma $(60 \mathrm{~s}, 0.2 \mathrm{mbar}, 60 \%$ power), and directly transferred to the evaporation 


\section{Materials and Methods}

recipient. $\mathrm{SiO}$ was thermally evaporated at a residual pressure of $5^{*} 10^{-5}$ mbar from a tungsten boat at a rate of $0.3 \mathrm{~nm} / \mathrm{s}$.

The substrates were stored in air for prolonged periods of time at room temperature. Before use, they were rinsed with water, thoroughly dried in a nitrogen stream and cleaned with $\mathrm{O}_{2}$-plasma for $30 \mathrm{~s}$ at $0.2 \mathrm{mbar}$ and $60 \%$ power.
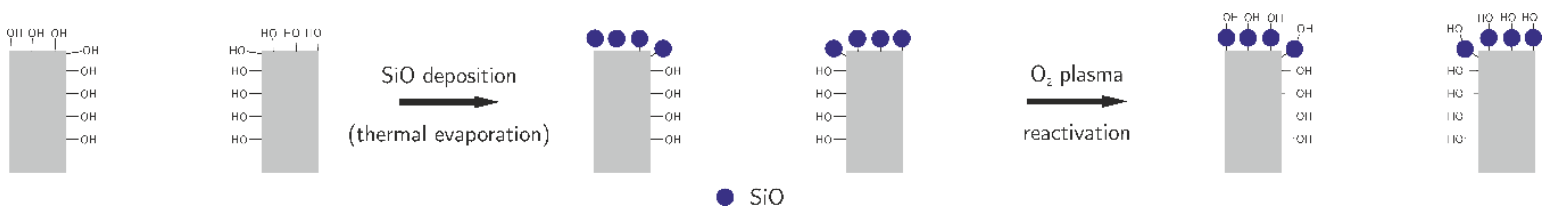

Figure 3.22: Schematic illustration of the orthogonal functionalization of AAO via thermal evaporation of $\mathrm{SiO}$.

\subsubsection{Temperature controlled spreading of phase-separated GUVs}

For the measurements with phase-separated GUVs, a way was needed to precisely control the temperature of the substrate during both the spreading of GUVs and the subsequent microscopy experiments. The requirements for the temperature stage were the ability to set a temperature between $4{ }^{\circ} \mathrm{C}$ and $55{ }^{\circ} \mathrm{C}$, a controlled rate of temperature change of at least $0.5^{\circ} \mathrm{C} / \mathrm{s}$, and a low-drift, vibration free mount. No commercial solution satisfying all these requirements was available and a custom-built microscopy stage was designed. The stage is built around a copper baseplate mounted flush on a POM insert plate. A Pt1000 chip-sensor (M222-1/3 B, Heraeus Nexensos GmbH, Kleinostheim, Germany) was glued into a slot on the top of the baseplate and connected to a precalibrated AD-converter (TEMOD-I2C-R1, $\mathrm{B}+\mathrm{B}$ Thermo-Technik GmbH, Donaueschingen, Germany). A Peltier element (generic $\left.12706,40 \mathrm{x} 40 \mathrm{~mm}^{2}, 12 \mathrm{~V} / 6 \mathrm{~A}\right)$ is sandwiched between the baseplate and a commercial CPU cooler (Thermalright ACP-200, PC-Cooling GmbH, Nortorf, Germany). A PID controller, implemented on an ATmega328p microcontroller, controls a variable voltage power supply.

The stage temperature was set to values between 0 and $55{ }^{\circ} \mathrm{C}$ with substrate temperatures closely following these values as verified by measurements with a $0.2 \mathrm{~mm}$ thermocouple. In the range of interest for the phase mixing experiments between 0 and $20{ }^{\circ} \mathrm{C}$, the temperature gradient from the substrate to the immersion objective is positive, resulting in a negative 
density gradient, and heat transfer is expected to be mostly conductive. The measured temperature deviation between substrate and stage was always $<1{ }^{\circ} \mathrm{C}$ and no significant convection was observed via microscopy. At temperatures above $30{ }^{\circ} \mathrm{C}$ the thermal layering above the substrate was visibly disturbed, as convection currents evolved. Nevertheless, during temperature ramps up to $55{ }^{\circ} \mathrm{C}$, the substrate temperature deviation remained in the range of $1{ }^{\circ} \mathrm{C}$. This relatively small deviation can be attributed to the gradual heating of the objective, keeping the heat transfer to it at a low level. Furthermore, the all-metal design and large surface area of the sample holder ensure rapid equilibration of the buffer and a distribution of the heat flux to the objective away from the substrate. The significant temperature changes of the objective required rapid manual refocusing during temperature changes, but image resolution was not affected. Thus, active control of objective temperature was deemed unnecessary for the experiments conducted.

\subsubsection{Combination of microscopy and electrochemistry}

To enable the application of a defined potential to the nanowire array incorporated into the AAO substrates while simultaneously observing the sample via CLSM, a custom measurement chamber was designed. In an iterative process, the chamber was fabricated from Polylactic acid (PLA) and Polypropylene (PP) via fused deposition on a 3D-printer (Ultimaker 2+, Ultimaker BV, Netherlands), tested, and modified where necessary. PP was chosen for its chemical inertness, but eventually discarded as the meniscus between sample and microscope objective frequently broke due to the low surface energy of PP. PLA showed good wettability and was therefore used preferentially. The chamber is conically shaped to allow insertion of a water immersion objective and minimize fluid volume. Buffer exchange is performed via two channels from the sides which are connected to peristaltic pumps. To keep the fluid level constant, both feed and drain are actively pumped. Two silver wires are bent into recesses in the chamber walls and act as counter-

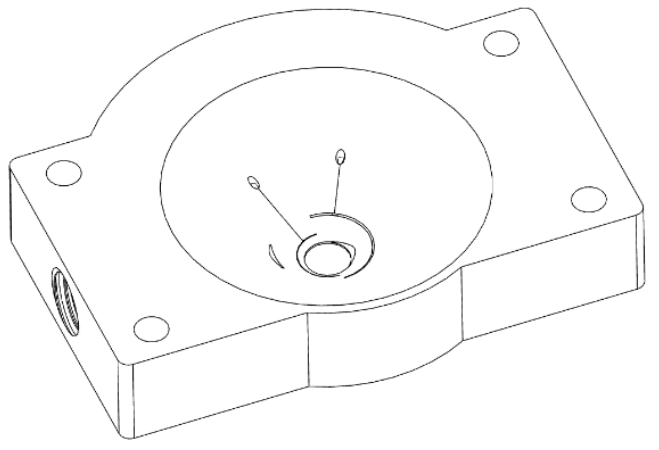

Figure 3.23: Schematic drawing of the conical chamber with embedded channels for fluidics and electrodes. 


\section{Materials and Methods}

and reference electrode. The wires leave the chamber through channels sealed with UVcuring adhesive (NOA 83H, Norland Products, USA).

The AAO chip with embedded silver wires and functionalized with $\mathrm{SiO}$ was broken into pieces approx. $4 \times 4 \mathrm{~mm}^{2}$, which were glued to gold plated copper sheets $\left(10 \mathrm{x} 10 \mathrm{~mm}^{2}\right)$ with conductive silver adhesive. After $24 \mathrm{~h}$, the copper plate and edges of the substrate were covered with UV-curing adhesive, leaving a small area of several $\mathrm{mm}^{2}$ open.

The substrate was clamped between the conical chamber and a base plate, sealed with a thin gasket cast in PDMS. A connector was screwed into the baseplate from the bottom to contact the substrate. The measurement chamber was connected to a potentiostat and clamped into place under the CLSM.
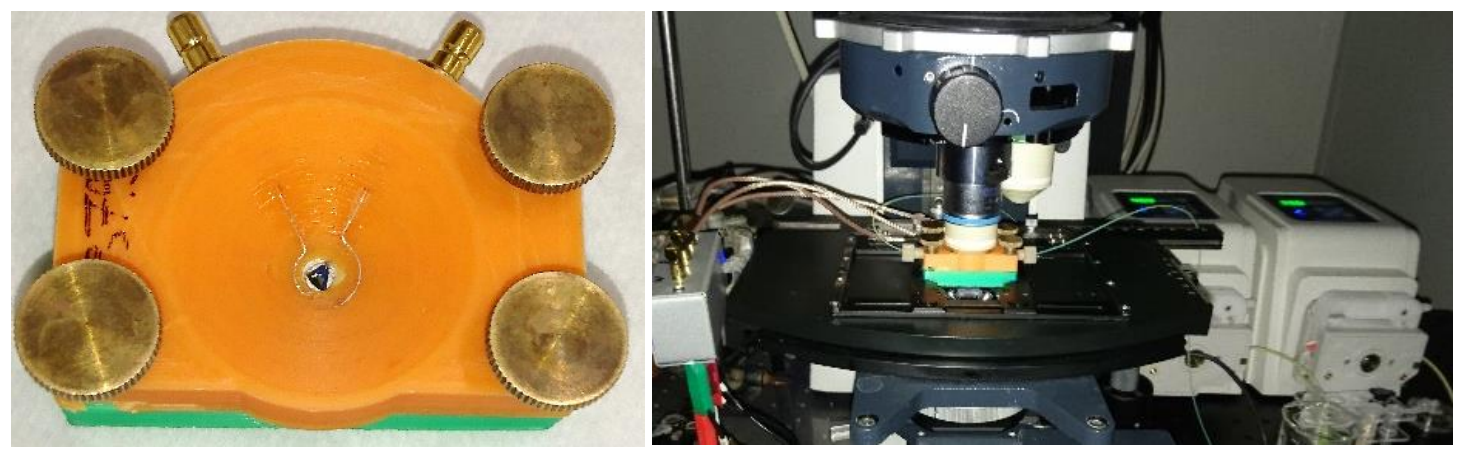

Figure 3.24: Photographs of the measurement chamber before and after integration at the CLSM

\section{Spreading of GUVs}

After substrate mounting and priming of all fluid lines with buffer, substrates were rinsed with $1 \% \mathrm{H}_{3} \mathrm{PO}_{4}$ for $10 \mathrm{~s}$ and immediately rinsed with buffer. If not stated otherwise, PBS saturated with $\mathrm{AgCl}$, freshly precipitated from $\mathrm{AgNO}_{3}$ and $\mathrm{NaCl}$, was used in all experiments involving AAO substrates with embedded nanowires. Both the reference electrode and the wire array were held in turn at a positive potential of $100 \mathrm{mV}$ for $60 \mathrm{~s}$ to form a $\mathrm{AgCl}$ covered silver surface, and potential stability was verified by monitoring the open-circuit potential. All electrochemical procedures were performed with a potentiostat in a 3-electrode configuration (CompactStat, Ivium Technologies B.V., The Netherlands).

GUV suspension obtained from electroformation was gently mixed with buffer in a ratio of 1:9 and vesicles were sedimented by centrifugation in a microcentrifuge for $5 \mathrm{~s}$ (Galaxy mini, 
VWR). For spreading of GUVs, the chamber was completely filled with buffer and $100 \mu \mathrm{l}$ of sedimented GUV suspension was added above the substrate.

For the permeabilization assay, POPC GUVs doped with $0.1 \mathrm{~mol} \%$ TexasRed-DHPE were used. After 10 min of incubation, the buffer was repeatedly exchange via pipette and pumped flow (approx. $100 \mathrm{\mu l} / \mathrm{min}$ ) was activated. Subsequently, the buffer was changed to PBS containing $1 \mathrm{mM}$ pyranine via the fluidic system. Confocal microscopy was performed on a ZEISS LSM710 Examiner equipped with a $63 \mathrm{x}$ water immersion objective (WPlan APOChromat 63, NA 1.0) and set up in a two-channel configuration with simultaneous scanning of both fluorophores $\left(\lambda_{\mathrm{ex}}=488 \mathrm{~nm}, 594 \mathrm{~nm}, \lambda_{\mathrm{em}}=500-560 \mathrm{~nm}, 600-735 \mathrm{~nm}\right) . A$ membrane patch showing good dye exclusion from the substrate pores was selected and a cross section of the substrate under the patch was imaged via a $\mathrm{z}$-linescan time series at $5 \mathrm{~s}$ intervals. Pyranine buffer containing the peptide in the specified concentration was added by pumping across the substrate. The first $750 \mu \mathrm{l}$ were discarded, after which the solution was circulated.

For the experiments with the voltage sensitive dye $\mathrm{DiOC}_{2}(3)$, undoped POPC GUVs were spread in PBS and $10 \mu \mathrm{M}$ dye was added manually. For imaging, membrane patches were observed with the $63 \mathrm{x}$ objective and time series were captured at 1 frame/s in the plane of the lipid bilayer. Fluorescence was excited at $488 \mathrm{~nm}$ and detected with the spectral detector in a range of 490-650 nm, split into 17 channels. During imaging, a defined potential between $-50 \mathrm{mV}$ and $+50 \mathrm{mV}$ was applied to the substrate.

For the experiments with pyranine trapped in the substrate under PSMs, undoped POPC GUVs were sedimented and spread in PBS containing $1 \mathrm{mM}$ pyranine. After $10 \mathrm{~min}$ of incubation, the chamber was gently rinsed with clear PBS until dye concentration was negligible. For imaging, membrane patches were observed with the $63 x$ objective and time series were captured at 1 frame/s in a plane several micrometers below the lipid bilayer. Fluorescence was excited sequentially at $405 \mathrm{~nm}$ and $458 \mathrm{~nm}$ and detected at 500-600 nm. A defined potential between $-75 \mathrm{mV}$ and $+75 \mathrm{mV}$ was applied to the substrate in several steps. Synchronization was achieved by connecting the external trigger output of the potentiostat to the input of the CLSM. 


\section{$4 \quad$ Results and Discussion}

\subsection{Muraymycin}

To gain insight into the role of the fatty acid sidechains in the activity of muraymycin, the fluorescently labeled sidechain (LSC) of muraymycin A1 ( $\omega$-hydroxyguanidino tridecanoic acid) has been previously synthesized and investigated in a model membrane system by Ries et al.. ${ }^{[72]}$ Adhered giant unilamellar vesicles were incubated with the labeled sidechain and characterized by confocal fluorescence microscopy. The study has suggested that the muraymycin A1-LSC accumulates at bilayers and has a measurable influence on them. The measure used by the authors for lipid bilayer destabilization or direct permeabilization by the LSC was the increase in the number of dye-filled GUVs after 40 min of incubation time. ${ }^{[72]}$ The goal of this thesis was to reproduce these results and then expand the experiment by introducing LSCs with differing functional end-groups and chain lengths. The LSCs were synthesized by the Ducho group of the Saarland University, following the established protocol. ${ }^{[72]}$
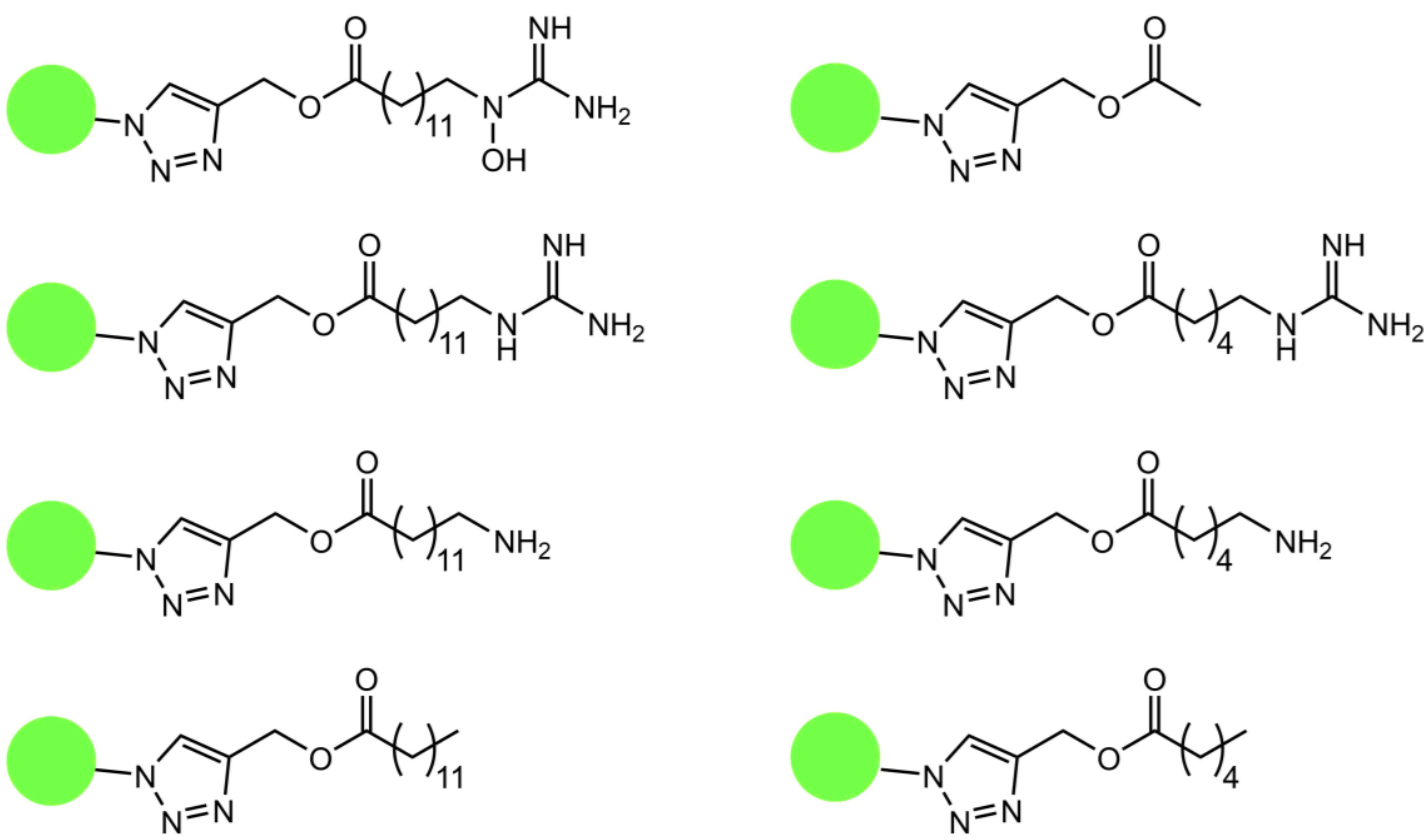

Figure 4.1: Overview of the AF488 labeled LSC derivates used in this work. Green circles represent the AF488 fluorophore coupled via a $\mathrm{C}_{6}$-linker (see Figure 3.4). 


\subsubsection{Influx of muraymycin lipid side chains into adhered GUVs}

The setup to measure bilayer permeabilization was comprised of vesicles, containing $0.1 \mathrm{~mol} \%$ biotinylated lipid, adhered on avidin coated silicon wafers. After addition of fluorescently labeled LSC to the buffer surrounding the vesicles, the influx of dye into the vesicles was observed via confocal microscopy. By using a relatively low magnification objective, a high number of adhered GUVs could be observed simultaneously over time. The observation plane was set several micrometers above the substrate, minimizing the influence of membrane fluorescence on the signal and allowing for a moderate z-drift over time (see Figure 4.2).

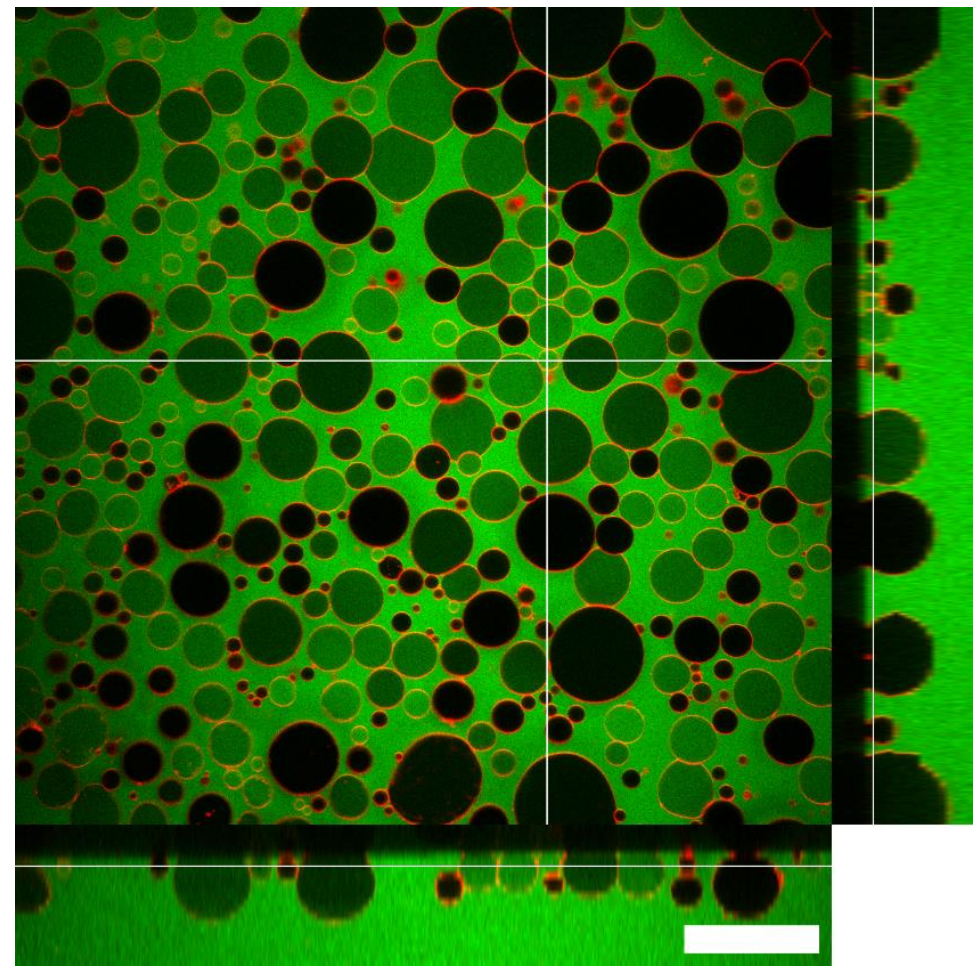

Figure 4.2 Orthogonal view of GUVs adhered on an avidin coated silicon wafer in PBS after addition of pyranine to the buffer. White lines indicate slice positions, scale bar $100 \mu \mathrm{m}$.

The mean fluorescence $F$ of the content of each GUV was normalized to $F_{0}$, the intensity of the surrounding solution. Vesicles were considered to be filled when the fill ratio, i.e. the normalized fluorescent intensity $F / F_{0}$, exceeded 0.5 . The observation time was set to 40 minutes. The experimental conditions, particularly for the adhesion of GUVs on the silicon substrate, were kept identical to the work of Ries et al..[72] 


\section{Results and Discussion}

First, blind experiments were conducted with both the water-soluble fluorescent dye pyranine and, as a direct analogue to the LSCs, the acetic acid conjugate AF488-acetate. Avidin coated silicon wafers were submerged in PBS and incubated with GUVs composed of POPC, doped with $0.1 \mathrm{~mol} \%$ biotin-PEG(2000)-DSPE and $0.1 \mathrm{~mol} \%$ TexasRed-DHPE. After addition of dye to the buffer, confocal micrographs of the vesicles were recorded in intervals of $120 \mathrm{~s}$ or less for $40 \mathrm{~min}$ (see chapter 3.3).
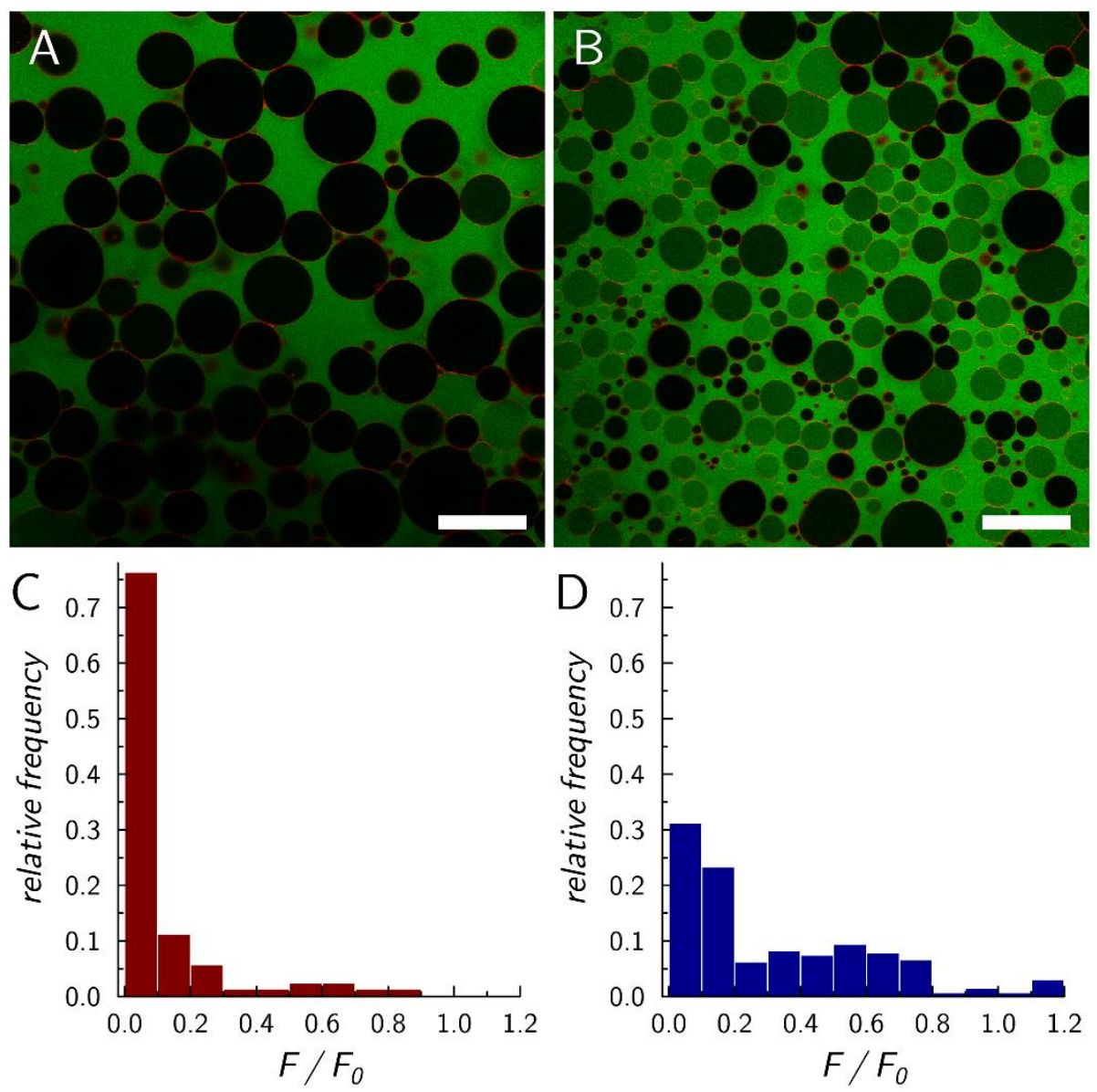

Figure 4.3 A and B: Confocal micrographs of GUVs adhered on avidin coated silica wafers. A: after $40 \mathrm{~min}$ incubation with $0.8 \mathrm{mM}$ AF488-acetate. B: after 5 min incubation with $0.8 \mathrm{mM}$ pyranine. Scale bars $100 \mu \mathrm{m}$. C and D: Histograms of the frequency distribution of the fill ratios $\mathrm{F} / \mathrm{F}_{0}$ of individual GUVs (bin size 0.1) C: after 40 min incubation with $0.8 \mathrm{mM}$ AF488-acetate. D: after 5 min incubation with $0.8 \mathrm{mM}$ pyranine.

One preparation showed a fraction of filled GUVs $\left(F / F_{0}>0.5\right)$ of $6.5 \%$ (see Figure $4.3 \mathrm{~A}$ and C) after $40 \mathrm{~min}$, very similar to the results of Ries et al., who reported a fraction of $6 \% .{ }^{[72]}$ The results of further control experiments were highly variable, however, and several preparations showed significant dye influx into a large fraction of adhered vesicles even 
shortly after dye addition (see Figure 4.3 B and D). As no membrane-disrupting effects are expected from both dyes, the elevated number of filled GUVs in some of the experiments can most likely be traced to mechanical defects in the bilayer at the adhesion interface. Incomplete coverage with avidin can lead to strong interactions between lipid head groups and the exposed silicon dioxide. These forces are capable of disrupting bilayer integrity, as evidenced by the spreading of vesicles on bare oxide surfaces. ${ }^{[94]}$

Development of a stable model system

Several methods were tested to increase the consistency between measurements. As a first step, avidin was exchanged for neutravidin, a deglycosylated derivate of the native protein. Despite its lower isoelectric point in comparison to avidin, it is readily immobilized on silica surfaces and can form coatings exceeding monolayer thickness. ${ }^{[95]}$ Additionally, to suppress unspecific interactions between the adhered bilayer and the substrate at defects in the protein layer, the neutravidin-coated silicon wafers were incubated with bovine serum albumin (BSA) before GUV adhesion. This approach was successful in immobilizing GUVs on the surface, but again showed highly inconsistent vesicle integrity between measurements (see Figure 4.4). 

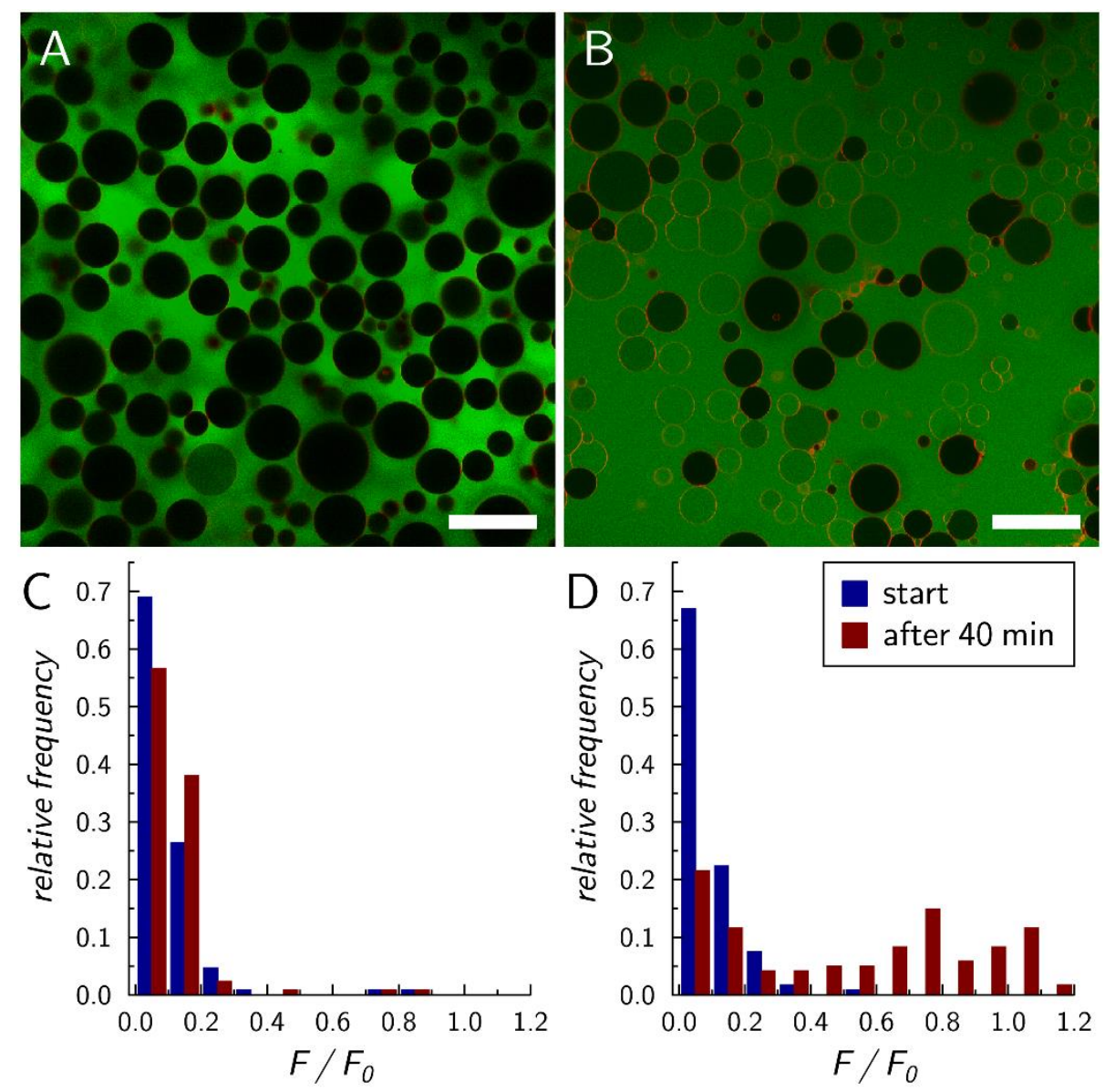

Figure 4.4: A and B: Confocal micrographs of adhered GUVs after 40 min of incubation with $0.8 \mathrm{mM}$ AF488-acetate. Both substrates were prepared identically with subsequent incubation in $0.1 \mathrm{mg} / \mathrm{ml}$ neutravidin $(30 \mathrm{~min}$ ) and $0.1 \mathrm{mg} / \mathrm{ml}$ BSA (30 min). Scale bars $100 \mu \mathrm{m} . \mathrm{C}$ and D: Relative frequencies of the fill ratios $\mathrm{F} / \mathrm{F}_{0}$ of individual GUVs at the start and after $40 \mathrm{~min}$, bin size 0.1. The fractions of filled GUVs $\left(\mathrm{F} / \mathrm{F}_{0}>0.5\right)$ after $40 \mathrm{~min}$ are $2.3 \%(\mathrm{n}=129)$ and $56 \%(\mathrm{n}=121)$, respectively.

The fraction of filled GUVs after 40 min was determined for three separate measurements to be $2.3 \%(\mathrm{n}=129), 35 \%(\mathrm{n}=80)$, and $56 \%(\mathrm{n}=121)$. Prolonged incubation with $0.1 \mathrm{mg} / \mathrm{ml}$ BSA overnight did also not result in good passivation (22\% filled GUVs after $20 \mathrm{~min}, \mathrm{n}=191$ ).

Instead of the direct attachment of avidin on the substrate, a more nuanced approach was evaluated. As avidin contains four binding sites for biotin, it can be attached to biotin-coated surfaces and still bind to biotinylated lipids. The coating of surfaces with biotin can be achieved with a wide variety of methods and in conjunction with other surface modifications, e.g. PEGylation. An established method is the coating of a glass substrate with a PEG layer containing 10 mol\% biotinylated PEG (see chapter 3.3.1).[74,96] This method enables the controlled attachment of avidin, while the PEG spacer suppresses any nonspecific interaction 
between the lipid bilayer and glass surface. Again, the deglycosilated variant neutravidin was used instead of the native protein. On PEGylated glass, GUV adhesion was more consistent and blind measurements yielded a relatively constant permeabilization rate with approx. $10 \%$ filled GUVs after $40 \mathrm{~min}$ (see Table 1). Most vesicles showed a fill ratio below 0.2 (see Figure 4.5).
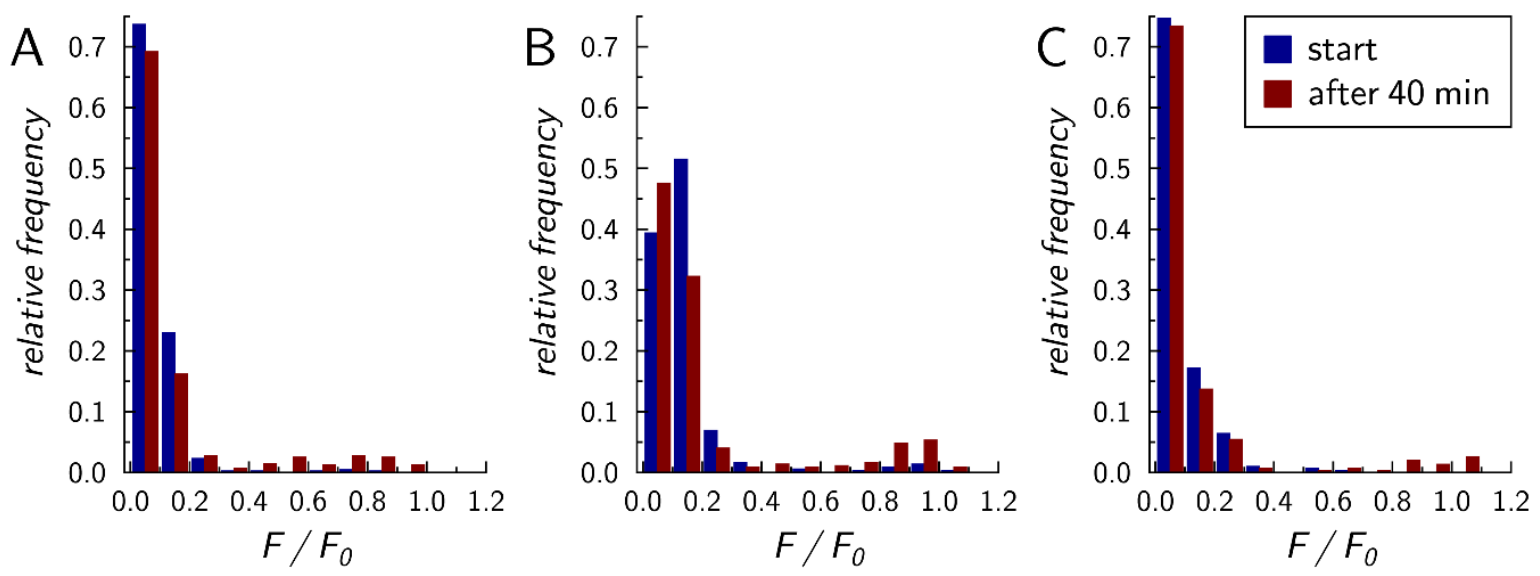

Figure 4.5: Distribution of fill ratios of adhered GUVs after 40 min of incubation with AF488-acetate for three separate preparations. GUVs (POPC with $0.1 \mathrm{~mol} \%$ biotin-PEG(2000)-DSPE) adhered on PEGylated glass, incubated with neutravidin.

Measurement of LSC translocation into GUVs

With a stable model system in hand, the translocation of the different lipid side chains into adhered GUVs was investigated. After vesicle adhesion, the labeled LSCs were added to the surrounding buffer and the vesicles were observed for $40 \mathrm{~min}$. The experiment was performed with the $\omega$-hydroxyguanidino-, guanidino-, and amino-LSC conjugates and with the AF488acetate as negative control. The adhered GUVs were composed of POPC or POPC/POPE (70/30 mol\%) and doped with $0.1 \mathrm{~mol} \%$ biotinylated DSPE. Each LSC was measured three times in POPC and only twice in POPC/POPE 70/30, due to the low amount of available LSC-conjugates. Two measurements were excluded from the dataset as the vesicles showed lateral movement, which indicates low adhesion strength. The results are summarized in Table 1. 


\section{Results and Discussion}

Table 1: Summarized results of the GUV permeabilization experiments on PEG-coated glass. A1: $\omega-$ hydroxyguanidino-LSC, A3: $\omega$-guanidino-LSC

\begin{tabular}{|c|c|c|c|c|c|c|c|c|}
\hline \multirow[t]{2}{*}{$\begin{array}{l}\text { Lipid } \\
\text { composition }\end{array}$} & \multirow[t]{2}{*}{$\begin{array}{l}\text { LSC- } \\
\text { conjugate }\end{array}$} & \multicolumn{2}{|c|}{ 1. preparation } & \multicolumn{2}{|c|}{ 2. preparation } & \multicolumn{2}{|c|}{ 3. preparation } & \multirow[t]{2}{*}{$\begin{array}{l}\text { Mean fraction } \\
\text { of } \mathrm{F} / \mathrm{F}_{0}>0.5\end{array}$} \\
\hline & & $\mathrm{F} / \mathrm{F}_{0}>0.5$ & $\mathrm{n}$ & $\mathrm{F} / \mathrm{F}_{0}>0.5$ & $\mathrm{n}$ & $\mathrm{F} / \mathrm{F}_{0}>0.5$ & $\mathrm{n}$ & \\
\hline \multirow[t]{4}{*}{$\overline{\mathrm{POPC}}$} & Acetate & $10.0 \%$ & 489 & $7.0 \%$ & 315 & $14.3 \%$ & 379 & $10.4 \%$ \\
\hline & A1 & $5.3 \%$ & 342 & $16.6 \%$ & 410 & $10.3 \%$ & 319 & $10.7 \%$ \\
\hline & A3 & $11.4 \%$ & 246 & $2.6 \%$ & 228 & $15.2 \%$ & 230 & $9.7 \%$ \\
\hline & Amino & $8.1 \%$ & 198 & $11.4 \%$ & 236 & $4.7 \%$ & 465 & $8.1 \%$ \\
\hline \multirow[t]{4}{*}{$\mathrm{PC} / \mathrm{PE}$} & Acetate & - & - & $1.6 \%$ & 304 & $4.9 \%$ & 287 & $3.3 \%$ \\
\hline & A1 & - & - & $5.3 \%$ & 434 & $9.5 \%$ & 315 & $7.4 \%$ \\
\hline & A3 & - & - & - & - & $1.5 \%$ & 399 & $1.5 \%$ \\
\hline & Amino & - & - & - & - & $2.0 \%$ & 545 & $2.0 \%$ \\
\hline
\end{tabular}

On the whole, no significant influence of the different LSC-conjugates on the ratio of filled POPC GUVs was found, as the variations between preparations mostly exceeded those between the LSCs. Vesicles with 30 \% POPE were generally more stable than POPC GUVs and here a slightly elevated fill ratio was measured for the $\omega$-hydroxyguanidino-LSC. Due to the limited number of experiments this finding is not conclusive.

In the control experiments, the dye content of GUVs increased rapidly and discontinuously in most cases (see Figure 4.6 A), which suggests that small mechanical defects at the adhesion interface are the main mechanism of dye transport. The $\omega$-hydroxyguanidino-LSC on the other hand showed significantly slower dye influx in the majority of filled GUVs (see Figure 4.6 C). However, as the ratio of filled GUVs was not elevated, this finding can most likely be attributed to the partitioning of dye into the inner leaflet of the bilayer upon entering the vesicle. A continuous slow increase in the fill ratio across all GUVs, indicating a direct translocation of labeled LSCs across the intact bilayer, was not observed on the timescale of the experiment. 
It can be concluded that no significant membrane destabilization was observed for any of the LSC-conjugates in the low micro-molar concentration range that was used in this study.
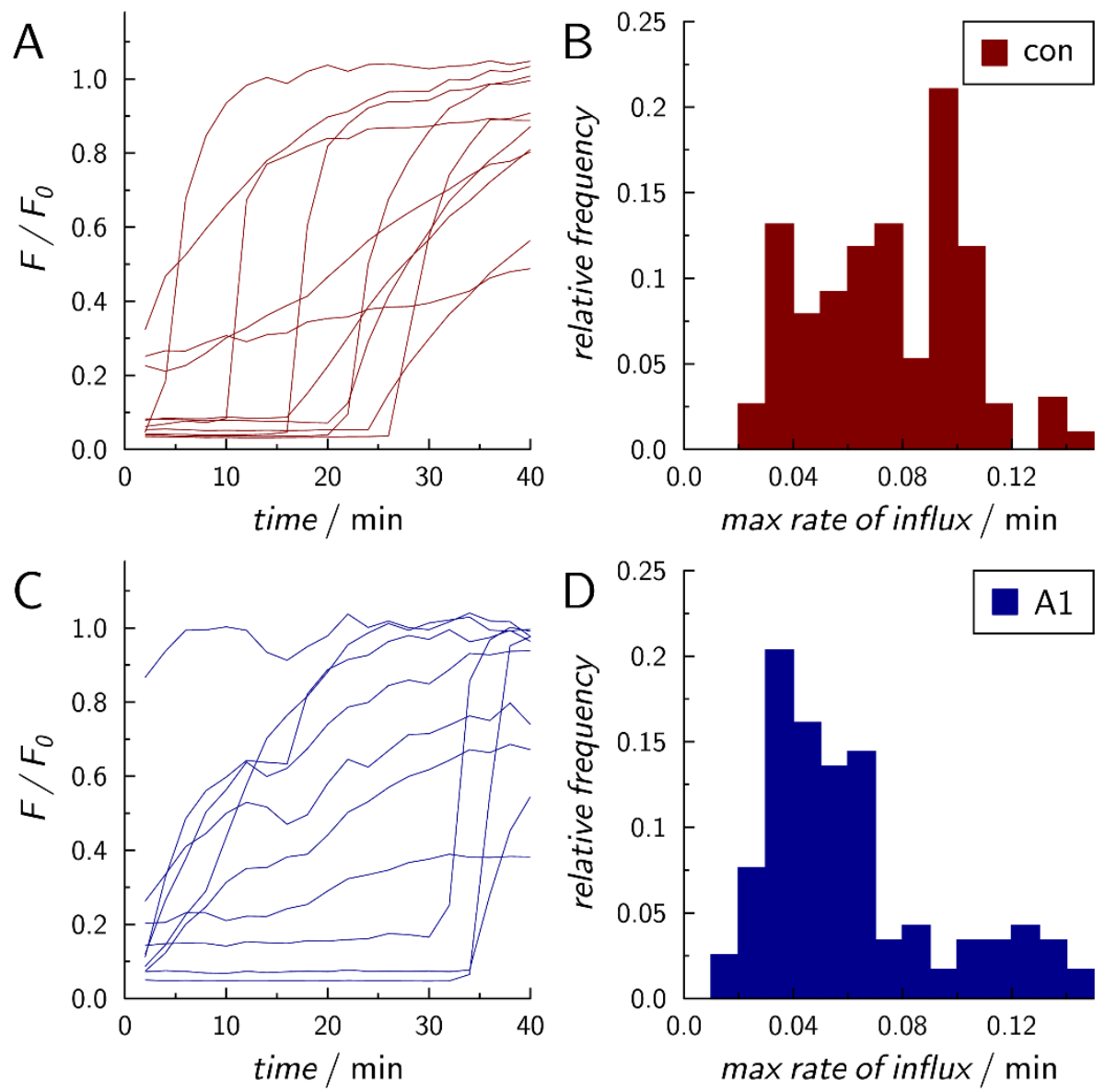

Figure 4.6: A and C: Exemplary traces of the normalized fill ratio of individual GUVs over time for AF488-acetate and the w-hydroxyguanidino-LSC conjugate A1. B and D: Distribution of the maximum rate of dye influx $\mathrm{dF} / \mathrm{dt}$ into individual GUVs. Included are GUVs from the combined experiments with $\mathrm{F} / \mathrm{F}_{0}<0.75$ at the start and $\mathrm{F} / \mathrm{F}_{0}>0.5$ after $40 \mathrm{~min}$. The median influx rate of $\omega-$ hydroxyguanidino-LSC $(\mathrm{A} 1)$ is significantly smaller than that of the control $(\mathrm{P}<0.001$, Wilcoxon rank-sum test, $\mathrm{n}=76$ (control), $\mathrm{n}=118(\mathrm{~A} 1)$ ). 


\section{Results and Discussion}

\subsubsection{Partitioning of labeled muraymycin lipid side chains into lipid bilayers}

The accumulation of dye at the lipid bilayer was observed to be dependent on the specific LSC and the vesicle lipid composition. This accumulation is relatively weak and the fluorescent signal from the membrane is on the same order of magnitude as the background signal from the solution. Therefore, confocal microscopy is an ideal method to measure the accumulation, as even weak signals can be detected in fluorescent solution as long as they are above the background noise.

The interaction of the LSCs with the bilayer can be interpreted either as an adsorption on a surface or as a partitioning between the aqueous phase and the lipid bilayer. Due to the fast and reversible accumulation that showed no saturation in the concentration range used, the interaction was regarded as a partitioning between two phases. In equilibrium, this partitioning can be quantified with a coefficient $P$ that is the quotient of the concentrations of fluorescent conjugate in the lipid bilayer and in the aqueous solution (equation ( 3 )).

$$
P=\frac{c_{\text {membrane }}}{c_{\text {aqueous }}}
$$

When observing a thin membrane, the observation volume of the microscope, given by its point-spread function (PSF), is only partly filled by the membrane. Therefore, the specific volumetric fluorescence intensity of the membrane is systematically underestimated, which precludes a direct comparison of the measured membrane fluorescence with the intensity of the bulk solution. Instead, the quotient of the membrane fluorescent intensity $I_{\text {membrane }}$ and the solution intensity $I_{\text {aqueous }}$ gives an apparent partition coefficient $P_{a p p}$ which is directly proportional to the actual $P$ (equation ( 4 )).

$$
P_{\text {app }}=\frac{I_{\text {membrane }}}{I_{\text {aqueous }}}
$$

As $c_{\text {membrane }}$ is an area concentration, $P$ has the dimension of meters, but $P_{a p p}$ was treated as dimensionless. In the case of adhered GUVs, $I_{\text {aqueous }}$ is not necessarily equal on both sides of the bilayer. As the equilibrium between membrane and solution is reached much faster than 
any potential flip-flop for the LSC conjugates, each leaflet is assumed to show independent partitioning with the adjacent solution. The two leaflets of the membrane are indistinguishable with optical means and only their combined signal is accessible. Under the assumption of a constant $P_{a p p}$ and leaflets of equal size and composition, two approaches are possible to define $P_{a p p}$. The leaflets can be treated as two separate phases, each in an equilibrium with their respective aqueous phase.

$$
I_{\text {membrane }}=P_{\text {app }} * I_{\text {aqueous } 1}+P_{\text {app }} * I_{\text {aqueous } 2}
$$

Alternatively, the leaflets can be treated as a single lipid phase that is in equilibrium with a single aqueous phase. The concentration of this single aqueous phase is then the average of the two actual solution concentrations.

$$
I_{\text {membrane }}=P_{\text {app }} * \frac{I_{\text {aqueous } 1}+I_{\text {aqueous } 2}}{2}
$$

The second approach given by equation ( 6 ) differs in a factor of two for $P_{a p p}$, which is of no consequence to the interpretation however, as the actual proportionality factor to $\mathrm{P}$ is not known. This approach was chosen for the further analysis as it provides a more intuitive definition for $P_{a p p}$, especially in the edge cases where the GUV interior shows no fluorescence at all or the same as the background.

The fact that the membrane is thinner than the PSF has another consequence for the interpretation of data. The observed signal is the sum of the bilayer signal and that of the solution on both sides of it. To extract the actual membrane intensity, the local background has to be subtracted. As the volume of the membrane is negligible in comparison to the observation volume, the background intensity at the membrane can be directly approximated as the mean of the solution intensities adjacent to the membrane.

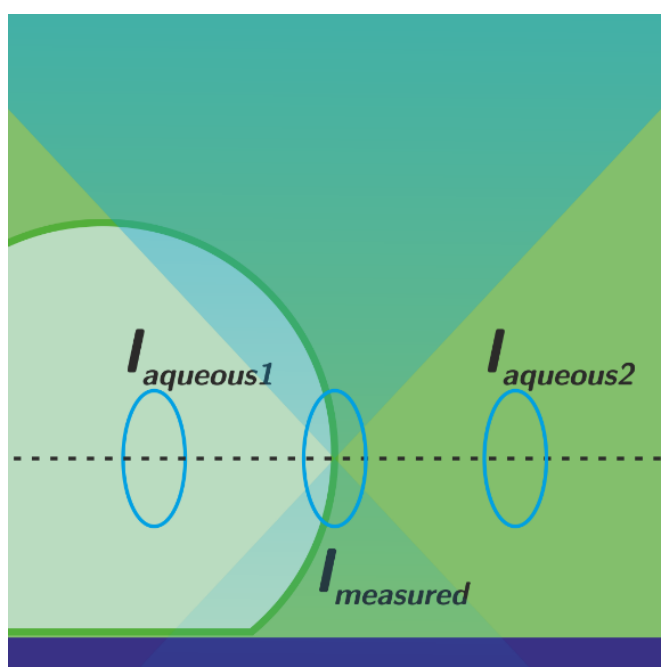

Figure 4.7: Schematic of an adhered GUV and its relation to the PSF of a CLSM. 
4 Results and Discussion

$$
I_{\text {membrane }}=I_{\text {measured }}-\frac{I_{\text {aqueous } 1}+I_{\text {aqueous } 2}}{2}
$$

Under these assumptions, the apparent partition coefficient can be calculated from the measured membrane intensity $I_{\text {measured }}$ and the mean of the solution intensities outside and inside the vesicle $I_{\text {background }}$ as follows:

$$
P_{\text {app }}=\frac{I_{\text {measured }}-I_{\text {background }}}{I_{\text {background }}}
$$

Two preliminary tests were performed to ensure a correct interpretation of the measured data. The assumption of a direct proportionality between solution and membrane concentration was investigated by varying the LSC concentration. The partition coefficient of guanidino-LSC in POPC bilayers was analyzed for different concentrations of the LSC ranging from 0.4 to $3.2 \mu \mathrm{M}$.

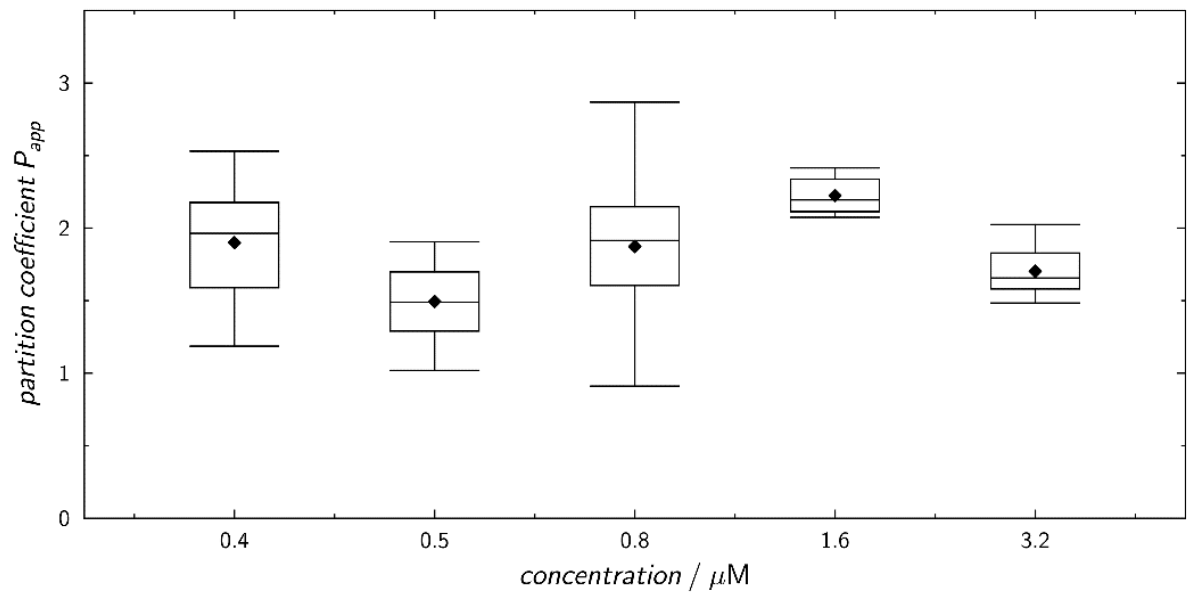

Figure 4.8: Apparent partition coefficient for the guanidino-LSC in POPC vesicles at concentrations of $0.4,0.5,0.8,1.6$ and $3.2 \mu \mathrm{M}, \mathrm{n}=25,8,39,9$, and 11 respectively.

No threshold or saturation was observed, and further experiments were conducted under the assumption of a linear relationship between dye concentration in solution and in the bilayer. This is analog to the partitioning of the dye between two phases and the normalized membrane intensity can indeed be interpreted as an apparent partition coefficient $P_{a p p}$. The LSC concentration was kept constant at $0.8 \mu \mathrm{M}$ for all other experiments. Some variation in absolute fluorescence of the solutions was observed between measurements, at least partly 
due to incomplete mixing at the substrate surface. These variations were all in the range tested for linearity and considered to be of no consequence to the partitioning equilibrium.

The second assumption was that each bilayer leaflet is independently in a fast equilibrium with the adjacent aqueous phase and the measured GUV membrane intensity is the sum of both leaflet intensities. The apparent partition coefficient can then be calculated from the mean of the solution intensities on both sides of the bilayer. The assumption was tested with the $\omega$-hydroxyguanidino-LSC data by analyzing the relationship between the membrane intensity, normalized to the outer solution intensity, and the fill ratio of the GUV (Figure 4.9). In equilibrium, the expectation is a distribution with a certain positive membrane intensity at a fill ratio of zero and a doubled membrane intensity at a fill ratio of 1 . In other words, the y-axis intercept and the slope of the distribution should be equal.

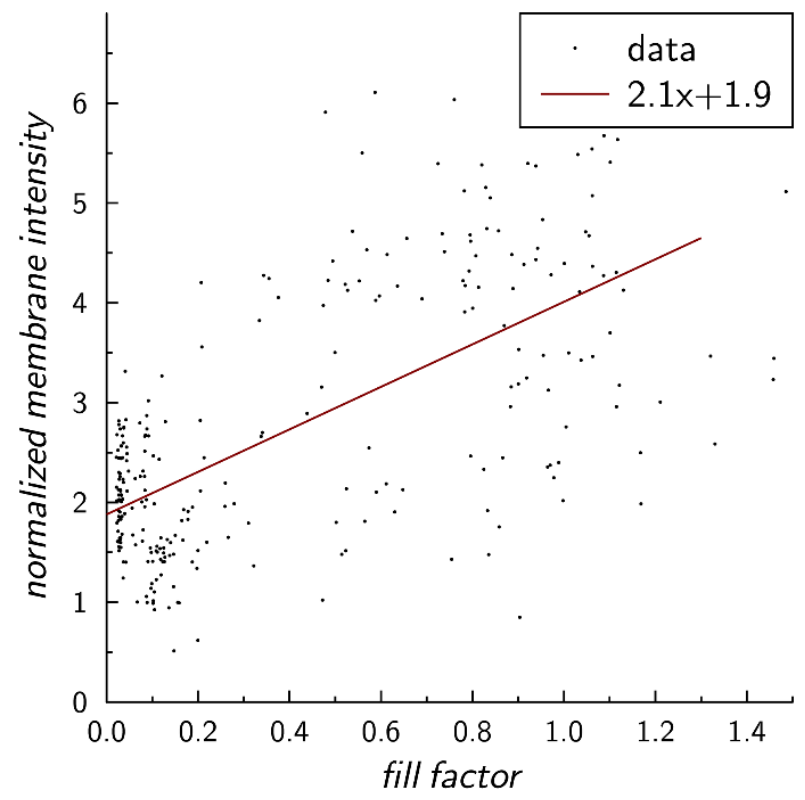

Figure 4.9: Membrane intensity divided by the solution intensity plotted against the fill factor for A1LSC and POPC. The line is derived from a linear least-squares fit of the dataset to $\mathrm{y}=\mathrm{mx}+\mathrm{c}(\mathrm{m}=2.1$, $\mathrm{c}=1.9)$.

The regression line of the data shows a slope of 2.1 and an intercept of 1.9 , confirming the assumed proportionality and that an equilibrium is present between each leaflet and the adjacent solution, but not or much slower between leaflets.

In subsequent experiments, the partition coefficients of the $\omega$-hydroxyguanidino-, guanidino-, and amino-tridecanoic acid LSCs were determined for adhered vesicles composed of POPC, 


\section{Results and Discussion}

POPC/POPE (70/30), and POPC/POPE (40/60). For the unfunctionalized tridecanoic acid conjugate, $P_{a p p}$ was measured in POPC and POPC/POPE (70/30) vesicles. The hexanoic acid derivatives were investigated with POPC vesicles only. The adhesion procedure and microscope setup (see chapter 3.3.2) were kept constant and each combination was measured with at least two preparations. The substrates were systematically screened and vesicles were imaged individually in their equatorial plane. When possible, partly or completely filled vesicles were imaged preferentially, due to the low signal to noise ratio for empty vesicles. The fluorescence micrographs were evaluated with a custom MATLAB script (see chapter 3.3.3) which calculated the apparent partition coefficient $P_{a p p}$ for each GUV from vertical line scans which were fitted with the function described in equation ( 2 ).

Influence of $\omega$-functionalization on lipid side chain accumulation

The $\omega$-hydroxyguanidino-LSC showed the highest affinity of the $\omega$-functionalized tridecanoic acid conjugates to lipid bilayers composed of POPC. Guanidine functionalized sidechains showed significantly lower partitioning, with amino functionalized LSCs following this trend The partition coefficients are shown as boxplots in Figure 4.10.

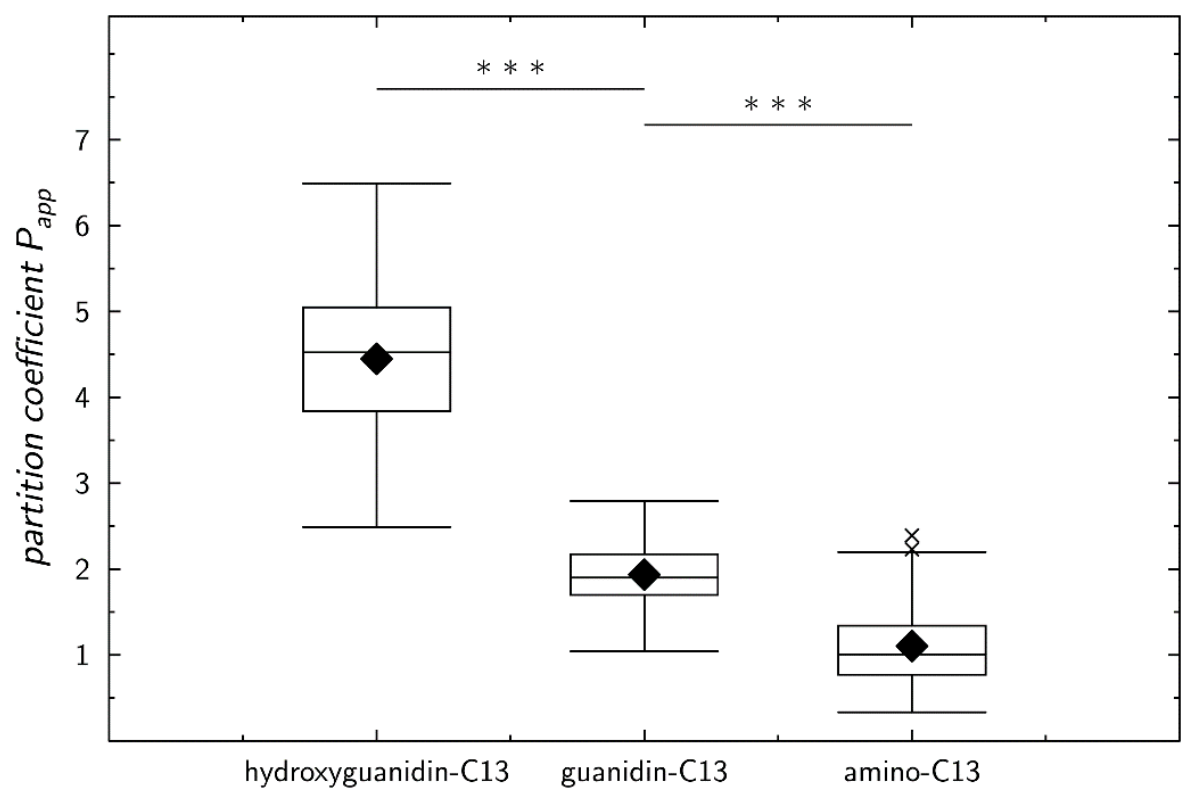

Figure 4.10: Boxplots of the apparent partition coefficients $P_{a p p}$ of different $\omega$-functionalized AF488LSC conjugates in GUVs composed of POPC. Each box shows upper and lower quartiles, median (line) and mean (diamond). Whiskers show the data range, except for outliers more than 1.5 times the interquartile range from the box (crosses). The medians of $P_{a p p}$ are $4.5(\mathrm{n}=54)$ for $\omega-$ hydroxyguanidino-, $1.9(\mathrm{n}=118)$ for $\omega$-guanidino- ,and $1.0(\mathrm{n}=98)$ for $\omega$-amino-LSC conjugate. All pairs are significantly different $(\mathrm{P}<0.001$, Wilcoxon rank-sum test). 
The unfunctionalized tridecanoic acid AF488 conjugate is visibly fluorescent in organic solvents, but the bright green color changes instantly to a non-fluorescent red upon addition of water. The probable cause for this change is self-quenching due to micelle formation. Nevertheless, measurements of an apparent partition coefficient were possible by performing subsequent confocal scans with different laser intensities. Apparent partition coefficients in excess of 100 were measured, showing strong interaction with the bilayer.

A quantitative comparison between absolute membrane intensities of tridecanoic acid- and $\omega$-functionalized conjugates is difficult, as both laser intensity and detector gain varied between measurements and detector gain is a nonlinear factor. A calibration with an external standard was not necessary for the functionalized conjugates and was, therefore, not performed. Nevertheless, a general trend is found in the data after addition of $0.8 \mu \mathrm{M}$ AF488conjugate: membrane-bound tridecanoic acid consistently showed a higher fluorescence signal than the $\omega$-functionalized conjugates, even at lower gain and lower laser power.

For completely soluble conjugates the apparent partition coefficient is defined under the assumption of a direct proportionality between the concentrations and fluorescent intensities:

$$
P=\frac{c_{\text {membrane }}}{c_{\text {aqueous }}} \sim \frac{I_{\text {membrane }}}{I_{\text {background }}}=P_{\text {app }}
$$

For a micellar solution, however, the partitioning is governed by the concentration of free monomers, which is limited by the critical micelle concentration CMC. For any concentration above the CMC, $I_{b a c k g r o u n d}$ is the sum of the normal fluorescence of the monomers $I_{C M C}$ and the, probably non-zero, intensity of the quenched micelles:

$$
\begin{gathered}
I_{\text {background }}=I_{\text {micelles }}+I_{C M C} \\
I_{\text {micelles }} \geq 0
\end{gathered}
$$

The measured background intensity is therefore always greater than or equal to the intensity of free monomer $I_{C M C}$ :

$$
I_{\text {background }} \geq I_{C M C} \sim c_{\text {monomer }}
$$




\section{Results and Discussion}

As the proportionality between $\mathrm{c}_{\text {membrane }}$ and $I_{\text {membrane }}$ should remain unchanged, the partition coefficient $I_{\text {membrane }} / I_{\text {background }}$ is the lower bound for the actual $P_{a p p}$, as defined for the soluble conjugates.

$$
P_{\text {app }}=\frac{I_{\text {membrane }}}{I_{C M C}} \geq \frac{I_{\text {membrane }}}{I_{\text {background }}}
$$

To test these assumptions, a preliminary experiment was conducted and measurements were performed with different concentrations of unfunctionalized tridecanoic acid AF488 (0.2 $\mu \mathrm{M}$, $0.4 \mu \mathrm{M}$ and $0.8 \mu \mathrm{M})$. The membrane intensities were partially saturated, but the background intensities could be compared. A plot of $I_{\text {background }}$ against the concentration shows a positive slope and a positive y-axis intercept (see Figure 4.11), which supports the notion of a nonzero $I_{\text {micelles }}$ and a $\mathrm{CMC}<0.8 \mu \mathrm{M}$.

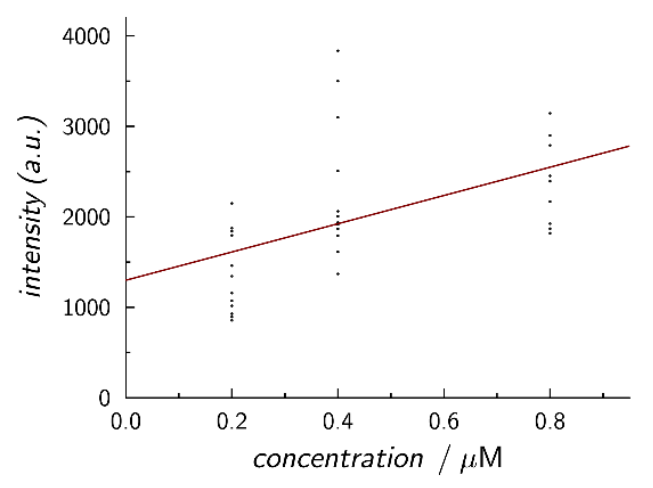

Figure 4.11: Linear regression of the fluorescent intensity of solutions of tridecanoic acid AF488conjugate in PBS at concentrations of $0.2 \mu \mathrm{M}, 0.42 \mu \mathrm{M}$ and $0.8 \mu \mathrm{M}$.

Subsequent measurements were done at $0.8 \mu \mathrm{M}$. The measurements showed a relatively high variation of $P_{a p p}$ with data in the range from 56 to 279 and an interquartile range from 130 to 196 (see Figure 4.17).

To explain this high variation, it must be considered that the fluorescence intensity of the partially quenched micelles complicates the measurements. In general, the local dye concentration around the vesicles is not always at exactly $0.8 \mu \mathrm{M}$ due to uncomplete mixing and the dye concentration is also lower inside only partially filled vesicles. For the soluble 
conjugates, these deviations have no effect on $P_{a p p}$, as any variation in the aqueous solution is in a fast equilibrium with the membrane. For the micellar solution, however, a variation in micelle concentration does not directly influence the equilibrium between monomers in solution and in the membrane. Consequently, for non-zero micelle fluorescence, a variation in concentration results in a variation in $P_{a p p}$.

Errors stemming from the additional fluorescence signal of micelles that are not in a direct equilibrium with the lipid bilayer should only affect the magnitude of the systematic underestimation of $P_{a p p}$. Therefore, the measured partition coefficient $\mathrm{I}_{\text {membrane }} / \mathrm{I}_{\text {background }}$ is the lower bound for the actual $P_{a p p}$ of the tridecanoic derivate. Both concentration and partition coefficient are at least one order of magnitude higher than for the functionalized conjugates.

Influence of lipid side chain length on their accumulation In addition to the tridecanoic acid derivatives, a set of shorter lipid side chain conjugates with and without $\omega$-functionalization was characterized. The $\omega$-guanidino- and $\omega$-aminohexanoic acid conjugates showed no detectable accumulation in POPC bilayers (see Figure 4.12). At the signal to noise ratio of the obtained micrographs, this suggests a $P_{a p p}$ smaller than 0.1 for both conjugates. 

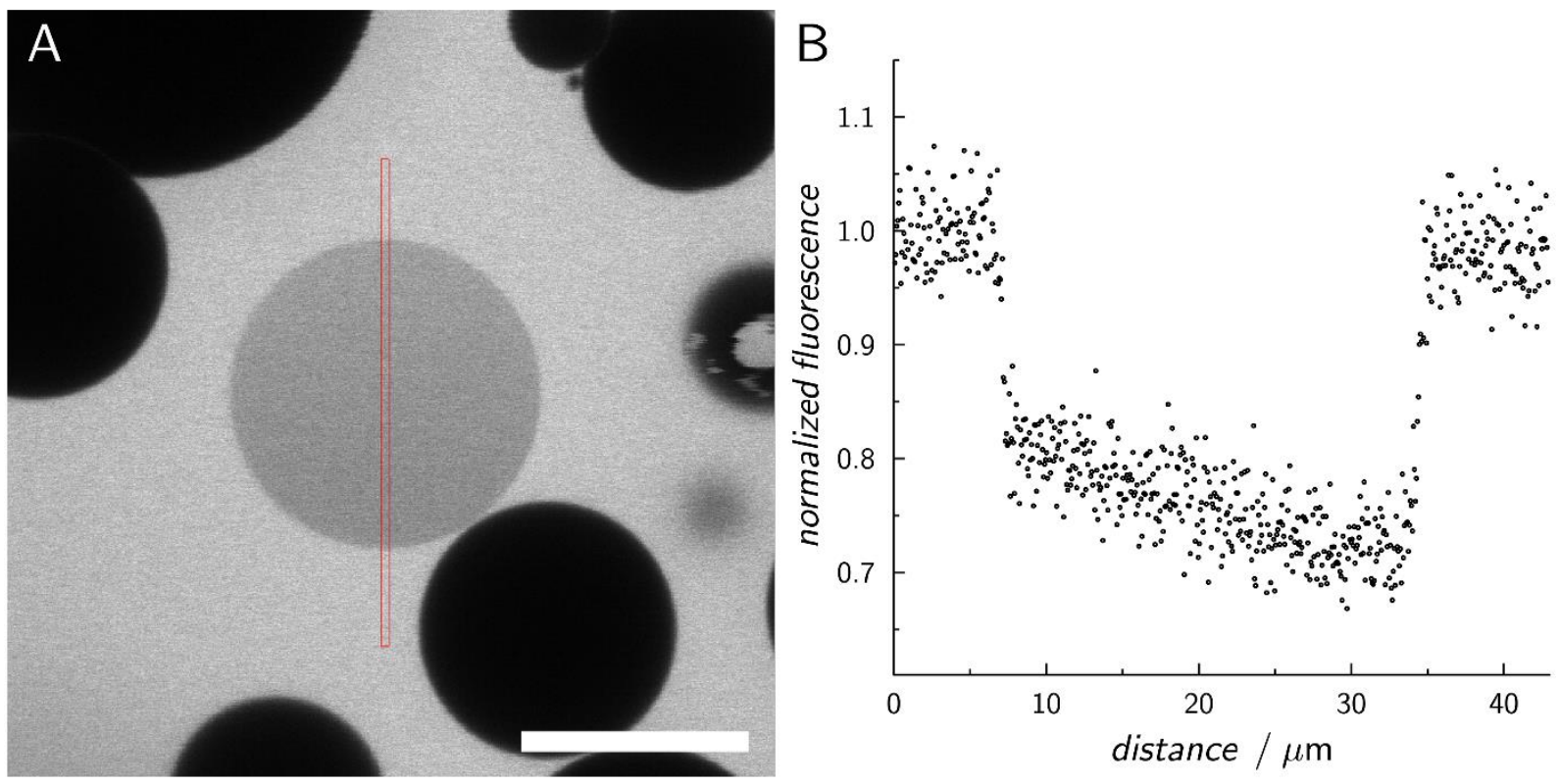

Figure 4.12 A: Confocal micrograph of a POPC-GUV incubated with $\omega$-guanidino hexanoic acid AF488 conjugate. Scale bar $20 \mu \mathrm{m}$. B: Intensity profile of the ROI shown in A.

The unfunctionalized hexanoic acid conjugate showed slight partitioning, which could only be detected in GUVs with high fill ratios (see Figure 4.13A). Due to the low signal to noise ratio, the coefficient cannot be reliably quantified (see Figure 4.13B). A rough estimation sets $P_{a p p}$ at the order of 0.1 , well below any of the $\mathrm{C}_{13}$-derivatives.
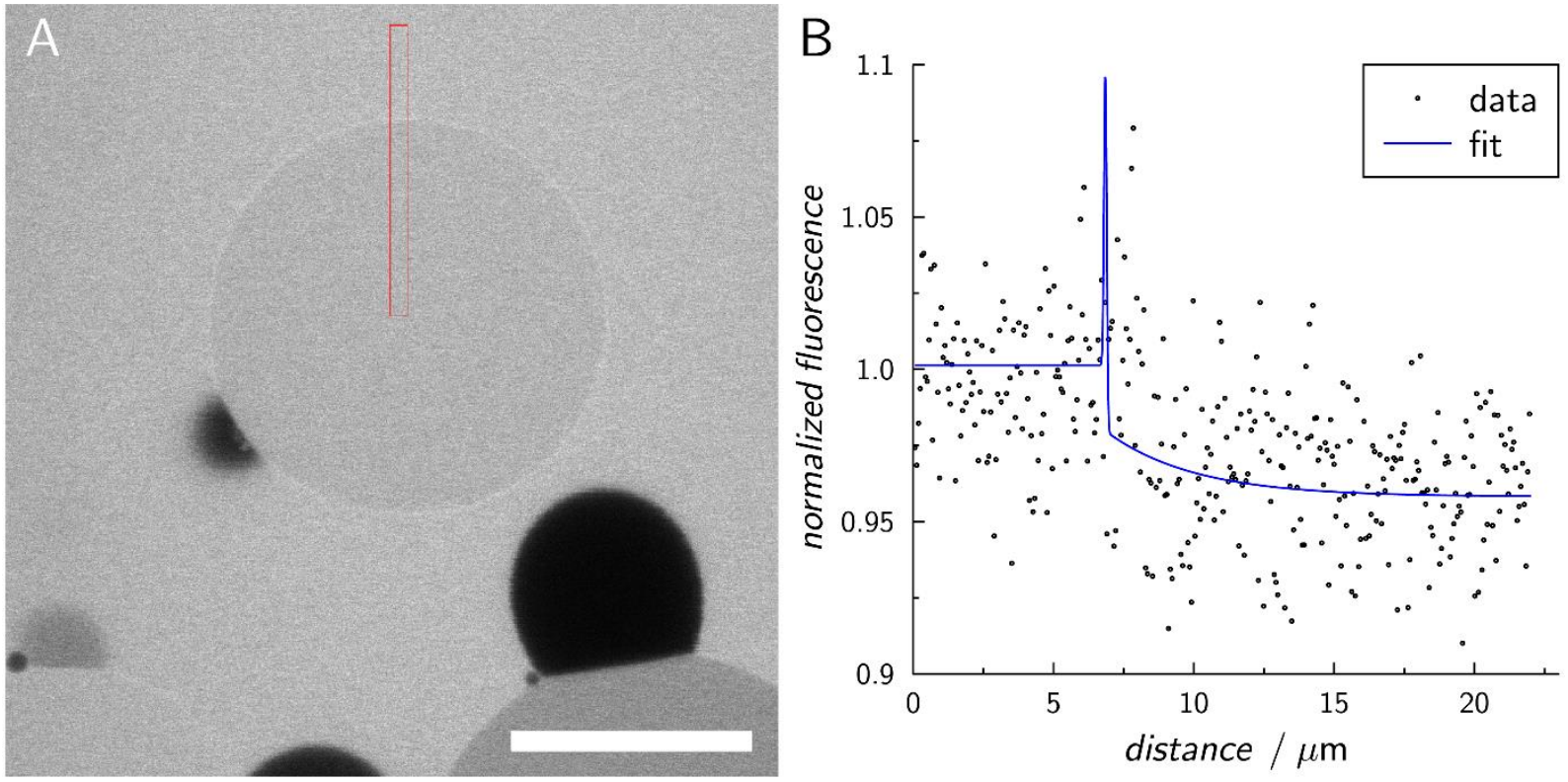

Figure 4.13: A: Confocal micrograph of a POPC-GUV incubated with hexanoic acid AF488 conjugate. Scale bar $20 \mu \mathrm{m}$. B: Intensity profile and data fit of the ROI shown in A. 
In an attempt to quantify the accumulation of the very weakly interacting $\mathrm{C}_{6}$-LSC derivatives, a modified measurement technique using Förster resonance energy transfer $(\mathrm{FRET})^{[97]}$ in conjunction with the CLSM was evaluated. The reasoning behind using this measurement technique was that, depending on its state, the LSC would interact to a different degree with a membrane bound dye. While the accumulated LSC can interact strongly with another membrane bound dye, the LSC in the surrounding aqueous solution can only interact with the dye in a layer a few nanometers thick, much thinner than the PSF of the microscope. Therefore, the FRET signal should greatly enhance the contrast

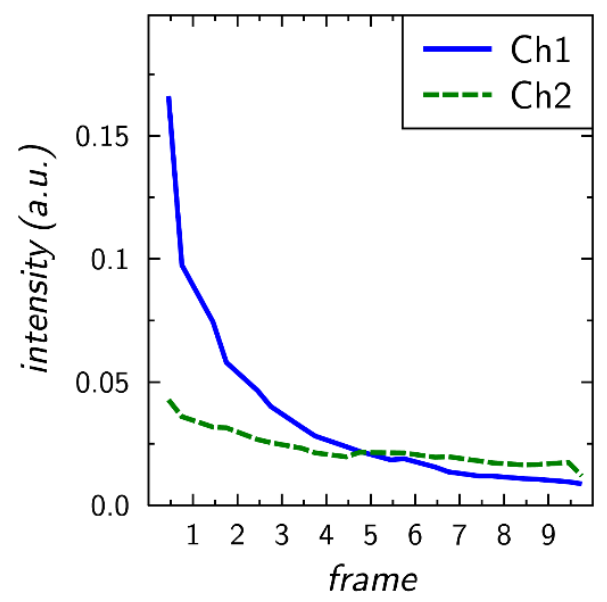

Figure 4.14: Fluorescence intensity of membrane bound Atto390 (0.01 mol\%) in consecutive frames of a time series of confocal micrographs. between membrane and background in comparison to the absolute LSC fluorescence measured up to this point. Two FRET pair variants were tested with the $\omega$-hydroxyguanidino tridecanoic acid conjugate and compared to the non-FRET system. First, Atto390DPPE was included in the vesicles as a donor dye in concentrations of 2 and $0.01 \mathrm{~mol} \%$. Adhered GUVs were imaged before and after incubation with LSC by exciting at $405 \mathrm{~nm}$ and recording both donor and acceptor fluorescence in two separate channels (420$470 \mathrm{~nm}, 500-550 \mathrm{~nm})$. In principle, due to energy transfer to the AF488-conjugate, a decrease in the blue channel fluorescence after LSC addition should be observed. Furthermore, the LSC fluorescence in the green channel should be more intensive at the membrane, with a direct proportionality to the Atto390 signal in addition to the partition coefficient. These expectations could not be quantified in the actual measurements, however, due to excessive bleaching of Atto390 and severe crosstalk between the channels. The Atto390 emission reached into the green channel and masked the LSC fluorescence completely at $2 \mathrm{~mol} \%$ and to some degree at $0.01 \mathrm{~mol} \%$. This crosstalk can be mitigated by subtraction of a constant fraction of the blue channel signal but is a source of noise and potential error. This becomes apparent when examining only the bleaching of Atto390, as it apparently was accompanied by a red shift in the emission, leading to nearly constant absolute crosstalk into the green channel with progressing bleaching (see Figure 


\section{Results and Discussion}

4.14). This observation implies that the relative crosstalk is not constant, which precludes the quantitative subtraction of Atto390 emission from the green channel. At the low Atto390 concentrations necessary to keep crosstalk low and still be able to detect the acceptor signal, bleaching cannot be avoided completely with the CLSM and the Atto390/AF488 FRET pair was thus deemed unsuitable for the analysis of LSC accumulation at lipid bilayers.

The second FRET pair that was evaluated consisted of the LSC as the donor and TexasRedDPPE as the membrane bound acceptor. Again, two-channel images were recorded, with excitation at $488 \mathrm{~nm}$ and detection at 500-540 $\mathrm{nm}$ for the green channel and 600-700 $\mathrm{nm}$ for the red channel. In this pair, the accumulation of LSC at the membrane should result in an increased fluorescence in the red channel due to energy transfer from excited AF488 to the rhodamine dye. Bleaching of the donor dye was of no concern, as, firstly, AF488 does not bleach as easily as Atto390 due to the longer wavelength excitation and secondly it is in a fast equilibrium with the surrounding solution. There was, however, considerable crosstalk between the two channels, both from AF488 emission bleeding into the red channel and from direct excitation of TexasRed with the $488 \mathrm{~nm}$ laser. At a TexasRed concentration of $0.5 \mathrm{~mol} \%$ in the GUVs, its excitation by $488 \mathrm{~nm}$ masked any FRET signal completely. At lower concentrations $(0.01 \mathrm{~mol} \%)$ the FRET could be detected as an increase of red membrane intensity after addition of LSC (see Figure 4.15). Even before adding the LSC, the initial membrane intensity in the red channel was above zero due to direct excitation of TexasRed at $488 \mathrm{~nm}$. After LSC addition, the main source of increased membrane fluorescence in the red channel was crosstalk from LSC emission, which was also present in the surrounding solution (see Figure 4.15, right side) and therefore did nothing to enhance the contrast between solution and bilayer. Subtracting the emission crosstalk calculated from the solution signal gave a rough estimate for the FRET efficiency. While the TexasRed membrane signal was nearly doubled by LSC addition (see Figure 4.15, middle), the increase due to FRET from the A1-LSC was only approximately $10 \%$ of the initial intensity in the red channel. 


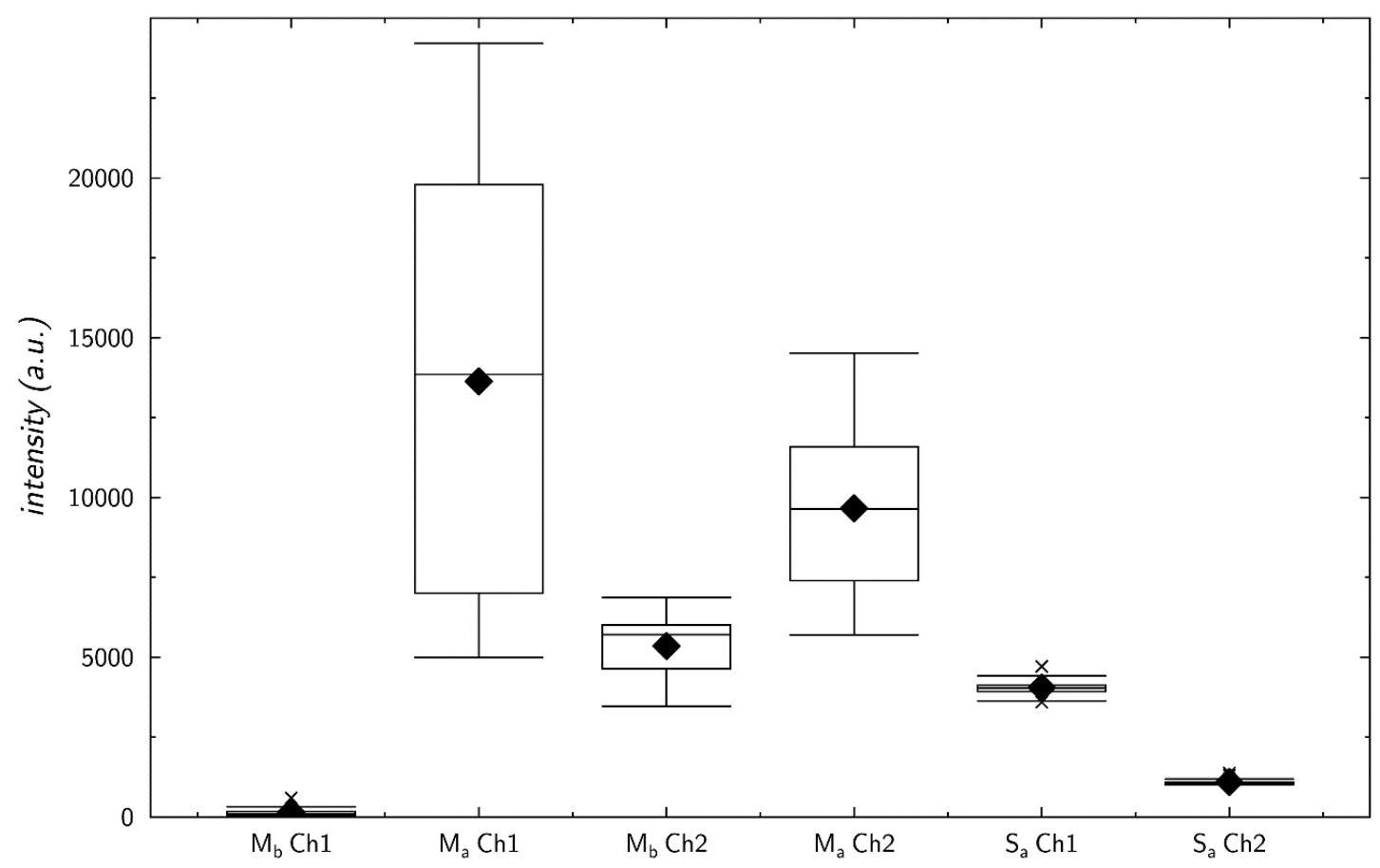

Figure 4.15 Fluorescence intensity of the GUV membrane (M, left side) and aqueous solution (S, right side) for a FRET-pair of 0.01 mol\% TexasRed (membrane-bound) and $0.8 \mu \mathrm{M} \omega$-hydroxyguanidinoLSC. Recorded on the green channel (Ch1) and red channel (Ch2) before $\left(\mathrm{M}_{\mathrm{b}}\right)$ and after $\left(\mathrm{M}_{\mathrm{a}}\right.$ \& $\left.\mathrm{S}_{\mathrm{a}}\right)$ addition of the LSC.

A sequential excitation with both $488 \mathrm{~nm}$ and $594 \mathrm{~nm}$ lasers can be used to counter the excitation crosstalk. Subtracting a constant fraction of the TexasRed signal at $594 \mathrm{~nm}$ excitation from the signal at $488 \mathrm{~nm}$ would mostly eliminate the crosstalk. However, at a ratio between FRET and crosstalk in the range of 1:10, any error in this correction has a disproportionate influence on the measured FRET signal. Since crosstalk due to direct acceptor excitation, in contrast to the FRET intensity, is not dependent on the donor concentration, this ratio is expected to further decrease with lower $P_{a p p}$. Lowering the acceptor dye concentration below $0.01 \mathrm{~mol} \%$ could increase the FRET signal relative to the direct excitation crosstalk. However, any gain in dynamic range would be negated by the increasing influence of emission crosstalk from the LSC solution. Taken together, the emission and excitation crosstalk limit the usefulness of the AF488/TexasRed FRET pair for the use case of weakly partitioning fluorphores. As it was not expected to yield any reliable results for LSCs with low $P_{a p p}$, the FRET approach was not pursued any further. 


\section{Results and Discussion}

Influence of bilayer lipid composition on lipid side chain accumulation

The influence of the bilayer lipid composition on the partitioning of the LSC conjugates was investigated by increasing the bilayer's PE content. The lipid composition of the adhered GUVs was varied from pure POPC to POPC/POPE 7:3 and POPC/POPE 4:6. At high PE contents, heterogeneous dye distribution in the bilayer of individual GUVs was observed intermittently, indicating phase separation of the membrane. Whether the separation was induced by the LSC-derivates could not be elucidated. Images showing heterogeneous membrane fluorescence were excluded from the data evaluation.

The results show that, for all three $\omega$-functionalized LSC conjugates, LSC accumulation was reduced with increasing PE headgroup content (see Figure 4.16).

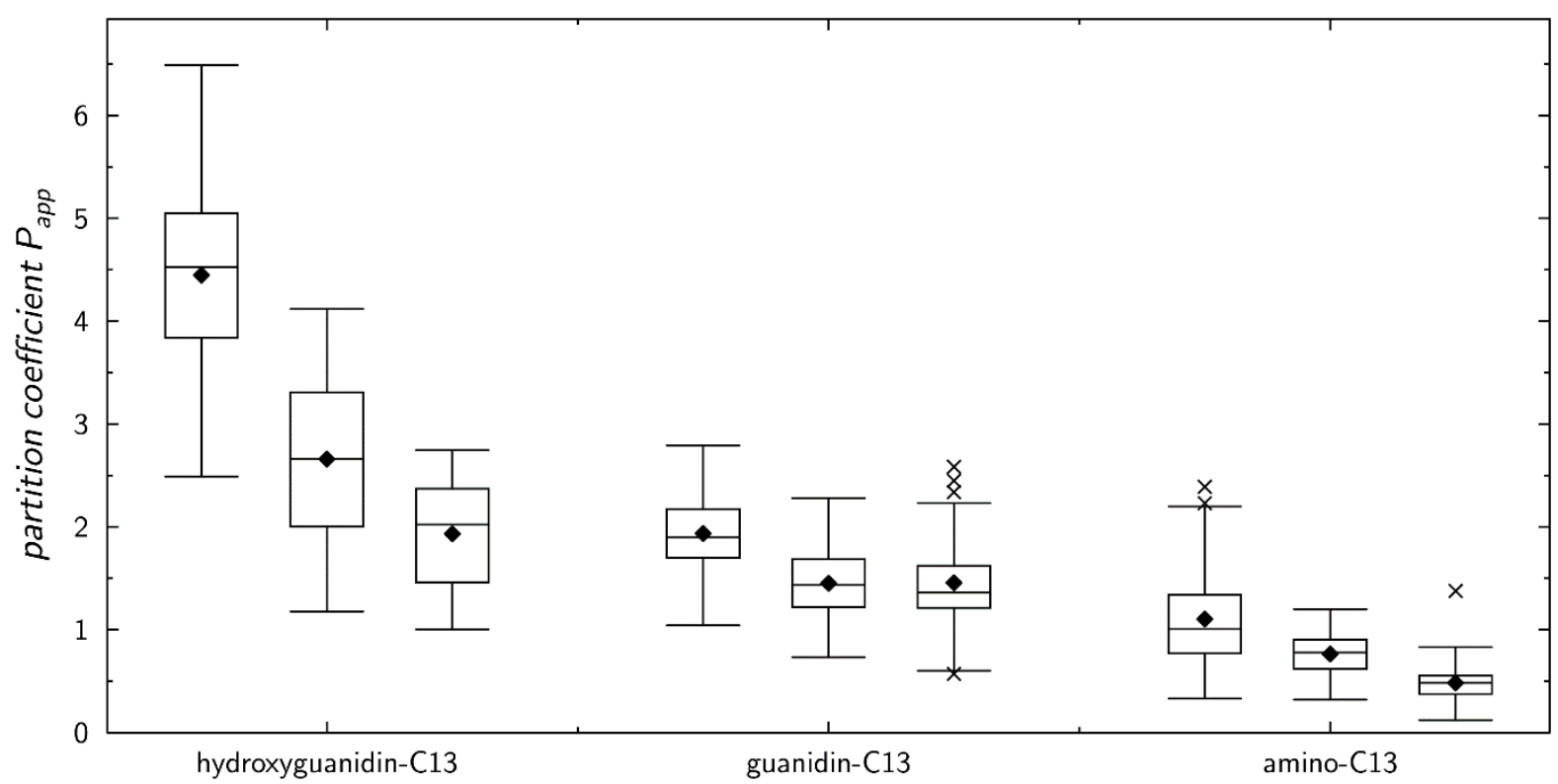

Figure 4.16 Boxplot illustrating the apparent partition coefficient $P_{a p p}$ of $\omega$-hydroxyguanidin-, guanidine- and amino-LSC conjugates in GUVs composed of POPC, POPC:POPE 7:3, and POPC:POPE 4:6 (left to right for each LSC). Each box shows upper and lower quartiles, median (line) and mean (diamond). Whiskers show the data range, except for outliers more than 1.5 times the interquartile range from the box (crosses). The medians of $P_{a p p}$ are 4.5, 2.7, $2.0(\mathrm{n}=54,90,22)$ for the A1-, 1.9, 1.4, $1.4(\mathrm{n}=90,57,63)$ for the A3-, and 1.0, 0.78, $0.48(\mathrm{n}=22,56,73)$ for the amino LSC.

The trend of decreasing $P_{a p p}$ from $\omega$-hydroxyguanidine- to amine-functionalization is not influenced by PE content but each of the functionalized LSCs shows a decrease in $P_{a p p}$ with 
PE content. The decrease is most pronounced for the $\omega$-hydroxyguanidino-LSC, with less influence of the PE content on $P_{a p p}$ for the other derivatives.

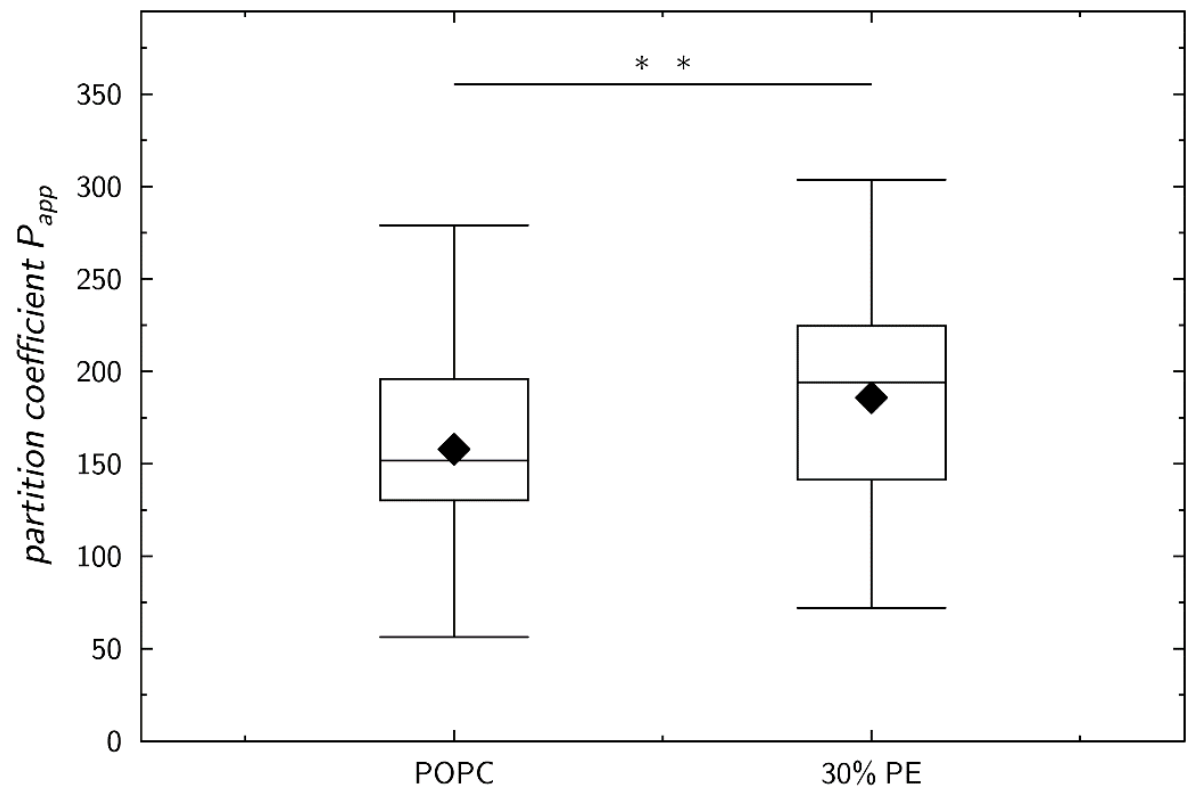

Figure 4.17 Boxplot of the apparent partition coefficient $P_{a p p}$ of unfunctionalized tridecanoic acid in GUVs composed of POPC and POPC:POPE 7:3. Each box shows upper and lower quartiles, median (line) and mean (diamond). Whiskers show the data range. The medians of $P_{a p p}$ are 152 for POPC $(\mathrm{n}=56)$ and 194 for $30 \%$ POPE $(\mathrm{n}=59)$. The difference in $P_{a p p}$ is significant $(\mathrm{P}<0.01$, Wilcoxon rank-sum test).

In contrast to the trend of decreasing partitioning observed for the $\omega$-functionalized LSCs, the unfunctionalized tridecanoic acid showed a slight increase in $P_{a p p}$ for $30 \%$ POPE compared to the pure POPC bilayer (Figure 4.17).

To quantify how varying PE content differently affected $P_{a p p}$ in the $\omega$-functionalized LSCs, the dataset was further analyzed by fitting it with a linear mixed model. The analysis was conducted in MATLAB with the standard 'fitlme' function. The model was selected according to lowest Akaike information criterion (AIC). ${ }^{[98]}$ The significance level was set at 0.05. $P_{a p p}$ was set as the response, while lipid composition, LSC derivative and their interaction were set as fixed factors. To test for confounding influences, GUV diameter, fill ratio and the width of the fitted peak were also included as fixed factors. Measurement date and GUV ID nested in measurement date were included as random factors. 


\section{Results and Discussion}

Table 2: Results for the linear mixed model for $P_{a p p}$

$\begin{array}{lrrrc} & \text { Estimate } & \text { Std. Error } & \text { t-stat } & \text { p value } \\ \text { (Intercept) } & 3.48 & 0.17 & 20.73 & <0.0001 \\ 30 \% \mathrm{PE} & -1.86 & 0.08 & -24.34 & <0.0001 \\ 60 \% \mathrm{PE} & -2.81 & 0.09 & -31.09 & <0.0001 \\ \text { guanidin-C13 } & -0.99 & 0.16 & -6.10 & <0.0001 \\ \text { amino-C13 } & -1.98 & 0.19 & -10.24 & <0.0001 \\ \text { GUV diameter } & 0.00 & 0.00 & -2.82 & <0.01 \\ \text { fill ratio } & -0.01 & 0.05 & -0.16 & 0.87 \\ \text { peak width } & -0.74 & 0.06 & -12.57 & <0.0001 \\ 30 \% \mathrm{PE}: \text { guanidin-C13 } & 1.42 & 0.10 & 14.50 & <0.0001 \\ 60 \% \mathrm{PE}: \text { guanidin-C13 } & 2.23 & 0.14 & 16.35 & <0.0001 \\ 30 \% \mathrm{PE}: \text { amino-C13 } & 1.51 & 0.11 & 13.65 & <0.0001 \\ 60 \% \mathrm{PE}: \text { amino-C13 } & 2.23 & 0.13 & 17.07 & <0.0001\end{array}$

Intercept includes the pure POPC bilayer and $\omega$-hydroxyguanidin-C13.

Sample size $\mathrm{n}=2212, \mathrm{df}=2200$.

The results of the model can be viewed in Table 2 . While the influence of fill ratio was not significant and the influence of GUV diameter was significant but negligible, peak width of the membrane fit did affect $P_{a p p}$. Using the integral of the fitted peak instead of the amplitude as a measure for $P_{a p p}$ did not reduce this influence. The results of the model were in line with the ranksum tests performed on subsets of the data, with a significant influence of basicity and PE content on $P_{a p p}$. As evidenced by their strong interaction, the type of LSC derivative decidedly determined the effect of the bilayer's PE content on $P_{a p p}$. 


\subsubsection{Discussion}

In this thesis, the interactions between lipid bilayers and several artificial analogues of the lipid sidechains of the natural antibiotic muraymycin were investigated. In a first step, the probability of fluorescently labeled lipid side chains translocating into adhered GUVs was examined. The conditions were kept similar to a previous study by Ries et al., where incubating POPC GUVs with $\omega$-hydroxyguanidino-LSC for 40 min resulted in $24 \%$ of GUVs being filled with LSC (fill ratio $\mathrm{F} / \mathrm{F}_{0}>0.5$ ), compared to only $6 \%$ in the blind experiment with AF488-acetate. ${ }^{[2]}$ This result could not be directly reproduced, as the blind experiments showed highly variable results. Passivating the avidin coated adhesion surface with BSA, an established method for suppressing unspecific binding to surfaces, ${ }^{[99]}$ did not result in more consistent fill ratios. Under certain conditions, lipids have been shown to interact strongly with BSA coated surfaces, ${ }^{[100]}$ making it less suited for the passivation of surfaces in the context of controlled lipid adhesion.

Under more stable and controlled conditions, i.e. vesicle adhesion on PEGylated surfaces, the ratios of filled vesicles for both the blind experiment and the $\omega$-functionalized LSCs were around $10 \%$ and did not differ significantly. The lipid side chain did not promote a direct translocation of the conjugated group across bilayers. Such a translocation is also not expected in the biological system, where, instead of the fluorophore, a big hydrophilic peptide-nucleoside group has to traverse the bilayer.

These results leave the possibility that the fatty acid chains destabilize lipid bilayers to some degree, just not at a level that can be quantified readily in a model system. Whether the influence of the $\omega$-hydroxyguanidino-LSC on GUV fill ratio previously measured by Ries et al. becomes apparent only under certain adhesion conditions or is due to a variability inherent in the adhesion method, remains an open question. The overall picture gained from

the experiments with LSCs in the membrane model system indicates only a minor role of membrane destabilization in the antimicrobial function of the lipid side chains. On the other hand, indirect activity mediated by the bacterial membrane could still play a role. For daptomycin, an antimicrobial lipopeptide also carrying an acyl sidechain, it has been shown 


\section{Results and Discussion}

that the interaction with the bacterial membrane can causes changes in fluidity and lipid distribution, resulting in the dislocation of MurG. ${ }^{[101]}$

The second aspect of the lipid sidechain properties investigated in this thesis was the magnitude of their accumulation in lipid bilayers. For the LSC of muraymycin A1, the $\omega$ hydroxyguanidino tridecanoic acid, accumulation had already been demonstrated in adhered GUVs, but not quantified and compared to other derivatives.[72] In this thesis, the partitioning into lipid bilayers of several LSCs with varying length and functionalization was observed via confocal microscopy and quantified by comparing the membrane fluorescence to the solution signal, with their quotient assigned as an apparent partition coefficient.

The quantitative analysis of GUV membrane fluorescence is an established method for

studying the interaction of labelled species with lipid bilayers ${ }^{[102,103]}$ The concentration is usually quantified from the fluorescence intensity in relation to calibration standards. ${ }^{[104]}$ For the very low partition coefficients studied here, this approach was not possible, as the significant absorption of excitation light in the bulk solution prohibits consistent excitation intensities between samples. Instead of to a calibration standard, the membrane fluorescence was, therefore, compared directly to the solution intensity adjacent to the vesicle. This way, variations in excitation intensity have no bearing on the results. The quotient of membrane and solution intensity is directly proportional to the actual partition coefficient of the labelled molecule and thus provides a suitable measure.

The membrane intensity is conventionally read out from a circular region of interest around the equatorial cross-section of the vesicle, maximizing the amount of image information available for analysis. ${ }^{[102]}$ In the context of low dye partitioning, this approach is not feasible due to the high background signal. To measure only the membrane signal, the mean intensity of a thin circular path along the membrane would have to be measured instead of the integral over a wide circular strip. Unfortunately, the signal to noise ratio of the images acquired from the LSC experiments was too low to ensure an accurate location of the membrane for a radial line-plot. Additionally, due to the significant influence of polarization on the membrane signal, the membrane signal can drop below the noise signal for the vertical membrane orientation. The evaluation of the complete GUV circumference would in these cases lead to a loss of information. Therefore, instead of in a circular region of interest, the 
membrane intensity was read out only from the horizontal vesicle sections, where the highest contrast to the background signal is obtained.

The membrane model system employed in this study was found to be a valuable addition to the limited portfolio of methods that allow the assessment of weak partitioning into bilayers. When studying binding of carboxylates to bilayers via SPR for example, concentrations in the millimolar range are required to obtain a signal. ${ }^{[05]}$ In the study of lipopeptides, a wide range of methods is usually employed, ${ }^{[106]}$ including zeta- potential meaurements, ${ }^{[107]}$ and bulk vesicle binding with fluorescence- or radiolabeling. ${ }^{[108]}$ The approach via confocal microscopy has the potential to expand the range of information that is accessible, e.g. the localization in phase separated systems.

The apparent partition coefficients determined from the fitted membrane intensity (see chapter 4.1.2) show several clear trends. Firstly, the partition coefficient increased with increasing end group basicity. The strong interaction of guanidine with anionic carboxylate and phosphate groups is well known, ${ }^{[109,110]}$ and the strong accumulation of guanidino-LSC in comparison to the amino derivative can be explained most readily under the assumption that the functionalized end of the fatty acid rests in the headgroup region of the lipid bilayer. Here, the specific interaction of the $\omega$-guanidine with the phospholipids can contribute to the overall energy gain of the sidechain insertion and support the accumulation. The increasing partition coefficient from the amino- to the guanidino-functionalized $\mathrm{C}_{13}$-LSCs clearly indicates an interaction between the $\omega$-end and the hydrophilic phospholipid headgroups, as an opposite trend would be expected for a location in a more hydrophobic region deeper in the bilayer.

The further increase in partitioning from the guanidino to the hydroxylated derivative mirrors the increased biological activity of muraymycin A1 in comparison to A3. No information about the interaction of $N$-hydroxyguanidino groups with phospholipids can be found in the literature, as this moiety is rarely found in membrane interacting peptides. From the clear effect it has on the partitioning, it can only be assumed that the interaction of the guanidino group with phosphate is not compromised by the hydroxy group but rather increased due to additional hydrogen bonds. 


\section{Results and Discussion}

If the functionalized chain end is located in the headgroup region of the lipid bilayer, the hydrophobic carbon chain cannot align fully with the phospholipid chains, but must instead be folded. The short functionalized $\mathrm{C}_{6}$-chains showed weak or no interaction with bilayers, whereas the longer $\mathrm{C}_{13}$-LSCs showed moderate partitioning. The unfunctionalized hexanoic acid conjugate showed some interaction, presumably because it can insert straight without folding back. Taken together, these findings indicate that the $\mathrm{C}_{13}$-chain folds into the bilayer and reaches deep enough into the hydrophobic center region to interact with it. The folded $\mathrm{C}_{13}$-chain is of comparable length to the straight hexanoic acid and a similar magnitude for the interaction with the bilayer can be assumed. The very low partitioning of the hexanoic acid derivative suggests that lipid side chains shorter than $\mathrm{C}_{13}$ should not be expected to exhibit significant accumulation when $\omega$-functionalized with a very hydrophilic group.

The low partition coefficient of the amino-functionalized $\mathrm{C}_{13}-\mathrm{LSC}$ also demonstrates that the hydrophobic interaction on its own is relatively weak and that the headgroup interaction is essential for effective accumulation. This result is in agreement with findings for acylated glycins in the range of $N_{\text {carbon }}=10-16$, where only weak binding to bilayers was found, with energies on the order of what is expected from the hydrophobic effect alone. ${ }^{[107]}$ For myristylated peptides, it has been found that electrostatic interactions in addition to the lipid insertion can greatly increase partitioning. ${ }^{[108]}$ On the other hand, the short functionalized LSCs showed no significant partitioning. This observation could be explained by the short hydrophobic loop necessarily ending up in the hydrophilic region when specific interactions of the $\omega$-functionalization are assumed. In the case of longer chains, this effect is compensated by the hydrophobic effect deeper inside the membrane, leading to a slight overall attraction.

The unfunctionalized $\mathrm{C}_{13}$-LSC showed partitioning into the lipid bilayer at least one order of magnitude stronger than the $\omega$-functionalized counterparts. This cannot result solely from the lack of solubility in the aqueous buffer, as absolute membrane concentration was estimated to be higher as well. The stronger partitioning of unfunctionalized $\mathrm{C}_{13}$-LSC can thus be attributed to its more effective interaction with the bilayer core, as the unfunctionalized carbon chain can completely insert and align with the fatty acid chains. 
Another trend apparent in the partitioning experiments was a decrease in the measured coefficients with increasing PE content of the lipid bilayer for the $\omega$-functionalized LSCs. The smaller headgroup of PE allows for a tighter packing of the unpolar lipid chains, ${ }^{[111]}$ potentially excluding the folded LSC chain from the hydrophobic region. Furthermore, the hydration state of the headgroups is influenced by the PE content: Bilayers containing PE are less hydrated in comparison to $\mathrm{PC}^{[67]}$ as the amino group of $\mathrm{PE}$ forms strong hydrogen bonds with the phosphate group. ${ }^{[12]}$ These bonds would be in competition with the interaction between the $\omega$-functionalization and the headgroups, which is assumed to involve hydrogen bonds as well. The influence of PE content on partitioning was most pronounced for the $\omega$-hydroxyguanidino-LSC. A simple exclusion of the folded chain from the core region due to packing would result in a constant effect across the different derivatives. Instead, the varying influence across derivatives indicates that the reduction in headgroup interaction is indeed the main contributor to the effect of PE content. For the unfunctionalized $\mathrm{C}_{13}$-LSC, an opposite trend was observed with slightly elevated partitioning at $30 \mathrm{~mol} \% \mathrm{PE}$ content. This finding supports the conclusion that headgroup interaction is the main factor for the reduced accumulation of the functionalized LSCs. Without this effect, the tridecanoic acid shows increased partitioning with higher PE content, either because the less hydrated headgroup region allows deeper insertion into the bilayer or because the tighter lipid chain packing allows for a more effective dispersive interaction with the inserted $\mathrm{C}_{13}$-chain.

Overall, the trend in partition coefficients observed for the differently functionalized LSCs is in line with the biological activity of the naturally occurring muraymycins. ${ }^{[41]}$ Under the assumption that no significant membrane destabilization is effected by the sidechains, these findings support the notion that the gain in antimicrobial activity of muraymycin derivatives with lipid side chains can mainly be attributed to an increased accumulation at the target membrane. Even higher activities can be expected for derivatives with stronger accumulation at membranes due to longer or differently functionalized sidechains. The main factors that likely limit the strength of the interaction in the biological context are the compounds' solubility in water and the transport out of the host cell. As shown in the tridecanoic acid, long hydrophobic sidechains severely limit solubility in water and give rise to strong binding to lipid bilayers. Under these circumstances, no simple mechanism is apparent that permits 


\section{Results and Discussion}

the transport of muraymycin to the target membrane. The $\omega$-functionalized sidechains found in vivo evidently strike a balance between water solubility and reversible interaction with the host membrane on the one hand and the accumulation of the active nucleoside antibiotic at the target organism on the other.

For the development of artificial analogs to the muraymycins, both water solubility and transport are surmountable challenges, as a wide range of drug delivery methods for similar molecules have been established. [113] A loss of specificity for bacterial cells over eukaryotes is not expected from any measure concerning the interaction with membranes, as the main mode of action is still a specific enzyme inhibition. The reduced influence of lipid composition on the accumulation of long unpolar sidechains in comparison to that of $\omega$-functionalized ones, could widen the range of organisms the substance would be active against. Moreover, if the trend of increasing MraY inhibition with increasing accumulation at the target membrane holds true for muraymycin, a significant increase in antimicrobial activity can be expected. Indeed, muraymycins with long hydrophobic anchors have been extracted from mutant Streptomyces sp. which showed good MraY inhibition and antimicrobial activity, ${ }^{[44]}$ and muraymycin derivates remain a promising topic for research. 


\subsection{Lugdunin}

The cyclic peptide lugdunin was shown to depolarize bacterial cells ${ }^{[114]}$, suggesting either disruption of the active mechanisms that maintain the electrochemical gradient across the plasma membrane, or the increase of ion permeability across the membrane. To address the question whether lugdunin can directly increase the permeability of cell membranes, a series of experiments were conducted employing large unilamellar vesicles (LUVs) as a simple and well-established model system for the bacterial plasma membrane. In a first step, the influence of Lugdunin on general membrane stability was investigated, followed by experiments to analyze ion transport across the lipid bilayer.

\subsubsection{Permeabilization of lipid bilayers by lugdunin}

A simple experiment to observe lysis of or pore formation in lipid bilayers is to monitor the release of anionic fluorescent dyes like calcein or 5(6)-carboxyfluorescein (CF) from unilamellar vesicles. These are not membrane permeable and their release can be readily observed as an increase in fluorescent signal when the dye is entrapped in a self-quenching concentration. ${ }^{[77,115]}$ Whether a gradual release or an "all or nothing" vesicle lysis is the dominating mechanism can be further investigated by requenching the fluorescence with external quenchers. ${ }^{[116]}$

To characterize the potential of lugdunin to permeabilize lipid bilayers, it was added to LUVs composed of POPC and POPC/POPG 50:50 filled with $100 \mathrm{mM} \mathrm{CF}$ at peptide to lipid ratios $(\mathrm{P} / \mathrm{L})$ of $1: 10$ to $1: 100$. As a comparison, the cyclic antimicrobial peptide Gramicidin S (GrS) was added, which has been shown to permeabilize lipid bilayers for both potassium and anionic dyes. ${ }^{[117,118]}$ The vesicles were suspended in buffer containing $275 \mathrm{mM}$ $\mathrm{KCl}$ to counter the high potassium concentration obtained in the $\mathrm{CF}$ buffer after $\mathrm{pH}$ adjustment with $\mathrm{KOH}$. This osmotic balancing resulted in a stable baseline and the addition of $1 \%$ DMSO had no influence on the fluorescence intensity except for a small dip due to dilution effects. The addition of $\mathrm{GrS}$ to the zwitterionic POPC vesicles resulted in rapid dye 


\section{Results and Discussion}

release at a high $\mathrm{P} / \mathrm{L}$ of $1: 10$ and moderate permeabilization at lower concentrations. The negatively charged vesicles showed only slow permeabilization at a $\mathrm{P} / \mathrm{L}$ of 1:10 after a short phase of rapid release (see Figure 4.18A). The lower rate seems surprising, as the cationic $\mathrm{GrS}$ is expected to interact strongly with anionic membranes and was shown to release $\mathrm{K}^{+}$ faster from vesicles containing egg PG than from those containing pure egg PC. ${ }^{[117]}$ On the other hand, a decrease in dye release for anionic lipids was observed with calcein. ${ }^{[18]}$ The sharp increase and sudden flattening of the curve could be an indication of rapid peptide insertion, faster than the mixing rate, which leads to only a small fraction of vesicles containing significant amounts of peptide. The permeabilization of further vesicles would then be limited by peptide transport, which is slow at high partition coefficients. Together, the results for GrS confirm that the CF dequenching assay utilized here is suited to give an indication whether pore formation or lysis is induced by a peptide, but can only give limited insight without employing complementary techniques. Further investigations of the permeabilization by GrS were not conducted, as the focus was on the mechanism of lugdunin activity.
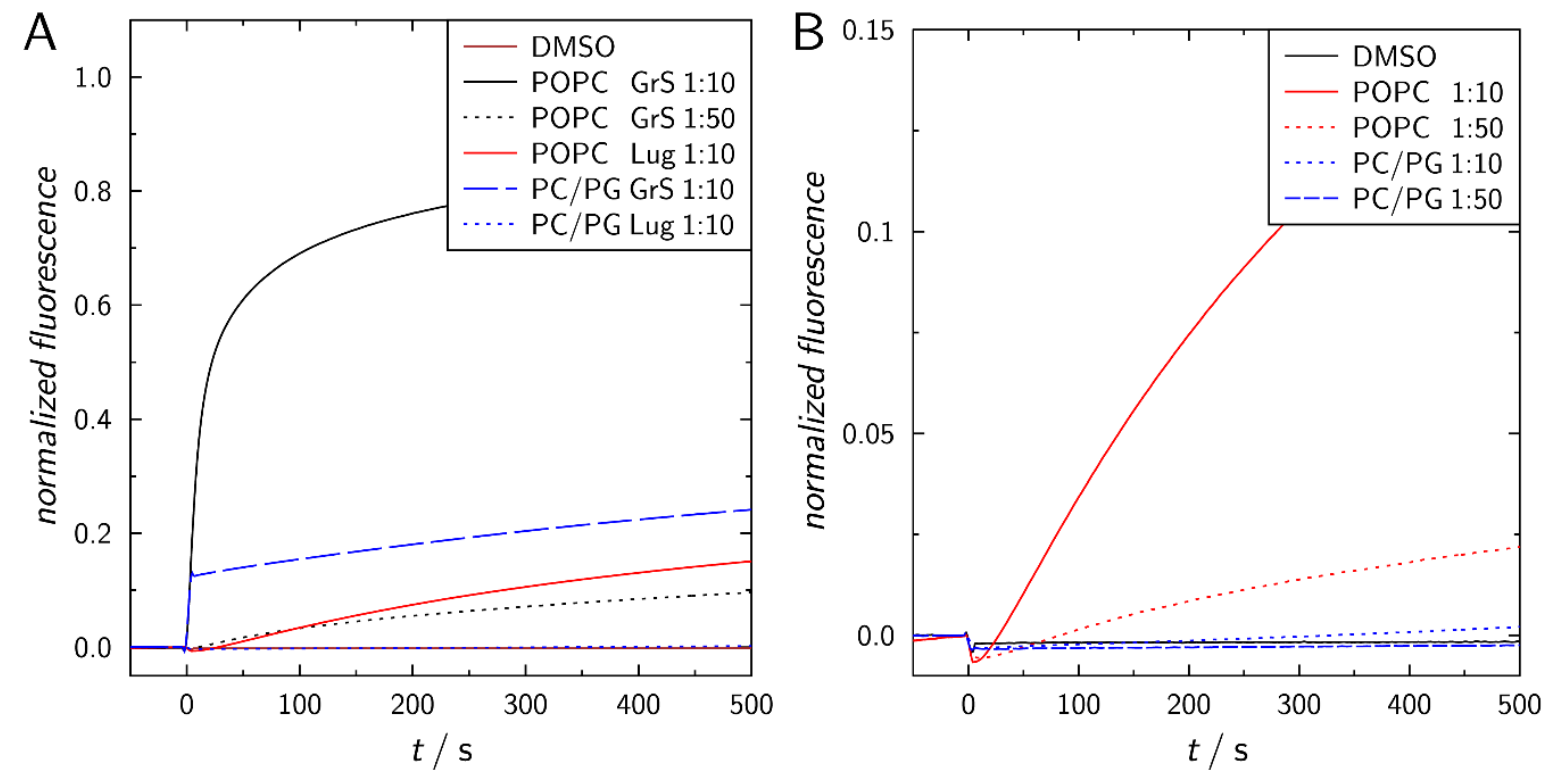

Figure 4.18: A: Time course of carboxyfluorescein leakage from LUVs composed of POPC and POPC/POPG 50:50, induced by addition of lugdunin or gramicidin S. B: Detailed view of carboxyfluorescein leakage for lugdunin additions only. Peptide dissolved in $8 \mu \mathrm{l}$ DMSO, addition at $\mathrm{t}=0$. Fluorescence intensity normalized to the intensities at $\mathrm{t}=0$ and after lysis with $16 \mu \mathrm{l}$ TritonX100 (10 wt\%). Vesicles containing $100 \mathrm{mM} \mathrm{CF}, 20 \mathrm{mM}$ HEPES, in $275 \mathrm{mM} \mathrm{KCl}, 20 \mathrm{mM}$ HEPES, $\mathrm{pH}$ 7.4, total lipid concentration $50 \mathrm{\mu M}$. 
The influence of lugdunin on bilayer permeability was significantly lower than that of gramicidin S. Dye release could be detected only with POPC vesicles at high concentrations of lugdunin. Both the initial rate of leakage as well as the fraction of free dye after a set time are comparable between lugdunin at a $\mathrm{P} / \mathrm{L}$ ratio of $1: 10$ and $\mathrm{GrS}$ at a $\mathrm{P} / \mathrm{L}$ of 1:50. No meaningful permeabilization was detected for vesicles composed of POPC/POPG 50:50 and in contrast to the measurements with GrS, no initial increase is visible in the intensity traces. This suggests that either the insertion of lugdunin into the bilayer is slower than that of GrS or that virtually no permeabilization of negatively charged bilayers is caused by lugdunin, even during initial mixing at concentrations up to 100 times above the nominal value. As the biological activity of lugdunin is generally higher than that of gramicidin $\mathrm{S}$ for many bacterial species, ${ }^{[51,119]}$ the mechanism of cell depolarization is unlikely to be governed by the relatively low potential for unspecific membrane permeabilization of lugdunin. The focus of further experiments, therefore, was moved to the investigation of specific ion transport by lugdunin across lipid bilayers.

\subsubsection{Ion Transport by lugdunin}

\section{ACMA Assay}

The first experiment investigating whether lugdunin induces proton translocation was based on a well-established acidification assay utilizing SUVs filled with potassium chloride, and the $\mathrm{pH}$-sensitive fluorescent dye 9-amino-6-chloro-2-methoxyacridine (ACMA). ${ }^{[120]}$ When the SUVs are suspended in a potassium lean buffer, an electrochemical gradient across the membrane can be generated by the conversion of the potassium concentration gradient into a transmembrane potential. This process is initiated by selectively increasing the potassium permeability with the potassium ionophore valinomycin. Vesicle acidification due to proton influx can then be detected by the quenching of ACMA fluorescence. A rapid decrease in fluorescence intensity is, therefore, expected after the addition of any proton transporter, which was confirmed in each measurement by the addition of the proton carrier carbonyl cyanide $m$-chlorophenyl hydrazone (CCCP). 


\section{Results and Discussion}

The addition of lugdunin did not, however, lead to vesicle acidification. Instead the slow baseline drift due to proton leakage was instantly stopped. Subsequent addition of CCCP still resulted in fluorescence quenching, which excludes both an interaction between lugdunin and dye as well as a complete breakdown of the electrochemical gradient as likely causes for the result. Rather, this finding indicates a compensation of the electrochemical gradient by the translocation of an ion species other than protons. For osmotic balance, the external buffer contains $\mathrm{NaCl}$, which usually does not influence the electrochemical potential, as long as the permeability for $\mathrm{Na}^{+}$is low.

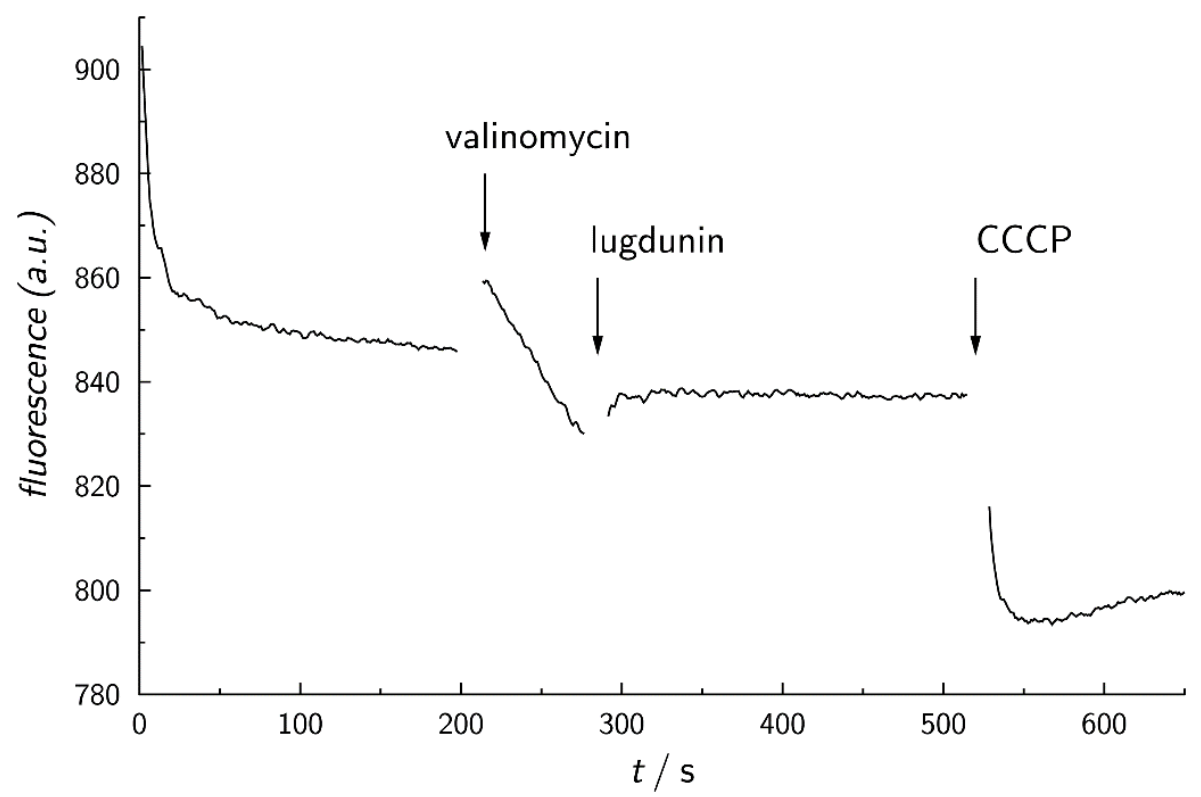

Figure 4.19: Time course of ACMA fluorescence intensity showing slow acidification of SUVs due to a negative transmembrane potential after addition of $25 \mathrm{nM}$ valinomycin. Addition of lugdunin (P/L 1:20) halts the acidification, addition of CCCP leads to acidification again. Peptides and CCCP dissolved in 8 pl DMSO, additions indicated with arrows. Vesicles composed of POPC/POPG 80:20, containing $150 \mathrm{mM} \mathrm{KCl}, 7.5 \mathrm{mM} \mathrm{NaCl}, 20 \mathrm{mM}$ HEPES in $150 \mathrm{mM} \mathrm{NaCl}, 7.5 \mathrm{mM} \mathrm{KCl} \mathrm{pH} \mathrm{7,} \mathrm{total}$ lipid concentration $100 \mu \mathrm{M}$.

To investigate whether an increase in sodium permeability was masking any proton transport by lugdunin, the experiment was repeated with an external buffer containing $150 \mathrm{mM} \mathrm{CsCl}$ instead of $\mathrm{NaCl}$. Under these conditions, the baseline after valinomycin addition did not decrease significantly, indicating a lower transmembrane potential. This can be explained by a higher permeability for $\mathrm{Cs}^{+}$in comparison to $\mathrm{Na}^{+}$, as the selectivity of valinomycin for $\mathrm{K}^{+}$ over $\mathrm{Na}^{+}$is much higher than over $\mathrm{Cs}^{+}{ }^{+}{ }^{[121]}$ Nevertheless, addition of lugdunin at peptide to lipid ratios of 1:50 and 1:100 caused acidification of the vesicles, demonstrating that lugdunin 
can indeed transport protons across lipid bilayers. Moreover, this result indicates that the flat response in $\mathrm{Na}^{+}$containing buffer is in fact caused by an increased sodium permeability due to lugdunin.

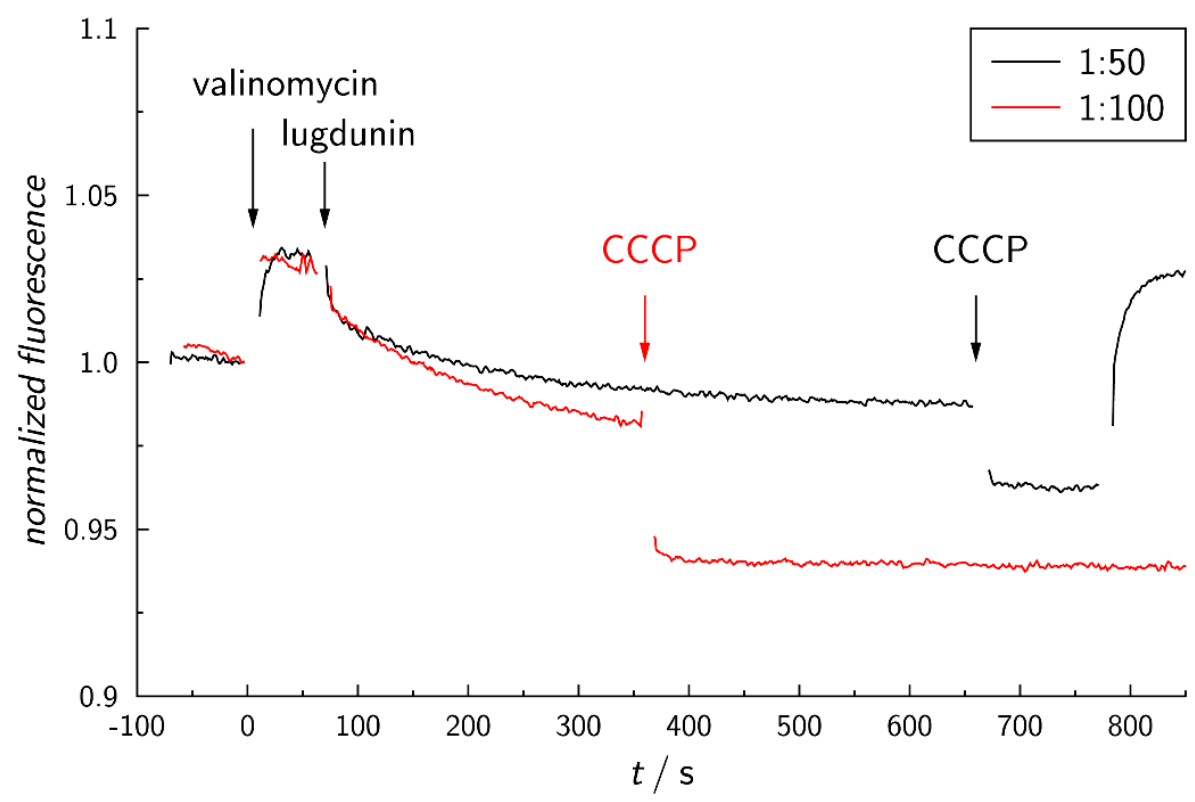

Figure 4.20: Time course of normalized ACMA fluorescence intensity showing slow acidification of SUVs after addition of lugdunin (P/L 1:50 and 1:100). Addition of CCCP leads to further acidification. Peptides and CCCP dissolved in $8 \mu \mathrm{l}$ DMSO, additions marked by arrows. Vesicles composed of POPC/POPG 80:20, containing $150 \mathrm{mM} \mathrm{KCl}, 7.5 \mathrm{mM} \mathrm{CsCl}, 20 \mathrm{mM}$ HEPES, in buffer containing $150 \mathrm{mM} \mathrm{CsCl}, 7.5 \mathrm{mM} \mathrm{KCl} \mathrm{pH} \mathrm{7,} \mathrm{total} \mathrm{lipid} \mathrm{concentration} 100 \mu \mathrm{M}$.

With the modified ACMA acidification assay it was possible to show proton transport directly and sodium transport indirectly in a qualitative way for lugdunin. Nevertheless, the assay was found to be an inadequate tool for further investigating the transport properties of lugdunin, as the indirect generation of the electrochemical gradient is only suitable for the use with selective proton transporters. Furthermore, any future efforts to compare measurements quantitatively will be complicated by the non-trivial correlation between ACMA fluorescence quenching and the actual proton gradient. Although solutions to this problem have been suggested, ${ }^{[83,122]}$ more reliable $\mathrm{pH}$-sensitive probes are available. ${ }^{[123]}$ As the investigation of antimicrobial peptides usually relies on the spontaneous insertion of peptides from aqueous solution into fully prepared vesicles, the inclusion of membrane impermeable dyes like pyranine into the unilamellar vesicles poses no challenge. 


\section{Results and Discussion}

Pyranine Assay

For the verification of lugdunin-mediated proton translocation across lipid bilayers under a wide range of peptide to lipid ratios and for different lipid compositions, a more suitable vesicle assay was utilized. The $\mathrm{pH}$-sensitive dye pyranine was entrapped into LUVs and the driving force for proton transport was switched to a direct proton gradient by changing the $\mathrm{pH}$ of the external buffer to either more acidic or more basic values. The first measurements with lugdunin showed rapid proton influx, even at much lower peptide to lipid ratios than those used in previous experiments.

To ensure that the change in pyranine fluorescence is mainly caused by proton translocation and not dye leakage, a control experiment was conducted. With POPC vesicles suspended in basic buffer, the increase in fluorescent signal due to proton efflux was monitored for different lugdunin concentrations. After $300 \mathrm{~s}$ the membrane impermeable quencher DPX was added, resulting in a drop in fluorescence intensity proportional to the absolute amount of free pyranine in solution. The measurements showed that the magnitude and concentration dependence of dye release is comparable to that of carboxyfluorescein, and is only relevant for $\mathrm{P} / \mathrm{L}$ ratios of $1: 100$ and higher.

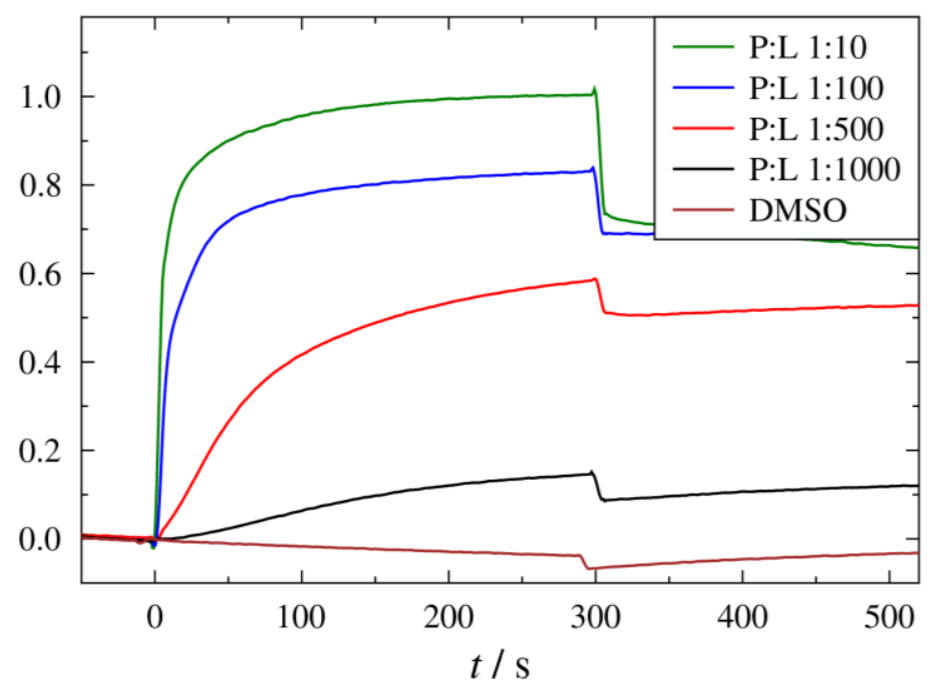

Figure 4.21 Time course of normalized pyranine fluorescence after addition of lugdunin showing proton efflux and subsequent quenching of free pyranine with $4 \mathrm{mM}$ DPX. Vesicles composed of POPC, containing $100 \mathrm{mM} \mathrm{KCl}, 5 \mathrm{mM}$ HEPES, $0.5 \mathrm{mM}$ pyranine, pH 7.4 in $100 \mathrm{mM} \mathrm{KCl}, 5 \mathrm{mM}$ HEPES, $\mathrm{pH}$ 8.4, total lipid concentration $50 \mu \mathrm{M}$. Data normalized to maximum at P/L 1:10. 
For the case of proton influx, an offset was found between the intensity at equilibrium after addition of high concentrations of peptide and the intensity after vesicle lysis (see Figure $4.22 \mathrm{~A}$ ). This shows that a sizable fraction of the dye can interact with the lipid bilayer, especially if no negative surface charge is present to repel the anionic dye. ${ }^{[124]}$ As the normalization error caused by the dye-lipid interaction is relatively small and, more importantly, constant for each lipid composition, no changes to the assay itself were made to reduce the interaction.
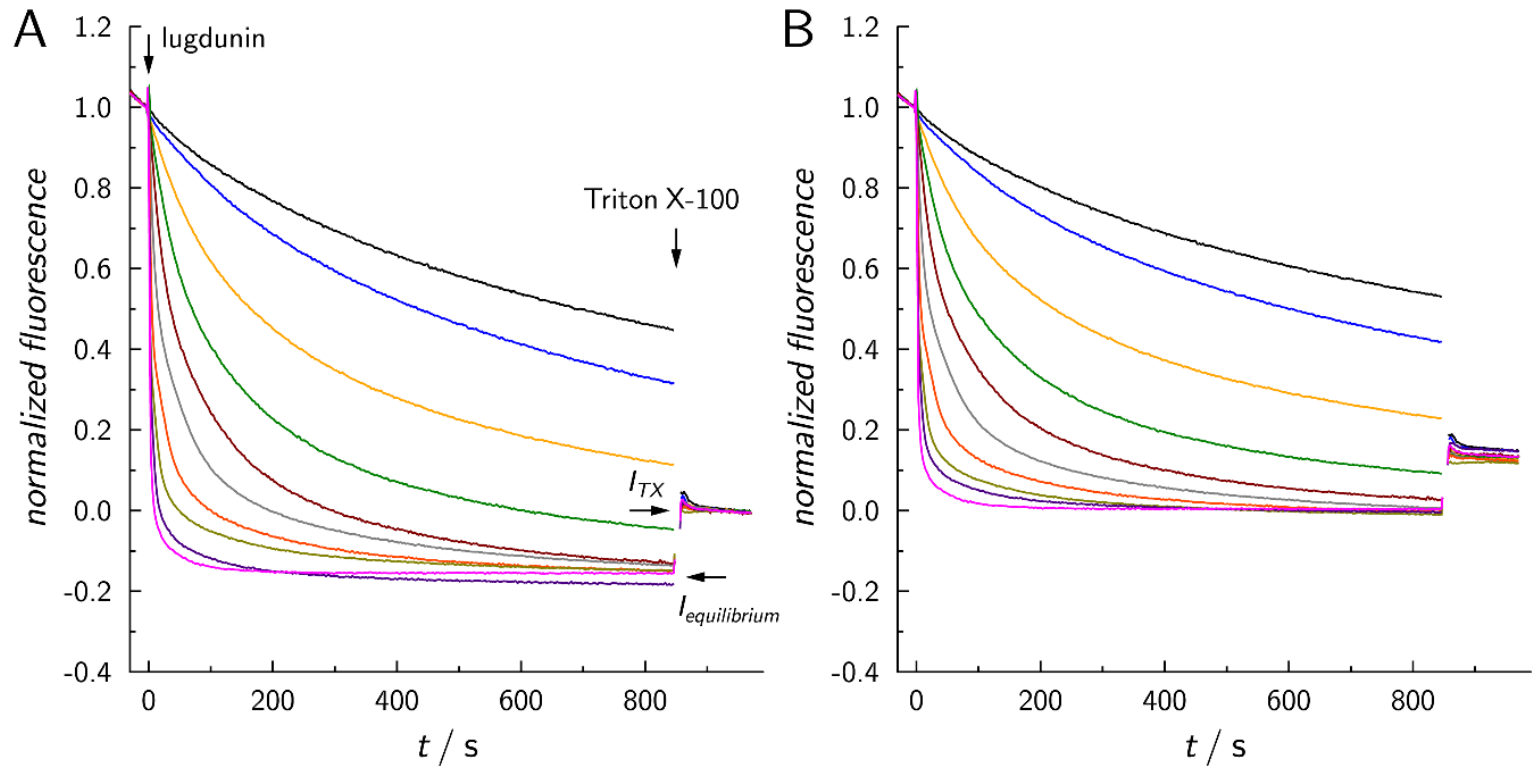

Figure 4.22: Comparison of $\mathrm{pH}$ assay fluorescence traces after uncorrected and corrected normalization. A: Data normalized to $I_{t=0}=1$ and $I_{T X}=0$. B: Normalized with correction factor $f_{d}$, which is calculated from $I_{T X} / I_{\text {equilibrium, to }} I_{t=0}=1$ and $I_{T X} / \mathrm{f}_{d}=0$.

To mitigate the significant dequenching effect of Triton X-100 on the fluorescence of pyranine enclosed in vesicles, the data normalization was extended to include a correction factor that compensates the influence of the tenside on the fluorescence intensity. For P:L ratios of 1:10 and 1:50, complete $\mathrm{pH}$ equilibrium can be assumed even before the addition of the tenside and any change in fluorescence caused by the addition can be attributed to a change in dyemembrane interaction. Utilizing this, the corrected normalization was conducted in a twostep process First, for each series of experiments with the same buffer but different peptide concentrations, a dequenching factor $f_{\mathrm{d}}$ was determined from the measurements with high lugdunin concentrations (see Figure 4.22A). 


\section{Results and Discussion}

The factor $f_{\mathrm{d}}$ is calculated as the quotient of the intensities before $\left(I_{\text {equilibrium }}\right)$ and after $\left(I_{T X}\right)$ addition of Triton X-100 (equation ( 14 )).

$$
f_{d}=\frac{I_{T X}}{I_{\text {equilibrium }}}
$$

In the second step, the data was then normalized to a virtual end intensity, given by the intensity after Triton X-100 addition $I_{T X}$, divided by the dequenching factor of the measurement series. This approach negates the dequenching and approximates the intensity that would be expected after $\mathrm{pH}$ equilibration, irrespective of any membrane-dye interaction. For the proton influx assay the intensity is decreasing and is normalized to the interval [0 1$]$ with equation ( 15$)$ :

$$
I_{\text {norm }}=\frac{I-\frac{I_{T X}}{f_{d}}}{I_{t=0}-\frac{I_{T X}}{f_{d}}}
$$

Figure 4.22 shows the direct comparison of a set of intensity traces normalized both with and without the correction factor.

For the proton efflux assay the intensity is increasing and is normalized to the interval $\left[\begin{array}{ll}0 & 1\end{array}\right]$ with equation ( 16$)$ :

$$
I_{\text {norm }}=\frac{I-I_{t=0}}{\frac{I_{T X}}{f_{d}}-I_{t=0}}
$$

The assay was conducted without explicit decoupling of proton and counter-ion flux, e.g., by addition of valinomycin. As any proton translocation along the $\mathrm{pH}$ gradient immediately generates a transmembrane potential opposing further transport, the permeability for other ions dominates the proton translocation rate. ${ }^{[125]}$ This allows a simple screening for ion permeabilities induced by the peptide. 
To this end, the proton transport was measured in buffer containing exclusively one of three cation species: $\mathrm{Na}^{+}, \mathrm{K}^{+}$, or $\mathrm{Cs}^{+}$. Both proton influx and proton efflux as a response to the addition of lugdunin were measured for the three buffers, with peptide to lipid ratios ranging from 1:10 to $1: 2000$.

The normalized intensities were plotted against time (see Figure 4.23) and the first data points were fitted with a first-degree polynomial to obtain the initial rate of change $d I / d t$.

The fluorescence intensity of pyranine is directly proportional to the $\mathrm{pH}$ around its $\mathrm{pKs}$ of $7.2^{[125]}$, near the starting $\mathrm{pH}$ of 7.4 . The initial rate of change of the $\mathrm{pH}$ can therefore be approximated by the initial rate of change in fluorescence. For a constant lipid concentration, the instantaneous rate of $\mathrm{pH}$ change at the start of each measurement can be expressed as:

$$
\frac{d I}{d t} \sim \frac{d p H}{d t}=k *[\text { Lugd }]^{m}
$$

The value was corrected for drift by subtracting the rate measured immediately before peptide addition.

From the log-log plot of the absolute value of the initial rate $d I / d t$ against the peptide concentration (see Figure $4.23 \mathrm{C}, \mathrm{F}, \mathrm{I}$ ) an approximation for the reaction order $m$ with respect to lugdunin can be extracted from the slope of a regression line through the data points. As the rates were limited at high peptide concentrations by mixing effects and the time resolution of the fluorospectrometer, measurements with P:L ratios above 1:200 were excluded from the fit. The rates for the lowest P:L ratio of 1:2000 were often near the detection limit and therefore excluded as well. 

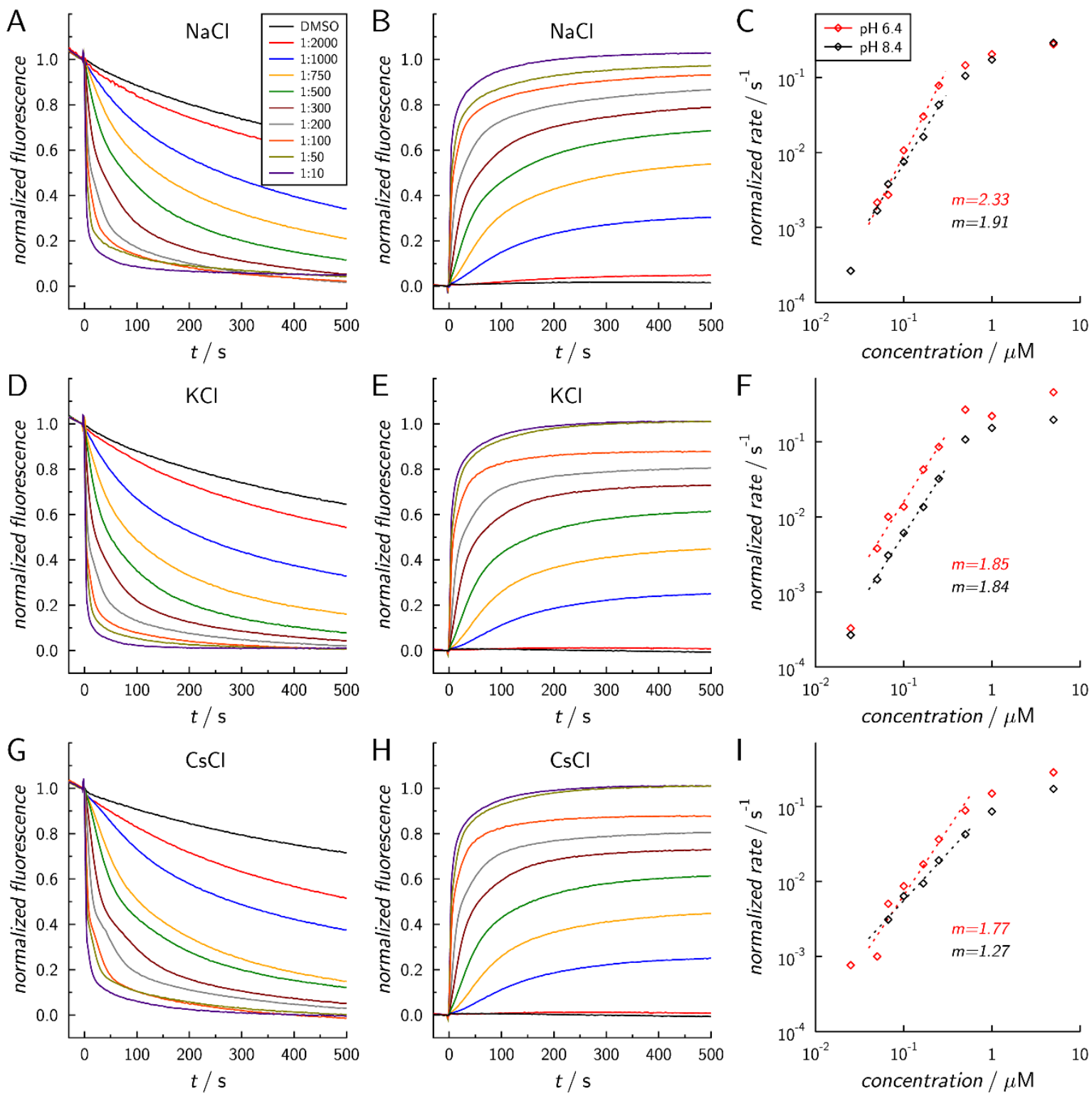

Figure 4.23: Time course of normalized pyranine fluorescence after addition of $25 \mathrm{nM}$ to $5 \mu \mathrm{M}$ Lugdunin (P:L 1:2000 to 1:10) with A,D,G: proton influx from $\mathrm{pH} 6.4$ to 7.4, B,E,H: proton efflux from $\mathrm{pH} 7.4$ to 8.4. Vesicles composed of POPC, containing $0.5 \mathrm{mM}$ pyranine in $\mathrm{NaCl}, \mathrm{KCl}$, or $\mathrm{CsCl}$ buffer. $\mathrm{C}, \mathrm{F}, \mathrm{I}$ : $\log -\log$ plot of the initial rate $\mathrm{d} I / \mathrm{dt}$, calculated from the normalized fluorescence, vs. the peptide concentration.

The observed $\mathrm{pH}$ changes after the addition of lugdunin were very similar in the three different buffers, with only slightly reduced kinetics in the case of $\mathrm{Cs}^{+}$in comparison to both $\mathrm{Na}^{+}$and $\mathrm{K}^{+}$. From the ACMA assay it was inferred that the ion conductivity induced by lugdunin is significantly lower for $\mathrm{Cs}^{+}$than for $\mathrm{K}^{+}$, which suggests that in the $\mathrm{pH}$ assay the measured proton flux is not limited by ion transport but mainly by peptide insertion kinetics. 


\section{Peptide insertion}

This notion was further examined by isolating the insertion and transport processes in the $\mathrm{pH}$ assay by switching the order of peptide addition and $\mathrm{pH}$-gradient generation. The $\mathrm{pH}-$ gradient was generated after incubation with peptide by the addition of small quantities of strong acid. ${ }^{[14]}$ The peptide was added dissolved in DMSO or isopropanol. With an acidic pulse after $300 \mathrm{~s}$ of incubation with lugdunin it was possible to observe very fast quenching of fluorescence, followed by a slow further decrease (see Figure 4.24). The response was significantly higher for peptide dissolved in isopropanol. As a control, vesicles were also prepared with pre-inserted peptide by doping the lipid solution with $0.1 \mathrm{~mol} \%$ lugdunin dissolved in isopropanol before drying. This led to instant acidification of vesicles after $\mathrm{HCl}$ addition. Longer incubation in the range of hours of POPC vesicles with lugdunin led to similar near instantaneous responses (data not shown). These observations confirm, that peptide insertion is the rate limiting step in the pyranine assay for lugdunin.

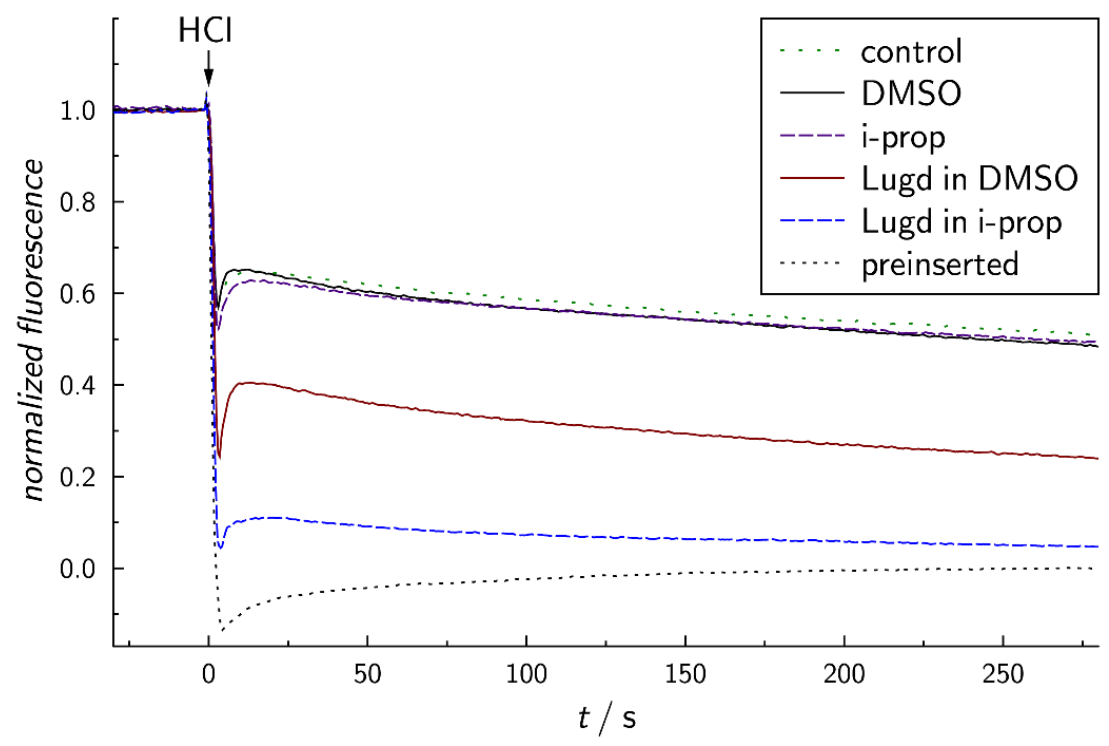

Figure 4.24: Time course of normalized pyranine fluorescence after acidification of POPC vesicles that were pre-incubated with lugdunin. $50 \mathrm{nM}$ lugdunin (P:L 1:1000) was added at $-300 \mathrm{~s}$ in DMSO or isopropanol. The last sample was measured with vesicles already prepared with $0.1 \mathrm{~mol} \%$ lugdunin in the lipid film. Vesicles containing $0.5 \mathrm{mM}$ pyranine, in $100 \mathrm{mM} \mathrm{NaCl}, 5 \mathrm{mM}$ HEPES, pH 7.4.

In order to further show if the fluorescence intensity in the $\mathrm{pH}$ assay indeed immediately represents the lugdunin insertion, an attempt was made to directly observe the peptidemembrane interaction. Lugdunin contains a tryptophan (Trp) residue and can thus be 


\section{Results and Discussion}

detected via fluorescence. Peptide insertion into membranes can often be monitored by a change in Trp-fluorescence, usually as an increase in fluorescence polarization (FP) due to the reduced mobility. ${ }^{[126]}$ Less pronounced are changes in absolute intensity and emission peak position, which are expected for Trp insertion into the hydrophobic region and stacking. ${ }^{[127]}$ A preliminary test was conducted by measuring the excitation and emission spectra of lugdunin both in buffer and after addition of POPC vesicles No clear shift in the emission peak was detected which precludes a ratiometric measurement of insertion. A clear analysis of lugdunin insertion was therefore not possible without the use of different instrumentation equipped with polarizing filters. As the HCl-pulse experiment was reproducible and clearly showed a near instantaneous ion transport in a varying fraction of vesicles, the assumption of a peptide-insertion limited response of the $\mathrm{pH}$ assay is still supported. 


\section{Influence of lipid charge}

The addition of negatively charged lipids to the bilayer had a negative effect on carboxyfluorescein leakage induced by lugdunin (see chapter 4.2.1).

In contrast to this result, ion transport, and consequently insertion of lugdunin, was found to be slightly faster in vesicles containing $50 \%$ POPG compared to pure POPC at high concentrations (see Figure 4.25). This finding contradicts the notion that slow peptide insertion could be the reason for the complete lack of unspecific lysis of POPC/POPG 50:50 vesicles. A more likely explanation is that negatively charged bilayers are not disrupted by lugdunin even at very high concentrations. At low concentrations, no significant difference was found between the insertion into bilayers composed of POPC or POPC/POPG 1:1.

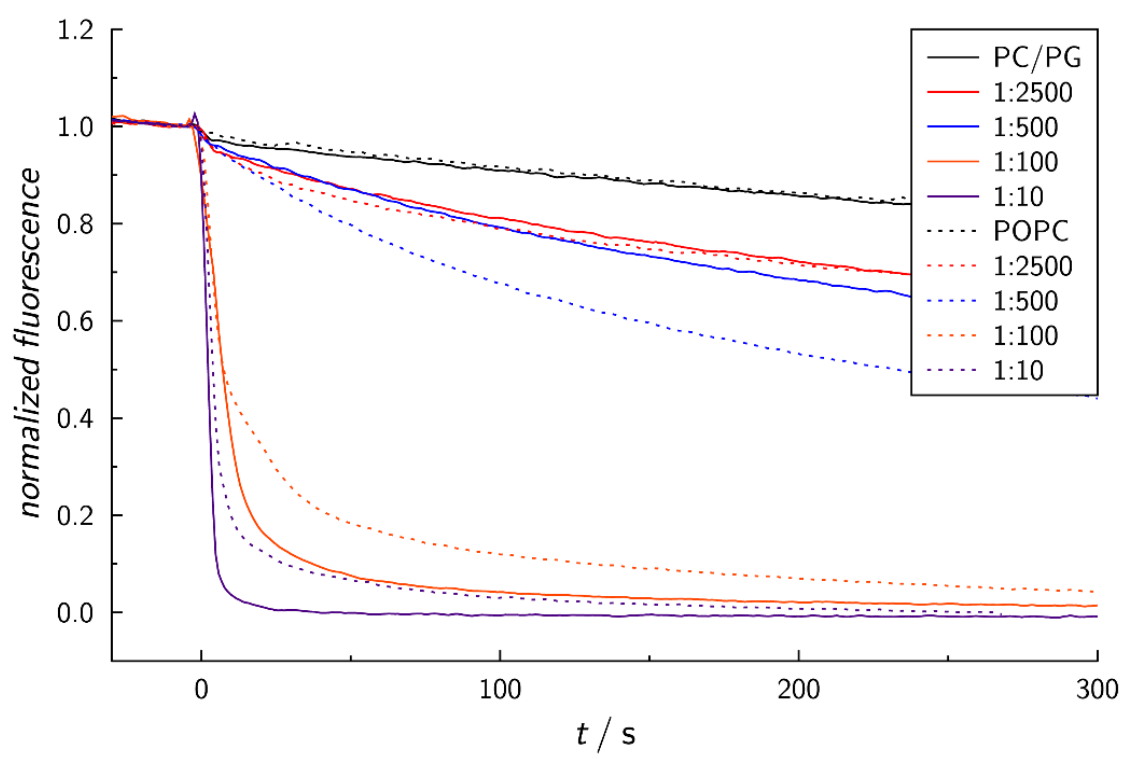

Figure 4.25: Time course of normalized pyranine fluorescence after addition of $20 \mathrm{nM}$ to $5 \mu \mathrm{M}$ Lugdunin (P:L 1:2500 to 1:10) to vesicles composed of POPC or POPG, filled with pyranine, $100 \mathrm{mM}$ $\mathrm{KCl}, 5 \mathrm{mM} \mathrm{HEPES}$ at $\mathrm{pH} 7.4$, suspended in similar buffer at $\mathrm{pH} 6.4$. 


\section{Results and Discussion}

\section{Lugdunin structure}

The N-methyl-thiazolidine derivate of lugdunin (m-lugdunin) has shown no significant antimicrobial potential in SAR studies, despite the seemingly small change in structure. ${ }^{[51]}$ Comparing the derivative's ability for ion transport to that of native lugdunin in a model system should thus prove highly informative. To this end, the ability of m-lugdunin to acidify LUVs composed of POPC and POPC/POPG 50:50 was compared to that of lugdunin. Mlugdunin showed greatly reduced membrane activity for both lipid mixtures, with significant transport only at a $\mathrm{P} / \mathrm{L}$ ratio of 1:10, where unspecific permeabilization effects are expected. Due to the low activity, no differentiation between ion transport and peptide insertion was possible for m-lugdunin.

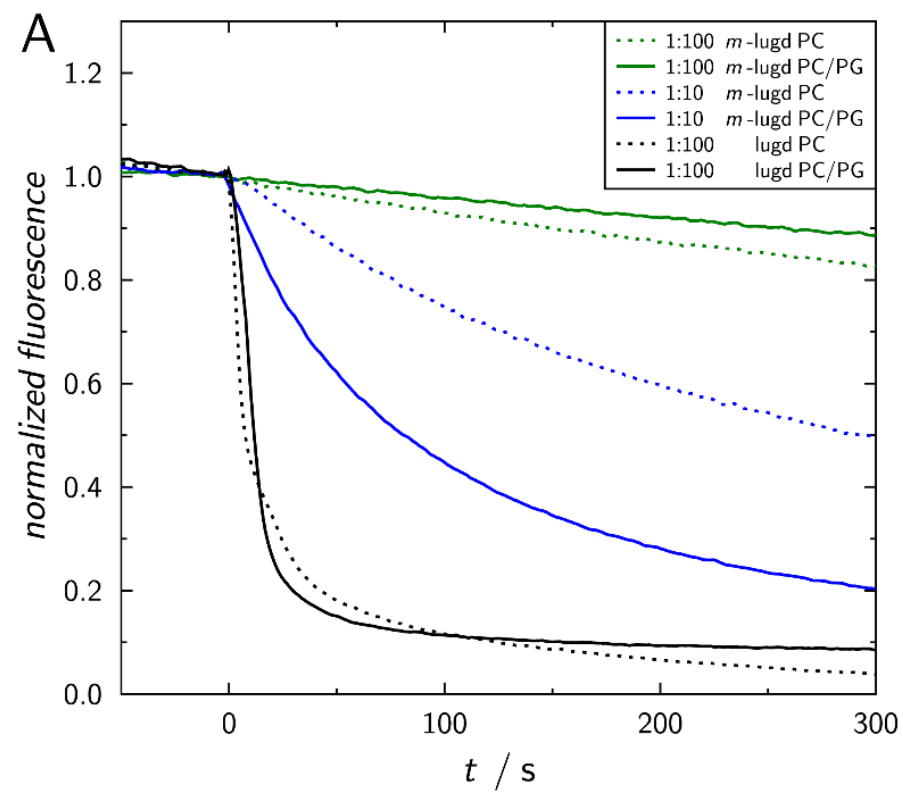

B

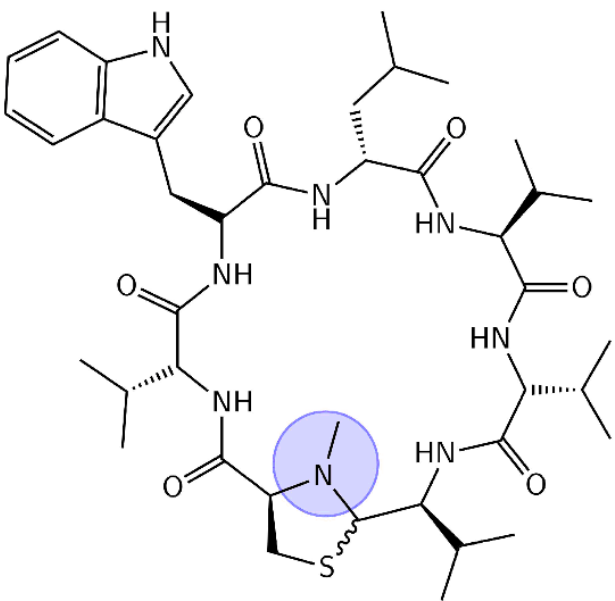

Figure 4.26 A: Time course of normalized pyranine fluorescence after addition of lugdunin (lugd) or methyl-lugdunin (m-lugd) at $\mathrm{P} / \mathrm{L}$ ratios of 1:10 to 1:100, showing proton influx. Vesicles composed of POPC or POPC/POPG 50:50, containing $0.5 \mathrm{mM}$ pyranine at $\mathrm{pH} 7.4$ in buffer at $\mathrm{pH}$ 6.4. B: Structure of m-lugdunin. The additional methyl group is marked by the colored circle. 


\subsubsection{Discussion}

The mode of action of the cyclic peptide lugdunin has been the focus of recent in-vitro studies by Schilling et al. ${ }^{[114]}$ and the experiments in this thesis were designed to both verify those findings in a model system and highlight further routes worth investigating. The vesicle experiments presented here complement the assays conducted with S.aureus and the results of both investigative approaches are in good agreement overall. Specifically, in chapter 4.2.1 it was found that lugdunin only has a weak influence on bilayer stability. Vesicles filled with carboxyfluorescein showed no or very slow dye leakage at peptide to lipid ratios of up to 1:10. This finding mirrors the lack of membrane permeabilization observed in S.aureus after addition of lugdunin, indicated by the exclusion of the red fluorescent dye propidium iodide from cells, ${ }^{[114]}$ and confirms that large pores or membrane disruptions are not the main mode of action for lugdunin. Further experiments, using the pH-sensitive dye pyranine to monitor ion transport across lipid bilayers, showed that lugdunin can induce permeability for protons and monovalent cations at peptide to lipid ratios of 1:2000 and lower. This finding substantiates the results from depolarization experiments with the membrane potential sensitive dye $\mathrm{DiOC}_{2}(3)$ performed by Schilling et al. and clearly shows that the observed depolarization can be attributed to ion transport across the plasma membrane. ${ }^{[14]}$

In summary, the results of this thesis show that the bactericidal activity of lugdunin can be ascribed to an increase in membrane permeability for ions, which in turn disrupts the transmembrane potential that is essential for ATP production.

\section{Selectivity}

In addition to investigating the general mode of action of lugdunin, the experiments employed in this thesis allow further insights into the interaction between lugdunin and specific lipid bilayers. One topic of interest in this regard is the question of selectivity. The high amount of anionic lipids like PG or cardiolipin in bacterial membranes is considered an important factor for the selectivity of many antimicrobial peptides ${ }^{[128]}$ and can be readily

modeled with anionic lipid mixtures in permeabilization and binding assays. ${ }^{[129]}$ Besides 


\section{Results and Discussion}

charge, the presence of specific headgroups may also be required for the interaction with some peptides. For example, for the cyclic lipopetide daptomycin, a clear dependency of cation transport on the presence of PG has been shown in a model system similar to the one employed here, whereas the presence of similarly charged cardiolipin did not promote peptide activity. ${ }^{[130]}$

When comparing the depolarizing effect of lugdunin on neutral and anionic lipid bilayers, only small differences were observed between measurements with vesicles composed of POPC and POPC/POPG. This apparent non-selectivity stands in stark contrast to the findings in live cells by Zipperer et al., who found low cytotoxicity of lugdunin at concentrations up to $50 \mathrm{\mu g} / \mathrm{ml}$, which suggests a high selectivity for bacterial cells. ${ }^{[51]}$ The magnitude of ion transport across lipid bilayers observed both in the model system and in cells seems to be sufficiently high to explain the antimicrobial action on its own. However, unspecific transport of monovalent cations across bilayers is usually linked to high toxicity for eukaryotic cells as well, as seen for example with the ionophore gramicidin A.[131-134] Therefore, the most likely cause for the selectivity should indeed be sought in differences in the interaction between lugdunin and membranes of varying type. The high activity observed in the minimalistic POPC model membrane suggests that the insertion and transport ability of lugdunin is not dependent on specific properties of the bacterial membrane. It can, therefore, be assumed that the activity against eukaryotic cells is extenuated by an unknown mechanism. Further studies on lugdunin are thus warranted, both to gain general insights into the source of the selectivity as well as to identify specific pathways that could lead to resistances against antibiotics derived from lugdunin and similar peptides. In a first step, the lipid mixture of the model system could be further varied. In particular, the influence of the cholesterol content on lugdunin activity would be of interest, as its presence in eukaryotic membranes is a fundamental differentiating factor. ${ }^{[118]}$ 


\section{Mechanism}

In concurrence with the question about selectivity, the exact ion transport mechanism of lugdunin needs to be elucidated. This thesis took a first step towards this goal by examining the ion transport rates measured for different peptide concentrations.

The initial decrease in fluorescence does not always follow a simple exponential curve. At peptide to lipid ratios in the range of 1:100 a discontinuity in the traces indicates the influence of two different processes (see Figure 4.23). Whether this is a mixing effect or a sequential process of insertion and aggregation of lugdunin with rate constants in a similar range is not clear from the data. Nevertheless, at low concentrations ( $\mathrm{P}: \mathrm{L}<1: 100)$, the reaction order with respect to lugdunin is significantly higher than 1 , which suggests that more than one molecule is involved in ion transport and some form of oligomerization takes place.

The amino acids of the native lugdunin are configured in alternating D- and L-configurations and the biological activity has been shown to be strictly dependent on this alternating pattern, regardless of the overall absolute configuration. ${ }^{[114]}$ Cyclic peptides with an even number of amino acids and strict DL-alternation are known to self-assemble into stacks in lipid bilayers and span the hydrophobic core with a cylindrical $\beta$-sheet-like, hydrogen-bonded peptide structure.[135-140] Selective transport of alkali metals, without much selectivity between the elements in the series from $\mathrm{Na}^{+}$to $\mathrm{Cs}^{+}$, has been observed in such peptide nanopores, ${ }^{[141]}$ and high single channel conductance values with characteristic gating has been measured..110] Antimicrobial activity of cyclic DL-octapeptides has been shown against E.coli and MRSA. Here, activity and specificity where significantly influenced by the amino acid sequence. ${ }^{[142]}$ Lugdunin is less symmetric than these structures due to the thiazolidine moiety, but stacking of DL-peptides containing a ring linker has been observed. ${ }^{[138]} \mathrm{A}$ similar stacking of lugdunin to form a continuous pore is the most likely mechanism for its oligomerization and should be further investigated.

Whether the $\mathrm{N}$-methylated thiazolidine derivate did not insert into the bilayer or inserted without forming pores could not be answered from the limited set of experiments conducted. 


\section{Results and Discussion}

In either case, the stark difference in activity mirrors the results from cell assays ${ }^{[14]}$ and accentuates the important role of the thiazolidine ring in lugdunin.

The influence of the solvent on the insertion rate observed in the HCl-pulse experiments suggests that lugdunin can assume at least two distinct conformations in solution, which has been described for many cyclic peptides. ${ }^{[143-146]}$ Two main conformations can be expected, one where the hydrophobic amino acid residues are oriented towards the center of the macrocycle, and one where residues are exposed to the surrounding solution and intramolecular hydrogen bonds can be formed between amides. ${ }^{[47]}$ Partitioning of lugdunin into the hydrophobic core of the bilayer is most likely reserved to the latter case. The stabilization of a conformation favoring the aqueous solution by DMSO could explain the observed differences in insertion rate. Alternatively, a faster equilibrium between conformations in isopropanol would also result in a difference. The unique thiazolidine ring in lugdunin could greatly enhance the rate of conformational changes in comparison to classical cyclopeptides. If m-lugdunin is found to not insert into bilayers, a hindrance by the methyl group of the conformational change necessary for insertion into the bilayer seems likely. 


\section{$4.3 \quad \mathrm{AAO}$}

The specificity of AMP action towards prokaryotic cells is a crucial characteristic in the development of antibiotic drugs based on these peptides. One important aspect is the dependence of permeabilization on membrane composition. Besides charge, the lack of cholesterol in prokaryotic membranes is a prominent distinction from eukaryotic plasma membranes. Aptly, the cholesterol content of lipid bilayers has been shown to influence the ability of AMPs to permeabilize membranes. ${ }^{[148,149]}$ While cholesterol content generally suppresses permeabilization by AMPs, the opposite effect can also be found in a heterogeneous, phase-separated membrane model system. The peptide $\delta$-lysin, for example, shows high partitioning into the liquid-disordered $\left(l_{d}\right)$ phase in vesicles composed of the ternary mixture of sphingomyelin, DOPC, and cholesterol. This localization of peptide in turn increases the permeabilization rate in comparison to simple cholesterol-free membranes. ${ }^{[150]}$ It is generally believed, that cell membranes exhibit heterogeneous lipid composition and are separated into transient nano-domains, the so-called lipid rafts. ${ }^{[151]}$ The spreading of phase-separated vesicles on a planar porous substrate would allow the simultaneous assessment of membrane permeabilization for both phases of a given lipid mixture. This method would complement existing techniques and further the understanding of peptide interactions with heterogeneous membranes. In this thesis, it was thus attempted to establish a process for this method.

In a previous study, phase-separated GUVs composed of sphingomyelin/DOPC/cholesterol in a molar ratio of 2:2:1 were spread successfully on functionalized AAO. ${ }^{[62]}$ At temperatures below approx. $40{ }^{\circ} \mathrm{C}$ this ternary mixture separates into a liquid ordered $\left(l_{o}\right)$ phase, rich in sphingomyelin and cholesterol, and a liquid disordered $\left(l_{d}\right)$ phase, composed mainly of the unsaturated DOPC.[70] At first, it proofed difficult to spread phase-separated GUVs on porous alumina at room temperature without a rapid loss of phase separation in the individual pore spanning membrane patches. Only when spreading at a temperature of $4{ }^{\circ} \mathrm{C}$, a high yield of membrane patches that still showed clear phase separation was obtained. These pore-spanning membranes were shown to be stable and retained phase separation for hours when slowly equilibrated to room temperature during microscopy. When incubated 


\section{Results and Discussion}

with magainin-2, the rate of membrane permeabilization showed a clear difference between the $l_{o}$ and $l_{d}$ phases. ${ }^{[62]}$ These results demonstrate that the model system of planar pore spanning membranes could be a promising tool for the investigation of peptide specificity towards lipid bilayers with a heterogeneous composition.

\subsubsection{Permeabilization assay on phase-separated lipid bilayers}

One objective of this thesis was to further investigate the phase-separated permeabilization assay and ascertain the feasibility of expanding it by incorporation of nanowires into the substrate. A first step towards this goal was the reproduction of the previous experiments and the identification of potential issues in the interplay between the spreading of phaseseparated membranes and a modified substrate. To this end, phase-separated GUVs composed of sphingomyelin/DOPC/cholesterol in a molar ratio of 2:2:1, doped with TexasRed-DHPE and BODIPY-cholesterol (0.25 mol\% each) were prepared by electroformation (see chapter 3.1.3). The lipid mixture was doped with two fluorescence markers to enable the reliable detection of $l_{o}$ phase domains. The headgroup-labeled TexasRed-DHPE distributes into the $l_{d}$-phase nearly exclusively, while the dye BODIPYcholesterol shows a slight preference for the $l_{o}$-phase. For spreading, orthogonally functionalized AAO substrates were immersed in precooled PBS buffer, incubated with vesicles for $10 \mathrm{~min}$, and gently rinsed at $4^{\circ} \mathrm{C}$. The membrane patches obtained from vesicle spreading were subsequently imaged via confocal fluorescence microscopy. 

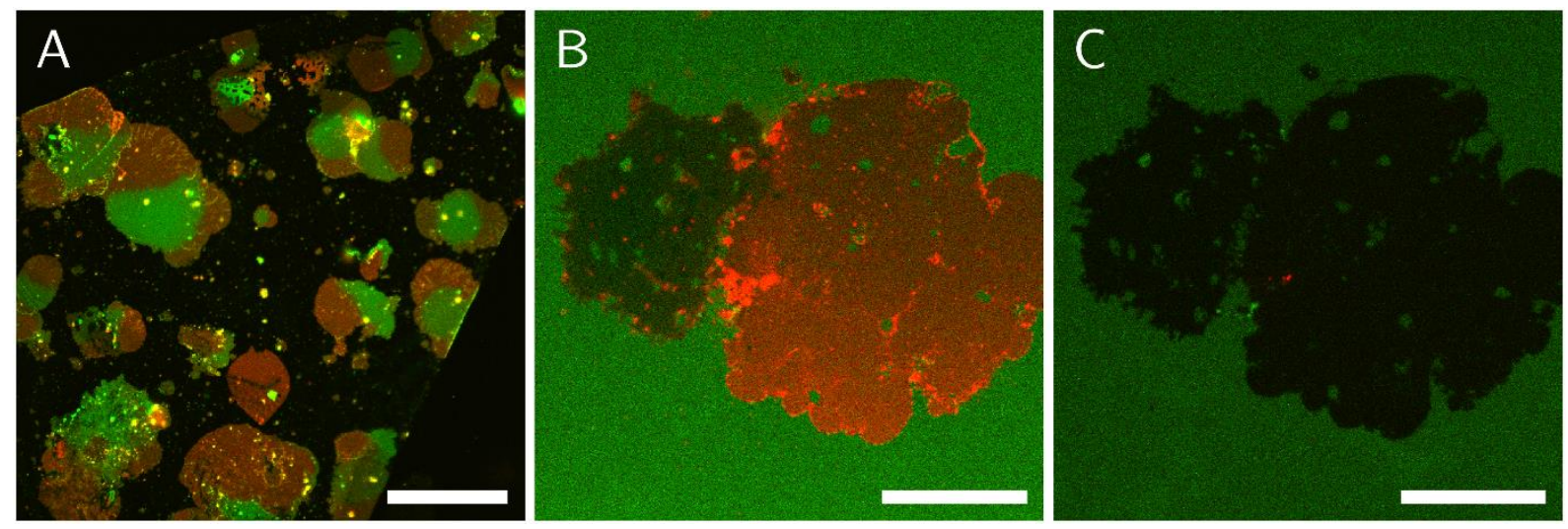

Figure 4.27: Confocal fluorescence micrographs of phase-separated pore-spanning bilayers on porous alumina. A: Overview of patches obtained from spreading GUVs composed of sphingomyelin/DOPC/cholesterol in a molar ratio of 2:2:1, doped with TexasRed-DHPE (red channel) and Bodipy-cholesterol (green channel) at $4{ }^{\circ} \mathrm{C}$, scale bar $50 \mu \mathrm{m}$. B: Individual phase-separated patch after addition of $1 \mathrm{mM}$ pyranine to the supernatant, imaged in the plane of the substrate surface. $l_{0^{-}}$ marker fluorescence is masked by pyranine. C: Focal plane $5 \mu \mathrm{m}$ below the substrate surface, showing pyranine exclusion from the porous substrate beneath both phases, scale bar $20 \mu \mathrm{m}$.

Directly after spreading, planar pore-spanning membrane patches with clearly distinguished phase domains were found (Figure 4.27A). Both phases showed good pore sealing, as evidenced by the exclusion of fluorescent dye in the aqueous solution from the substrate pores (see Figure $4.27 \mathrm{C}$ ). In most cases, continuous patches with one larger $l_{d}$ and one smaller $l_{\sigma^{-}}$domain were obtained, surrounded by a varying amount of excess lipid material from vesicle rupture. This structure directly mirrors the phase distribution in GUVs, were two large domains are favored due to the line tension at the phase boundaries. ${ }^{[152]}$ While some patches maintained a stable phase separation even after warming to room temperature under the microscope, most showed a gradual loss of the clear boundary between $l_{\sigma^{-}}$and $l_{d^{-}}$ phase with time. The marker dye migration was mainly observed as TexasRed-DHPE diffusing into the $l_{\sigma}$-region (see Figure 4.28). The rate and extent of this apparent lipid redistribution were highly variable both between individual patches and between preparations. 

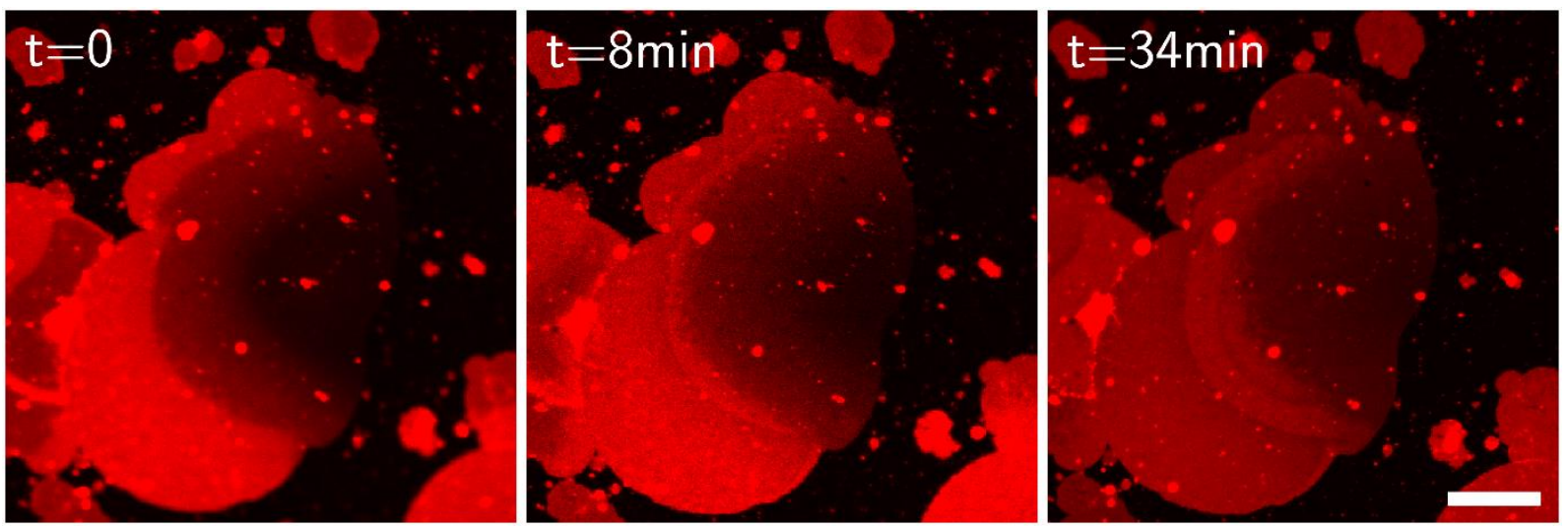

Figure 4.28: Fluorescence micrographs of a phase-separated pore-spanning membrane patch on porous alumina after warming to room temperature. At the start of the observation, the $l_{0}$-region shows TexasRed-DHPE fluorescence in the vicinity of the phase boundary. In the course of minutes, the $l_{d^{-}}$ marker dye is observed to further diffuse into the $l_{0}$-region. Scale bar $20 \mu \mathrm{m}$.

As temperature was expected to have a significant influence on lipid diffusion, simply letting the substrate warm to room temperature was unsuitable for a controlled study of the phenomenon. Due to the high thermal mass and unknown thermal conductivity of the water immersion objective, small variations in microscopy procedures have unpredictable effects on the temperature development of the sample. Therefore, an all-metal sample holder was designed that allows the precise control of substrate temperature. A custom microscope stage was built to cool or heat the sample holder via a Peltier element (see chapter 3.5.4).

In a first experiment, phase separated GUVs were spread on AAO at $0{ }^{\circ} \mathrm{C}$ directly on the microscope stage and subsequently warmed at a rate of $0.1{ }^{\circ} \mathrm{C}$ per second. At $5{ }^{\circ} \mathrm{C}$ intervals the ramp was paused for several minutes. The substrate was imaged continuously during the experiment via fluorescence microscopy. Slow changes in dye distribution were observed in one sample even at $0{ }^{\circ} \mathrm{C}$, while significant mixing was usually observed at temperatures above $15{ }^{\circ} \mathrm{C}$. 

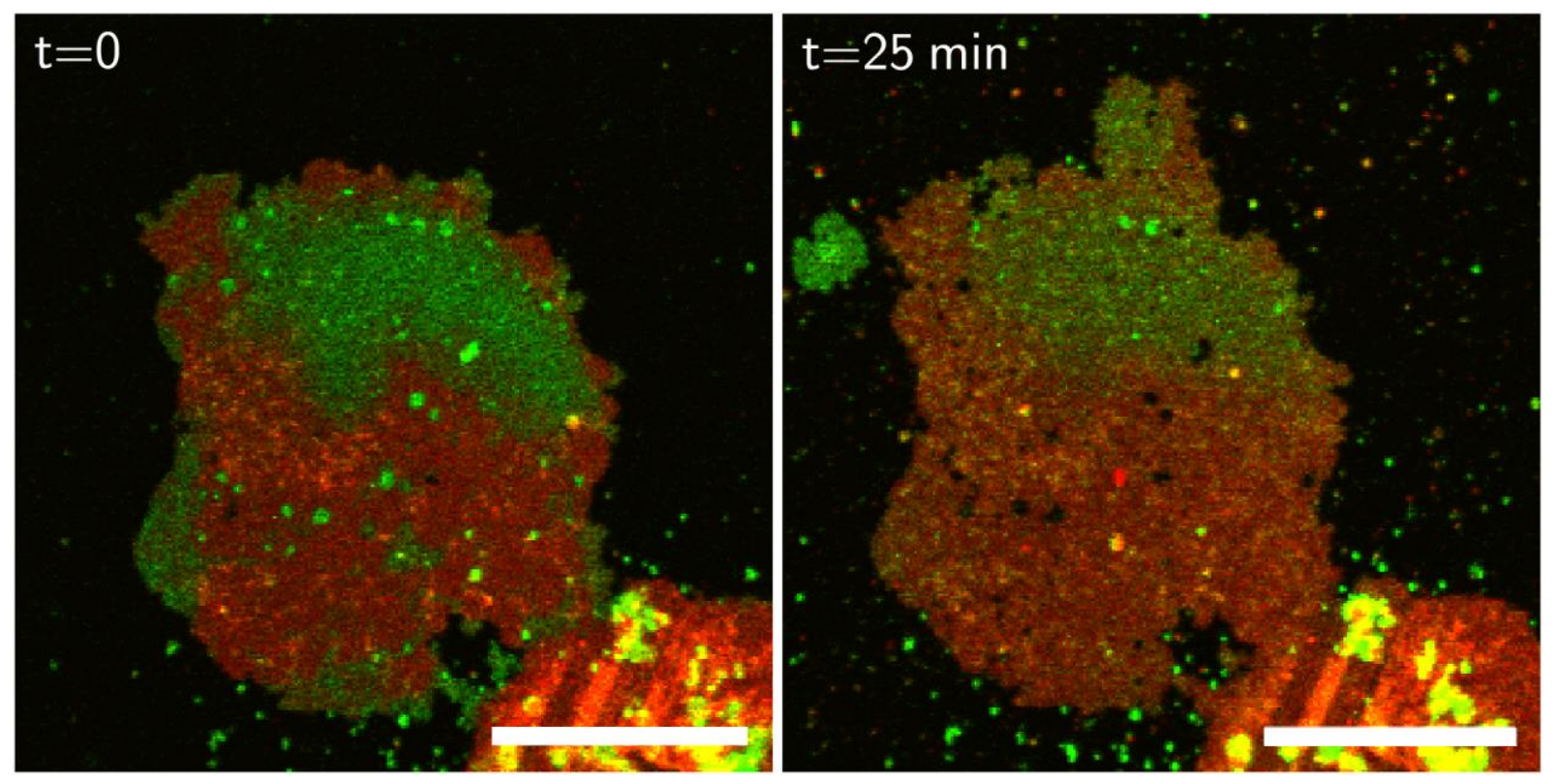

Figure 4.29: Fluorescence micrographs of a phase-separated pore-spanning membrane patch on porous alumina after spreading at $0{ }^{\circ} \mathrm{C}$. The sample was held at $0{ }^{\circ} \mathrm{C}$ for $25 \mathrm{~min}$. During this period significant diffusion of $l_{d}$-marker into the $l_{o}$ region is observable. Scale bars $20 \mu \mathrm{m}$.

On one of the samples, mean membrane fluidity was determined via fluorescence recovery after photobleaching (FRAP) measurements at temperatures between $0{ }^{\circ} \mathrm{C}$ and $20{ }^{\circ} \mathrm{C}$ in $5{ }^{\circ} \mathrm{C}$ steps. Bleaching was performed on both $l_{d}$ and $l_{o}$-phase regions of several patches. The diffusion coefficient increased approximately by a factor of 4 for the $l_{d}$-phase and a factor of 10 for the $l_{\sigma}$-phase in the temperature interval from $0{ }^{\circ} \mathrm{C}$ to $20{ }^{\circ} \mathrm{C}$. 


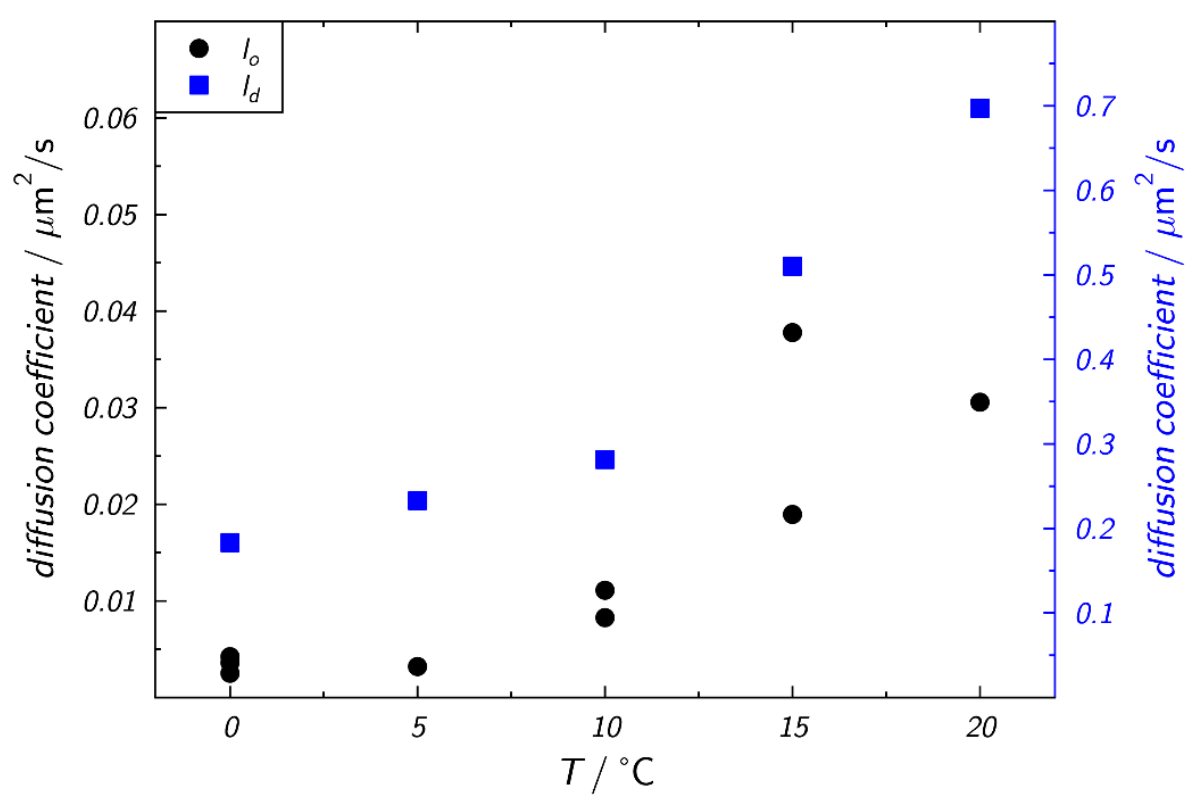

Figure 4.30: Diffusion coefficients from FRAP measurements for the $l_{d}$-phase (blue) and the $l_{o}$-phase (black) patch on porous alumina at temperatures between $0{ }^{\circ} \mathrm{C}$ and $20{ }^{\circ} \mathrm{C}$.

Together, these observations show that the $l_{0}$-phase is in a state of very low fluidity at low temperatures and significant redistribution effects only begin above $15{ }^{\circ} \mathrm{C}$. This partly explains the variability in previous experiments concerning the loss of the phase boundary, as temperature would have a highly nonlinear influence on the rate of mixing. However, the varying mixing rates of different samples at a controlled low temperature suggest that variations in the interaction between bilayer and substrate also influence the redistribution.

In order to directly observe the mechanisms involved in the lipid mixing of planar bilayers on porous support structures, the behavior of phase separated membranes has to be studied on a substrate with pore sizes above the diffraction limit. A silicon nitride substrate with a hexagonal pattern of pores $450 \mathrm{~nm}$ in diameter (micro sieve plate, $30 \%$ porosity, fluXXion B.V., Eindhoven, The Netherlands) was selected as an analogue to the AAO scaffold. As lipid material readily spreads on bare silicon nitride after plasma cleaning, the functionalization employed on AAO cannot be directly transferred to the $\mathrm{Si}_{3} \mathrm{~N}_{4}$ substrate. Without a differentiation between pore walls and the upper rims, the pore interiors would be coated with lipid material during GUV spreading. Therefore, a modified orthogonal functionalization was developed that mimics the non-silanized hydrophilic pore interior and the silanol terminated pore rims of functionalized AAO. The $\mathrm{Si}_{3} \mathrm{~N}_{4}$ substrates were first 
oxidized with $\mathrm{O}_{2}$-plasma, followed by the evaporation of $30 \mathrm{~nm}$ of gold onto the pore rims. The pore interiors were subsequently silanized with APTES and passivated with PEG chains by reacting the exposed surface amino groups with NHS-PEG (see chapter 3.5.3). Two different methods were employed to remove the gold mask after PEGylation. First, as in the case of masked AAO, the gold layer was removed chemically with an aqueous $\mathrm{KI} / \mathrm{I}_{2}$ solution. Without a further step of $\mathrm{O}_{2}$-plasma treatment, which was suspected to deteriorate the PEG passivation layer, this method yielded mostly adhered, but very few completely spread vesicles. A short pulse of plasma treatment in an $\mathrm{H}_{2} \mathrm{O}$ atmosphere $\left(\mathrm{t}=1 \mathrm{~s}, 10\right.$ mbar $\left.\mathrm{H}_{2} \mathrm{O}\right)$ after the chemical gold removal enabled the spreading of vesicles, but pore-spanning membranes were found to be unstable with time. In contrast to the first method, the mechanical removal of the gold mask with adhesive tape resulted in a surface that showed similar spreading activity as orthogonally functionalized AAO. Large patches of stable porespanning membranes were obtained when spreading phase separated GUVs. The fluorescence intensity on the pore rims was difficult to assess, as it is partially quenched by the underlying substrate. Therefore, phase separation could only be evaluated on the freestanding membranes for some samples.

Directly after vesicle spreading, clear phase separation was found, even when spreading at $20{ }^{\circ} \mathrm{C}$. With time, a rearrangement of the marker dyes was observed, evidenced by a stepwise replacement of $l_{d}$-phase by $l_{o}$-phase on the freestanding membrane parts at the boundary between both regions (see Figure 4.31). Each visible rearrangement process was not instantaneous however, as a phase boundary can be identified on individual pores in some of the micrographs obtained during the observation time.
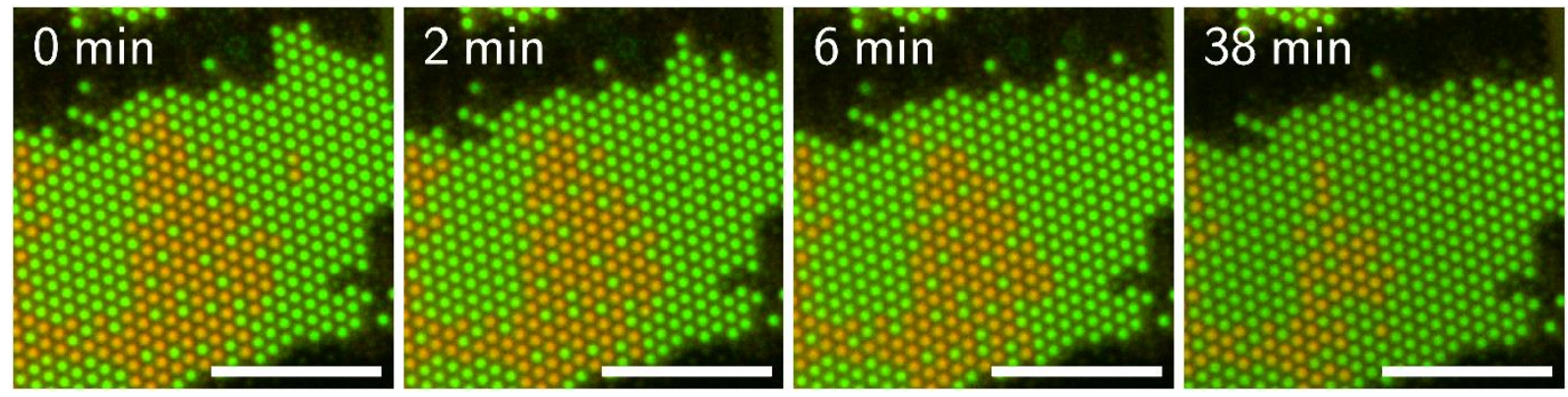

Figure 4.31: Fluorescence micrographs of a phase-separated pore-spanning membrane patch on a porous $\mathrm{Si}_{4} \mathrm{~N}_{3}$ substrate (pore diameter $0.45 \mu \mathrm{m}$ ) after spreading at $20{ }^{\circ} \mathrm{C}$, showing an apparent conversion of freestanding $l_{d}$-domains to $l_{o}$-phase. Scale bars $10 \mu \mathrm{m}$. 


\section{Results and Discussion}

The slow time scale of the lipid rearrangement can be attributed to the larger scale of the substrate and possibly differences in lipid fluidity on the pore rims. To speed up the lipid migration and reach an equilibrium state, one substrate was heated in-situ to $55^{\circ} \mathrm{C}$, well above the phase transition temperature of the lipid mixture, after spreading at $20{ }^{\circ} \mathrm{C}$. Subsequently, the substrate was cooled again to $20{ }^{\circ} \mathrm{C}$, with a rate of $0.1{ }^{\circ} \mathrm{C} / \mathrm{s}$ for both directions.
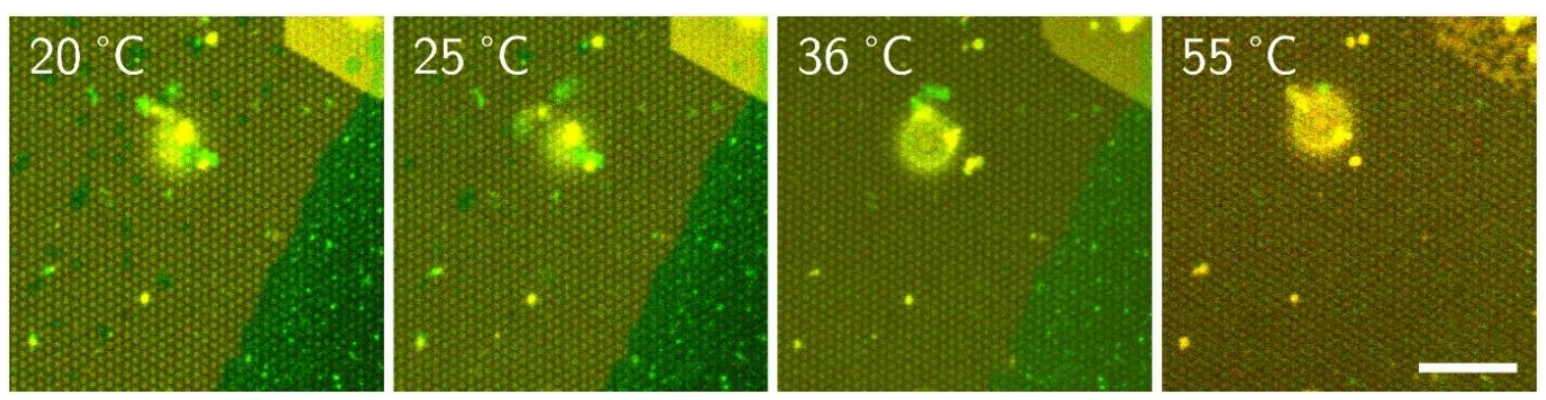

Figure 4.32: Fluorescent micrographs of a phase-separated membrane patch on a porous $\mathrm{Si}_{4} \mathrm{~N}_{3}$ substrate, spread at $20{ }^{\circ} \mathrm{C}$ and imaged during heating to $55{ }^{\circ} \mathrm{C}$ at a rate of $0.1{ }^{\circ} \mathrm{C} / \mathrm{s}$. The predominately red signal to the left of the frame is assigned as the $l_{d}$-phase region, containing several small $l_{o^{-}}$-domains.

At first, individual $l_{\sigma}$-domains in the predominately $l_{d}$-phase region were observed to disappear (see Figure $4.32,20{ }^{\circ} \mathrm{C}-36{ }^{\circ} \mathrm{C}$ ). This process was mostly complete above $30{ }^{\circ} \mathrm{C}$ and the $l_{d}$-region showed homogenous dye distribution. At $55{ }^{\circ} \mathrm{C}$ the patch showed no clear distinction anymore between the former $l_{\sigma^{-}}$and $l_{d}$-regions. Due to excessive thermal drift of the focal plane, the exact onset of lipid mixing was not observed. 

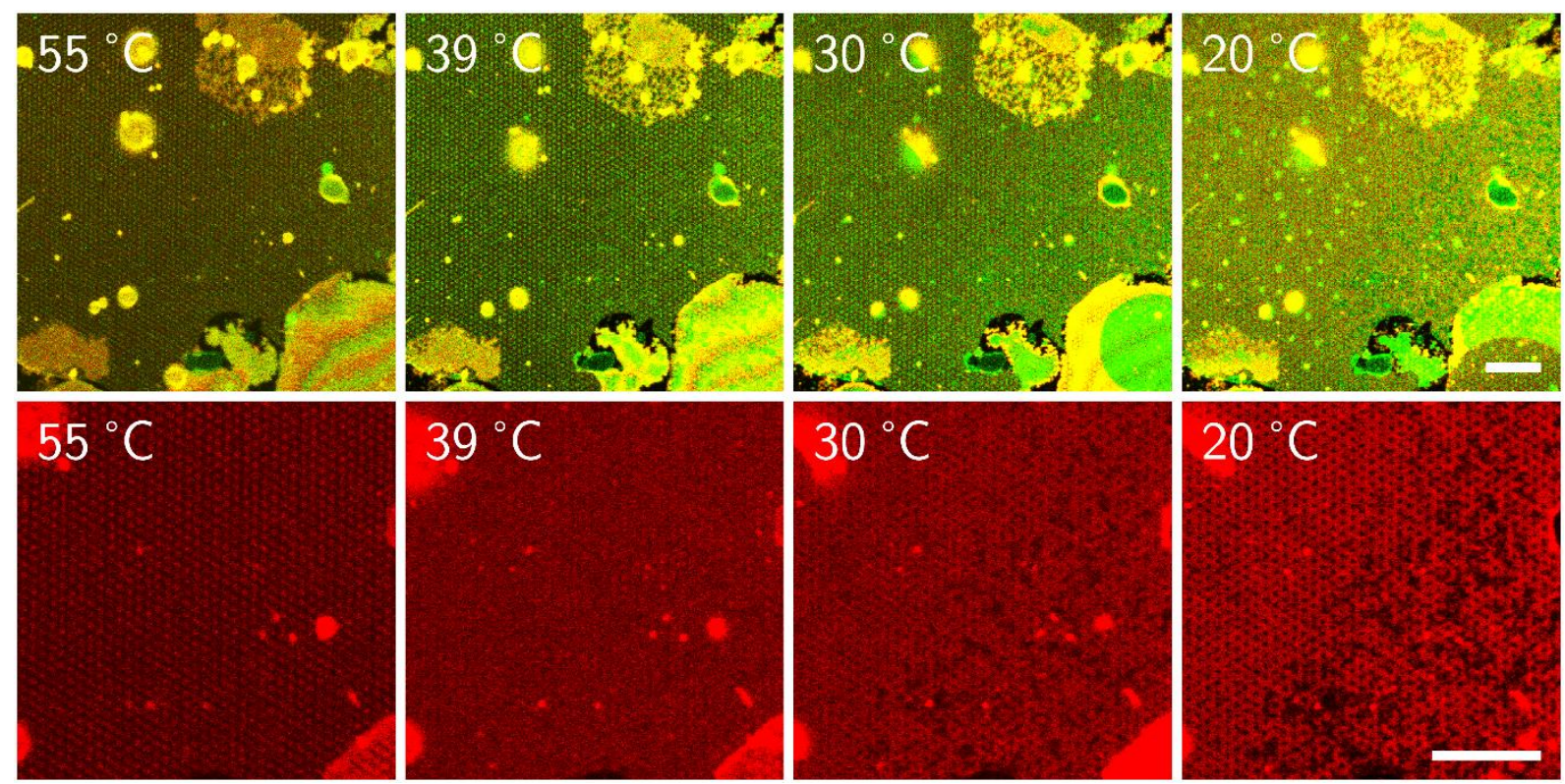

Figure 4.33 Fluorescent micrographs of the membrane patch shown in Figure 4.32 at $55{ }^{\circ} \mathrm{C}$ and successively lower temperatures. The upper row shows a wide-angle overview in both detection channels, including an adhered GUV in the lower right corner of the frame. In the lower row, a detailed view of the boundary between the $l_{d^{-}}$and $l_{o^{-}}$-region is shown. Here, only the red channel, i.e. the $l_{d}$-phase marker, is shown for clarity. Scale bars $10 \mu \mathrm{m}$.

During cooling of the substrate, the reappearance of phase separation in the planar lipid bilayer was marked by an apparent inversion of the $l_{d}$-marker distribution between freestanding and supported membrane parts (see Figure 4.33). Before the onset of phase separation, the fluorescence intensity on the pores was higher than on the rims, probably only due to quenching effects on the solid support. After re-separation of the lipid mixture, $l_{d}$-marker dye was excluded from the freestanding parts (see Figure 4.33 lower row). Simultaneously, phase separation appeared on the pore rims, but only in the former $l_{o}$-region. The process of phase separation on the planar membrane was concurrent with the reappearance of $l_{0}$-domains in adhered GUVs surrounding the membrane patch at approximately $39{ }^{\circ} \mathrm{C}$ (see Figure 4.33 upper row).

The appearance of phase separation on the pore rims suggests a higher content of sphingomyelin in the former $l_{\sigma}$-region and that lateral lipid diffusion was not complete, even in a homogeneous bilayer at elevated temperatures. However, the $l_{d}$-marker dye distributed evenly during the loss of phase separation and the lipid distribution between freestanding and supported parts can be assumed to be near equilibrium after the heating cycle. In this 


\section{Results and Discussion}

equilibrium, phase separation was observed to be governed by the underlying substrate, with $l_{\sigma^{-}}$-domains forming on the freestanding parts and $l_{d}$-phase on the solid support. This rearrangement of lipids is not limited to the case of complete lipid mixing, as evidenced by the dissolution of individual freestanding domains at temperatures below $30{ }^{\circ} \mathrm{C}$. A preference of $l_{d}$ phase for the solid supported membrane part would explain the conversion of freestanding membranes from $l_{d^{-}}$to $l_{o}$-phase observed at $20{ }^{\circ} \mathrm{C}$ (see Figure 4.31). The disappearance of isolated $l_{o}$-domains in the $l_{d}$-region with rising temperature (see Figure 4.32 ) could suggest that this preference is temperature dependent.

Taken together, the observations described in this chapter clearly show a complex rearrangement of lipids in phase separated membranes spread on a porous solid support. The appearance of a mixing region in patches on porous alumina can be attributed to lateral diffusion of lipids between the phases. The high variability observed in this process can partly

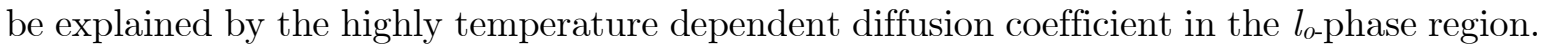
When transferred from the $\mathrm{Si}_{3} \mathrm{~N}_{4}$ substrates to functionalized AAO, the observed preference of one phase for the solid supported regions can be interpreted as the driving force of this migration. For example, a rearrangement in the $l_{d}$ region of unsaturated lipids from the freestanding parts to the pore rims would provide the concentration gradient necessary for the diffusion of these lipids into the $l_{\sigma}$-region. Variations in the magnitude of the preference, e.g. due to slight differences in substrate functionalization, would then directly influence the rate and extent of lipid diffusion.

The observed lipid mixing on AAO substrates is most likely accompanied by significant changes in lipid composition of the pore-spanning membrane parts. Moreover, these changes are not necessarily confined to the visible mixing region. They probably occur spontaneously throughout the supported bilayer, in turn causing the observed large-scale lateral diffusion.

In conclusion, the lipid redistribution on the porous alumina substrate is relatively slow but significant. In principle, permeabilization experiments on phase-separated membranes are possible in a limited time frame, but the highly variable nature of the rearrangement process precludes a consistent interpretation of permeabilization rates. Therefore, it was decided to conduct further permeabilization experiments only with non-separated lipid mixtures. 


\subsubsection{Functionalization}

The orthogonal functionalization of a porous substrate is crucial to avoid lining the pore walls with lipid material and obtain stable pore-spanning bilayers. The surface of native AAO repels lipid bilayers effectively at physiological $\mathrm{pH}$, but can be readily functionalized via silanization to enable spreading of bilayers. When temporarily masking the pore rims with a thin layer of gold by evaporation, the silane layer on the pore interior can be removed by ablation with Ar-plasma while retaining the spreading activity of the top surface. ${ }^{[93,153]}$ The AAO substrates used in this work were functionalized with this method, silanizing with (3-mercaptopropyl)triethoxysilane (MPTES) from the gas phase at $150{ }^{\circ} \mathrm{C}$ (see chapter 3.5.3.1). The procedure resulted in good bilayer adhesion, leading to vesicle rupture without significant ingress of lipid material into the pores. Frequently, however, entire substrate batches showed poor vesicle adhesion and no controlled rupture. Two factors were identified that caused reduced spreading success. Firstly, the substrate morphology influences the spreading results and reliable spreading was only found for porosities below $30 \%$ (pore diameter $<60 \mathrm{~nm})$. However, the surface activity after functionalization also varied between batches obtained from single AAO preparations with uniform morphology. Therefore, the frequent loss of bilayer adhesion was traced to unsuccessful silanization. As the envisioned incorporation of $\mathrm{Ag}$ nanowires into the substrate would greatly increase preparation complexity, unreliable silanization would potentially impact the yield of usable substrates to a significant degree. The variables governing the reaction between AAO surface and silane could not be identified and alternative orthogonal functionalization routes were investigated.

One possibility to ensure complete surface coverage with silanol groups is repeated silanization, using oxygen plasma between reactions to expose fresh reaction sites for each step. Ablation of the resulting dense layer with Ar-plasma inside the pores was deemed unreliable. However, when using (3-Aminopropyl)triethoxysilane (APTES) instead of MPTES, further reaction steps involving the amino group are possible. This route was successfully employed for the orthogonal functionalization of porous $\mathrm{Si}_{3} \mathrm{~N}_{4}$ substrates. The key step was the coating of pore walls with PEG chains via reaction of PEG-NHS with the exposed amino groups. The procedure was subsequently transferred to AAO substrates. After 


\section{Results and Discussion}

a multi-step fluid phase silanization with APTES, the silane layer was oxidized with $\mathrm{O}_{2-}$ plasma and the pore rims were covered by a thin Au layer. The pore interiors were reacted with APTES again, followed by PEGylation with PEG-NHS (see chapter 3.5.3.2). After removal of the gold mask with $\mathrm{KI} / \mathrm{I}_{2}$, no vesicle rupture was observed. The reactivation of the silanol groups on the pore rims with $\mathrm{H}_{2} \mathrm{O}$ - or $\mathrm{O}_{2}$-plasma lead to significant influx of lipid material into the pores. In the case of $\mathrm{Si}_{3} \mathrm{~N}_{4}$, mechanical removal of the gold layer with adhesive tape resulted in a clean pore rim surface, enabling bilayer adhesion. This method was not successful on AAO, however, as the gold layer adhered stronger to the substrate and could to be removed.

The contamination of the oxidized silane layer on AAO by evaporation and chemical removal of the gold mask could not be remedied without altering or removing the organic PEG layer inside the pores.

The fine balance between good coverage with silanol groups and the ability to oxidize or remove the layer selectively with $\mathrm{O}_{2}$-and Ar-plasma was found to be crucial for the orthogonal functionalization via silane chemistry. A more robust method, therefore, would have to rely on the direct activation of the pore rims without altering the native alumina inside the pores. The deposition of $\mathrm{SiO}$ via evaporation is a promising method, since studies using evaporated $\mathrm{SiO}$ on porous $\mathrm{Si}_{3} \mathrm{~N}_{4}$ substrates to obtain pore-spanning membranes were quite successful.[154]

To test the suitability of the method, porous alumina substrates were evaporated with $20 \mathrm{~nm}$ of $\mathrm{SiO}$. Samples were characterized via scanning electron microscopy and showed a continuous $\mathrm{SiO}$ layer on the pore rims, with no significant deposits inside the pores. Elevated surface roughness was observed in SEM, but could not be quantified via AFM due to the uneven morphology of the substrate on the $100 \mathrm{~nm}$ scale. Pore diameters were generally found to be equal or slightly smaller in the $\mathrm{SiO}$ layer compared to the underlying AAO. 

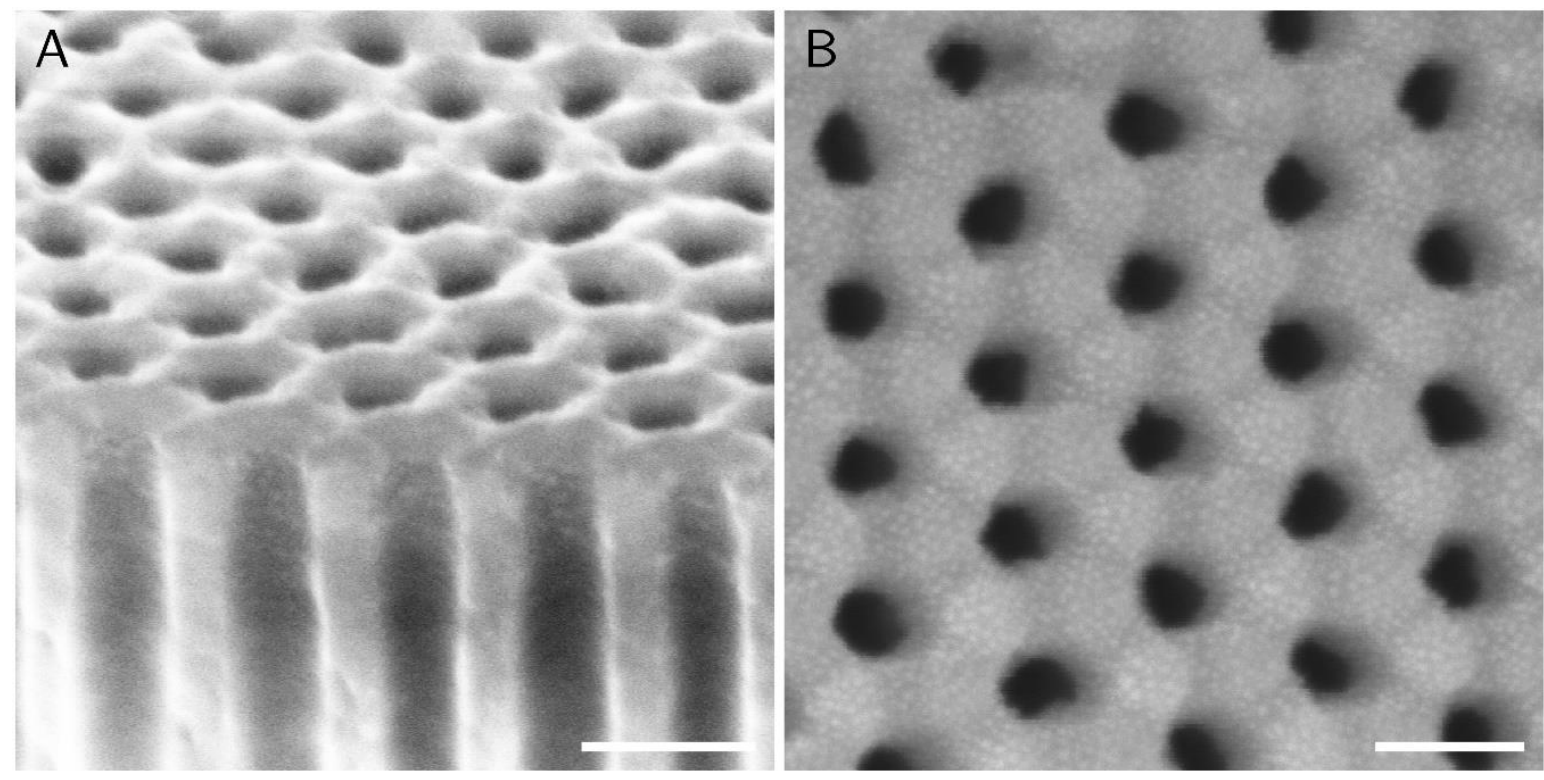

Figure 4.34: Scanning electron micrographs of $\mathrm{SiO}$ on AAO. A: side view. B: top view. Sample B was coated with $5 \mathrm{~nm}$ Au before microscopy via sputtering. Scale bars $100 \mathrm{~nm}$.

Directly after evaporation or reactivation with $\mathrm{O}_{2}$-plasma, lipid adhesion on the oxide layer was observed to be too strong, leading to uncontrolled spreading of GUVs and rupture of PSMs. This was expected, as similar results had been observed on $\mathrm{Si}_{3} \mathrm{~N}_{4}$. In that case, storing the substrate in deionized water for $1 \mathrm{~h}$ at $55{ }^{\circ} \mathrm{C}$ reduced the surface activity enough to enable controlled spreading of GUVs. ${ }^{[54]}$ A similar approach was not successful on AAO however, as the time window between PSM rupture and adhesion too weak for spreading was short and inconsistent. Vesicle adhesion and spreading was found to be more consistent in buffer containing $>0.1 \mathrm{mM} \mathrm{Ca}^{2+}$. The divalent calcium ion is well known for its ability to bridge phospholipids and promote spreading of vesicles on solid supports. ${ }^{[155]}$

A calcium-free surface treatment was found when rinsing the substrate with $1 \%$ phosphoric acid for $10 \mathrm{~s}$ before spreading. Although AAO is etched easily by phosphoric acid, no delamination of the $\mathrm{SiO}$ layer was observed. GUVs suspended in PBS buffer spread readily on the $\mathrm{SiO}$ surface after acid treatment and PSMs were found to be stable over time. Moreover, after removing lipid material by rinsing with ethanol, the surface could be reactivated by a short etching in phosphoric acid. This resulted in a clean surface, providing an opportunity to repeatedly adhere and spread GUVs on a given substrate. 

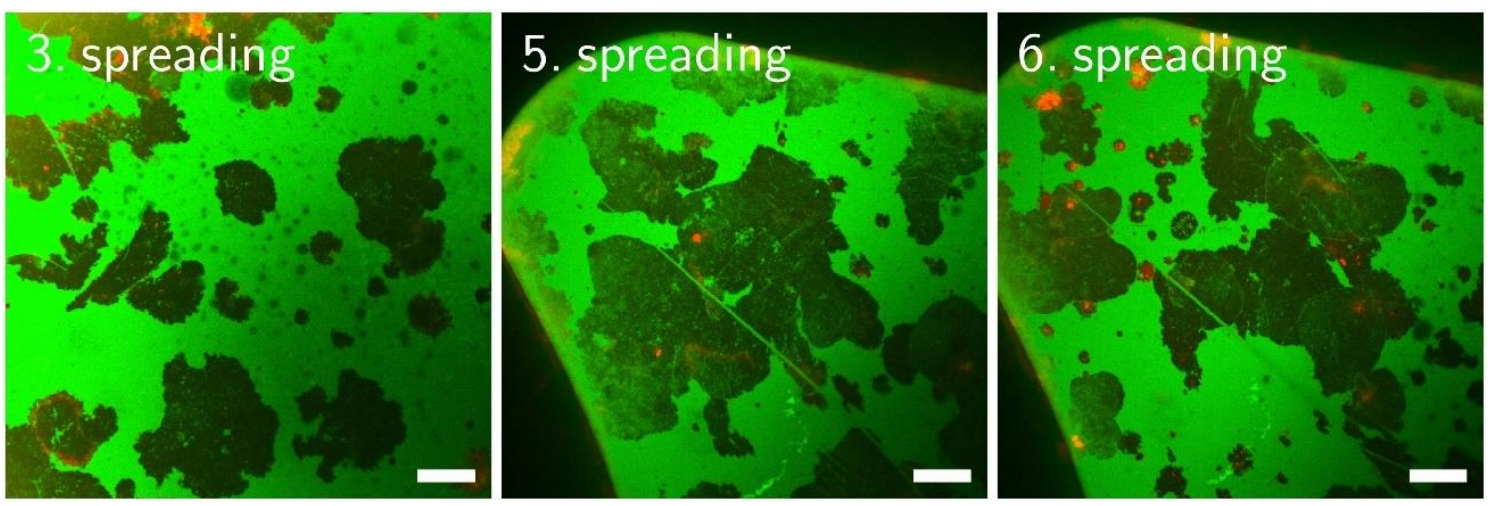

Figure 4.35: Confocal micrographs of a single AAO substrate after repeated spreading of GUVs. Between measurements, the substrate was cleaned with ethanol, rinsed with $\mathrm{H}_{3} \mathrm{PO}_{4}$, GUVs were incubated for $10 \mathrm{~min}$, and $1 \mathrm{mM}$ pyranine was added to visualize PSMs. Scale bars $50 \mu \mathrm{m}$.

The substrates were evaporated with $\mathrm{SiO}$ in bulk and stored at room temperature for up to several months. After cleaning in $\mathrm{O}_{2}$-plasma (20 mbar, $30 \mathrm{~s}$ at $60 \%$ power) and rinsing with $\mathrm{H}_{3} \mathrm{PO}_{4}$, consistent bilayer adhesion and PSM stability was found between measurements. When incubating with a large number of GUVs, spreading of vesicles at the edges of already spread membrane patches was observed. Bleaching of single patches adjacent to each other revealed diffusion of lipids between them. This fusion of GUVs to a continuous bilayer is reminiscent of the fusion and spreading of SUVs or LUVs on solid supports ${ }^{[156,157]}$ and has been described for the spreading of GUVs on glass at high vesicle densities. ${ }^{[158]}$ On silanized AAO samples fusion was never observed. In an attempt to maximize surface coverage, one sample was flushed continuously with GUV suspension after initial sedimentation. Bilayer patches were observed to rapidly grow by vesicle fusion, but the subsequent dye exclusion experiment showed PSMs only in regions covered during the initial static phase. This suggests that shear forces promote the uncontrolled rupture of vesicles at patch edges, leading to flow of lipid material onto the substrate and spreading along the pore rims. Pore-spanning patches and regions where only the rims were covered

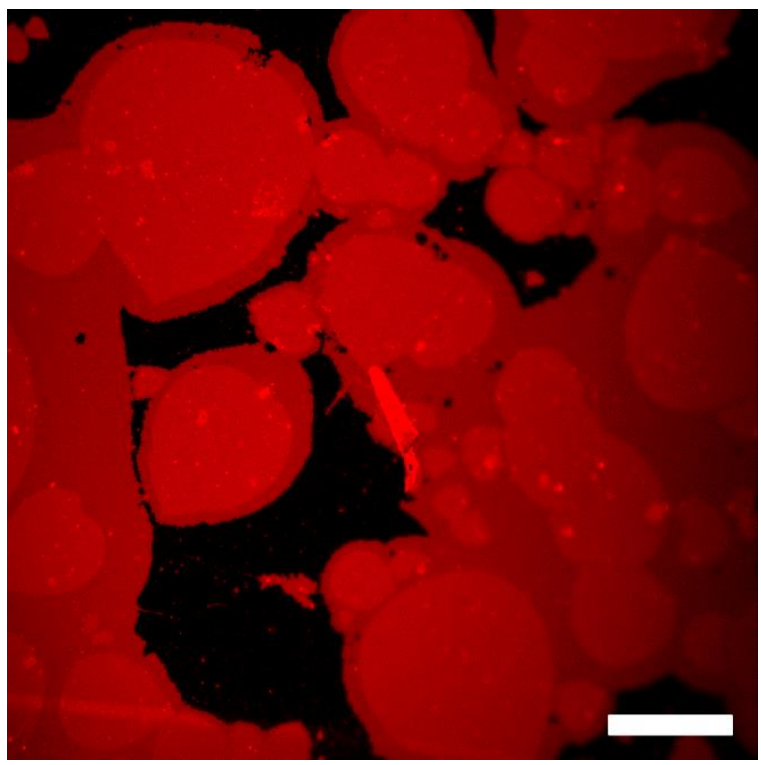

Figure 4.36 Confocal micrograph of membrane patches on $\mathrm{SiO}$-coated $\mathrm{AAO}$ after agitated spreading of GUVs. Scale bar $50 \mu \mathrm{m}$. 
could be readily distinguished by their respective membrane fluorescence intensity (see Figure 4.36).

In conclusion, the orthogonal functionalization of porous alumina via direct $\mathrm{SiO}$ deposition proofed reliable and supported stable PSMs in good yield. Consequently, it was selected as the most suitable option for the further development of the permeabilization assay.

\subsubsection{Nanoelectrode array}

The closed pores of the AAO substrate allow the quantitative observation of dye flux across the PSMs. On the downside, external access to the compartment below the bilayer is not possible and electrochemical gradients across the membrane can only be applied to some extent by exchanging the supernatant. The pore bottoms of AAO can readily be opened via etching, giving access to both aqueous compartments around the bilayer. This approach, however, has the distinct disadvantage that gradients can only be applied with complete coverage of the substrate by PSMs. Moreover, even with complete coverage, fluorescent dyes would diffuse quickly between the pores, losing all localization information in the process. By incorporating metal electrodes into the substrate pores, both compartmentalization and access to the electrochemical potential across the membrane can be combined.

As the nanowires cannot be addressed individually and complete coverage of the substrate with PSMs is unfeasible at the moment, care must be taken in the choice of electrode material. When applying a potential to the electrodes, current can freely flow through open pores and distort the actual potential across PSMs. The solution to this problem is the formation of electrodes that are non-polarizable at low currents, while developing a high overpotential when exposed to significant charge flow. With this in mind, $\mathrm{Ag} / \mathrm{AgCl}$ was chosen as the preferred electrode system.

The incorporation of silver wires into porous alumina via electrochemical deposition has been demonstrated while still attached to the aluminium substrate [159], or in the detached state. ${ }^{[160,161]}$ As only small pieces of substrate are necessary for the permeabilization assay, working with the detached AAO substrate, which can be readily split, was deemed more 


\section{Results and Discussion}

suitable. The usual approach for forming a continuous metallization of one side of the alumina is the deposition of a thin layer of silver or gold by evaporation or sputtering. [160$163]$

In keeping with this, 30-50 nm of silver were deposited via sputtering onto AAO substrates with $60 \mathrm{~nm}$ pore diameter and opened pore bottoms. This layer showed poor adhesion in the subsequent electrodeposition, however, and oxidation of the continuous Ag surface after electrodeposition impeded contacting the nanowires. The working electrode material for silver deposition was changed to gold subsequently. The thickening of the metal layer by electrodeposition of $\mathrm{Au}$ was found to form a robust contacting interface and short $\mathrm{Au}$-wires protruding into the pores. Electrodeposition of Ag onto the Au wires resulted in well-defined, dense wires of consistent height (see Figure 4.37).

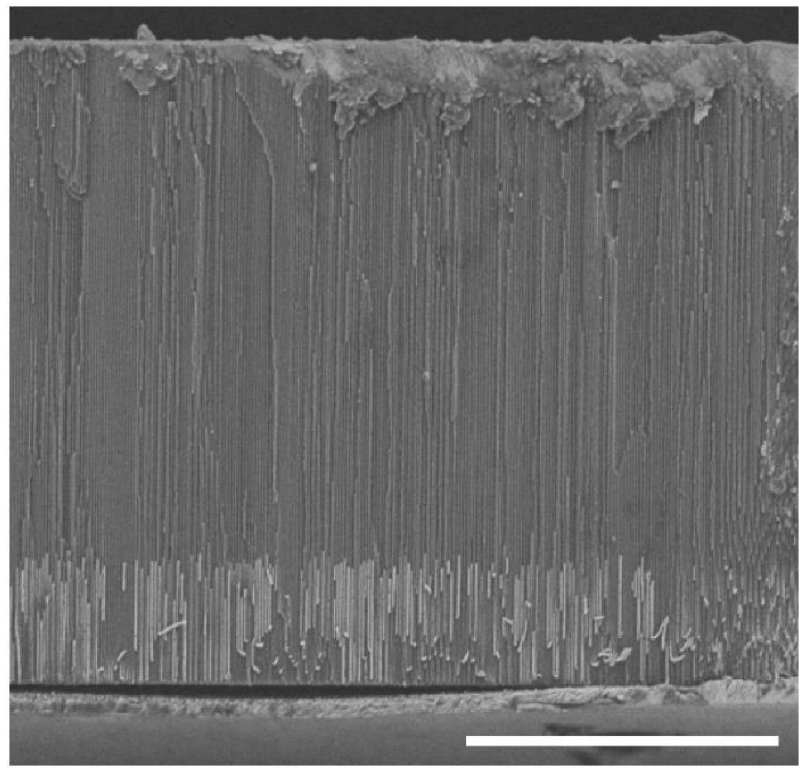

Figure 4.37: Cross-sectional SEM micrograph of silver wires embedded in AAO. Scale bar $10 \mu \mathrm{m}$.

The coulomb efficiency of the deposition, calculated from SEM micrographs of the resulting wires, was consistent but lower than reported for a comparable commercial electrolyte $(75 \%)^{[159]}$ at around $50 \%$. When factoring in this efficiency, the height of wire arrays could be controlled by monitoring deposited charge.

Success of each fabrication step was also observable by eye, as the optically clear AAO substrate noticeably shifted colors throughout the procedure. The sputtered gold layer 
resulted in a black appearance when seen through the substrate. Electrodeposition of gold produced a green or golden shine, which immediately changed to blue upon silver deposition.
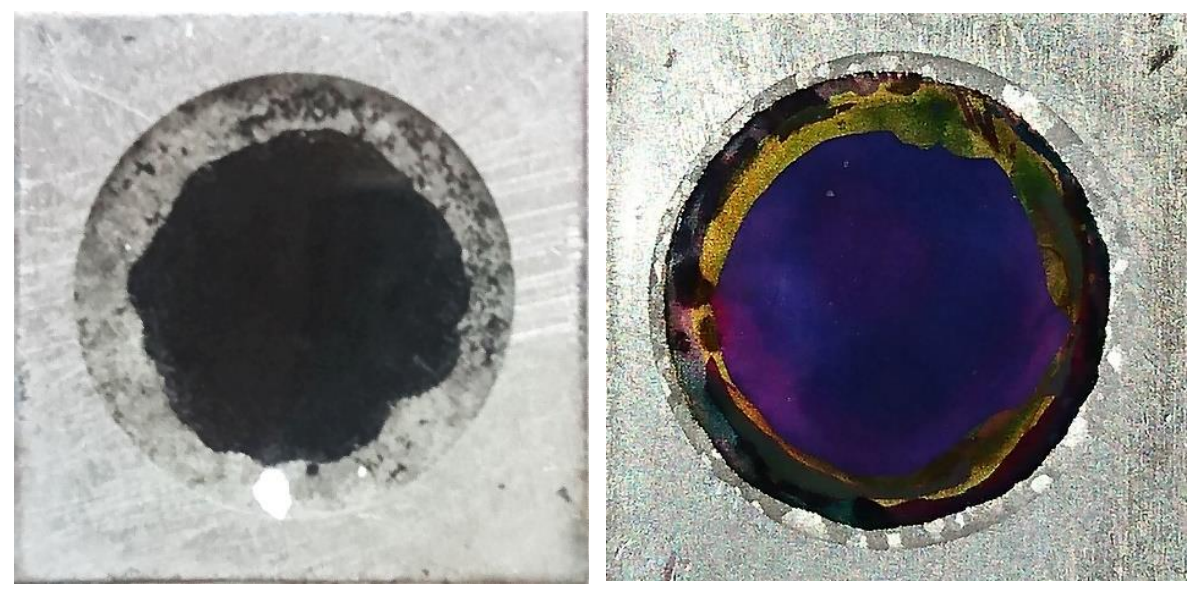

Figure 4.38: Photographs showing AAO chips during preparation of embedded nanowires. View from backside in respect to the anodization. After gold deposition, the porous substrate appears black. Electrodeposition generates a golden color (rim on right), which changes to blue during silver deposition (center region).

The electrochemical behavior of the incorporated silver nanowires was characterized via cyclic voltammetry in PBS saturated with $\mathrm{AgCl}$. A freshly chlorinated silver wire was used as a quasi-reference electrode. The open-circuit potential of the nanowire array was found to be near $0( \pm 10 \mathrm{mV})$ in reference to the silver wire.

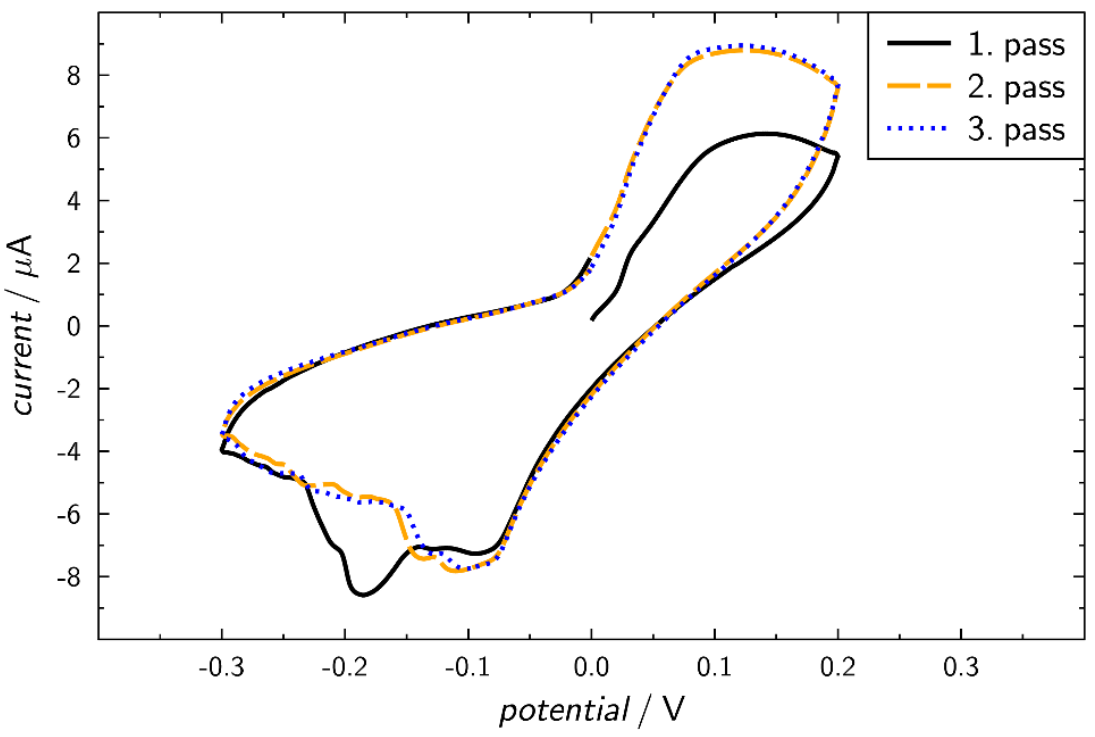

Figure 4.39: Cyclic voltammogram of the nanoelectrode array in PBS. Scanrate $100 \mathrm{mV} / \mathrm{s}$ 


\section{Results and Discussion}

As can be seen in Figure 4.39, the nanoelectrodes are readily oxidized and reduced, but both processes are hindered. At negative potentials, $\mathrm{AgCl}$ is quickly reduced and current drops as further reduction of $\mathrm{Ag}^{+}$ions is diffusion limited. At positive potentials, a drop in current is also observed. This was unexpected, as the precipitation of $\mathrm{AgCl}$ should keep the $\mathrm{Ag}^{+}$ concentration constant. Due to the relatively low chloride concentration in the buffer in comparison to typical $\mathrm{Ag} / \mathrm{AgCl}$ electrolytes $(3 \mathrm{M}), \mathrm{AgCl}$ precipitation can potentially lead to local depletion of $\mathrm{Cl}^{-}$at the silver surface. In conjunction with hindered diffusion in the pore from $\mathrm{AgCl}$ precipitate, a significant overpotential seems to be generated by this.

After extensive cycling in PBS, one sample was characterized by SEM to reveal deposits in the pores, presumably $\mathrm{AgCl}, \mathrm{Ag}$, or a mixture thereof. (see Figure 4.40). This suggests that positive currents can only be sustained for a limited time before complete silver wire oxidation. The precipitates seem to spread only towards the open pores, suggesting that the wire tips do not form a normal $\mathrm{Ag} / \mathrm{AgCl}$ junction. This supports the notion of significant overpotentials for the oxidation due to diffusion limitations at high current.
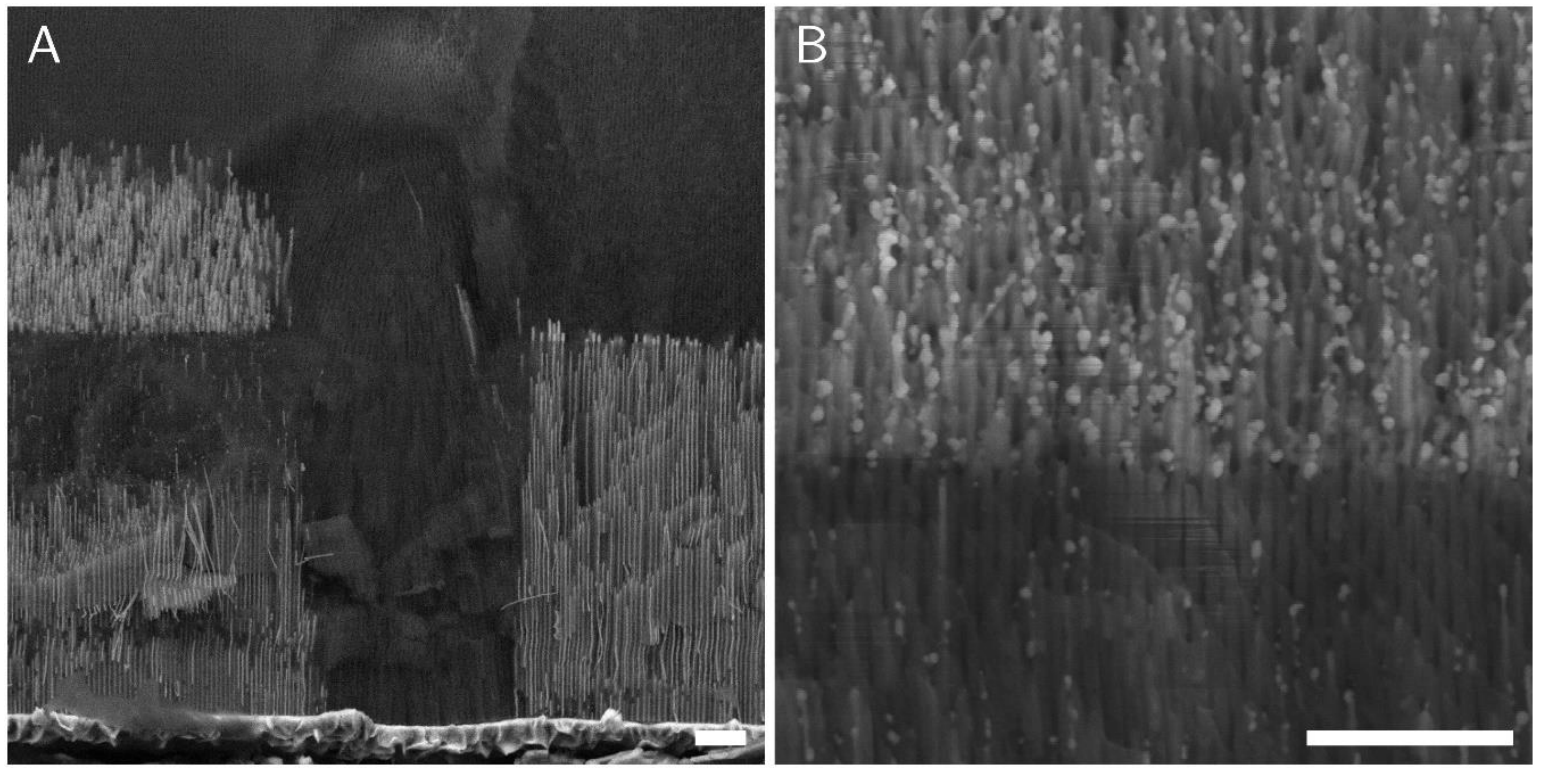

Figure 4.40: Scanning electron micrograph of nanoelectrode array after repeated oxidation and reduction in PBS buffer. A: Cross-sectional overview. The right part was covered by adhesive during cyclic voltammetry. B: Detailed view of the lower boundary of the precipitates. Scale bars $1 \mu \mathrm{m}$. 
On the whole, the exposed nanowires showed significant polarization at moderate currents, which should allow the application of a defined transmembrane potential on PSMs even at low membrane coverage of the AAO chip.

\subsubsection{Combined measurements}

After the successful preparation of nanoelectrode arrays inside the AAO, the chips were evaporated with $\mathrm{SiO}$, mounted in a custom designed conical flow cell, and electrically contacted (see chapter 3.5.5). The chip was rinsed with $1 \% \mathrm{H}_{3} \mathrm{PO}_{4}$ for $10 \mathrm{~s}$ and submersed in AgCl-saturated PBS. The electrode array was chlorinated by applying a positive potential for $60 \mathrm{~s}$ and the open-circuit potential was monitored for several minutes. Subsequently, sedimented GUV suspension (PBS/sucrose 9:1) was added and incubated for $10 \mathrm{~min}$. During spreading and subsequent rinsing with PBS, no significant change in open-circuit potential was observed, signifying a negligible influence of sucrose on the potential.

In a first proof-of-principle experiment, pyranine was added to the supernatant and dye influx into covered pores was monitored via CLSM. Then, a stepwise increasing negative potential was applied to the electrode array. PSMs were stable at $-0.1 \mathrm{~V}$ and ruptured between -0.1 and $-0.3 \mathrm{~V}$. To see if a bipolar transmembrane potential can be applied across PSMs at a sub-rupture level, membrane patches were incubated with the voltage-sensitive dye 3,3'-diethyloxacarbocyanine iodide $\left(\mathrm{DiOC}_{2}(3), 10 \mu \mathrm{M}\right.$ in $\left.\mathrm{PBS}\right)$. Subsequently, the electrode array potential was stepped to $+50 \mathrm{mV}, 0 \mathrm{mV}$, and $-50 \mathrm{mV}$ repeatedly and membrane fluorescence was monitored via CLSM. 


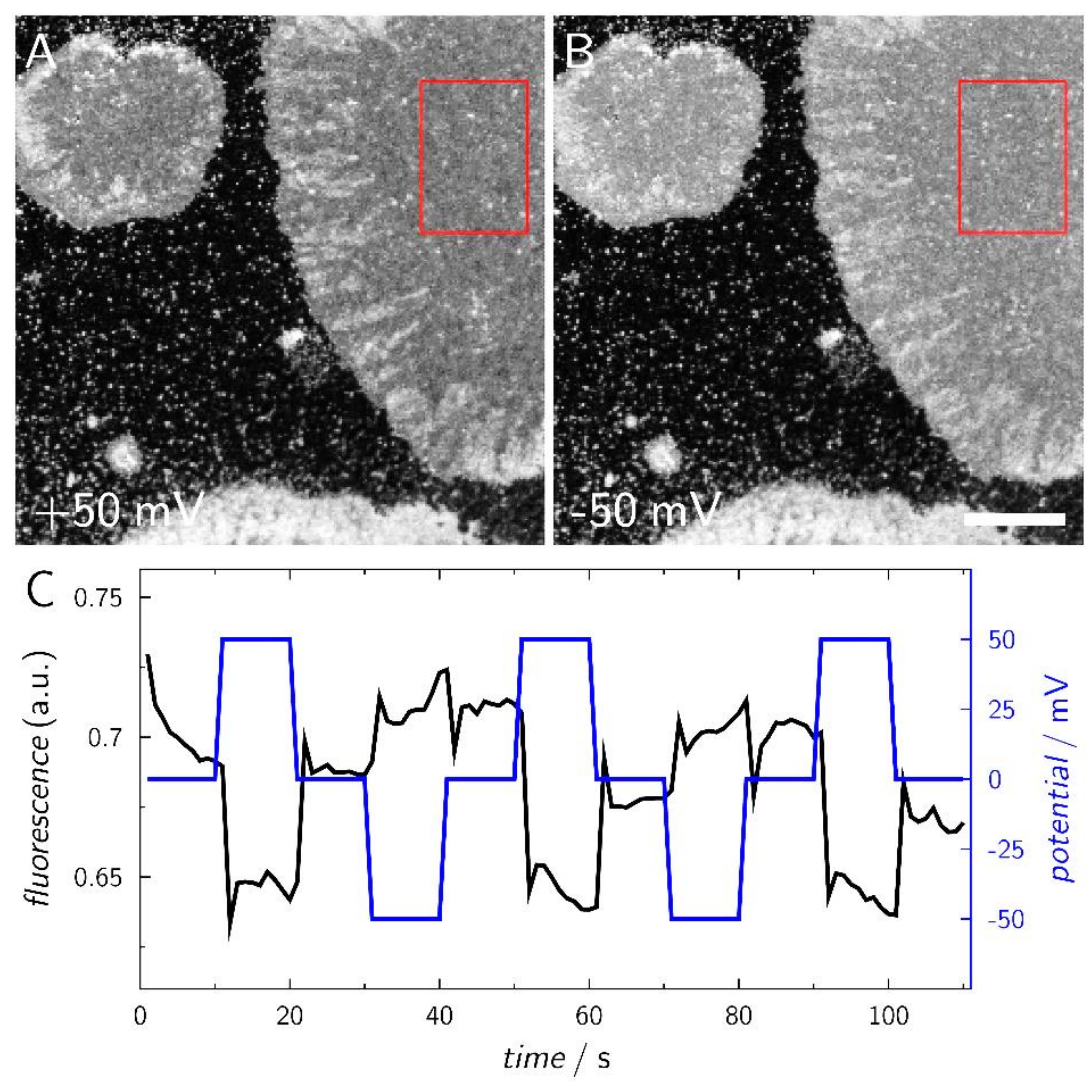

Figure 4.41: Fluorescence response of $\mathrm{DiOC}_{2}(3)$ to a transmembrane potential. A and B: Example confocal micrographs at $\lambda_{e x}=488 \mathrm{~nm}$ and $\lambda_{e m}=510-520 \mathrm{~nm}$ of a pore-spanning POPC membrane at potentials of $50 \mathrm{mV}$ and $-50 \mathrm{mV}$, respectively. Scale bar $20 \mu \mathrm{m}$. C: Time course of fluorescence intensity and applied potential.

Emission was evaluated around the maximum of around $520 \mathrm{~nm}$ in $10 \mathrm{~nm}$ intervals between $490 \mathrm{~nm}$ and $650 \mathrm{~nm}$, but changes in fluorescence intensity were uniform across the spectrum. Red fluorescing aggregates, which have been reported for bacterial cells incubated with $\mathrm{DiOC}_{2}(3)$ at negative potentials ${ }^{[164]}$ were not observed for either polarity. Although ratiometric measurements were not possible, a qualitative assessment of bilayer polarization could be extracted. As can be seen in Figure 4.41, absolute bilayer fluorescence inversely follows the applied potential. In the case of positive pulses, the change in fluorescence intensity is spontaneously reversible, while negative pulses cause an increase in fluorescence that remains constant until the polarity is actively reversed. This hysteresis is most likely related to the dye, and cannot be interpreted without further insight into the exact mechanism of the observed voltage sensitivity of $\mathrm{DiOC}_{2}(3)$ in PSMs. Nevertheless, the clear 
correlation between potential and fluorescence suggests that a reproducible transmembrane potential can be generated across PSMs by the nanoelectrode array.

\subsubsection{1 pH-change}

After the successful demonstration of an externally generated transmembrane potential, the question arose, whether proton transport across the bilayer can be induced by this potential. To investigate this possibility, a $\mathrm{pH}$-sensitive fluorophore was encapsulated inside the substrate by spreading GUVs in a $1 \mathrm{mM}$ solution of pyranine at $\mathrm{pH}$ 7.4. After rinsing with clear buffer, the pyranine trapped under membrane patches was imaged with sequential excitation at $405 \mathrm{~nm}$ and $458 \mathrm{~nm}$, allowing the ratiometric measurement of $\mathrm{pH}$ inside the pores. ${ }^{123]}$ Potentials of $\pm 25 \mathrm{mV}$ to $\pm 75 \mathrm{mV}$ were applied to the electrode array in $10 \mathrm{~s}$ intervals. A fast response to the pulses was measured in the ratio of fluorescence intensities excited at $405 \mathrm{~nm}$ and $458 \mathrm{~nm}$. A decrease in the ratio proportional to the applied potential was observed for positive voltages, indicating a rise in $\mathrm{pH}$ inside the pores.
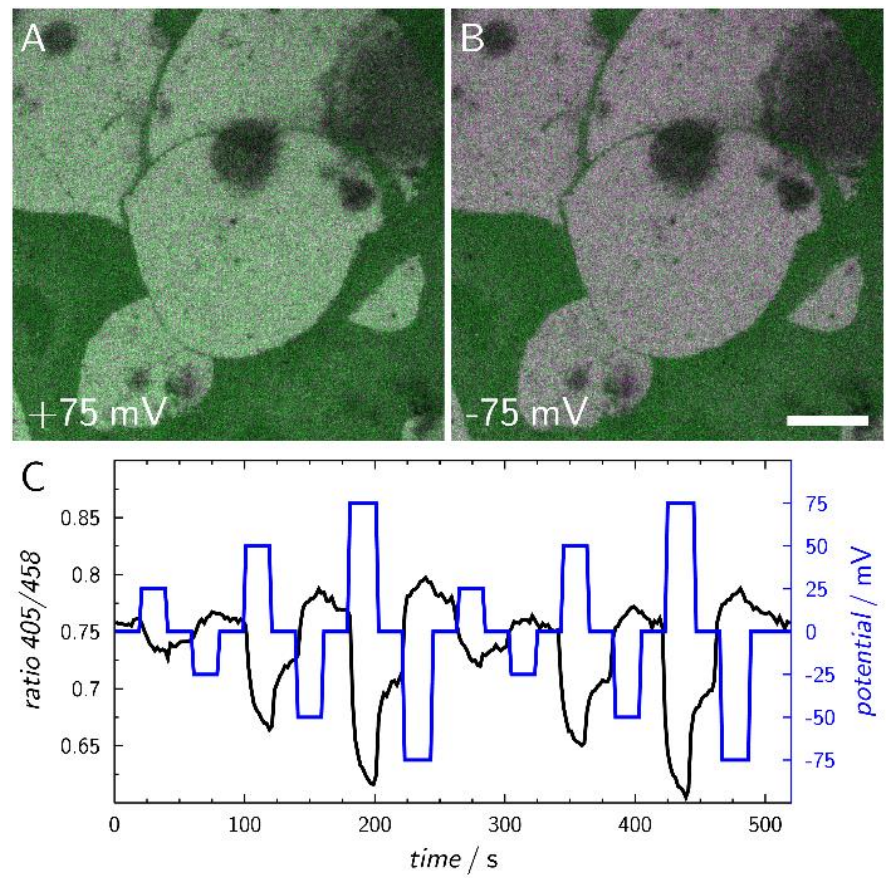

Figure 4.42: Fluorescence response of pyranine enclosed in porous AAO to a transmembrane potential. $\mathrm{A}$ and B: Example confocal micrographs at $\lambda_{e x}=405 \mathrm{~nm}$ (magenta) and $\lambda_{e x}=458 \mathrm{~nm}$ (green) of the cavities below a pore-spanning POPC membrane at potentials of $75 \mathrm{mV}$ and $-75 \mathrm{mV}$, respectively. Scale bar $20 \mu \mathrm{m}$. C: Time course of the ratio of the fluorescence intensities and applied potential. 


\section{Results and Discussion}

The polarity of the response is consistent with the notion of proton translocation along the electrochemical gradient. The inverted reaction to negative pulses was significant but less pronounced. The cause for this asymmetry in the response cannot be ascertained without further study. Higher currents were measured for the positive pulses, which could lead to a reduction of the effective potential at the AAO surface. Nonlinear dye response or the buffer capacity of the pore wall surface are also conceivable influences. The pyranine adsorbed on the non-covered pores around the membrane patches showed no ratiometric reaction to the changing electrode potential.

The changes in fluorescence showed hysteresis, but were repeatable. This suggests that proton flux across the membrane is induced by the electric field without permanent changes to the lipid bilayer. Moreover, the hysteresis of the signal in respect to the open-circuit potential indicates slow dissipation of the generated proton gradient and, therefore, defectfree PSMs.

\subsubsection{Permeabilization assay}

The established permeabilization assay was expanded to AAO substrates with embedded silver nanoelectrodes. The experiment was kept similar to the procedure described in earlier work $^{[28]}$. The three key differences were the functionalization with SiO instead of MPTES, the use of a custom designed flow cell, and finally the incorporation of nanoelectrodes into the substrate, allowing the application of a constant potential during AMP incubation. The substrate was mounted in the flow cell, electrically contacted and activated with $\mathrm{H}_{3} \mathrm{PO}_{4}$. The electrode array was freshly conditioned by anodization in the buffer and checked for anomalies by cyclovoltammetry. Pore-spanning membranes were generated by spreading of POPC GUVs and $1 \mathrm{mM}$ pyranine was added to the buffer. Influx of dye into spanned pores was then monitored via confocal microscopy. A line crossing a membrane patch was selected as ROI and continuously imaged as a high-resolution z-stack line-scan. As a benchmark experiment, $0.5 \mu \mathrm{M}$ melittin was added to the buffer, but no potential was applied. Membrane permeabilization is expected to occur in the course of minutes at this concentration. ${ }^{[62]}$ Directly after melittin addition, a steep increase of pyranine fluorescence 
beneath the pore-spanning patch was observed, indicating permeabilization. However, an unexpected linear increase in intensity followed the initial dye influx. Eventually, fluorescent intensity in the permeabilized regions exceeded that of an uncovered region, showing that an unknown factor influences pyranine fluorescence.
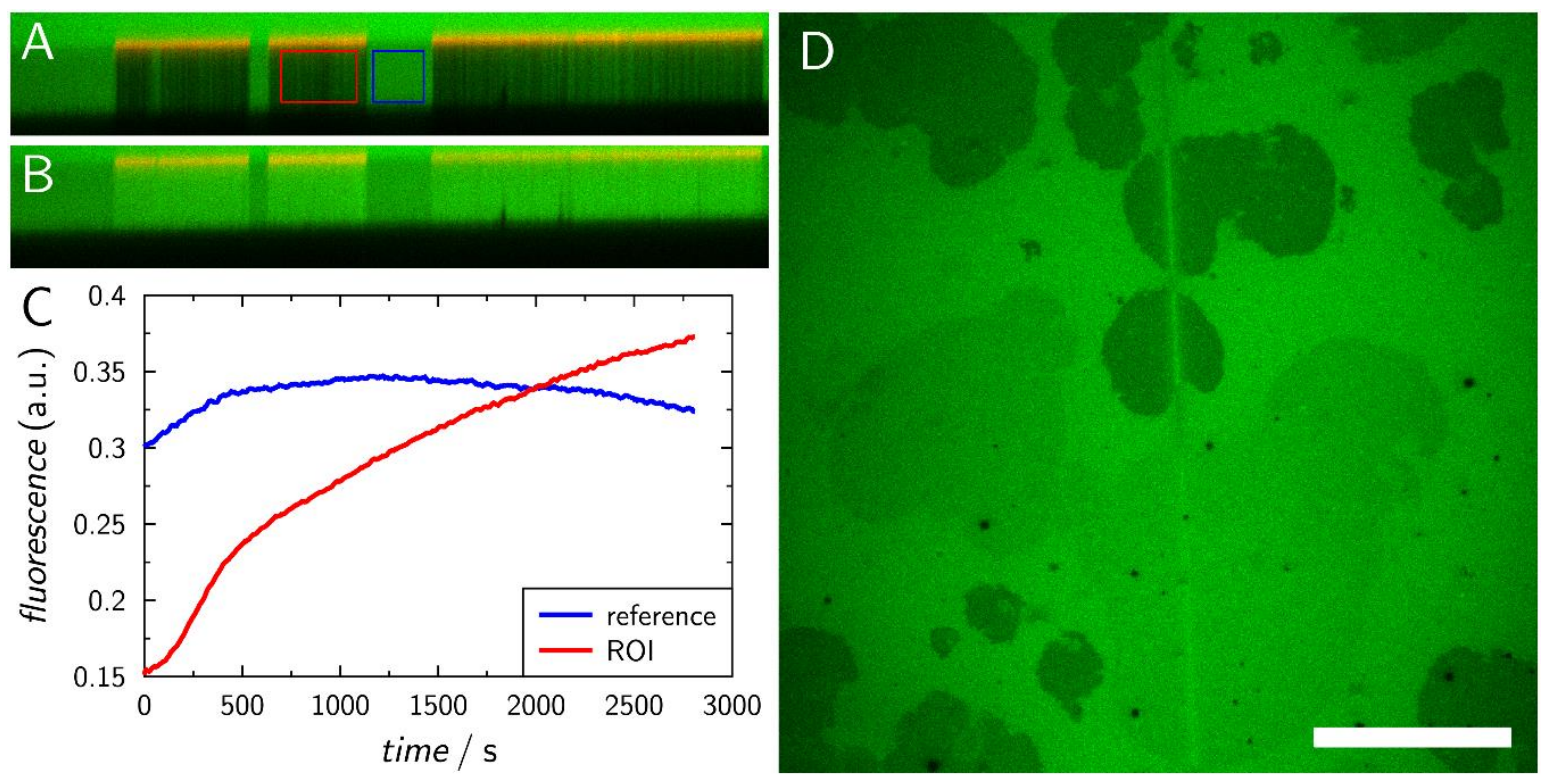

Figure 4.43: Permeabilization of PSMs by $0.5 \mu \mathrm{M}$ melittin. A and B: Confocal z-line scans across a membrane patch before and 45 min after addition of melittin, showing influx of pyranine into the substrate. C: Mean fluorescent intensities of free and spanned pore regions over time. D: Confocal micrograph of the substrate cavities after the experiment. Scale bar $50 \mu \mathrm{m}$.

After the experiment, a control micrograph of the substrate was obtained (see Figure 4.43D), which revealed two distinct abnormalities. Firstly, the apparent heterogeneity in dye influx between different patches is not expected for bilayers composed of POPC. While some variability in the rate of permeabilization is conceivable, the final intensity is expected to be nearly equal to that of uncovered regions even for lower melittin concentrations. ${ }^{[62]}$ Secondly, a clear difference between the patches and the line-scan region is visible. The scanline illuminated during the assay shows a slight reduction in intensity on the free substrate, which can be explained by bleaching of adsorbed fluorophore. In the region of patches, however, an increase in intensity can be observed. This is a clear indication of a photo-induced effect on pyranine fluorescence other than bleaching. $\mathrm{As} \mathrm{AgCl}$ is known to decompose in light of a wide range of wavelengths, ${ }^{[165]}$ the chloride deposits described in chapter 4.3 .3 are most likely 


\section{Results and Discussion}

altered in the region of high illumination. An interaction between pyranine and $\mathrm{AgCl}$ in the pores would explain the observations to some degree.

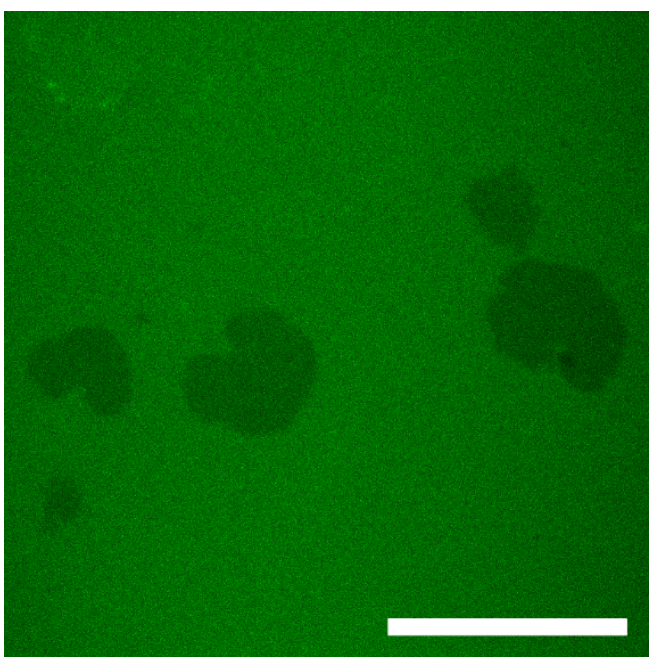

Figure 4.44: Confocal micrograph of AAO nanoelectrode substrate incubated with pyranine after lysis of PSMs. Scale bar $50 \mathrm{\mu m}$.
A second experiment was conducted by imaging the substrate every $5 \mathrm{~s}$ in $\mathrm{XY}$ direction and only in one focal plane, minimizing localized illumination. The wire array was held at $-50 \mathrm{mV}$. The addition of $10 \mu \mathrm{M}$ melittin and subsequent change of the potential down to $-800 \mathrm{mV}$ had little effect on the intensity in membrane covered regions. Even after rinsing the substrate with $1 \%$ TritonX-100, completely removing the lipid bilayer, the formerly covered regions showed decreased fluorescence when incubated with pyranine. (see Figure 4.44) 


\subsubsection{Discussion}

Phase separation

To elucidate the mechanism of this mixing, phase-separated GUVs were spread on oxidized $\mathrm{Si}_{3} \mathrm{~N}_{4}$ where a significant redistribution of lipids between the f-PSMs on the pores and the sPSMs on the rims was observed. At room temperature, a metastable system was seen, with $l_{d}$-phase f-PSMs being replaced by $l_{o}$-phase one by one. When cycling the temperature above the mixing temperature and back, a lateral diffusion of $l_{d}$-marker dye into the $l_{o}$-region was observed, followed by accumulation on the solid-supported parts upon cooling.

For the case of adhered GUVs, it has been theorized that the lipid distribution between phases is changed as soon as a so called "affinity contrast" acts on a phase-separated bilayer system. ${ }^{[166]}$ This means that if the two phases have a different affinity for the adhered region, the phase diagram is changed and the lipid composition and area of each phase is shifted. In the case of a porous substrate with two large phase domains, this new equilibrium is not immediately reached after spreading, as long-range diffusion between the regions must take place. The source of the different affinities of $l_{\sigma^{-}}$and $l_{t}$ phase can be explained by either specific interactions with the surface, ${ }^{[167]}$ or the higher bending rigidity of the $l_{\sigma}$-phase and the resulting higher energy on rough surfaces, where close adhesion to the substrate induces curvature in the membrane. ${ }^{[168,169]}$

Similar effects of lipid redistribution between freestanding and supported membranes were found on porous $\mathrm{Si}_{3} \mathrm{~N}_{4}$ substrates coated with $\mathrm{SiO}$. Here, $1_{0}$-phase was preferentially found on f-PSMs, and in the majority of preparations $l_{\sigma}$-phase was exclusively found in the porespanning f-PSMs, while $l_{d}$-phase followed the rim structure. These phase-separated PSMs were found to be a valuable tool in the investigation of the influence of membrane-bound substances on the lipid phase distribution. ${ }^{[154]}$ In the case of nanoporous substrates with structures below the resolution limit, on the other hand, no information about the specific lipid distribution between f-PSM and s-PSM can be obtained. 


\section{Results and Discussion}

This leads to the conclusion, that the permeabilization of phase-separated membranes can only be assessed under the assumption that a shift to $l_{o}$-phase on the f-PSMs is slowly progressing during the measurement, complicating the interpretation. To investigate the role of specific lipids, cholesterol for example, in the activity of AMPs, non-separated mixtures of varying composition are therefore better suited for the assay. Moreover, the activity of AMPs at phase boundaries, an interesting topic in the context of the lipid raft model, is not accessible with the assay, as phase boundaries are expected to co-locate with the support structure, rather than crossing the f-PSMs in the global boundary observable by microscopy.

Nonetheless, the spreading of phase-separated GUVs on the AAO substrate demonstrated the versatility of the assay in the choice of lipids that can be investigated.

\section{Functionalization}

The success of GUV spreading was found to be unreliable on AAO substrates functionalized via gas-phase silanization, a trait that is detrimental to the goal of a highly integrated chipbased assay. While the method has distinct advantages, like the ability to change surface characteristics by the employment of different silanes, a more robust alternative was sought for the development phase of the permeabilization assay.

With the direct orthogonal functionalization via evaporation of $\mathrm{SiO}$ onto the pore rims, this goal was achieved. Not only was it possible to generate stable PSMs on the AAO, the $\mathrm{SiO}$ coating even allowed multiple consecutive recycling steps and repeated spreading on one and the same chip. This demonstrates the feasibility of high throughput in a lab-on-a-chip style permeabilization assay based on the AAO permeabilization assay.

The main concern with $\mathrm{SiO}$ coatings is the undefined oxidation state of the surface. Excess oxygen can be introduced during evaporation, forming unspecified $\mathrm{SiO}_{\mathrm{x}=1-2}$, and the composition depends significantly on process parameters like residual pressure and deposition rate. ${ }^{[170]}$ This can lead to varying degrees of quenching of near fluorophores, ${ }^{[171]}$ as observed e.g., with fluorescently labeled lipid bilayers on $\mathrm{SiO}$ functionalized $\mathrm{Si}_{3} \mathrm{~N}_{4}$ substrates. ${ }^{[154]}$ For the permeabilization assay this means that conclusions about bilayer lysis and removal of lipid material cannot be drawn before careful analysis of the reproducibility. As the 
evaporation can be performed simultaneously for multiple chips, each providing many samples, systematic errors due to preparation can be easily analysed and corrected. On the main measure of interest in the assay, the dye influx, the functionalization has no influence.

\section{Transmembrane potential}

The main goal of the development project was to find a way to generate a transmembrane potential at the PSMs of the assay. Bacterial membranes are characterized by a large negative potential at their plasma membrane, ${ }^{[172,173]}$ a feature that is usually not modeled in permeabilization assays like carboxyfluorescein leakage. This is unfortunate, as the large potential across the cell membrane of bacteria is another pathway besides charge and lipid composition that can determine peptide specificity. The polycyclic antibacterial peptide nisin, for example, was shown to form pores in model membranes in a voltage-dependent fashion, exhibiting a threshold of around $-100 \mathrm{mV} .^{[174]}$ This influence is not necessarily confined to well defined, channel-like pores. An influence of transmembrane potential on the activity of gramicidin $\mathrm{S}$ has also been reported, which permeabilizes membranes by inducing transient defect of different sizes. ${ }^{[175]}$

The introduction of silver electrodes via electrochemical deposition was successfully demonstrated. After chlorination, a stable potential was observed in $\mathrm{AgCl}$ saturated buffers. For the permeabilization assay, the interaction between pyranine and presumably $\mathrm{AgCl}$ made quantitative measurements impossible. A quenching of fluorescence by $\mathrm{AgCl}$ has been reported for a variety of dyes. ${ }^{[176-178]}$ The adsorption and quenching of the dye in the porous $\mathrm{AgCl}$ precipitate would be a mechanism which can be reversed by $\mathrm{AgCl}$ decomposition, explaining the bright fluorescence of highly illuminated regions. Moreover, pyranine fluorescence could even be enhanced by plasmonic effects as elemental silver is formed upon $\mathrm{AgCl}$ deposition. ${ }^{[179]}$ Because $\mathrm{AgCl}$ can be sensitized by dyes, repeated exposure of pyranine filled and dye-free pores could lead to permanent silver deposits preferentially in filled pores, producing an irreversible fluorescence pattern in the substrate, similar to the process used in film-based color photography. ${ }^{[178]}$ 


\section{Results and Discussion}

Although no change of open-circuit potential was observed during the spreading of GUVs, dissolution of some $\mathrm{AgCl}$ during this step seems probable, as the GUV suspension was not completely saturated due to the content of $10 \%$ ion-free sucrose solution. This could lead to the observed differences in intensity between different membrane patches.

While the effect of $\mathrm{AgCl}$ on fluorescence was exaggerated in the proof-of-principle experiments due to extensive cycling of the potential before spreading, the active surface area of $\mathrm{AgCl}$ inside the pores has to be minimized to allow quantitative readout of fluorescence in the substrate. For this, the chloridation procedure would need to be changed to obtain a thin and dense $\mathrm{AgCl}$ layer, e.g. by changing electrolyte, current density, or by chemical chloridation. ${ }^{[180]}$ Typical procedures in literature are tailored to minimize the electrode impedance, and porous $\mathrm{AgCl}$ coatings are preferred for this. Electrode impedance is negligible compared to that of the PSMs on AAO, ${ }^{[181]}$ so a denser layer would pose no concerns. 


\section{Conclusion}

Several membrane model systems for the study of the interaction between antimicrobial compounds and the bacterial plasma membrane were presented in this thesis.

The model system of adhered GUVs offers the unique advantage of a stable, freestanding lipid bilayer with two aqueous compartments that can be resolved via confocal microscopy. With this system it was possible to elucidate the role of the lipid sidechains of the nucleoside antibiotic muraymycin in its antibacterial activity. It was concluded that the sidechain mainly facilitates the accumulation at the target membrane. For the development of antibiotics based on muraymycin, an amplification of this process by the employment of long hydrophobic sidechains could prove effective.

To study the depolarizing effect the cyclic peptide lugdunin has on bacteria, large unilamellar lipid vesicles were used as well-defined compartments mimicking the bacterial plasma membrane. It was shown that lugdunin does not permeabilize lipid bilayers for large anions but facilitates cation transport. The peptide insertion was indirectly shown to be the rate determining step of vesicle depolarization. Indications were found that lugdunin oligomerizes upon binding to form pores and that the conformational change necessary for insertion is slow. These findings need to be verified in further studies using fluorescence anisotropy to measure peptide binding, in conjunction with structural analysis of the resulting peptide aggregate by e.g. NMR, IR-, or CD-spectroscopy. Single channel conductance recordings could help reveal transient channel formation.

Pore-spanning membranes on a nanoporous, optically transparent substrate offer unique opportunities for the development of a chip-based assay for membrane permeabilization or transport processes in general. The versatility of the established assay was improved by employing a different orthogonal functionalization. The incorporation of a nanowire array allowed the generation of a transmembrane potential in this model system. It became apparent, however, that the electrodes can interfere with the fluorescent dye used for the quantitative readout of membrane permeabilization. Further research is warranted to reduce 


\section{Conclusion}

this interaction before complete integration of the substrate into a screening device is possible.

The utility of applying a direct transmembrane potential to pore-spanning membranes is not necessarily limited to a permeabilization assay, but opens up options for modeling a host of other important membrane processes. 


\section{Bibliography}

[1] W. A. Adedeji, THE TREASURE CALLED ANTIBIOTICS, Annals of Ibadan Postgraduate Medicine 2016, 14, 56-57.

[2] A. Fleming, Classics in infectious diseases: on the antibacterial action of cultures of a penicillium, with special reference to their use in the isolation of B. influenzae by Alexander Fleming, Reprinted from the British Journal of Experimental Pathology 10:226-236, 1929, Reviews of infectious diseases 1980, 2, 129-139.

[3] W. E. Gerabek, B. D. Haage, G. Keil, W. Wegner, Enzyklopädie Medizingeschichte, De Gruyter, Berlin, 2005.

[4] D. Koulenti, E. Xu, I. Yin Sum Mok, A. Song, D. E. Karageorgopoulos, A. Armaganidis, J. Lipman, S. Tsiodras, Novel Antibiotics for Multidrug-Resistant Gram-Positive Microorganisms, Microorganisms 2019, $\%$.

[5] B. Spellberg, J. H. Powers, E. P. Brass, L. G. Miller, J. E. Edwards, Trends in antimicrobial drug development: implications for the future, Clinical infectious diseases : an official publication of the Infectious Diseases Society of America 2004, 38, 1279-1286.

[6] R. I. Aminov, A brief history of the antibiotic era: lessons learned and challenges for the future, Frontiers in microbiology 2010, 1, 134.

[7] C. L. Ventola, The Antibiotic Resistance Crisis: Part 1: Causes and Threats, Pharmacy and Therapeutics 2015, 40, 277-283.

[8] U. Theuretzbacher, J. H. Toney, Nature's clarion call of antibacterial resistance: are we listening?, Current opinion in investigational drugs (London, England : 2000) 2006, 7, 158-166.

[9] Interagency Coordination Group on Antimicrobial Resistance, No time to wait: Securing the future from drug-resistant infections, 2019, Report to the SecretaryGeneral of the United Nations Washington.

[10] B. Aslam, W. Wang, M. I. Arshad, M. Khurshid, S. Muzammil, M. H. Rasool, M. A. Nisar, R. F. Alvi, M. A. Aslam, M. U. Qamar, M. K. F. Salamat, Z. Baloch, Antibiotic resistance: a rundown of a global crisis, Infection and drug resistance 2018, 11, 1645-1658.

[11] D. Romero-Calle, R. Guimarães Benevides, A. Góes-Neto, C. Billington, Bacteriophages as Alternatives to Antibiotics in Clinical Care, Antibiotics 2019, 8.

[12] N. Matamp, S. G. Bhat, Phage Endolysins as Potential Antimicrobials against Multidrug Resistant Vibrio alginolyticus and Vibrio parahaemolyticus: Current Status of Research and Challenges Ahead, Microorganisms 2019, 7.

[13] M. Domenech, J. Sempere, S. de Miguel, J. Yuste, Combination of Antibodies and Antibiotics as a Promising Strategy Against Multidrug-Resistant Pathogens of the Respiratory Tract, Frontiers in immunology 2018, 9.

[14] G. Andolina, L.-C. Bencze, K. Zerbe, M. Müller, J. Steinmann, H. Kocherla, M. Mondal, J. Sobek, K. Moehle, G. Malojčić, B. Wollscheid, J. A. Robinson, A 
Peptidomimetic Antibiotic Interacts with the Periplasmic Domain of LptD from Pseudomonas aeruginosa, ACS chemical biology 2018, 13, 666-675.

[15] M. Zasloff, Antimicrobial peptides of multicellular organisms, Nature 2002, 415, 389-395.

[16] M. Serpi, V. Ferrari, F. Pertusati, Nucleoside Derived Antibiotics to Fight Microbial Drug Resistance: New Utilities for an Established Class of Drugs?, Journal of medicinal chemistry 2016, 59, 10343-10382.

[17] R. Dziarski, D. Gupta, The peptidoglycan recognition proteins (PGRPs), Genome biology 2006, 7, 232.

[18] K. A. Brogden, Antimicrobial peptidespore formers or metabolic inhibitors in bacteria?, Nature Reviews Microbiology 2005, 3, 238-250.

[19] S. L. Davis, P. S. McKinnon, L. M. Hall, G. Delgado, W. Rose, R. F. Wilson, M. J. Rybak, Daptomycin versus vancomycin for complicated skin and skin structure infections: clinical and economic outcomes, Pharmacotherapy 2007, 27, 1611-1618.

[20] M. Zhang, J. Zhao, J. Zheng, Molecular understanding of a potential functional link between antimicrobial and amyloid peptides, Soft Matter 2014, 10, 7425-7451.

[21] K. A. Brogden, Antimicrobial peptidesPore formers or metabolic inhibitors in bacteria?, Nature Reviews Microbiology 2005, 3, 238-250.

[22] C. Song, C. Weichbrodt, E. S. Salnikov, M. Dynowski, B. O. Forsberg, B. Bechinger, C. Steinem, de Groot, Bert L., U. Zachariae, K. Zeth, Crystal structure and functional mechanism of a human antimicrobial membrane channel, Proceedings of the National Academy of Sciences 2013, 110, 4586-4591.

[23] L. T. Nguyen, E. F. Haney, H. J. Vogel, The expanding scope of antimicrobial peptide structures and their modes of action, Trends in biotechnology 2011, 29, 464-472.

[24] L. Yang, T. A. Harroun, T. M. Weiss, L. Ding, H. W. Huang, Barrel-Stave Model or Toroidal Model?A Case Study on Melittin Pores, Biophysical journal 2001, 81, $1475-1485$.

[25] Y. Shai, Z. Oren, From "carpet" mechanism to de-novo designed diastereomeric cellselective antimicrobial peptides, Peptides 2001, 22, 1629-1641.

[26] K. Matsuzaki, Membrane Permeabilization Mechanisms, Advances in experimental medicine and biology 2019, 1117, 9-16.

[27] R. Jelinek, S. Kolusheva, Membrane Interactions of Host-defense Peptides Studied in Model Systems, CPPS 2005, 6, 103-114.

[28] H. Neubacher, I. Mey, C. Carnarius, T. D. Lazzara, C. Steinem, Permeabilization Assay for Antimicrobial Peptides Based on Pore-Spanning Lipid Membranes on Nanoporous Alumina, Langmuir : the ACS journal of surfaces and colloids 2014, 30, 4767-4774.

[29] C. M. Galmarini, J. R. Mackey, C. Dumontet, Nucleoside analogues and nucleobases in cancer treatment, The Lancet Oncology 2002, 3, 415-424.

[30] E. de Clercq, Anti-HIV drugs: 25 compounds approved within 25 years after the discovery of HIV, International journal of antimicrobial agents 2009, 33, 307-320. 
[31] P. Roy-Burman in Recent Results in Cancer Research, Fortschritte der Krebsforschung Progrès dans les recherches sur le cancer, Vol. 25 (Ed.: P. RoyBurman), Springer Berlin Heidelberg, Berlin, Heidelberg, 1970.

[32] T. Shiraishi, T. Kuzuyama, Recent advances in the biosynthesis of nucleoside antibiotics, The Journal of antibiotics 2019, 72, 913-923.

[33] M. Winn, Goss, Rebecca J. M., K.-i. Kimura, Bugg, Timothy D. H., Antimicrobial nucleoside antibiotics targeting cell wall assembly: Recent advances in structurefunction studies and nucleoside biosynthesis, Nat. Prod. Rep. 2010, 27, 279-304.

[34] Timothy D. H. Bugg, Rachel V. Kerr, Mechanism of action of nucleoside antibacterial natural product antibiotics, J Antibiot 2019, 72, 865-876.

[35] B. M. Pinto, D. Barton (Eds.) Comprehensive natural products chemistry, / eds.-inchief Derek Barton ... ; Vol. 3, Elsevier, Amsterdam, 1999.

[36] V. Vinatier, C. B. Blakey, D. Braddick, B. R. G. Johnson, S. D. Evans, Bugg, Timothy D. H., In vitro biosynthesis of bacterial peptidoglycan using D-Cyscontaining precursors: fluorescent detection of transglycosylation and transpeptidation, Chemical communications (Cambridge, England) 2009, 40374039.

[37] A. Bouhss, A. E. Trunkfield, Bugg, Timothy D. H., D. Mengin-Lecreulx, The biosynthesis of peptidoglycan lipid-linked intermediates, FEMS microbiology reviews 2008, 32, 208-233.

[38] L. A. McDonald, L. R. Barbieri, G. T. Carter, E. Lenoy, J. Lotvin, P. J. Petersen, M. M. Siegel, G. Singh, R. T. Williamson, Structures of the muraymycins, novel peptidoglycan biosynthesis inhibitors, Journal of the American Chemical Society 2002, 124, 10260-10261.

[39] T. Teshima, K. Konishi, T. Shiba, Synthesis of L-Epicapreomycidine, BCSJ 1980, 53, 508-511.

[40] K. Tatsuta, N. Mikami, K. Fujimoto, S. Umezawa, H. Umezawa, The structure of chymostatin, a chymotrypsin inhibitor, The Journal of antibiotics 1973, 26, 625646.

[41] A. Yamashita, E. Norton, P. J. Petersen, B. A. Rasmussen, G. Singh, Y. Yang, T. S. Mansour, D. M. Ho, Muraymycins, novel peptidoglycan biosynthesis inhibitors: synthesis and SAR of their analogues, Bioorganic \& Medicinal Chemistry Letters 2003, 13, 3345-3350.

[42] Y.-I. Lin, Z. Li, G. D. Francisco, L. A. McDonald, R. A. Davis, G. Singh, Y. Yang, T. S. Mansour, Muraymycins, novel peptidoglycan biosynthesis inhibitors: semisynthesis and SAR of Their derivatives, Bioorganic \& Medicinal Chemistry Letters 2002, 12, 2341-2344.

[43] S. Koppermann, Z. Cui, P. D. Fischer, X. Wang, J. Ludwig, J. S. Thorson, S. G. van Lanen, C. Ducho, Insights into the Target Interaction of Naturally Occurring Muraymycin Nucleoside Antibiotics, ChemMedChem 2018. 
[44] Z. Cui, X. Wang, S. Koppermann, J. S. Thorson, C. Ducho, S. G. van Lanen, Antibacterial Muraymycins from Mutant Strains of Streptomyces sp. NRRL 30471, Journal of natural products 2018, 81, 942-948.

[45] E. G. Pamer, Resurrecting the intestinal microbiota to combat antibiotic-resistant pathogens, Science (New York, N.Y.) 2016, 352, 535-538.

[46] S. Kommineni, D. J. Bretl, V. Lam, R. Chakraborty, M. Hayward, P. Simpson, Y. Cao, P. Bousounis, C. J. Kristich, N. H. Salzman, Bacteriocin production augments niche competition by enterococci in the mammalian GI tract, Nature 2015, 526, 719-722.

[47] A. E. Reeves, M. J. Koenigsknecht, I. L. Bergin, V. B. Young, Suppression of Clostridium difficile in the gastrointestinal tracts of germfree mice inoculated with a murine isolate from the family Lachnospiraceae, Infection and immunity 2012, 80, 3786-3794.

[48] M. Sassone-Corsi, S.-P. Nuccio, H. Liu, D. Hernandez, C. T. Vu, A. A. Takahashi, R. A. Edwards, M. Raffatellu, Microcins mediate competition among Enterobacteriaceae in the inflamed gut, Nature 2016, 540, 280-283.

[49] J. W. Keith, E. G. Pamer, Enlisting commensal microbes to resist antibioticresistant pathogens, The Journal of experimental medicine 2019, 216, 10-19.

[50] A. Dobson, P. D. Cotter, R. P. Ross, C. Hill, Bacteriocin production: a probiotic trait?, Applied and environmental microbiology 2012, 78, 1-6.

[51] A. Zipperer, M. C. Konnerth, C. Laux, A. Berscheid, D. Janek, C. Weidenmaier, M. Burian, N. A. Schilling, C. Slavetinsky, M. Marschal, M. Willmann, H. Kalbacher, B. Schittek, H. Brötz-Oesterhelt, S. Grond et al., Human commensals producing a novel antibiotic impair pathogen colonization, Nature 2016, 535, 511-516.

[52] S. Böcher, B. Tønning, R. L. Skov, J. Prag, Staphylococcus lugdunensis, a common cause of skin and soft tissue infections in the community, Journal of clinical microbiology 2009, 47, 946-950.

[53] L. Bieber, G. Kahlmeter, Staphylococcus lugdunensis in several niches of the normal skin flora, Clinical microbiology and infection : the official publication of the European Society of Clinical Microbiology and Infectious Diseases 2010, 16, 385388.

[54] B. Krismer, M. Liebeke, D. Janek, M. Nega, M. Rautenberg, G. Hornig, C. Unger, C. Weidenmaier, M. Lalk, A. Peschel, Nutrient limitation governs Staphylococcus aureus metabolism and niche adaptation in the human nose, PLoS pathogens 2014, 10, e1003862.

[55] K. Bitschar, B. Sauer, J. Focken, H. Dehmer, S. Moos, M. Konnerth, N. A. Schilling, S. Grond, H. Kalbacher, F. C. Kurschus, F. Götz, B. Krismer, A. Peschel, B. Schittek, Lugdunin amplifies innate immune responses in the skin in synergy with host- and microbiota-derived factors, Nature communications 2019, 10, 2730.

[56] I. Mey, M. Stephan, E. K. Schmitt, M. M. Müller, M. Ben Amar, C. Steinem, A. Janshoff, Local membrane mechanics of pore-spanning bilayers, Journal of the American Chemical Society 2009, 131, 7031-7039. 
[57] P. Mühlenbrock, K. Herwig, L. Vuong, I. Mey, C. Steinem, Fusion Pore Formation Observed During SNARE-Mediated Vesicle Fusion with Pore-Spanning Membranes, 2020 .

[58] S. Spindler, J. Sibold, R. Gholami Mahmoodabadi, C. Steinem, V. Sandoghdar, High-Speed Microscopy of Diffusion in Pore-Spanning Lipid Membranes, Nano letters 2018, 18, 5262-5271.

[59] M. Stephan, I. Mey, C. Steinem, A. Janshoff, Combining reflectometry and fluorescence microscopyAn assay for the investigation of leakage processes across lipid membranes, Analytical chemistry 2014, 86, 1366-1371.

[60] Lau, King Hang Aaron, L.-S. Tan, K. Tamada, M. S. Sander, W. Knoll, Highly Sensitive Detection of Processes Occurring Inside Nanoporous Anodic Alumina Templates: A Waveguide Optical Study, J. Phys. Chem. B 2004, 108, 1081210818.

[61] T. D. Lazzara, I. Mey, C. Steinem, A. Janshoff, Benefits and limitations of porous substrates as biosensors for protein adsorption, Analytical chemistry 2011, 83, $5624-5630$.

[62] Henrik Neubacher, Dissertation, Georg August Universität Göttingen, Göttingen, 2017.

[63] T. Kaufeld, C. Steinem, C. F. Schmidt, Microporous device for local electric recordings on model lipid bilayers, Nanotechnology 2015, 48, 25401.

[64] S. F. Fenz, K. Sengupta, Giant vesicles as cell models, Integr. Biol. 2012, 4, 982995.

[65] J. C. Freedman. Cell physiology sourcebook in Cell physiology sourcebook. A molecular approach (Ed.: N. Sperelakis), Acad. Press, San Diego [u.a.], 2001.

[66] B. Pozo Navas, K. Lohner, G. Deutsch, E. Sevcsik, K. A. Riske, R. Dimova, P. Garidel, G. Pabst, Composition dependence of vesicle morphology and mixing properties in a bacterial model membrane system, Biochimica et Biophysica Acta (BBA)-Biomembranes 2005, 1716, 40-48.

[67] G. Bryant, J. Pope, J. Wolfe, Low hydration phase properties of phospholipid mixtures, Eur Biophys J 1992, 21.

[68] M. I. Angelova, D. S. Dimitrov, Liposome electroformation, Faraday Discuss. Chem. Soc. 1986, 81, 303.

[69] P. Méléard, L. A. Bagatolli, T. Pott in Methods in Enzymology (Ed.: N. Düzgünes), Elsevier Academic Press, Amsterdam, London, 2009.

[70] S. L. Veatch, S. L. Keller, Miscibility phase diagrams of giant vesicles containing sphingomyelin, Physical Review Letters 2005, 94, 148101.

[71] C. Berney, G. Danuser, FRET or No FRET: A Quantitative Comparison, Biophysical journal 2003, 84, 3992-4010.

[72] O. Ries, C. Carnarius, C. Steinem, C. Ducho, Membrane-interacting properties of the functionalised fatty acid moiety of muraymycin antibiotics, Med. Chem.

Commun. 2015, 6, 879-886. 
6 Bibliography

[73] N. M. GREEN, AVIDIN. 3. THE NATURE OF THE BIOTIN-BINDING SITE, The Biochemical journal 1963, 89, 599-609.

[74] J. Piehler, A. Brecht, R. Valiokas, B. Liedberg, G. Gauglitz, A high-density poly(ethylene glycol) polymer brush for immobilization on glass-type surfaces, Biosensors and Bioelectronics 2000, 15, 473-481.

[75] K. Carvalho, L. Ramos, C. Roy, C. Picart, Giant unilamellar vesicles containing phosphatidylinositol $(4,5)$ bisphosphateCharacterization and functionality, Biophysical journal 2008, 95, 4348-4360.

[76] R. F. Chen, J. R. Knutson, Mechanism of fluorescence concentration quenching of carboxyfluorescein in liposomesEnergy transfer to nonfluorescent dimers, Analytical biochemistry 1988, 172, 61-77.

[77] J. N. Weinstein, R. Blumenthal, R. D. Klausner in Methods in Enzymology (Eds.: J. P. Segrest, J. J. Albers, W. A. Bradley, S. H. Gianturco), Elsevier, 1996.

[78] J. P. Dufour, A. Goffeau, T. Y. Tsong, Active proton uptake in lipid vesicles reconstituted with the purified yeast plasma membrane ATPase. Fluorescence quenching of 9-amino-6-chloro-2-methoxyacridine, Journal of Biological Chemistry 1982, 257, 9365-9371.

[79] T. Hotz, O. M. Schütte, H. Sieling, T. Polupanow, U. Diederichsen, C. Steinem, A. Munk, Idealizing ion channel recordings by a jump segmentation multiresolution filter, IEEE transactions on nanobioscience 2013, 12, 376-386.

[80] O. P. Hamill, A. Marty, E. Neher, B. Sakmann, F. J. Sigworth, Improved patchclamp techniques for high-resolution current recording from cells and cell-free membrane patches, Pflugers Archiv : European journal of physiology 1981, 391, 85100.

[81] H. Garty, B. Rudy, S. J. Karlish, A simple and sensitive procedure for measuring isotope fluxes through ion-specific channels in heterogenous populations of membrane vesicles, Journal of Biological Chemistry 1983, 258, 13094-13099.

[82] A. V. Koulov, J. M. Mahoney, B. D. Smith, Facilitated transport of sodium or potassium chloride across vesicle membranes using a ditopic salt-binding macrobicycle, Organic \& biomolecular chemistry 2003, 1, 27-29.

[83] H. Rottenberg, R. Moreno-Sanchez, The proton pumping activity of $\mathrm{H}+-$ ATPasesAn improved fluorescence assay, Biochimica et Biophysica Acta (BBA) Bioenergetics 1993, 1183, 161-170.

[84] S.-Y. Lee, J. A. Letts, R. MacKinnon, Functional Reconstitution of Purified Human Hv1 H+ Channels, Journal of Molecular Biology 2009, 387, 1055-1060.

[85] K. T. Howitz, R. E. McCarty, Measurement of proton-linked transport activities in pyranine loaded chloroplast inner envelope vesicles, Plant physiology 1988, 86, 9991001.

[86] G. E. Tiller, T. J. Mueller, M. E. Dockter, W. G. Struve, Hydrogenation of triton X-100 eliminates its fluorescence and ultraviolet light absorption while preserving its detergent properties, Analytical biochemistry 1984, 141, 262-266. 
[87] A. P. Li, F. Muller, A. Birner, K. Nielsch, U. Gosele, Hexagonal pore arrays with a 50-420 $\mathrm{nm}$ interpore distance formed by self-organization in anodic alumina, Journal of Applied Physics 1998, 84, 6023-6026.

[88] K. Nielsch, J. Choi, K. Schwirn, R. B. Wehrspohn, U. Gösele, Self-ordering regimes of porous aluminathe 10 porosity rule, Nano letters 2002, 2, 677-680.

[89] G. D. Sulka. Wiley-VCH Verlag GmbH \& Co. KGaA, Weinheim, Germany in Wiley-VCH Verlag GmbH \& Co. KGaA, Weinheim, Germany, 2008.

[90] C. E. Korman, M. Megens, C. M. Ajo-Franklin, D. A. Horsley, Nanopore-Spanning Lipid Bilayers on Silicon Nitride Membranes That Seal and Selectively Transport Ions, Langmuir : the ACS journal of surfaces and colloids 2013, 29, 4421-4425.

[91] B. Venkatesan, J. Polans, J. Comer, S. Sridhar, D. Wendell, A. Aksimentiev, R. Bashir, Lipid bilayer coated Al2O3 nanopore sensors: towards a hybrid biological solid-state nanopore, Biomed Microdevices 2011, 13, 671-682.

[92] J. A. Jackman, S. R. Tabaei, Z. Zhao, S. Yorulmaz, N.-J. Cho, Self-assembly formation of lipid bilayer coatings on bare aluminum oxide: overcoming the force of interfacial water, ACS applied materials $\&$ interfaces 2015, 7, 959-968.

[93] T. D. Lazzara, C. Carnarius, M. Kocun, A. Janshoff, C. Steinem, Separating attoliter-sized compartments using fluid pore-spanning lipid bilayers, ACS Nano 2011, 5, 6935-6944.

[94] E. T. Castellana, P. S. Cremer, Solid supported lipid bilayersFrom biophysical studies to sensor design, Surface Science Reports 2006, 61, 429-444.

[95] P. M. Wolny, J. P. Spatz, R. P. Richter, On the adsorption behavior of biotinbinding proteins on gold and silica, Langmuir 2010, 26, 1029-1034.

[96] M. Schwamborn, J. Schumacher, J. Sibold, N. K. Teiwes, C. Steinem, Monitoring ATPase induced $\mathrm{pH}$ changes in single proteoliposomes with the lipid-coupled fluorophore Oregon Green 488, The Analyst 2017, 142, 2670-2677.

[97] T. Förster, Zwischenmolekulare Energiewanderung und Fluoreszenz, Ann. Phys. 1948, 437, 55-75.

[98] H. Akaike in Springer Series in Statistics, Perspectives in Statistics, 0172-7397 (Eds.: E. Parzen, K. Tanabe, G. Kitagawa), Springer New York, New York, NY, 1998 .

[99] Y. L. Jeyachandran, J. A. Mielczarski, E. Mielczarski, B. Rai, Efficiency of blocking of non-specific interaction of different proteins by BSA adsorbed on hydrophobic and hydrophilic surfaces, Journal of Colloid and Interface Science 2010, 341, 136142.

[100] E. I. Silva-López, L. E. Edens, A. O. Barden, D. J. Keller, J. A. Brozik, Conditions for liposome adsorption and bilayer formation on BSA passivated solid supports, Chemistry and physics of lipids 2014, 183, 91-99.

[101] A. Müller, M. Wenzel, H. Strahl, F. Grein, T. N. V. Saaki, B. Kohl, T. Siersma, J. E. Bandow, H.-G. Sahl, T. Schneider, L. W. Hamoen, Daptomycin inhibits cell envelope synthesis by interfering with fluid membrane microdomains, Proceedings 
of the National Academy of Sciences of the United States of America 2016, 113, E7077-E7086.

[102] T. Sych, T. Schubert, R. Vauchelles, J. Madl, R. Omidvar, R. Thuenauer, L. Richert, Y. Mély, W. Römer, GUV-APMultifunctional FIJI-based tool for quantitative image analysis of Giant Unilamellar Vesicles, Bioinformatics (Oxford, England) 2018.

[103] F. A. Thomas, I. Visco, Z. Petrášek, F. Heinemann, P. Schwille, Introducing a fluorescence-based standard to quantify protein partitioning into membranes, Biochimica et biophysica acta 2015, 1848, 2932-2941.

[104] S. Aimon, J. Manzi, D. Schmidt, J. A. Poveda Larrosa, P. Bassereau, G. E. S. Toombes, Functional reconstitution of a voltage-gated potassium channel in giant unilamellar vesicles, PloS one 2011, 6, e25529.

[105] F. Duša, W. Chen, J. Witos, A. H. Rantamäki, A. W. T. King, E. Sklavounos, M. Roth, S. K. Wiedmer, Immobilization of natural lipid biomembranes and their interactions with choline carboxylates. A nanoplasmonic sensing study, Biochimica et biophysica acta. Biomembranes 2020, 1862, 183115.

[106] S. Munusamy, R. Conde, B. Bertrand, C. Munoz-Garay, Biophysical approaches for exploring lipopeptide-lipid interactions, Biochimie 2020, 170, 173-202.

[107] R. M. Peitzsch, S. McLaughlin, Binding of acylated peptides and fatty acids to phospholipid vesicles: pertinence to myristoylated proteins, Biochemistry 1993, 32, 10436-10443.

[108] C. A. Buser, C. T. Sigal, M. D. Resh, S. McLaughlin, Membrane binding of myristylated peptides corresponding to the NH2 terminus of Src, Biochemistry 1994, 33, 13093-13101.

[109] R. J. T. Houk, S. L. Tobey, E. V. Anslyn in Topics in Current Chemistry, Vol. 255 (Eds.: E. V. Anslyn, I. Stibor), Springer, Berlin, 2005.

[110] L. Li, I. Vorobyov, T. W. Allen, The different interactions of lysine and arginine side chains with lipid membranes, The journal of physical chemistry. B 2013, 117, 11906-11920.

[111] S. Leekumjorn, A. K. Sum, Molecular characterization of gel and liquid-crystalline structures of fully hydrated POPC and POPE bilayers, J. Phys. Chem. B 2007, 111, 6026-6033.

[112] K. J. Seu, L. R. Cambrea, R. M. Everly, J. S. Hovis, Influence of lipid chemistry on membrane fluidity: tail and headgroup interactions, Biophysical journal 2006, 91, $3727-3735$.

[113] Insoluble drug delivery strategiesReview of recent advances and business prospects, Acta Pharmaceutica Sinica B 2015, 5, 442-453.

[114] N. A. Schilling, A. Berscheid, J. Schumacher, J. S. Saur, M. C. Konnerth, S. N. Wirtz, J. M. Beltrán Beleña, A. Zipperer, B. Krismer, A. Peschel, H. Kalbacher, H. Brötz-Oesterhelt, C. Steinem, S. C. Grond, Synthetic Lugdunin Analogues Reveal Essential Structural Motifs for Antimicrobial Action and Proton Translocation Capability, Angewandte Chemie (International ed. in English) 2019. 
[115] R. F. Chen, J. R. Knutson, Mechanism of fluorescence concentration quenching of carboxyfluorescein in liposomesEnergy transfer to nonfluorescent dimers, Analytical biochemistry 1988, 172, 61-77.

[116] A. S. Ladokhin, W. C. Wimley, K. Hristova, S. H. White in Methods in Enzymology, Vol. 278 (Ed.: L. Brand), Acad. Press, San Diego, Calif., 1997.

[117] T. Katsu, H. Kobayashi, T. Hirota, Y. Fujita, K. Sato, U. Nagai, Structure-activity relationship of gramicidin S analogues on membrane permeability, Biochimica et Biophysica Acta (BBA) - Biomembranes 1987, 899, 159-170.

[118] E. J. Prenner, R. N.A.H. Lewis, R. N. McElhaney, The interaction of the antimicrobial peptide gramicidin $\mathrm{S}$ with lipid bilayer model and biological membranes, Biochimica et Biophysica Acta (BBA) - Biomembranes 1999, 1462, 201-221.

[119] J. Swierstra, V. Kapoerchan, A. Knijnenburg, A. van Belkum, M. Overhand, Structure, toxicity and antibiotic activity of gramicidin S and derivatives, European journal of clinical microbiology $\&$ infectious diseases : official publication of the European Society of Clinical Microbiology 2016, 35, 763-769.

[120] J. Zhang, M. Myers, M. Forgac, Characterization of the V0 domain of the coated vesicle (H+)-ATPase, Journal of Biological Chemistry 1992, 267, 9773-9778.

[121] R. Benz, G. Stark, K. Janko, P. Läuger, Valinomycin-mediated ion transport through neutral lipid membranesInfluence of hydrocarbon chain length and temperature, J Membrane Biol 1973, 14, 339-364.

[122] R. Casadio, Measurements of transmembrane $\mathrm{pH}$ differences of low extents in bacterial chromatophores, Eur Biophys J 1991, 19.

[123] K. Kano, J. H. Fendler, Pyranine as a sensitive pH probe for liposome interiors and surfaces. pH gradients across phospholipid vesicles, Biochimica et Biophysica Acta (BBA) - Biomembranes 1978, 509, 289-299.

[124] N. R. Clement, J. M. Gould, Pyranine (8-hydroxy-1,3,6-pyrenetrisulfonate) as a probe of internal aqueous hydrogen ion concentration in phospholipid vesicles, Biochemistry 1981, 20, 1534-1538.

[125] N. R. Clement, J. M. Gould, Kinetics for the development of gramicidin-induced ion permeability in unilamellar phospholipid vesicles, Biochemistry 1981, 20, 15441548.

[126] W. A. Lea, A. Simeonov, Fluorescence polarization assays in small molecule screening, Expert opinion on drug discovery 2011, 6, 17-32.

[127] C. A. Kraft, J. L. Garrido, L. Leiva-Vega, G. Romero, Quantitative analysis of protein-lipid interactions using tryptophan fluorescence, Science signaling 2009, 2, pl4.

[128] K. Matsuzaki, Control of cell selectivity of antimicrobial peptides, Biochimica et biophysica acta 2009, 1788, 1687-1692.

[129] P. Maturana, M. Martinez, M. E. Noguera, N. C. Santos, E. A. Disalvo, L. Semorile, P. C. Maffia, A. Hollmann, Lipid selectivity in novel antimicrobial 
peptides: Implication on antimicrobial and hemolytic activity, Colloids and surfaces. B, Biointerfaces 2017, 153, 152-159.

[130] T. Zhang, J. K. Muraih, B. MacCormick, J. Silverman, M. Palmer, Daptomycin forms cation- and size-selective pores in model membranes, Biochimica et biophysica acta 2014, 1838, 2425-2430.

[131] T. Podleski, J. P. Changeux, Effects associated with permeability changes caused by gramicidin A in electroplax membrane, Nature 1969, 221, 541-545.

[132] J. A. Doebler, Gramicidin toxicity in NG108-15 cells: protective effects of acetamidine and guanidine, Cell biology and toxicology 1999, 15, 279-289.

[133] J. Leung, R. S. Eisenberg, The effects of the antibiotics gramicidin A, amphotericin $\mathrm{B}$, and nystatin on the electrical properties of frog skeletal muscle, Biochimica et biophysica acta 1973, 298, 718-723.

[134] A. I. Sorochkina, E. Y. Plotnikov, T. I. Rokitskaya, S. I. Kovalchuk, E. A. Kotova, S. V. Sychev, D. B. Zorov, Y. N. Antonenko, N-terminally glutamate-substituted analogue of gramicidin A as protonophore and selective mitochondrial uncoupler, PloS one 2012, 7, e41919.

[135] T. D. Clark, L. K. Buehler, M. R. Ghadiri, Self-Assembling Cyclic $\beta 3$-Peptide Nanotubes as Artificial Transmembrane Ion Channels, Journal of the American Chemical Society 1998, 120, 651-656.

[136] M. R. Ghadiri, K. Kobayashi, J. R. Granja, R. K. Chadha, D. E. McRee, The Structural and Thermodynamic Basis for the Formation of Self-Assembled Peptide Nanotubes, Angew. Chem. Int. Ed. Engl. 1995, 34, 93-95.

[137] J. D. Hartgerink, J. R. Granja, R. A. Milligan, M. R. Ghadiri, Self-Assembling Peptide Nanotubes, Journal of the American Chemical Society 1996, 118, 43-50.

[138] R. Hourani, C. Zhang, R. van der Weegen, L. Ruiz, C. Li, S. Keten, B. A. Helms, T. Xu, Processable cyclic peptide nanotubes with tunable interiors, Journal of the American Chemical Society 2011, 133, 15296-15299.

[139] D. Mandal, A. Nasrolahi Shirazi, K. Parang, Self-assembly of peptides to nanostructures, Organic \& biomolecular chemistry 2014, 12, 3544-3561.

[140] J. Montenegro, M. R. Ghadiri, J. R. Granja, Ion channel models based on selfassembling cyclic peptide nanotubes, Accounts of chemical research 2013,46 , 2955-2965.

[141] N. Rodriguez-Vazquez, H. Ozores, A. Guerra, E. Gonzalez-Freire, A. Fuertes, M. Panciera, J. Priegue, J. Outeiral, J. Montenegro, R. Garcia-Fandino, M. Amorin, J. Granja, Membrane-Targeted Self-Assembling Cyclic Peptide Nanotubes, CTMC 2015, 14, 2647-2661.

[142] S. Fernandez-Lopez, H. S. Kim, E. C. Choi, M. Delgado, J. R. Granja, A. Khasanov, K. Kraehenbuehl, G. Long, D. A. Weinberger, K. M. Wilcoxen, M. R. Ghadiri, Antibacterial agents based on the cyclic D,L-alpha-peptide architecture, Nature 2001, 412, 452-455.

[143] Y.A. Ovchinnikov, V. T. Ivanov, Conformational states and biological activity of cyclic peptides, Tetrahedron 1975, 31, 2177-2209. 
[144] H. Kessler, Conformation and Biological Activity of Cyclic Peptides, Angew. Chem. Int. Ed. Engl. 1982, 21, 512-523.

[145] S. M. McHugh, J. R. Rogers, H. Yu, Y.-S. Lin, Insights into How Cyclic Peptides Switch Conformations, Journal of chemical theory and computation 2016, 12, 24802488.

[146] Y. Fu, T. Yan, X. Xu, Insight of Transmembrane Processes of Self-Assembling Nanotubes Based on a Cyclic Peptide Using Coarse Grained Molecular Dynamics Simulation, The journal of physical chemistry. B 2017, 121, 9006-9012.

[147] T. Rezai, J. E. Bock, M. V. Zhou, C. Kalyanaraman, R. S. Lokey, M. P. Jacobson, Conformational flexibility, internal hydrogen bonding, and passive membrane permeability: successful in silico prediction of the relative permeabilities of cyclic peptides, Journal of the American Chemical Society 2006, 128, 14073-14080.

[148] S. Thennarasu, A. Tan, R. Penumatchu, C. E. Shelburne, D. L. Heyl, A. Ramamoorthy, Antimicrobial and Membrane Disrupting Activities of a Peptide Derived from the Human Cathelicidin Antimicrobial Peptide LL37, Biophysical journal 2010, 98, 248-257.

[149] R. M. Verly, M. A. Rodrigues, K. R. P. Daghastanli, A. M. L. Denadai, I. M. Cuccovia, C. Bloch, F. Frézard, M. M. Santoro, D. Piló-Veloso, M. P. Bemquerer, Effect of cholesterol on the interaction of the amphibian antimicrobial peptide DD K with liposomes, Peptides 2008, 29, 15-24.

[150] A. Pokorny, L. E. Yandek, A. I. Elegbede, A. Hinderliter, Almeida, Paulo F. F., Temperature and composition dependence of the interaction of delta-lysin with ternary mixtures of sphingomyelin/cholesterol/POPC, Biophysical journal 2006, 91, 2184-2197.

[151] D. Lingwood, K. Simons, Lipid rafts as a membrane-organizing principle, Science (New York, N.Y.) 2010, 327, 46-50.

[152] R. D. Usery, T. A. Enoki, S. P. Wickramasinghe, M. D. Weiner, W.-C. Tsai, M. B. Kim, S. Wang, T. L. Torng, D. G. Ackerman, F. A. Heberle, J. Katsaras, G. W. Feigenson, Line Tension Controls Liquid-Disordered + Liquid-Ordered Domain Size Transition in Lipid Bilayers, Biophysical journal 2017, 112, 1431-1443.

[153] T. D. Lazzara, T.-T. Kliesch, A. Janshoff, C. Steinem, Orthogonal functionalization of nanoporous substrates: control of 3D surface functionality, ACS applied materials 8 interfaces 2011, 3, 1068-1076.

[154] N. Teske, J. Sibold, J. Schumacher, N. K. Teiwes, M. Gleisner, I. Mey, C. Steinem, Continuous Pore-Spanning Lipid Bilayers on Silicon Oxide-Coated Porous Substrates, Langmuir 2017, 33, 14175-14183.

[155] I. Reviakine, A. Brisson, Formation of Supported Phospholipid Bilayers from Unilamellar Vesicles Investigated by Atomic Force Microscopy, Langmuir 2000, 16, 1806-1815.

[156] A. A. Brian, H. M. McConnell, Allogeneic stimulation of cytotoxic T cells by supported planar membranes, Proceedings of the National Academy of Sciences 1984, 81, 6159-6163. 
[157] R. P. Richter, R. Bérat, A. R. Brisson, Formation of solid-supported lipid bilayersan integrated view, Langmuir : the ACS journal of surfaces and colloids 2006, 22, 3497-3505.

[158] C. Hamai, T. Yang, S. Kataoka, P. S. Cremer, S. M. Musser, Effect of average phospholipid curvature on supported bilayer formation on glass by vesicle fusion, Biophysical journal 2006, 90, 1241-1248.

[159] J. Choi, G. Sauer, K. Nielsch, R. B. Wehrspohn, U. Gösele, Hexagonally arranged monodisperse silver nanowires with adjustable diameter and high aspect ratio, Chemistry of materials 2003, 15, 776-779.

[160] S. R. Nicewarner-Pena, R. G. Freeman, B. D. Reiss, L. He, D. J. Pena, I. D. Walton, R. Cromer, C. D. Keating, M. J. Natan, Submicrometer metallic barcodes, Science 2001, 294, 137-141.

[161] G. Riveros, S. Green, A. Cortes, H. Gómez, R. E. Marotti, E. A. Dalchiele, Silver nanowire arrays electrochemically grown into nanoporous anodic alumina templates, Nanotechnology 2006, 17, 561.

[162] W. Lee, S.-J. Park, Porous anodic aluminum oxide: anodization and templated synthesis of functional nanostructures, Chemical Reviews 2014, 114, 7487-7556.

[163] K.-T. Tsai, Y.-R. Huang, M.-Y. Lai, C.-Y. Liu, H.-H. Wang, J.-H. He, Y.-L. Wang, Identical-Length Nanowire Arrays in Anodic Alumina Templates, Journal of Nanoscience and Nanotechnology 2010, 10, 8293-8297.

[164] D. Novo, N. G. Perlmutter, R. H. Hunt, H. M. Shapiro, Accurate flow cytometric membrane potential measurement in bacteria using diethyloxacarbocyanine and a ratiometric technique, Cytometry 1999, 35, 55-63.

[165] G. Calzaferri, D. Brühwiler, S. Glaus, D. Schürch, A. Currao, C. Leiggener, Quantum-Sized Silver, Silver Chloride and Silver Sulfide Clusters, Journal of Imaging Science and Technology 2001, 45, 331-339.

[166] R. Lipowsky, T. Rouhiparkouhi, D. E. Discher, T. R. Weikl, Domain formation in cholesterol-phospholipid membranes exposed to adhesive surfaces or environments, Soft Matter 2013, 9, 8438.

[167] M. J. Sarmento, M. Prieto, F. Fernandes, Reorganization of lipid domain distribution in giant unilamellar vesicles upon immobilization with different membrane tethers, Biochimica et biophysica acta 2012, 1818, 2605-2615.

[168] A. Callan-Jones, B. Sorre, P. Bassereau, Curvature-driven lipid sorting in biomembranes, Cold Spring Harbor perspectives in biology 2011, 3.

[169] R. Dimova, Recent developments in the field of bending rigidity measurements on membranes, Advances in colloid and interface science 2014, 208, 225-234.

[170] D. Nesheva, I. Bineva, Z. Levi, Z. Aneva, T. Merdzhanova, J.C. Pivin, Composition, structure and annealing-induced phase separation in SiOx films produced by thermal evaporation of $\mathrm{SiO}$ in vacuum, Vacuum 2002, 68, 1-9.

[171] N. Tomozeiu. Optoelectronics - Materials and Techniques in Optoelectronics Materials and Techniques (Ed.: P. Predeep), InTech, 2011. 
[172] F. M. Harold, Conservation and transformation of energy by bacterial membranes, Bacteriological Reviews 1972, 36, 172-230.

[173] H. Felle, J. S. Porter, C. L. Slayman, H. R. Kaback, Quantitative measurements of membrane potential in Escherichia coli, Biochemistry 1980, 19, 3585-3590.

[174] H. G. Sahl, M. Kordel, R. Benz, Voltage-dependent depolarization of bacterial membranes and artificial lipid bilayers by the peptide antibiotic nisin, Archives of microbiology 1987, 149, 120-124.

[175] M. Ashrafuzzaman, O. S. Andersen, R. N. McElhaney, The antimicrobial peptide gramicidin S permeabilizes phospholipid bilayer membranes without forming discrete ion channels, Biochimica et biophysica acta 2008, 1778, 2814-2822.

[176] D. Fassler, M. Baezold, Photoprocesses of adsorbed polymethine dyes, Journal of Photochemistry and Photobiology A: Chemistry 1992, 64, 359-368.

[177] S. Pramanik, S. C. Bhattacharya, T. Imae, Fluorescence quenching of 3,7-diamino2,8-dimethyl-5-phenyl phenazinium chloride by $\mathrm{AgCl}$ and $\mathrm{Ag}$ nanoparticles, Journal of Luminescence 2007, 126, 155-159.

[178] A. A. Muenter, Fluorescence lifetimes and sensitization rate constants for dyes adsorbed to silver halide microcrystals, The Journal of Physical Chemistry 1976, 80, 2178-2183.

[179] R. Gill, L. Tian, W. R. C. Somerville, E. C. Le Ru, H. van Amerongen, V. Subramaniam, Silver Nanoparticle Aggregates as Highly Efficient Plasmonic Antennas for Fluorescence Enhancement, J. Phys. Chem. C 2012, 116, 1668716693.

[180] N. A. Shumilova, G. V. Zhutaeva. Encyclopedia of Electrochemistry of the Elements in Encyclopedia of Electrochemistry of the Elements, Marcel Dekker New York, 1978.

[181] E. K. Schmitt, M. Nurnabi, R. J. Bushby, C. Steinem, Electrically insulating poresuspending membranes on highly ordered porous alumina obtained from vesicle spreading, Soft Matter 2008, 4, 250-253. 


\section{$7 \quad$ Appendix}

\subsection{List of figures}

Figure 1.1 Structure of Muraymycin A1 ............................................................. 4

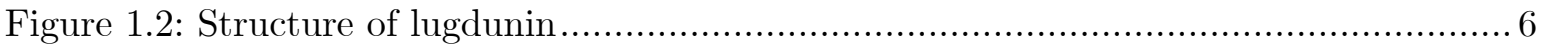

Figure 1.3: Illustration of a permeabilization assay ................................................ 7

Figure 3.1: Structures of the phospholipids POPC, POPE and POPG........................ 12

Figure 3.2: Structures of DOPC, 18:0 SM, and cholesterol...................................... 12

Figure 3.3: Structures of membrane marker dyes used in this thesis......................... 15

Figure 3.4: Structure of the AF488 derivate used to label lipid sidechains ..................... 16

Figure 3.5: Structures of fluorescent dyes used in aqueous solution.............................. 16

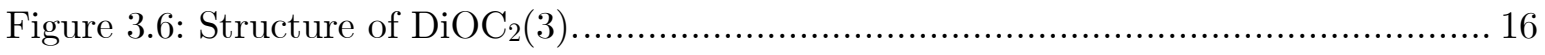

Figure 3.7: Schematic diagram of a fluorescence spectrophotometer. ......................... 17

Figure 3.8 Simplified schematic of a confocal laser scanning microscope...................... 19

Figure 3.9: Schematic view of the conical chamber used for low-volume measurements.... 20

Figure 3.10 Schematic view of an adhered GUV ...................................................... 23

Figure 3.11: Example of a typical measurement and evaluation................................ 24

Figure 3.12: Comparison of the data fits after A: equation ( 1 ) and B: equation ( 2 )...26

Figure 3.13: Schematic of the ACMA assay ................................................... 28

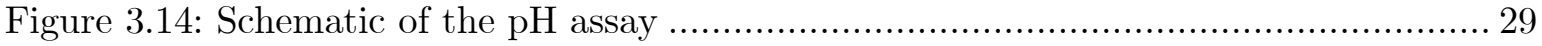

Figure 3.15: Schematic illustration of the permeabilization assay with PSMs on AAO..... 31

Figure 3.16: Schematic depiction of the 2-step anodization process and metal substrate removal. 
Figure 3.17: AAO chip with attached chamber for etching and electro-deposition.

Figure 3.18: Schematic overview of the processing steps performed to obtain an AAO substrate with embedded silver electrodes.

Figure 3.19 AAO chip after gold sputtering

Figure 3.20: Schematic illustration of the orthogonal functionalization of AAO via gasphase silanization with MPTES

Figure 3.21: Schematic illustration of the orthogonal functionalization of AAO with APTES and PEG

Figure 3.22: Schematic illustration of the orthogonal functionalization of AAO via thermal evaporation of $\mathrm{SiO}$.

Figure 3.23: Schematic drawing of the conical chamber with embedded channels for fluidics and electrodes

Figure 3.24: Photographs of the measurement chamber before and after integration at the CLSM.

Figure 4.1: Overview of the AF488 labeled LSC derivates used in this work. 43

Figure 4.2 Orthogonal view of GUVs adhered on an avidin coated silicon wafer in PBS after addition of pyranine to the buffer.

Figure 4.3 A and B: Confocal micrographs of GUVs adhered on avidin coated silica wafers.

Figure 4.4: A and B: Confocal micrographs of adhered GUVs after 40 min of incubation with $0.8 \mathrm{mM}$ AF488-acetate. $\mathrm{C}$ and $\mathrm{D}$ : Relative frequencies of the fill ratios $\mathrm{F} / \mathrm{F}_{0}$.

Figure 4.5: Distribution of fill ratios of adhered GUVs after 40 min of incubation with AF488-acetate for three separate preparations.

Figure 4.6: A and C: Exemplary traces of the normalized fill ratio of individual GUVs over time for AF488-acetate and the $\omega$-hydroxyguanidino-LSC conjugate A1. B and D: Distribution of the maximum rate of dye influx $\mathrm{dF} / \mathrm{dt}$ into individual GUVs

Figure 4.7: Schematic of an adhered GUV and its relation to the PSF of a CLSM.......... 52

Figure 4.8: Apparent partition coefficient for the guanidino-LSC in POPC vesicles at concentrations of $0.4,0.5,0.8,1.6$ and $3.2 \mu \mathrm{M}, \mathrm{n}=25,8,39,9$, and 11 respectively. 53 


\section{Appendix}

Figure 4.9: Membrane intensity divided by the solution intensity plotted against the fill factor for A1-LSC and POPC.

Figure 4.10: Boxplots of the apparent partition coefficients $P_{a p p}$ of different $\omega-$ functionalized AF488-LSC conjugates in GUVs composed of POPC.

Figure 4.11: Linear regression of the fluorescent intensity of solutions of tridecanoic acid AF488-conjugate in PBS at concentrations of $0.2 \mu \mathrm{M}, 0.42 \mu \mathrm{M}$ and $0.8 \mu \mathrm{M}$

Figure 4.12 A: Confocal micrograph of a POPC-GUV incubated with $\omega$-guanidino hexanoic acid AF488 conjugate.. B: Intensity profile of the ROI shown in A

Figure 4.13: A: Confocal micrograph of a POPC-GUV incubated with hexanoic acid AF488 conjugate.. B: Intensity profile and data fit of the ROI shown in A.

Figure 4.14: Fluorescence intensity of membrane bound Atto390 (0.01 mol\%) in consecutive frames of a time series of confocal micrographs.

Figure 4.15 Fluorescence intensity of the GUV membrane and aqueous solution for a FRET-pair of $0.01 \mathrm{~mol} \%$ TexasRed and $0.8 \mu \mathrm{M} \omega$-hydroxyguanidino-LSC.

Figure 4.16 Boxplot illustrating the apparent partition coefficient $P_{a p p}$ of $\omega$ hydroxyguanidin-, guanidine- and amino-LSC conjugates in GUVs composed of POPC, POPC:POPE 7:3, and POPC:POPE 4:6

Figure 4.17 Boxplot of the apparent partition coefficient $P_{a p p}$ of unfunctionalized tridecanoic acid in GUVs composed of POPC and POPC:POPE 7:3.

Figure 4.18: A: Time course of carboxyfluorescein leakage from LUVs composed of POPC and POPC/POPG 50:50, induced by addition of lugdunin or gramicidin S. B: Detailed view of carboxyfluorescein leakage for lugdunin additions only.

Figure 4.19: Time course of ACMA fluorescence intensity showing slow acidification of SUVs due to a negative transmembrane potential after addition of $25 \mathrm{nM}$ valinomycin. Addition of lugdunin (P/L 1:20) halts the acidification, addition of CCCP leads to acidification again.

Figure 4.20: Time course of normalized ACMA fluorescence intensity showing slow acidification of SUVs after addition of lugdunin (P/L 1:50 and 1:100).....

Figure 4.21 Time course of normalized pyranine fluorescence after addition of lugdunin showing proton efflux and subsequent quenching of free pyranine with $4 \mathrm{mM}$ DPX........ 77

Figure 4.22: Comparison of $\mathrm{pH}$ assay fluorescence traces after uncorrected and corrected normalization 
Figure 4.23: Time course of normalized pyranine fluorescence after addition of $25 \mathrm{nM}$ to $5 \mu \mathrm{M}$ Lugdunin (P:L 1:2000 to 1:10) with A,D,G: proton influx from $\mathrm{pH} 6.4$ to 7.4, $\mathrm{B}, \mathrm{E}, \mathrm{H}$ : proton efflux from $\mathrm{pH} 7.4$ to 8.4. Vesicles composed of POPC, containing $0.5 \mathrm{mM}$ pyranine in $\mathrm{NaCl}, \mathrm{KCl}$, or $\mathrm{CsCl}$ buffer. $\mathrm{C}, \mathrm{F}, \mathrm{I}$ : log-log plot of the initial rate $\mathrm{d} I / \mathrm{dt}$, calculated from the normalized fluorescence, vs. the peptide concentration.

Figure 4.24: Time course of normalized pyranine fluorescence after acidification of POPC vesicles that were pre-incubated with lugdunin.

Figure 4.25: Time course of normalized pyranine fluorescence after addition of $20 \mathrm{nM}$ to $5 \mathrm{\mu M}$ Lugdunin (P:L 1:2500 to 1:10) to vesicles composed of POPC or POPG

Figure 4.26 A: Time course of normalized pyranine fluorescence after addition of lugdunin (lugd) or methyl-lugdunin (m-lugd) at P/L ratios of 1:10 to 1:100 B: Structure of mlugdunin. .85

Figure 4.27: Confocal fluorescence micrographs of phase-separated pore-spanning bilayers on porous alumina.

Figure 4.28: Fluorescence micrographs of a phase-separated pore-spanning membrane patch on porous alumina after warming to room temperature.

Figure 4.29: Fluorescence micrographs of a phase-separated pore-spanning membrane patch on porous alumina after spreading at $0{ }^{\circ} \mathrm{C}$.

Figure 4.30: Diffusion coefficients from FRAP measurements for the $l_{d}$-phase (blue) and the $l_{o}$-phase (black) patch on porous alumina at temperatures between $0{ }^{\circ} \mathrm{C}$ and $20{ }^{\circ} \mathrm{C}$.

Figure 4.31: Fluorescence micrographs of a phase-separated pore-spanning membrane patch on a porous $\mathrm{Si}_{4} \mathrm{~N}_{3}$ substrate (pore diameter $0.45 \mu \mathrm{m}$ ) after spreading at $20{ }^{\circ} \mathrm{C}$, showing an apparent conversion of freestanding $l_{d}$-domains to $l_{o}$-phase. Scale bars $10 \mu \mathrm{m}$ 96

Figure 4.32: Fluorescent micrographs of a phase-separated membrane patch on a porous $\mathrm{Si}_{4} \mathrm{~N}_{3}$ substrate, spread at $20{ }^{\circ} \mathrm{C}$ and imaged during heating to $55{ }^{\circ} \mathrm{C}$. .97

Figure 4.33 Fluorescent micrographs of the membrane patch shown in Figure 4.32 at $55{ }^{\circ} \mathrm{C}$ and successively lower temperatures.

Figure 4.34: Scanning electron micrographs of $\mathrm{SiO}$ on AAO.. 102

Figure 4.35: Confocal micrographs of a single AAO substrate after repeated spreading of GUVs.. 103

Figure 4.36 Confocal micrograph of membrane patches on SiO-coated AAO after agitated spreading of GUVs. 103 


\section{Appendix}

Figure 4.37: Cross-sectional SEM micrograph of silver wires embedded in AAO............ 105

Figure 4.38: Photographs of AAO chips during preparation of embedded nanowires.. .... 106

Figure 4.39: Cyclic voltammogram of the nanoelectrode array in PBS.

106

Figure 4.40: Scanning electron micrograph of nanoelectrode array after repeated oxidation and reduction in PBS buffer..

Figure 4.41: Fluorescence response of $\mathrm{DiOC}_{2}(3)$ to a transmembrane potential.............. 109

Figure 4.42: Fluorescence response of pyranine enclosed in porous AAO to a transmembrane potential.

Figure 4.43: Permeabilization of PSMs by $0.5 \mu \mathrm{M}$ melittin.

Figure 4.44: Confocal micrograph of AAO nanoelectrode substrate incubated with pyranine after lysis of PSMs.

\section{$7.2 \quad$ List of tables}

Table 1: Summarized results of the GUV permeabilization experiments on PEG-coated glass.

Table 2: Results for the linear mixed model for $P_{a p p}$ 


\subsection{List of abbreviations}

\begin{tabular}{|c|c|}
\hline $\mathrm{AAO}$ & anodic aluminium oxide \\
\hline ACMA & 9-amino-6-chloro-2-methoxyacridin \\
\hline AF488 & AlexaFluor 488 \\
\hline AFM & atomic force microscopy \\
\hline AMP & antimicrobial peptide \\
\hline APTES & (3-aminopropyl)triethoxysilane \\
\hline ATP & adenosine triphosphate \\
\hline BSA & bovine serum albumin \\
\hline cAMP & cyclic adenosine monophosphate \\
\hline $\mathrm{CCCP}$ & carbonyl cyanide $m$-chlorophenyl hydrazone \\
\hline $\mathrm{CF}$ & carboxyfluorescein \\
\hline cGMP & cyclic guanosine monophosphate \\
\hline CLSM & confocal laser scanning microscopy \\
\hline $\mathrm{CMC}$ & critical micelle concentration \\
\hline DCD & dermcidin \\
\hline DHPE & 1,2-dihexadecanoyl-sn-glycero-3-phosphoethanolamine \\
\hline DMSO & dimethyl sulfoxide \\
\hline DOPC & 1,2-dioleoyl-sn-glycero-3-phosphocholine \\
\hline DPPE & 1,2-bis(diphenylphosphino)ethane \\
\hline DPX & p-xylene-bis-pyridinium bromide \\
\hline DSPE & 1,2-stearoyl-sn-glycero-3-phosphatidylethanolamine \\
\hline FAD & flavin adenine dinucleotide \\
\hline f-PSMs & freestanding membranes \\
\hline FRAP & fluorescence recovery after photobleaching \\
\hline FRET & Förster resonance energy transfer \\
\hline GlcNAc & N-acetylglucosamine \\
\hline GOPTS & 3-glycidyloxypropyltrimethoxysilane \\
\hline
\end{tabular}


7 Appendix

\begin{tabular}{|c|c|}
\hline $\mathrm{GrS}$ & gramicidin $\mathrm{S}$ \\
\hline GUV & giant unilamellar vesicle \\
\hline HEPES & (4-(2-hydroxyethyl)-1-piperazineethanesulfonic acid) \\
\hline HPTS & 8-hydroxypyrene-1,3,6-trisulfonic acid \\
\hline ITO & indium tin oxide \\
\hline$l_{d}$ & liquid-disordered phase \\
\hline$l_{o}$ & liquid ordered phase \\
\hline LSC & lipid side chain \\
\hline LUV & large unilamellar vesicle \\
\hline MPTES & (3-mercaptopropyl)triethoxysilane \\
\hline MurNAc & N-acetylmuramic acid \\
\hline NAD & nicotinamide adenine dinucleotide \\
\hline OWS & optical waveguide spectroscopy \\
\hline$P_{a p p}$ & apparent partition coefficient \\
\hline PBS & phosphate-buffered saline \\
\hline PDMS & polydimethylsiloxane \\
\hline PEG & polyethylene glycol \\
\hline PID controller & proportional-integral-derivative controller \\
\hline PLA & polylactic acid \\
\hline POG & 1-palmitoyl-2-oleoyl-sn-glycero-3-phosphoglycerol \\
\hline POM & polyoxymethylene \\
\hline POPC & 1-palmitoyl-2-oleoyl-sn-glycero-3-phosphocholine \\
\hline POPE & 1-palmitoyl-2-oleoyl-sn-glycero-3-phosphoethanolamine \\
\hline $\mathrm{PP}$ & polypropylene \\
\hline PSF & point-spread function \\
\hline PSM & pore-spanning membrane \\
\hline QCM & quartz crystal microbalance \\
\hline RIfS & reflectometric interference spectroscopy \\
\hline ROI & region of interest \\
\hline SAR & structure-activity relationship \\
\hline
\end{tabular}




$\begin{array}{ll}\text { SM } & \text { sphingomyelin } \\ \text { SPR } & \text { surface plasmon resonance } \\ \text { s-PSM } & \text { solid-supported parts of the membrane } \\ \text { SUV } & \text { small unilamellar vesicle } \\ \text { TLC } & \text { thin-layer chromatography } \\ \text { Trp } & \text { tryptophan } \\ \text { TX-100 } & \text { Triton X-100 } \\ \text { UDP } & \text { undecaprenyl phosphate }\end{array}$




\subsection{List of devices}

BX61 WI, FluoView 1200

LSM 710

FP-6500

NanoDrop2000c

MilliQ Gradient A10

pH-meter Calimatic 766

plasma cleaner Zepto LF PC

Sputtercoater 108 auto

Coating system MED020

Frequency generator 33220A

Ultrasonic bath Sonorex RK255H

Vacuum drying chamber

Thermomixer Compact

LiposoFast-Basic

Hamilton syringes
Olympus, Tokio, Japan

Carl Zeiss, Jena, Germany

JASCO Germany GmbH, Groß-Umstadt, GER

Thermo Scientific (Wilmington, USA)

Merck Millipore, Darmstadt, Germany

Knick, Berlin, Germany

Diener electronic, Ebhausen, GER

Cressington Scientific Instruments, Watford,UK

Bal-TEc, Leica, Wetzlar, Germany

Agilent Technology, Santa Clara, CA, US

Bandelin, Berlin, Germany

Binder, Tuttlingen, Germany

Eppendorf AG, Hamburg, GER

Avestin, Ottawa, CAN

HAMILTON Bonaduz AG, Bonaduz, CHE 


\section{Curriculum vitae}

Personal data

Name Johannes Andreas Schumacher

Date of birth $\quad 02.05 .1984$

Place of birth Nürnberg, Germany

Education

$2014-2020 \quad$ Ph.D. thesis

in the research group Biomolecular Chemistry (Prof. Dr. Claudia

Steinem), Georg-August University, Göttingen

Title of the thesis: Interaction of antimicrobial compounds with lipid

bilayers in established and novel membrane model systems

$2004-2014 \quad$ Diploma in chemistry

Friedrich-Alexander-University Erlangen-Nürnberg

Title of the thesis: Catalytic Surfaces for Liquid Hydrogen Carrier

Conversion with Well Defined Geometry

2003

Allgemeine Hochschulreife Abitur

Albert Schweitzer Gymnasium, Erlangen

Scientific contributions

Peer-reviewed publications

Schilling, N. A., Berscheid, A., Schumacher, J., Saur, J. S., Konnerth, M. C., Wirtz, S. N., Beleña, J. M. B., Zipperer, A., Krismer, B., Peschel, A., Kalbacher, H., Brötz-Oesterhelt, H., Steinem, C., Grond, S. C. (2019) Synthetic lugdunin analogues reveal essential structural motifs for antimicrobial action and proton translocation capability. Angew. Chem. Int. Ed. 58, $9234-9238$.

Teske, N., Sibold, J., Schumacher, J., Teiwes, N. K., Gleisner, M., Mey, I., Steinem, C. (2017) Continuous pore-spanning lipid bilayers on silicon oxide-coated porous substrates. Langmuir 33, 14175-14183.

Schwamborn, M., Schumacher, J., Sibold, J., Teiwes, N.K., Steinem, C. (2017) Monitoring ATPase induced $\mathrm{pH}$ changes in single proteoliposomes with the lipid-coupled fluorophore Oregon Green 488. Analyst 14, 2670-2677. 
Assaud, L., Schumacher, J., Tafel, A., Bochmann, S., Christiansen, S., \& Bachmann, J. (2015). Systematic increase of electrocatalytic turnover at nanoporous platinum surfaces prepared by atomic layer deposition. Journal of Materials Chemistry A, 3(16), 8450-8458.

Roscher, V., Licklederer, M., Schumacher, J., Rios, G. R., Hoffmann, B., Christiansen, S., \& Bachmann, J. (2014). Accurate tuning of ordered nanotubular platinum electrodes by galvanic plating. Dalton Transactions, 43(11), 4345-4350.

\section{Posters}

Johannes Schumacher, Marius Wirth, Christian Ducho, Claudia Steinem: Interaction of the fatty acid moiety of muraymycin antibiotics with lipid bilayers

Annual Meeting of the German Biophysical Society + Satellite Meeting Dynamical Interactions at Biological Membranes, Erlangen 2016

Johannes Schumacher, Ingo Mey, Claudia Steinem: Investigation of membrane permeabilization by antimicrobial peptides - A chip-based model lipid bilayer system

10th European Biophysics Congress, Dresden 2015 


\section{Acknowledgements}

Above all, I want to thank my supervisor Prof. Dr. Claudia Steinem for giving me the opportunity to conduct this project and for her persevering support and patience.

I'd also like to thank Prof. Dr. Michael Meinecke for acting as my second reviewer and for productive discussions in the thesis meetings. Further thanks go to Prof. Dr. Helmut Grubmüller, Dr. Sebastian Kruss, Prof. Dr. Marcus Müller and Prof. Dr. Silvio O. Rizzoli for agreeing to act as members of my examination board.

This work would not have been possible without my cooperation partners at the Universities of Saarland and Tübingen who supplied me with ever more interesting stuff, a big thank you goes to Nadine and Marius.

Thank you to my students Nils, Merve and Fiona for your support with experiments and patience with my ever-changing requirements. Thank you Niko, for helping me conquer the AAO and trying to outdo me with new ideas.

Thank you, Jerry for the vital MATLAB support and all the nice helper scripts that made my life so much easier.

A heartfelt thank you goes to Jutta und Micha for your support in the lab and for supplying me with an abundance of beautiful vesicles and alumina.

To the wonderful secretaries Melanie, Marianne and Dana, thank you for being so great and for having my back in all my organizational issues.

Furthermore, many thanks to Ingo and Anna for proof-reading this thesis. A special thanks also to Darja and Fabian for supplying emergency food in the last critical days of writing.

Thank you to my families, particularly my Anna. I know you've hoped and whished for this day almost as much as me.

To my fellow PhD students of the AK Steinem I want to say thank you for all the wonderful discussions, distractions and delightful do's during these years.

"What I need... is a strong drink and a peer group." - Douglas Adams, Life, the Universe and Everything 
